
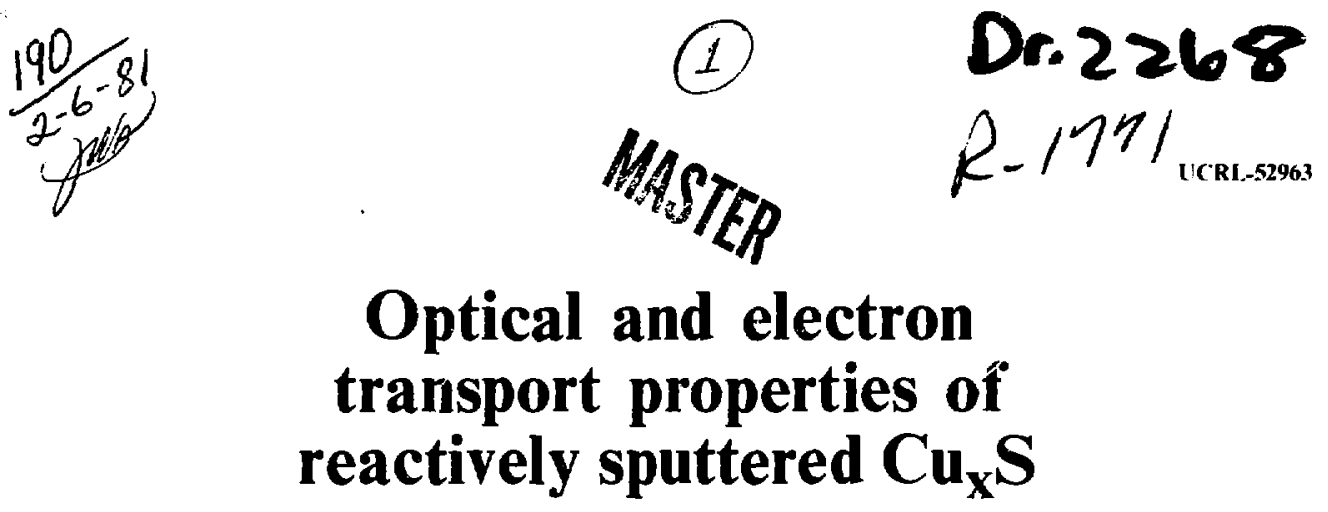

John Yick-Chung Leong

June 30, 1980

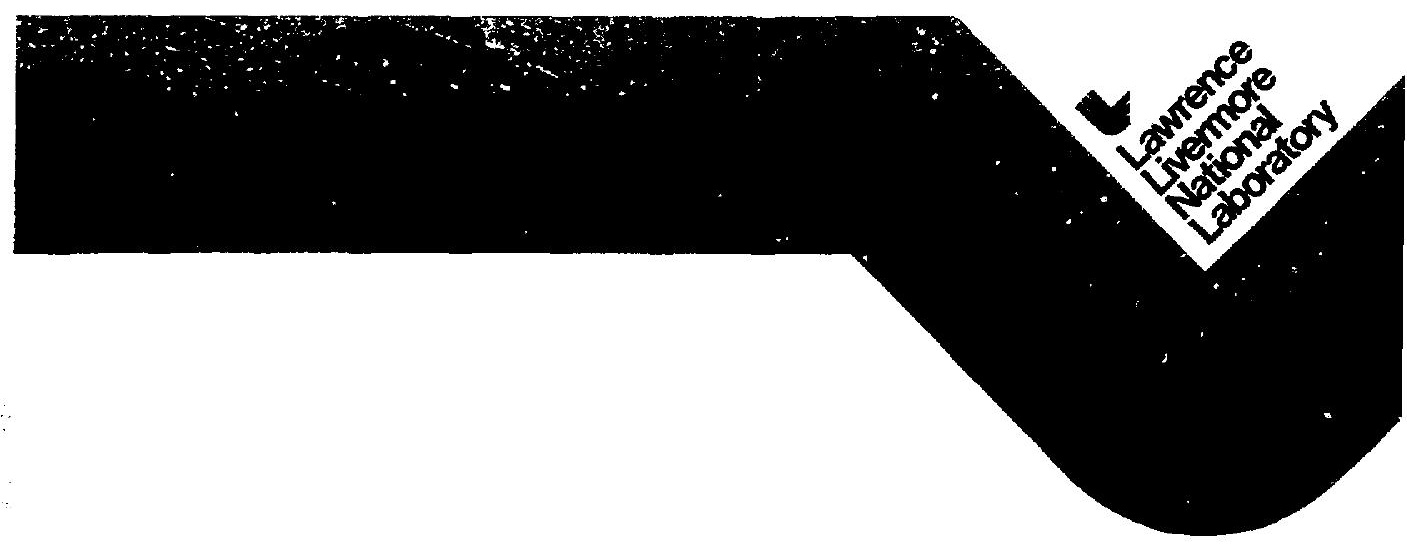


UCRL-52963

Distribution Category UC-38

\title{
Optical and electron transport properties of reactively sputtered $\mathrm{Cu} \mathbf{u}_{\mathbf{x}} S$
}

\author{
John Yick-Chung Leong
}

Manuscript date: June, 1980

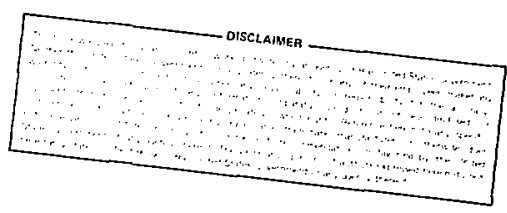

LAWRENCE LIVERMORE LABORATORY University of California - Livermore, California • 94550 


\section{Acknowledgements}

I wish to express my deep appreciation to Dr. Jick H. Yee, my thesis advisor, for his wise counsel and constant moral support through this work.

I am also indebted to Dr. Larry D. Partain for many helpful discussions and for his friendship during the most difficult parts of the thesis research.

To Messrs. Dan Okubo and Bart Brown for pickup up after, covering up for, and putting up with this graduate student, I shall always be grateful.

My aptreciation also goes to the members of the Electron Devires and Electronic Material groups, especially Drs. Guy A. Armantrout and Ralph Kalibjian, for their support and friendship.

Special thanks is due to Mrs. Gail Simpson for $h \cdot r$ cheerful cooperation in typing the numerous revisions of the thesis.

This work was supported by the Lawrence Livermrze Laboratory in conjunction with the Department of Applied Sciences of the University of California--Davis. 
$\underline{\text { Page }}$

List of symbols...................... vi

list of Tables....................... ix

List of Figures. . . . . . . . . . . . . . . . . . x

Abstract ........................ xiii

I. Intraduction ................... 1

II. Background ... . . . . . . . . . . . . . 3

A. The Role of Copper Sulfide in the $\mathrm{Cu} / \mathrm{S} / \mathrm{CdS}$

Solar Gell .................. 3

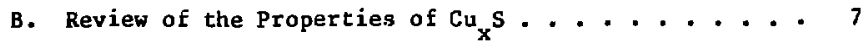

1. Chemical and Structural Properties....... 7

2. Optical Properties............ . 10

3. Electrical Properties. . . . . . . . . 22

III. Theory . . . . . . . . . . . . . . 28

A. Optical Absorption Processes............ 28

1. Instrinsic Absorption. ........... 28

a) Diz t Transitions........... 28

b) Indirect Transitions ............ 32

c) The Urbach Effect............ 33

2. Free Carrier Absorption. . . . . . . . 39

3. Temperature Effects. . . . . . . . . . . . 41

B. Mobility and Scattering Mechanisms... . . . . . 45

1. Lattice Scattering . . . . . . . . . 45

a) Acoustic Phonons and the Deformation Potential 45

b) Piezoelectric Scattering ......... 46

c) Polar Optical Phonon Scattering....... 48 
(cont.)

$\underline{\underline{P a g e}}$

2. Impurity Scattering. . . . . . . . . 50

a) Ionized Impurities . . . . . . . . 50

b) Neutral Impurities............ 54

3. Grain Boundaries and Surface Scattering. . . . . 54

IV. Experimental Methods. . . . . . . . . . . . 58

A. Sample Preparation ................. 58

1. Traditional Deposition Techniques. . . . . . . 58

2. The Sp.ttering Process.............. 60

3. Sample Processing Procedure. . . . . . . . 67

B. Optical Measurements................. 71

1. Instrumentation. ............... 71

2. Data Reduction.............. 75

c. Electrical Measurements. ............... 80

1. Instrumentation. ............ 80

2. The Hall Effect. . . . . . . . . . 84

V. Experimental Results. . . . . . . . . . . . . 92

A. Effects of Sputtering Gas Pressure . . . . . . . . 93

B. Effects of heat Treatment. . . . . . . . . . . 113

C. Temperature Effects on Optical Properties. . . . . 133

VI. Summary and Conclusions................. 141 


\section{Table of Contents \\ (cont.)}

$\underline{\text { Page }}$

Appendices

I. Transmittance and Reflectance of a Multi-layer

Optical Medium. . . . . . . . . . . . . 146

II. Direct Optical Transitions. . . . . . . . . . 153

III. Indirect optical Transitions. . . . . . . . . 159

IV. Ionized Impurities Scattering and the Relaxation

Time Approximation. . . . . . . . . . . . 162

V. Polar Optical Phonon Scattering and the Solution of

Boltzman's Equation by Variational Methods. . . . . . 168

References ...................... 175 


\section{List of Symbols}

A vector potential

${ }^{A_{S, T}}$ cross-sectional area of substrate, target

B magnetic induction

c speed of light

G) capacitance

d sample thickness; lattice parameter

D thickness of cathode dark space

$D_{1}$ diffusion constant of ions

$E_{b}$ energy barrier to bandgap tunneling

$E_{c}$ electron energy at conduction band edge

$E_{d}$ displacement energy of lattice atom

$E_{f}$ Fermi level

$\mathrm{E}_{\mathrm{g}}$ bandgap energy

$E_{g}^{\prime}$ effective bandgap energy

$E_{I}$ activation energy of ionic current

$E_{s}$ sublimation energy

$E_{v}$ electron energy at valence band edge

$E_{1}$ deformation potential

$\bar{E}$ average energy of primary knock-on

$\Delta \mathrm{E} \quad$ Burstein-Moss shift

$\mathscr{E} \quad$ electric field

f fraction of free carriers undergoing specular versus random scattering by a surface

h Planck's constant $(t=h / 2 \pi)$

I current

$J$ current density

$k$ wave number of phonons and of electrons in a crystalline solid

k extinction coefficient

$k_{B}$ Boltzman's constant

$\mathrm{K}$ wave number of photon 


\section{List of Symbols \\ (cont.)}

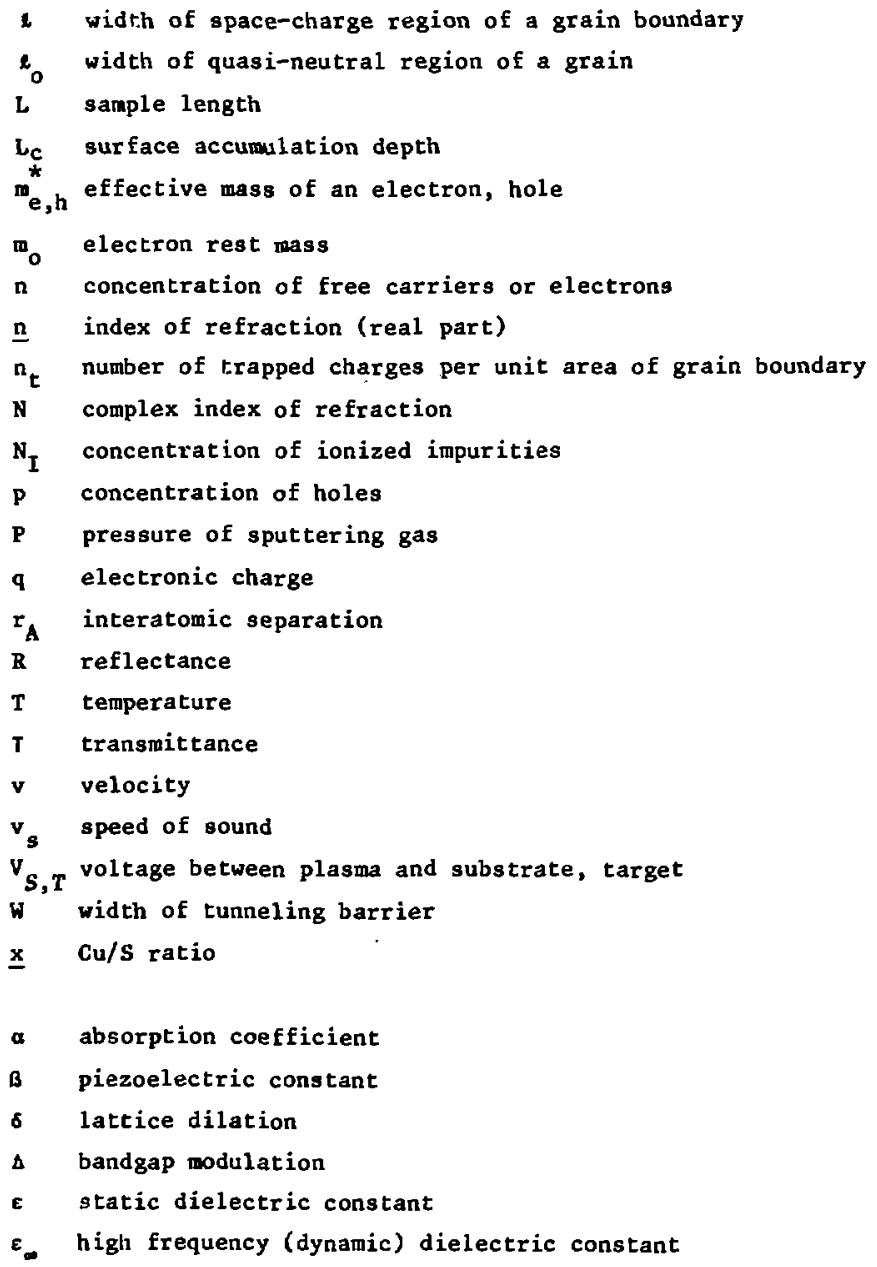




\section{$\frac{\text { List of Symbols }}{\text { (cont.) }}$}

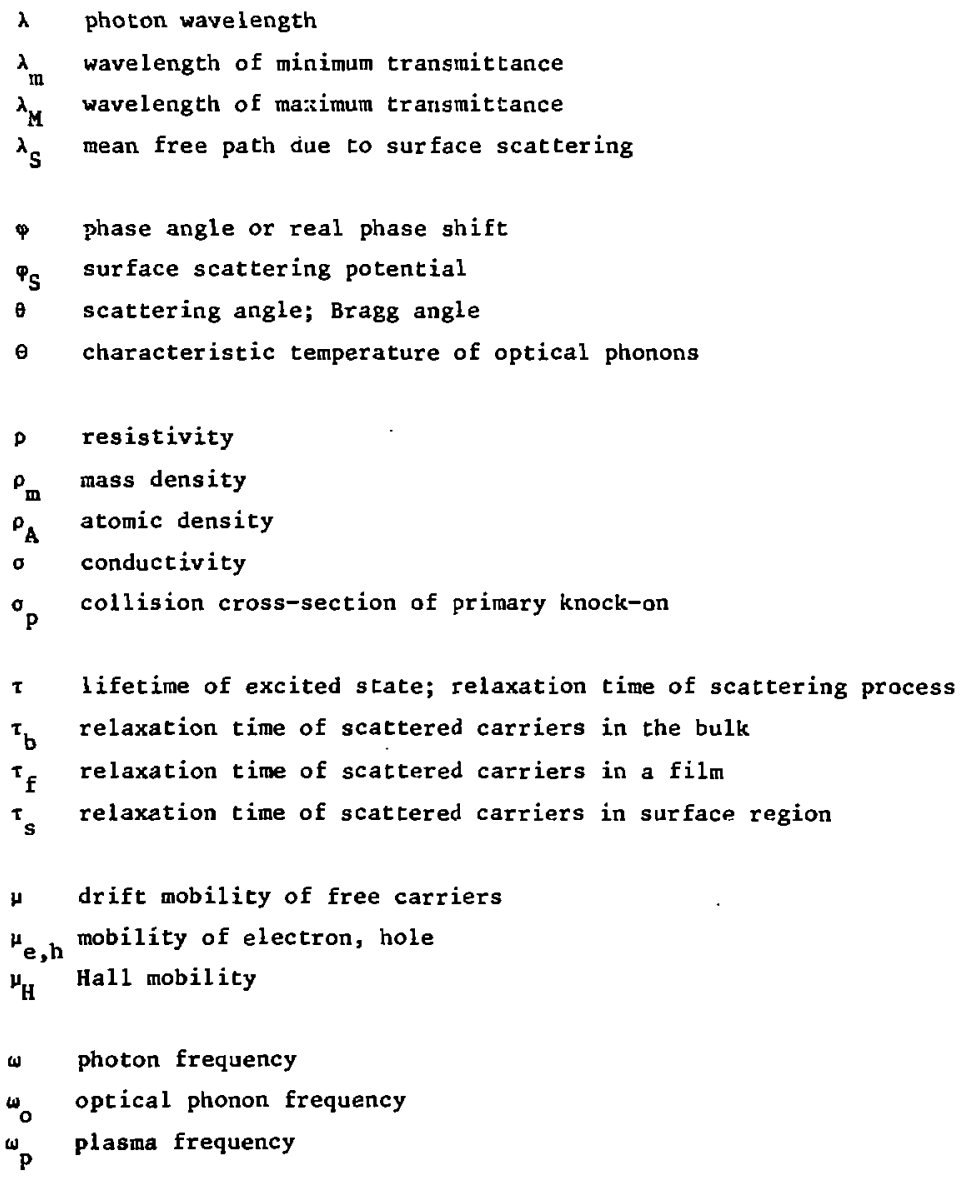




\section{List of Tables}

\section{Page}

1. Bandgaps of $\mathrm{Cu}_{\mathrm{x}} \mathrm{s}$.

21

2. Electrical Properties of $\mathrm{Cu}_{x} \mathrm{~S}$ at Room Temperatures. 27

3. Fermi Integrals. 53 


\section{List of Figures}

$\begin{array}{llr}\text { 1. Band diagram of } \mathrm{Cu}_{\mathrm{X}} \mathrm{S} / \mathrm{C} d \mathrm{~S} \text { solar cell. } & \frac{\text { Page }}{6} \\ \text { 2. Phase diagram from } \mathrm{Cu}_{2.00} \mathrm{~S} \text { to } \mathrm{Cu}_{1.72} \mathrm{~S} & 8\end{array}$

3. Absorption coefficients of polycrystalline $\mathrm{Cu}_{2} \mathrm{~S}$ films. 11

4. Absorption coefficients of thin single crystal Cu $\mathrm{S}$. 13

5. Indices of refraction of thin single crystal Cu $\mathrm{S}$. 14

6. Proposed energy band diagrams for $\mathrm{Cu}_{\mathrm{x}} \mathrm{S}$. 15

7. Emission spectrum of $\mathrm{Cu}_{2} \mathrm{~S}$ at $300^{\circ} \mathrm{K}$. 18

8. Emission spectrum of $\mathrm{Cu}_{2} \mathrm{~S}$ at $77^{\circ} \mathrm{K}$. 19

9a. Band structure of GaAs. 29

$9 \mathrm{~b}$. Band structure of Ge. 29

10. Burstein-Moss shift in degenerate n-type semiconductor. 31

11. Absorption spectra of degenerate Ge. 34

12. Band tailing in degenerate p-type semiconductor. 35

13a. Field induced tunneling. 37

13b. Franz-Keldysh effect. 37

14. Splitting of $3 \mathrm{~s}$ and $3 \mathrm{p}$ states in $\mathrm{si}$. 42

15. Temperature dependent Urbach effect in degenerate p-GaAs. 44

16a. Changes in $\mathrm{E}_{g}$ due to thermal vibrations. 47

16b. Electron scattering by deformation potential. 47

17. Screened polar optical phonon scattering function. 51

18. Barrier model of grain boundaries. 55

19. Principal regions of glow discharge. 61

20. Functional schematic of $\mathrm{rf}$ sputtering system. 64

21. Cu $\mathrm{x}$ samples. 68

22. $\mathrm{H}_{2} /$ Ar heat treatment furnace. 70

23. Function diagram of spectrophotometer. 72

24. Transmission dewar. 73

25. Functional diagram of Hall effect system. 82

26. Hall sample holder and heater. 83 


\section{$\frac{\text { List of Pigures }}{\text { (cont.) }}$}

27a. The Hall effect.

Page

27b. Generalized van der Pauw configuration.

28. Scattering coefficient of polar optical phonon scattering. 89

29. Transmittance and reflectance of high stoichiometry

$\mathrm{Cu}_{\mathrm{x}} \mathrm{s}$ film.

30. Absorption coefficient and index of refraction of high stoichiometry Cu $\mathrm{x}^{\mathrm{s}}$ film.

31. Bandgap analysis of high stoichiometry $\mathrm{Cu}_{x} \mathrm{~S} f i l m$.

32. Urbach effect in high stoichiometry Cu $\mathrm{x}^{\mathrm{S}} \mathrm{film}$.

33. Resistivity and hole concentration of high st ichiometry $\mathrm{Cu}_{\mathrm{x}} \mathrm{s}$ film.

34. Hall mobility of high stoichiometry Cu $\mathrm{x}^{\mathrm{S}}$ film.

35. Transmittance and reflectance of $\mathrm{Cu}_{x} \mathrm{~s}$ films sputtered at different pressures.

36. SEM micrographs of copper nodules.

37. Absorption and index of refraction of $\mathrm{Cu}_{x} \mathrm{~S}$ films sputtered at different pressures.

38. Effects of sputter gas pressure on bandgapg of $\mathrm{Cu}_{\mathrm{x}} \mathrm{s}$ films.

39. Resistivity of $\mathrm{Cu}_{\mathbf{x}} \mathrm{S}$ films sputtered at different $\mathrm{H}_{2}$ S/Ar pressures.

40. Hole concentration of $\mathrm{Cu}_{x} \mathrm{~s}$ films sputtered at different $\mathrm{H}_{2} \mathrm{~S} / \mathrm{Ar}$ pressures.

41. Hall mobility of $\mathrm{Cu}_{\mathrm{x}} \mathrm{s}$ sputtered at different pressures.

42. Effects of air heat treatments on $\mathrm{Cu}_{\mathrm{x}} \mathrm{S}$ transmittance and reflectance.

43. Effects of $\mathrm{H}_{2} / \mathrm{Ar}$ heat treatments on $\mathrm{Cu}_{\mathrm{x}} \mathrm{S}$ transmittance and reflectance.

44. Heat treatment effects on the absorption spectra and indices of refraction in $\mathrm{Cu}_{x} \mathrm{~s}$ films.

45. Effects of heat treatments on band edge. 


\section{$\frac{\text { List of Figures }}{\text { (cont.) }}$}

46. Resistivity of heat treated $\mathrm{Cu}_{x} \mathrm{~S}$ films.

47. Hole concentration of heat treated $\mathrm{Cu}_{x} \mathrm{~s}$ films.

48. Hall mobility of heat treated $\mathrm{Cu}_{x} \mathrm{~S}$ films.

49. Diagnostic x-ray diffraction peaks for djurleite in heat treated films.

50. Diagnostic x-ray diffraction peak for $\mathrm{Cu}_{2} \mathrm{~S}$ in heat treated films.

51. Effects of extended air heat treatment on $\mathrm{Cu}_{\mathrm{x}} \mathrm{S}$ transmittance and reflectance.

52. Phase changes due to extended air heat treatment.

53. Effects of extended $\mathrm{H}_{2}$ /Ar heat treatment on $\mathrm{Cu}_{x} \mathrm{~S}$ transmittance and reflectance.

54. Phase change due to extended $\mathrm{H}_{2} / \mathrm{Ar}$ heat treatment.

55. Transmittance spectra of high stoichiometry $\mathrm{Cu}_{x} \mathrm{~S}$ at $81^{\circ} \mathrm{K}$ and $295^{\circ} \mathrm{K}$.

6. Variations in indirect gap with temperature.

57. Variations in direct gap with temperature.

58. Transmittance spectra of degenerate $\mathrm{Cu}_{\mathrm{x}} \mathrm{S}$ film at $81^{\circ} \mathrm{K}$ and $289^{\circ} \mathrm{K}$.

59. Variations in bandgaps of degenerate $C u_{x} S$ films with temperature.

60. Density and occupancy of states at $289^{\circ} \mathrm{K}$ (a) and $81^{\circ} \mathrm{K}(\mathrm{b})$.

I-1. Light propagation in multi-layer medium. 


\section{Abstract}

Thin films of $\mathrm{Cu}_{x} \mathrm{~s}$ were deposited on glass slides by sputtering $\mathrm{Cu}$ in a reactive $\mathrm{H}_{2} \mathrm{~S} / \mathrm{Ar}$ environment. This structure permitted the measurevent of the optical and electrical properties of thin film $\mathrm{Cu}_{x} \mathrm{~s}$, normally formed on a CdS substrate, with minimal ambiguity, Optical transmittance and reflectance measurements were used to explore the infrared absorption spectra of the material. Analysis of the absorption edge characteristics resulted in the identification of an indirect bandgap at $1.15( \pm .05) \mathrm{eV}$, a direct bandgap at $1.30( \pm .05) \mathrm{eV}$, and an electron effective mass of $1.0( \pm 0.2) \mathrm{m}_{0}$. Electrical data consisting of resistivity and hall effect measurements from liquid nitrugen to room temperatures were analyzed to determine the dominant scattering mechanisms limiting the hole mobility in the material. Ionized impurity scattering was the dominant mechanism at low temperatures ( $\mathrm{T}<100^{\circ} \mathrm{K}$ ) and polar optical phonon scattering was most effective at high temperatures $\left(\mathrm{T}>150^{\circ} \mathrm{K}\right)$. All films were $\mathrm{p}$-type.

The properties of the $\mathrm{Cu}_{x} \mathrm{~S}$ films can be controlled by varying the pressure of the sputtering gas. Highly stoichiometric films can be obtained with total gas pressures of $15 \mu \mathrm{m}-\mathrm{Hg}$ which corresponded to a $\mathrm{H}_{2} \mathrm{~S}$ partial pressures of $1 \mu \mathrm{m}-\mathrm{Hg}$. These films were characterized by a near absence of free carrier absorption, minimum hole concentration $\left(10^{18} \mathrm{~cm}^{-3}\right.$ at $100^{\circ} \mathrm{K}$ and $10^{19} \mathrm{~cm}^{-3}$ at $300^{\circ} \mathrm{K}$ ), and maximum Hall mobility $\left(9 \mathrm{~cm}^{2} / \mathrm{v}-\mathrm{sec}\right.$ at $100^{\circ} \mathrm{K}$ and $5.5 \mathrm{~cm}^{2} / \mathrm{v}-\mathrm{sec}$ at $\left.300^{\circ} \mathrm{K}\right)$. With increasing total pressures $(<30 \mu \mathrm{m}-\mathrm{Hg}$ ) free carrier absorption increased to a level comparable to that of intrinsic absorption. No significant changes in bandgap was observed but the density of states, measured by the effective mass, decreased with increasing pressures. The hole concentration increased by an order of magnitude while the Hall mobility of the holes decreased by a factor of 5 as the pressure increased to $30 \mu \mathrm{m}-\mathrm{Hg}$. 
When the filins were subjected to post deposition heat treatments in air at $180^{\circ} \mathrm{C}$ the effects on the optical and electrical properties were similar to that of increasing the sputtering gas pressures. This led to the conclusion that degeneracy in both cases was due to a common source--increase in $\mathrm{Cu}$ vacancies. In the former case it was due to deposition of the $\mathrm{Cu}_{x} \mathrm{~S}$ in a $\mathrm{Cu}$ deficient environment while in the latter case it was the result of $\mathrm{Cu}$ depletion by oxidation. Conversion from a high to low stoichiometry inixture was confirmed by $x$-ray diffraction. When the same films were heated in $\mathrm{H}_{2} / \mathrm{Ar}$ at the same temperature, the changes in the optical and electrical properties were reversed. These films tend to be more stoichiometric than those exhibiting more degenerace properties but the evidence provided by $x$-ray data was not consistent enough to allow one to make a conclusive statement regarding the effects of $\mathrm{H}_{2}$ /Ar heat treatments. Irprovements in the optical and electrical properties may be due to annealing effects.

Effects of temperature on the band structure of $\mathrm{Cu}_{x} \mathrm{~s}$ were studied by measuring the optical transmittance at temperatures between $80^{\circ} \mathrm{K}$ and $300^{\circ} \mathrm{K}$. Both the direct and indirect gaps decreased with temperature with a temperature coefficient on the order of $10^{-4} \mathrm{eV} /{ }^{\circ} \mathrm{K}$. The temperature coefficient associated with the indirect transitions increases with increasing temperature because the population of phonons, which are required for momentum conservation in indirect transitions, also increases with temperature. 


\section{Introduction}

Copper sulfide ( $\left.\mathrm{Cu}_{x} \mathrm{~S}\right) *$ is an important yet poorly understood solar cell material. It is the active component of the $\mathrm{Cu}_{x} \mathrm{~S} / \mathrm{CdS}$ heterojunction solar cell, currently the most promising low cost alternative to the single crystal silicon cell. But, by comparison to CdS, one of the most studied compound semiconductors, the properties of $\mathrm{Cu}_{\mathrm{x}} \mathrm{s}$ is virtually unknown. A survey of the literature uncovered a collection of incomplete and sometimes contradictory information. Sumparies of past experimental results are presented in Chapter II as background material for this thesis.

The purpose of this thesis research was to study the basic optical and electrical properties of thin film $\mathrm{Cu}_{x} \mathrm{~s}$ which are important for the development of efficient $\mathrm{Cu}_{\mathrm{x}} \mathrm{s} / \mathrm{CdS}$ solar cells and the effects of certain processing procedures on those properties. The $\mathrm{Cu}_{x} \mathrm{~s}$ samples were prepared by a reactive sputtering process which allowed one to study the material as a highly uniform, isolated thin film. The controls afforded by this deposition technique made it possible to reduce some of the ambiguities (e.g., surface roughness, uncertain film thickness, composition gradients) inherent to many of the earlier studies.

Optical transmittance and reflectance measurements were used to determine the absorption coefficient in the visible and infrared regions. The basic theories used to interpret the data are outlined in

*The significance of the variable composition of $\mathrm{Cu}_{x} \mathrm{~s}$ will be clarified in Section II-A. In this work copper sulfide will be referred to as $\mathrm{Cu}_{\mathrm{x}} \mathrm{S}$ with $2 \leq \mathrm{x} \leq 1.8$ unless a particular phase is specified. Results from the 1 iterature will be cited with the composition as claimed by the authors. But the reader should be aware that many of the early works made little or no attempt to verify the phase and composition. Interpretation and evaluation of the results must be made with this qualification in mind. 
Chapter III-A. The derivations of important relationships pertinent to intrinsic absorp $n$ are given in Appendices II and III. The analysis of this data led to a clarification of the magnitude and type of bandgap present in $\mathrm{Cu}_{\mathrm{x}} \mathrm{S}$.

The electrical properties were studied by measuring the resistivity and Hall effect in the thin films at temperatures ranging from $80^{\circ} \mathrm{K}$ to $300^{\circ} \mathrm{K}$. The specific aim of this work was to identify the dominant scattering mechanism controlling the transport of charge carriers. The final interpretation was obtained by comparing the temperature dependence of the data with the functional dependence of a number of plausible scattering mechanisms. These mechanisms are reviewed in Chapter III-B. The derivation of the two most significant mechanisms are detailed in Appendices IV and $V$.

Attempts to modify the optical and electrical properties of the material were made by varying the sputter gas pressure and by subjecting the films to different post deposition heat treatments. The purpose of this portion of the research was to acquire some understanding of how the above processing procedures may be used to optimize the properties of $\mathrm{Cu}_{x} \mathrm{~s}$ for achieving high cell efficiencies. The control on the deposition environment is unique for the reactive sputtering process. The long term goal of the study was to demonstrate the potential of the process for making high efficiency cells. On the other hand, the heat treatments in air and $\mathrm{H}_{2} / \mathrm{Ar}$ have been used by other workers who fabricate $\mathrm{Cu}_{\mathrm{x}} \mathrm{S} / \mathrm{CdS}$ cells by more traditional methods. They found that the heat treatments were often needed to activate a photovoltaic response in the cell. The reason for this effect is still unclear. Possible effects on the sputtered $\mathrm{Cu}_{\mathrm{x}} \mathrm{s}$ were explored. $\mathrm{X}-\mathrm{ray}$ diffraction analysis was used to identify the phases of $\mathrm{Cu}_{x} \mathrm{~S}$ and to monitor the effects of the heat treatments. 
II. Background

A. The Role of $\mathrm{Cu}_{\mathrm{x}} \mathrm{S}$ in the $\mathrm{Cu} \mathrm{s} / \mathrm{CdS}$ Solar Cell

The hist? of the $\mathrm{Cu}_{\mathrm{x}} \mathrm{S} / \mathrm{CdS}$ solar cell began in 1954 , when Reynolds $\mathrm{ft}: \mathrm{l}$ reported the first observations of a photovoltaic effect in s. device. At the time it was thought that the CdS was entirely respousible for the photovoltaic response and that the copper was present only as a contact material. Williams and Bube, [3] in 1960, were the first to point out the importance of copper in the actual photoresponse of the cell. But they explained this contribution of the copper to the long wavelength response (i.e., photon energy less than that of the bandgap of CdS) as one of photoemission from the free metal into the CdS. This theory was disproved in 1962 by Grimeiss and Meming [4] who observed a similar photovoltaic effect in cells in which the free copper had been removed by etching. They proposed an alternate model of a $p-n$ CdS homojunction. The normally n-type CdS is partly converted intc p-type $C d S$ by the diffusion of copper into it. The concentration of copper impuricy is so high that it forms an impurity band. The long wavelength response of the cell was attributed to excitation of electrons from this band into the conduction band.

A heterojunction model of $\mathrm{Cu}_{2} \mathrm{~S} / \mathrm{CdS}$ was first proposed by Cusano $[5]$ in 1963, in the course of discussing his work on Cis $\mathrm{Te} / \mathrm{CdTe}$. He also suggested that the $\mathrm{Cu}_{2} \mathrm{~S}$ may be responsible for some of the long wavelength response, but he concurred with earlier opinions that most of the response was due to the highly doped Cds. $[4,6,7]$

Hi11 and Keramidas, [8] in 1966, were among the first to show that $\mathrm{Cu}_{\mathrm{x}} \mathrm{s}$ was responsible for a significant partion of the photoresponse. In fact, they found that in a newly formed cell, made by dipping a thin film of CdS into an acidified aqueous solution of CuCl, the 
photoresponse was almost entirely due to the $\mathrm{Cu}_{\mathrm{x}} \mathrm{s}$. A short heat treatment was necessary to optimize cell efficiency. It was only then that a significant response due to the CdS appeared, accompanied by a loss in response due to the $\mathrm{Cu}_{x} \mathrm{~s}$.

Selles et al, ${ }^{[9]}$ reporting on an evaporated $\mathrm{Cu}_{2} \mathrm{~s} / \mathrm{CdS}$ cell in 1967, provided further evidence of the importance of the $\mathrm{Cu}_{x} \mathrm{~s}$ in the photogeneration process. The high relative response in the long wavelength region and the cutoff near $1.0 \mu \mathrm{m}$ were explained by a $\mathrm{p}-\mathrm{Cu}_{2} \mathrm{~S} / \mathrm{n}$-CdS heterojunction model with a positive discontinuity in the conduction band (i.e., the electron affinity of the $\mathrm{Cu}_{2} \mathrm{~S}$ was less than that of the (dS). They also noted that the low energy tail of the photoresponse curve had a square root dependence on photon energy which they interpreted as an indication of an indirect gap in $\mathrm{Cu}_{2} \mathrm{~s}$.

In 1968 a group working at the clevite Corporation $[10,11 \mathbf{j}$ presented some of the most convincing evidence of the key role played by the $\mathrm{Cu}_{\mathrm{x}} \mathrm{s}$. Their work consisted of over two years of study on the electrical, optical, and chemical properties of $\mathrm{Cu}_{x} \mathrm{~s} / \mathrm{CdS}$ cells formed by dipping polycrystalline films of $\mathrm{CdS}$ in a CuCl solution. They proposed a heterojunction model which attributed almost all the spectral response to intrinsic absorption in the $\mathrm{Cu}_{\mathrm{x}} \mathrm{s}$. The long wavelength cutoff at $1.2 \mathrm{eV}$ and a rise in the spectral response at $1.8 \mathrm{eV}$ were correlated with similar features in the absorption spectrum of $\mathrm{Cu}_{2} \mathrm{~S}$ and explained by the presence of two band edges at those energies. Long wavelength response increased at the expense of short wavelength response as the thickness of the $\mathrm{Cu}_{\mathrm{x}} \mathrm{s}$ increased. With short wavelength $\mathrm{light}$ absorbed nearer to the surface and farther away from the collector junction this merely demonstrated the importance of transport properties (i.e., diffusion length or mobility) in determining the spectra response of the cells. 
In the following year Nakayama ${ }^{[12]}$ reported similar findings in cells formed by dipping CdS ceramic plates in an aqueous solution of $\mathrm{CuSO}_{4}$. The presence of several phases of $\mathrm{Cu}_{\mathrm{x}} \mathrm{s}$ was confirmed by means of $x$-ray analysis. A shift in the long wavelength cutoff with heat treatment was noted. This was explained by the degeneration of the $\mathrm{Cu}_{2} \mathrm{~S}$ to $\mathrm{Cu}_{1.96}$ with a corresponding increase in the bandgap.

Since then the role of the $\mathrm{Cu}_{\mathrm{x}} \mathrm{s}$ layer as the main source of photogenerated carriers has been universally accepted. Fig. 1 is a simple heterojunction model of the $\mathrm{Cu}_{x} \mathrm{~S} / \mathrm{CdS}$ cell containing most of the essential components. When light of sufficient energy is incident on the $\mathrm{Cu}_{\mathrm{x}} \mathrm{S}$ electron-hole pairs are created. In an efficient cell most of the excess carriers diffuse to the junction where they are collected by the junction field. Some losses occur due to bulk recombination in the $\mathrm{Cu}_{x} \mathrm{~s}$ or the CdS depletion region, diffusion and subsequent recombination at the free surface of the $C u_{x} s$, and recombination at the junction interface. Subsequent studies have concentrated on the nature of the junction and the transport mechanisms across this region. Yet, there are many questions regarding the $\mathrm{Cu}_{x} \mathrm{~s}$ that are unanswered.

Attempts to answer those questions have resulted in a variety of conclusions containing many inconsistencies. Much of the confusion stems from the fact that the copper-sulfide system is very complex and subject to instabilities. Difficulties are encountered not on $1 y$ in the measurement of certain properties, but also in the preparation of the material and the manipulation of independent variables in a controlled manner. 


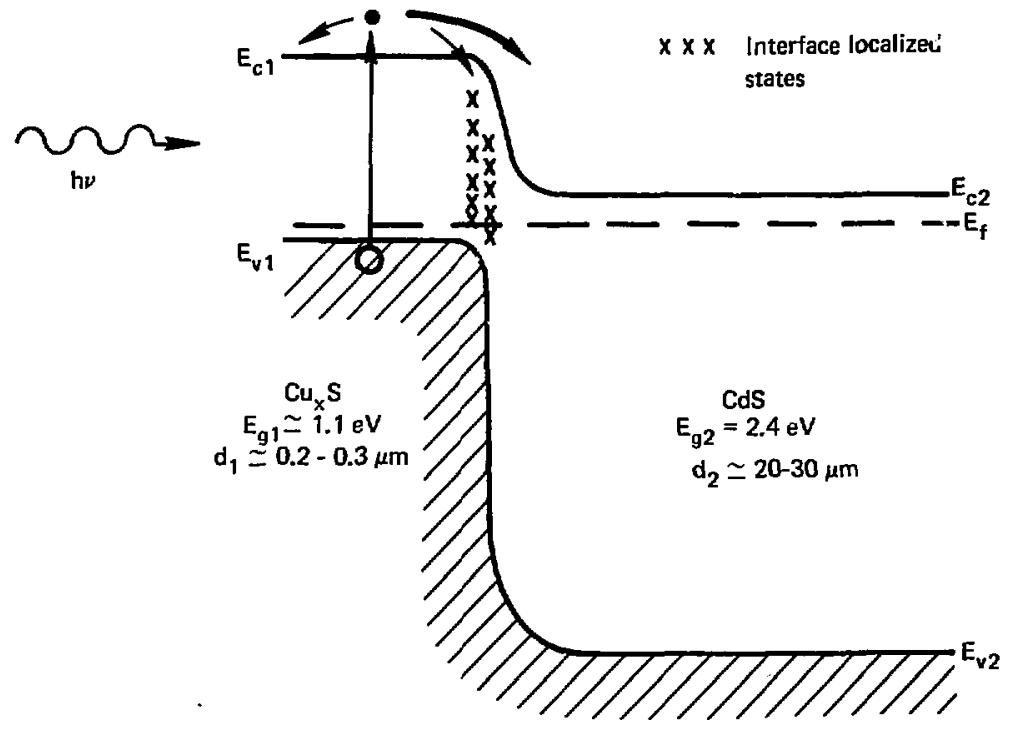

Figure 1. Band diagram of $\mathrm{Cu}_{x} \mathrm{~S} / \mathrm{CdS}$ solar cell. 
B. Review of the Properties of $\mathrm{Cu}_{\mathbf{x}} \mathrm{S}$

1. Chemical and Structural Properties

The Cu-S system contains many phases with dubiously defined boundaries. The difficulty in studying this system is manifested in ongoing controversies concerning fundamental issues such as the existence of certain phases, the basic properties of even the established phases, and the nature of the transformations between them. The phase that is of most interest for solar cell applications is the stoichioLetric chalcocite $\left(\mathrm{Cu}_{2,0} \mathrm{~s}\right)$. Several studies have shown that the most efficient cells are made with this phase. $[13,14,15]$ There are at least two other room temperature phases which deviate from stoichiometry by less than $3 \%$. They are often found with $\mathrm{Cu}_{2.0} \mathrm{~s}$ and can easily be converted from $\mathrm{Cu}_{2.0} \mathrm{~S}$ during the course of cell fabrication. It is for this reason that the material has been referred to as $\mathrm{Cu}_{x} \mathrm{~s}$. It is also the probable cause of the confusion and disagreement among earlier studies. A partial phase diagram of the Cu-s system near stoichiometry is reproduced in Fig. 2 .

$\mathrm{Cu}_{2} \mathrm{~S}$ has an orthorhombic crystal structure at room temperature with lattice constants of $\underline{a}=11.848 \mathrm{~A}, \underline{b}=27.330 \mathrm{~A}$, and $\underline{c}=13.497 \mathrm{~A}$. [16] The extraordinarily large unit cell of $\mathrm{Cu}_{2} \mathrm{~S}$ draws attention to another difficulty in studying the material. The method of modeling a band structure with Bloch functions, successfully applied to silicon and germanium, cannot be used on $\mathrm{Cu}_{2} \mathrm{~S}$ which has 96 molecules or 288 atoms in its unit cell. [17] Lacking a unifying theoretical model, it is very difficult to resolve inconsistent experimental data. At $103^{\circ} \mathrm{C}$, $\mathrm{Cu}_{2} \mathrm{~S}$ transforms to a smaller hexagonal structure with $\underline{a}=3.961 \wedge, \underline{c}=6.722 \mathrm{A.}[16]$

The two most recognized non-stoichiometric plases are djurleite $\left(\mathrm{Cu}_{\mathrm{x}} \mathrm{S}, 1.96<\underline{x}<1.93\right)^{[18,19]}$ and digenite $\left(\mathrm{Cu}_{1.8} \mathrm{~s}\right) .[20]$ The former bas a pseudo-orthorhombic structure with $\underline{a}=15.71 \mathrm{~A}, \underline{\mathrm{b}}=13.56 \mathrm{~A}$, $\underline{c}=26.95 \mathrm{~A}{ }^{[21]}$ The latter is pseudo-cubic with $\underline{a}=5.56 \mathrm{~A}$. [18] 


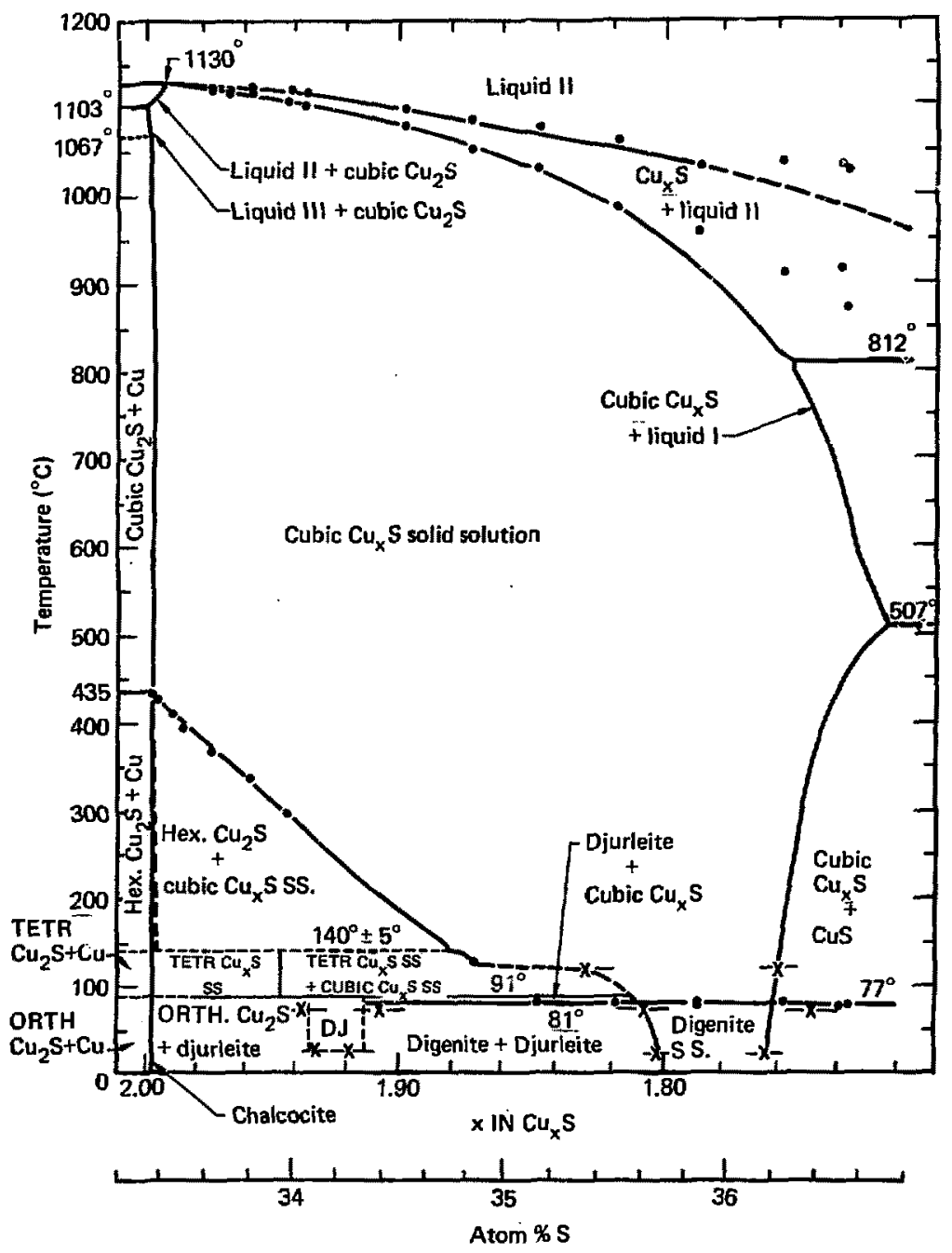

Figure 2. Partial phase diagram of $\mathrm{Cu}_{x} \mathrm{~S}[19]$. 
A fourth room temperature phase, $\mathrm{Cu}_{1.9} \mathrm{~S}$, has been reported by some workers. $[22,23]$ It is hexagonal with $a=11.355 \dot{A}, \underline{c}=13.506 \mathrm{~A}$. [21]

As early as 1941, Buerger ${ }^{[24]}$ noted that chalcocite can degenerate to digenite when it was heated in air. More recently, Cook ${ }^{[19]}$ reported that chalcocite can degenerate to djurleite when exposed to air for several months or heated in $\mathrm{Ar}$ at $90^{\circ} \mathrm{C}$ for several days. In both cases it was believed that oxidation of the copper had taken place. In the latter case it was due to residual oxygen. Conversion to digenite occurred less readily. Heating in air to a temperature as high as $435^{\circ} \mathrm{C}$ was needed to activate the reaction. The explanation offered by cook for the different rates of the reacions was that chalcocite and djurleite have similar superstructures of hexagonal close packed sulfur acoms while the digenite had a cubic close packed structure for its sulfur atoms. The conversion of chalcocite to djurleite involves the formation of copper vacancies which have a low formation energy of $0.36 \mathrm{ev} .[19]$ On the other hand, the conversion to digenite requires the rearrangement of the very stable sulfur sublattice. Sulfur vacancies needed to initiate restructuring are not present in sufficient numbers at temperatures below $400^{\circ} \mathrm{C}$. The formation energy is $2.7 \mathrm{eV} .{ }^{[19]}$ on the basis of these arguments, conversion of chalcocite to $\mathrm{Cu}_{1.9} \mathrm{~S}$, if it is a true phase, should be relatively easy.

The composition of a $\mathrm{Cu}_{\mathrm{x}} \mathrm{s}$ sample is not a well controlied parameter and one can be led to wrong conclusions about other properties if this fact is ignored. To emphasize this point, the observations of Sorokin

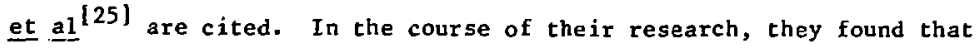
$x$-ray analysis of over 500 samples of what was belie red to be $\mathrm{Cu}_{2} \mathrm{~S}$ indicated that the actual composition and phase were very sensitive functions of sample preparation. Recently, it has been demonstrated by Armantrout ef al that the stoichiometry of $\mathrm{Cu}_{x} \mathrm{~s}$ films can be controlled in reactive sputtering by choosing appropriate sputtering parameters. [26] This control was utilized to study the effects of changing stoichiometry on the transport and optical properties of $\mathrm{Cu}_{x} \mathrm{~s}$. 


\section{Optical Properties}

It is generally agreed that $\mathrm{Cu}_{2} \mathrm{~S}$ has a very high absorbance for $v$ isible 1 ight with an absorption coefficient ( $\alpha$ ) between $10^{4}$ and $10^{5} \mathrm{~cm}^{-1}$. [11,23,27-34] The absorption spectra of some polycrystalline $\mathrm{Cu}_{\mathrm{x}} \mathrm{s}$ films are presented in Fig. 3. The high absorbance makes it possible to fabricate thin film cells with less than 0.5 um of $\mathrm{Cu}_{2} \mathrm{~S}$. The lower efficiencies in degraded cells has been attributed to the presence of non-stoichiometric phases with lower absorbance. $[13,14,15]$ The region of the absorption spectrum that is the subject of much debate is the absorption edge from which one can derive the width of the bandgap $\left(E_{g}\right)$ and determine whether the gap is direct or indirect. A clear understanding of the bandgap is needed to determine the ultimate efficiency of the $\mathrm{Cu}_{x} \mathrm{~s} / \mathrm{CdS}$ solar cell. Loferski[35] has shown that the magnitude of $\mathrm{E}_{\mathrm{g}}$ alone can affect cell efficiency. The type of gap is important because a direct gap is accompanied by a high a while an indirect gap can result in a longer excess charge carrier lifetime.

The earliest optical measurements on $\mathrm{Cu}_{x} \mathrm{~S}$ were made by Eisenmann [27] in 1952, using polycrystalline Films (50 to $300 \mathrm{~nm}$ thick) prepared by evaporating $\mathrm{Cu}_{2} \mathrm{~S}$, onto quartz substrates. He calculated a of films containing different mixtures of $\mathrm{Cu}_{2} \mathrm{~S}, \mathrm{Cu}_{1.85}$, and CuS from their transmittance $(T)$ and reflectance $(R)$. He found that as Cis increased relative to the other phases, free carrier absorption increased at the expense of interband transitions. Although no $\mathrm{E}_{\mathrm{g}}$ was specified, the minimum in a was estimated at $1.0 \mathrm{eV}$ and increased in energy as the Cus concentration increased.

The first attempt to define the band edge of $\mathrm{Cu}_{x} \mathrm{~S}$ was carried out by Marshall and Mitra ${ }^{[28]}$ in 1965. Their samples were polycrystailine, thick $(\sim 80 \mu \mathrm{m})$ films prepared by vapor deposition. The material had the orthorhombic structure of $\mathrm{Cu}_{2} \mathrm{~S}$. They found an indirect gap of $1.21 \mathrm{eV}$ at $300^{\circ} \mathrm{K}$ which increased to $1.26 \mathrm{eV}$ at $80^{\circ} \mathrm{K}$. The urusually low absorption coefficient has not been supported by subsequent studies. 


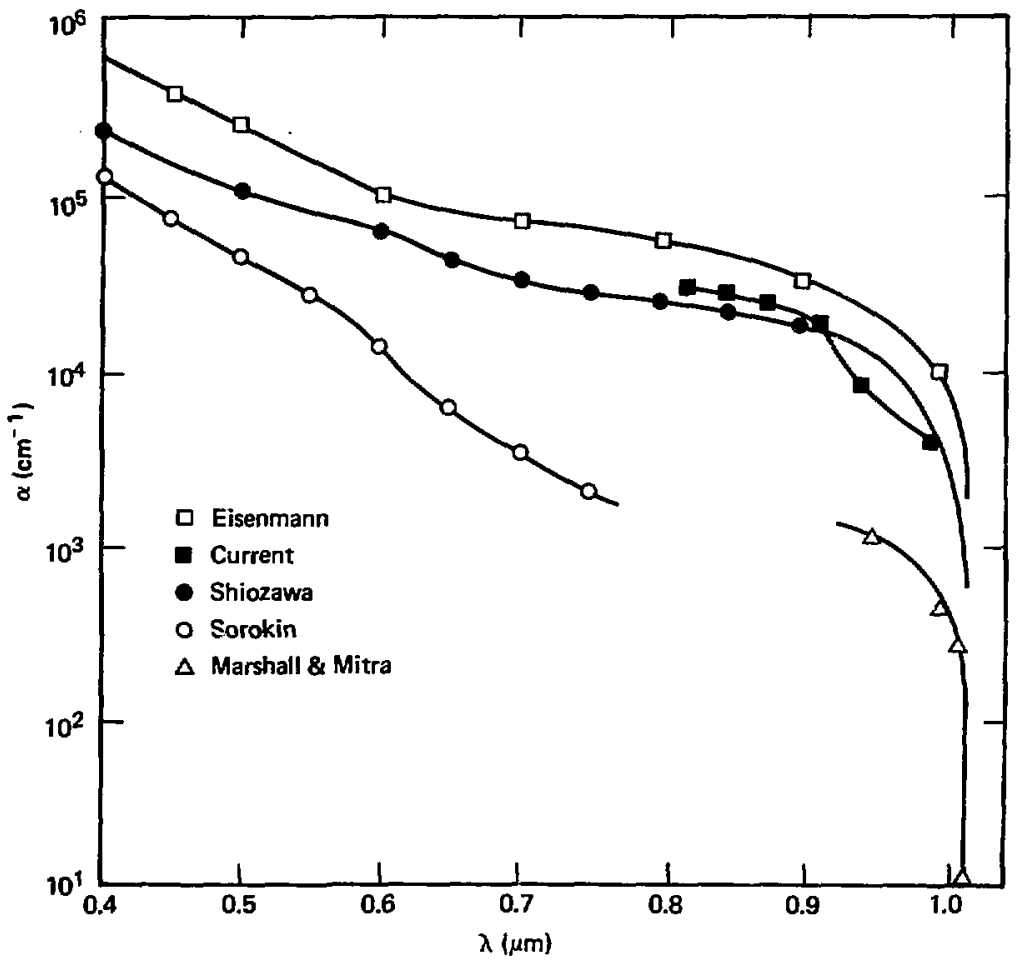

Figure 3. Absorption coefficient or polycrystalline thin film $\mathrm{Cu}_{2} \mathrm{~S}$ [11]. 
The rost often cited work on the optical properties of $\mathrm{Cu}_{x} \mathrm{~S}$ is a series of studies by Mulder. $[23,29,30]$ His samples were thin single crystals $(0.2$ to $2 \mu \mathrm{m})$ converted from $\mathrm{CdS}$ in aqueous solutions of a cuprous salt. $\mathrm{Cu}_{2} \mathrm{~s}$ was formed first, then djurleite, $\mathrm{Cu}_{1.9} \mathrm{~s}$, and digenite were obtained successively by controlled oxidation. Phase changes were monitored by noting color changes in polarized light and checked by chemical analysis of the copper loss. The absorption spectra of the samples were obtained with incident light polarized perpendicular to and parallel with the c-axis of the original CdS. The absorption coefficients and indices of refraction of these samples are reproduced in Fig 4. and Fig. 5, respectively. He found that with the loss of copper, 1) free carrier absorption increased proportionately; 2) the effective bandgap $\left(\dot{E}_{g}^{\prime}\right)$ increased; and 3 ) the gap changed from an indirect gap for $\mathrm{Cu}_{2} \mathrm{~S}$ and djurleite to a direct gap for $\mathrm{Cu}_{1.9} \mathrm{~S}$. He proposed a single rigid energy band model, shown in Fig. 6a, to explain the behavior of all three phases. [29] The observed changes in optical properties with phase change were explained by the lowering of the Fermi level $\left(\mathrm{E}_{\mathrm{f}}\right)$ into the valence band as copper vacancies increased. This is similar to the Burstein-Moss shift in InSb. $[36,37]$ in this model both $\mathrm{Cu}_{2} \mathrm{~S}$ and djurleite have indirect gaps because $E_{Z}$ is above the edge of the direct valence band. At the threshold transition energy the transition is an indirect one. The minimum gap, co:responding to $\mathrm{Cu}_{2} \mathrm{~s}$, is estimated to be at $1.2 \mathrm{eV}$. [30] The $E_{g}^{\prime}$ of djurlaite is larger because copper vacancies acting as acceptors have depopulated the top of the valence band. As the concentration of copper vacancies increases for $\mathrm{Cu}_{1.9} \mathrm{~S}$, Mulder asserts that the valence band becomes so depopulated that the dominant transitions are direct ones. $\mathrm{Cu}_{1.9} \mathrm{~S}$ appears to have a large direct gap near $1.8 \mathrm{e}^{\mathrm{t}}$ where $\alpha$ starts to increase sharply.

Studies by others have added little to current understanding. Some supported, while others contradicted Mulder's model. Sorokin et al ${ }^{[31]}$ measured the photoconductivity, reflectance, and transmittance spectra 


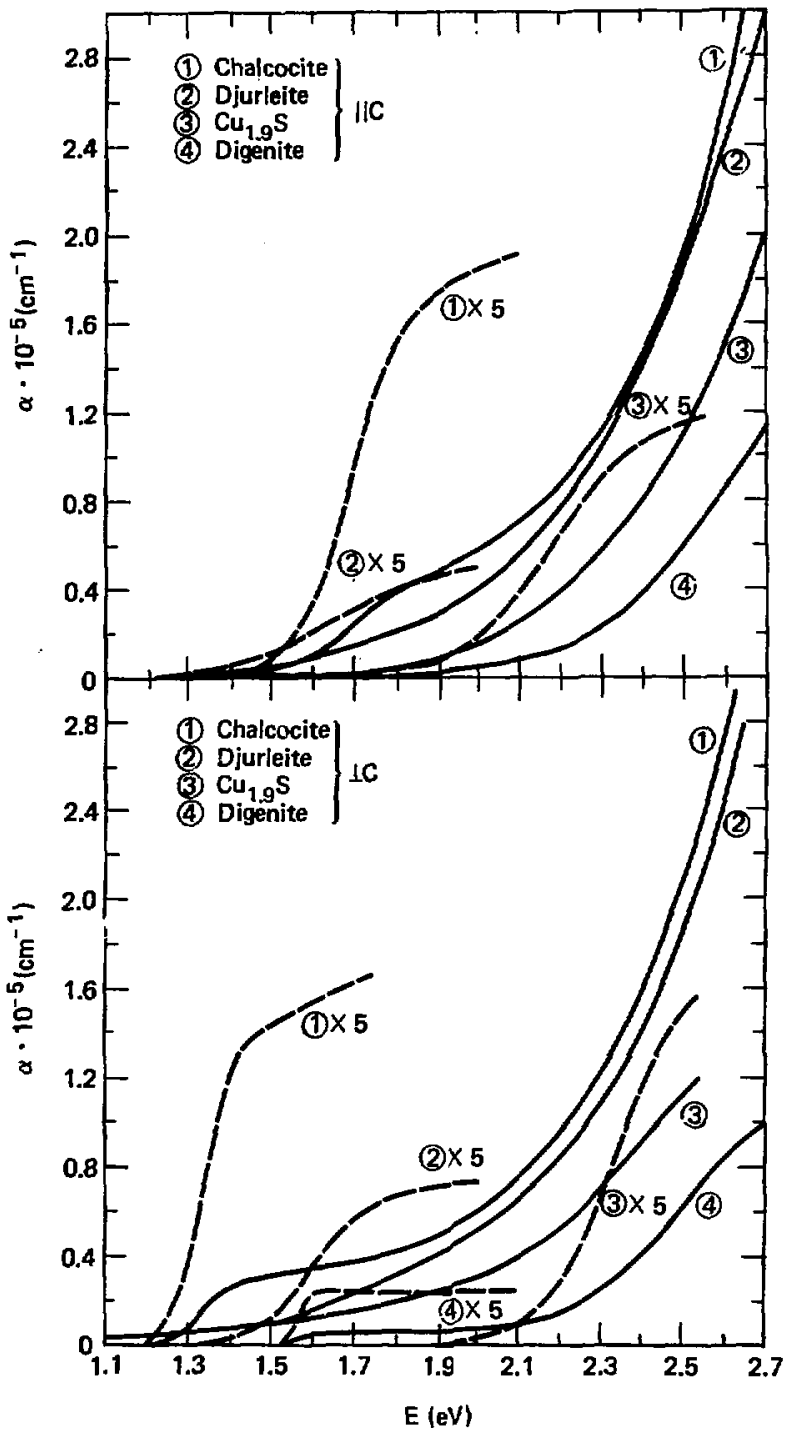

Figure 4. Absorption coefficients of single crystal $\mathrm{Cu}_{\mathrm{x}} \mathrm{S}$ [23]. 

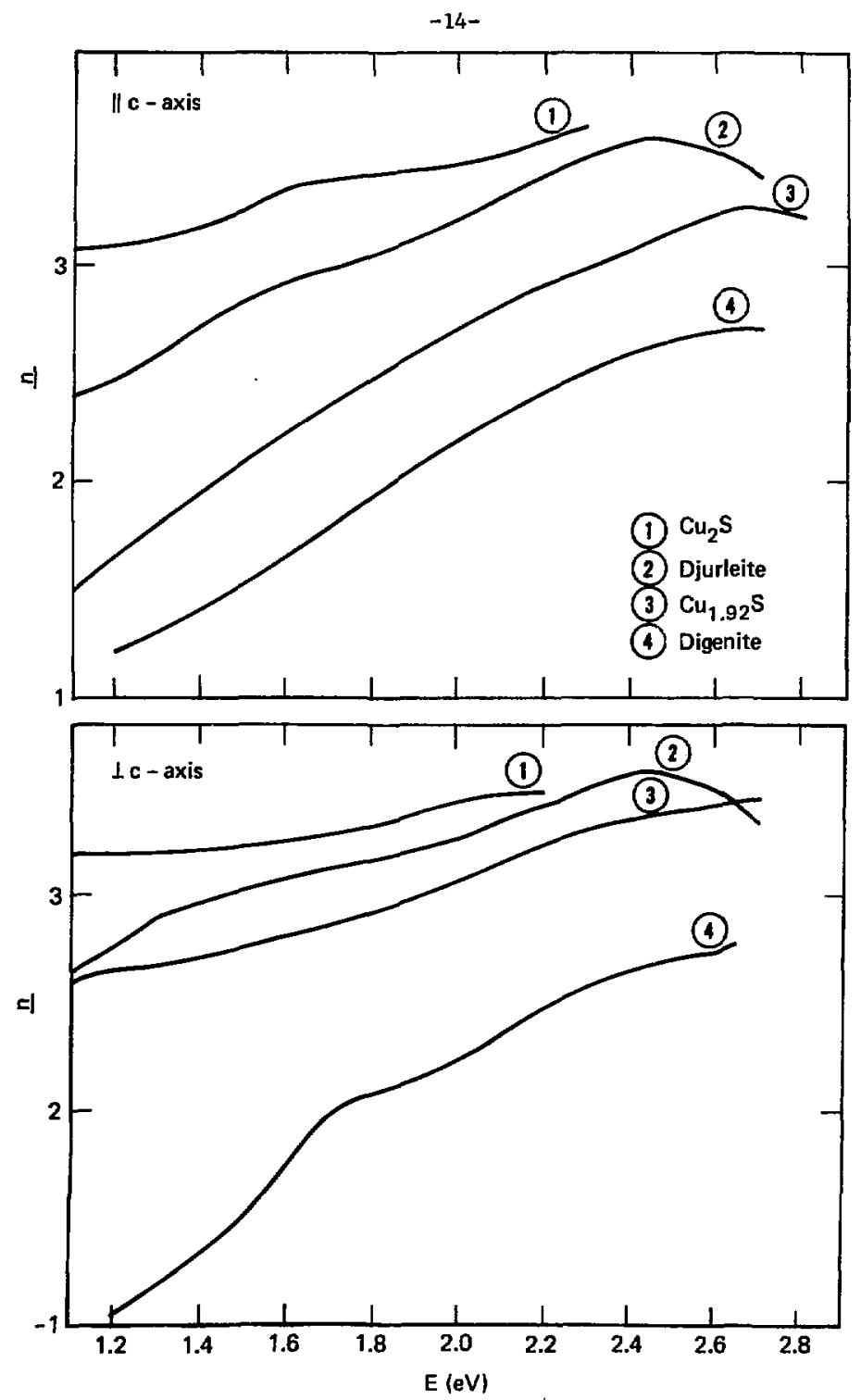

Figure 5. Indices of refraction of single crystal $\mathrm{Cu}_{x} \mathrm{~S}[23]$. 


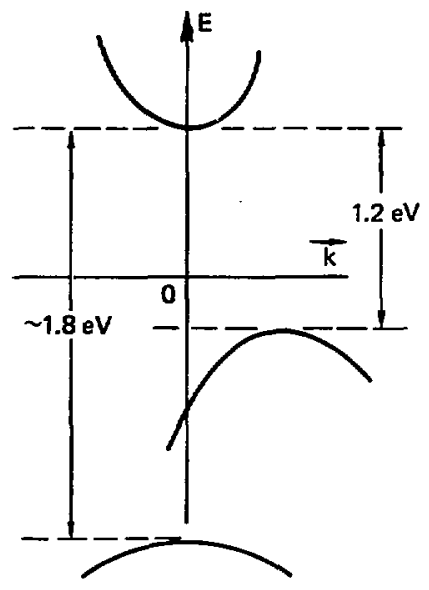

(a)

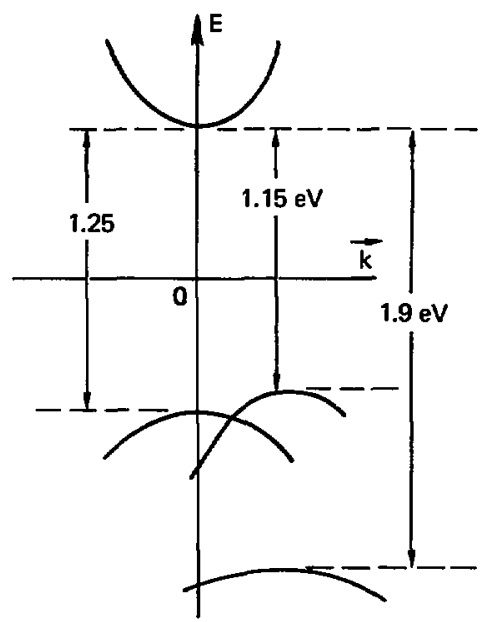

(b)

Mulder [29]

Yee [43]

Figure 6. Proposed energy band diagram for $\mathrm{Cu}_{x} \mathrm{~S}$. 
of evaporated $\mathrm{Cu}_{2} \mathrm{~S}$ and found a bandgap of $1.84 \mathrm{eV}$. They did not specify the type of gap, nor did they give any details regarding phase verification. Nakayama, [32] found indirect gaps of $1.0 \mathrm{eV}$ and $2.3 \mathrm{eV}$ for chalcocite and digenite, respectively. Shiozawa et al ${ }^{[11]}$ came closest to agreeing with Mulder. From their analysis of evaporated $\mathrm{Cu}_{2} \mathrm{~S}$ films on Pyrex substrates and $\mathrm{Cu}_{2} \mathrm{~S}$ layers converted from $\mathrm{CdS}$ by a wet dip process they obtained an indirect gap at $1.2 \mathrm{eV}$ and a direct gap at $1.8 \mathrm{eV}$. Additional support was reported by Ramoin et al ${ }^{\text {[33] }}$ in their study of flash evaporated $\mathrm{Cu}_{2} \mathrm{~S}$ films. They found an indirect gap at $1.05 \mathrm{eV}$ and a direct gap at $1.7 \mathrm{eV}$ in evaporated $\mathrm{Cu}_{2} \mathrm{~s}$ films.

More recently, Couve et al ${ }^{[34]}$ observed a direct gap that increased from $1.85 \mathrm{eV}$ to $2.16 \mathrm{eV}$ as $x$ increased from 1.89 to 1.94 , contrary to Mulder's model. The Cu-s ratio, $\underline{x}$, was determined by an electrochemical method, but the actual crystalline phase could not be ascertained by $x$-ray analysis, probably because the film samples were too thin $(\sim 0.6 \mu \mathrm{m})$. An indirect gap was not detected because tre data near $1.2 \mathrm{eV}$ was obscured by interference effects.

A second point of contention with Mulder's model was brought up by Shewchun et al ${ }^{[38]}$ in their discussion of cathodoluminescence in $\mathrm{Cu}_{2} \mathrm{~S}$. They examined bulk $\mathrm{Cu}_{2} \mathrm{~S}$ as well as thin films $(\sim 0.2$ to $2.0 \mathrm{~mm})$ prepared by sulfurization of Cu. X-ray analysis was used for phase verification. They obtained a luminescence peak in $\mathrm{Cu}_{2} \mathrm{~S}$ at $1.28 \mathrm{eV}$ which is very close to Mulder's indirect gap. But they argued that the narrow half-width $(0.07 \mathrm{eV})$ of the peak is more characteristic of a direct gap. This draws attention to an apparent inconsistency in Mulder's model. If there is an indirect gap at $1.2 \mathrm{eV}$ tne absorption coefficient due to indirect transitions should be much less than the observed $10^{4} \mathrm{~cm}^{-1}$. In silicon, with an indirect bandgap of $1.1 \mathrm{eV}$,

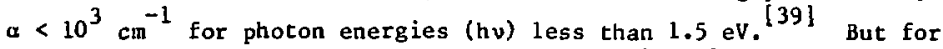
$\mathrm{Cu}_{2} \mathrm{~S}$, in spice of a larger bandgap, $\alpha=5 \times 10^{4} \mathrm{~cm}^{-1}$ at hu $=1.5 \mathrm{eV}$. Such a large $a$ is not expected unless hv $>1.8 \mathrm{eV}$ for which direct 
transitions dominate. Yet the extraordinarily large $\alpha$ has been observed in various forms of $\mathrm{Cu}_{2} \mathrm{~S}$ and is a major reason for the success of $\mathrm{Cu}_{x} \mathrm{~S}$ in making thin film cells.

Cathodoluminescence studies were later performed on $\mathrm{Cu}_{x} \mathrm{~S}$ films prepared by other methods, including the clevite "wet dip" process and the Philips "dry" process, [40] by Loferski et al. ${ }^{[41,42]}$ They fround a peak at $1.23 \mathrm{eV}$ with a half-width of $0.1 \mathrm{eV}$ for $\mathrm{Cu}_{2} \mathrm{~S}$ prepared by the Clevite and Philips processes. They also observed a much less intense peak at $1.36 \mathrm{eV}$ in a material identified by $x$-ray analys is to be tetragonal $\mathrm{Cu}_{1.96} \mathrm{~S}$ which is very close in composition to djurleite. This seens to support earlier findings that the bandgap, either real or effective, increases with decreasing $\underline{x}$. The reduced intensity is explained by the increase in acceptor concentration resulting from $\mathrm{Cu}$ vacancies which provide alternative recombination paths that are nonradiative. This argument can be extended to explain the apparent absence of luminescence in samples with $\underline{x}<1.96$.

Recently, Yee ${ }^{[43]}$ proposed a band model, shown in Fig. 6b, which was able to resolve some of the apparent inconsistencies. Instead of two valence bands he pointed out that spin-orbit interaction and the effect of the orthorhombic crystal field in $\mathrm{Cu}_{2} \mathrm{~S}$ would produce at least three valence band maxima. The results are an indirect gap at $1.15 \mathrm{eV}$, a direct gap at $1.25 \mathrm{eV}$, and a second indirect gap at $1.9 \mathrm{eV}$. The two close lying smaller gaps explains the observed direct and indirect absorption characteristics near the band edge. Schewchun's and Loferski's cathodoluminescence data were used to support rather than refute the indirect minimum gap. A theoretical fit of the data at $77^{\circ} \mathrm{K}$ and $300^{\circ} \mathrm{K}$, shown in Figs. 7 and 8 , indicated that the skewed characteristic of the luminescence peaks was more consistent with an indirect minimum gap. 


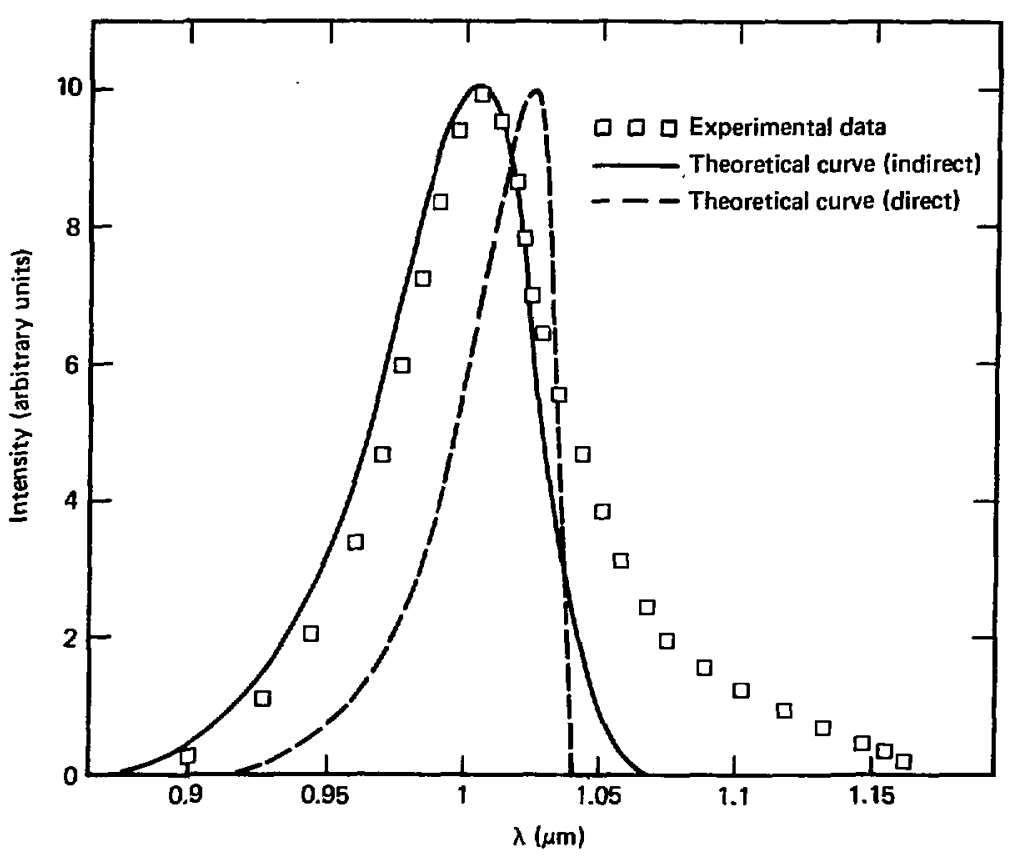

Figure 7. Emission spectrum of $\mathrm{Cu}_{2} \mathrm{~S}$ at $300^{\circ} \mathrm{K}$ [43]: 


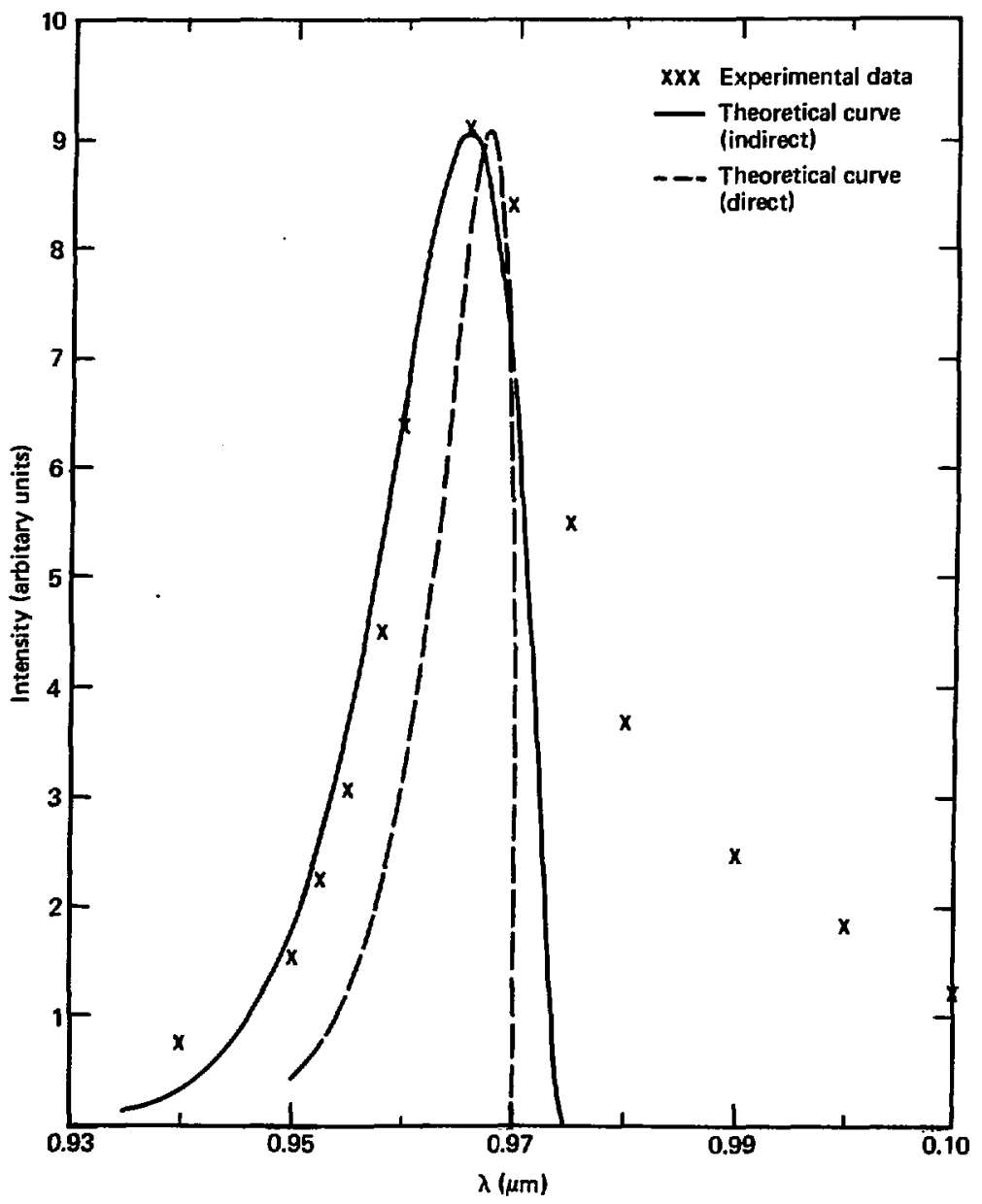

Figure 8. Emission spectrum of $\mathrm{Cu}_{2} \mathrm{~S}$ at $77^{\circ} \mathrm{K}$ [43]. 
The present work will attempt to clarify the puzzling points brought up by previous researchers. In particular, Mulder's and Yee's models will be tested by studying films with different stoichiometry at various temperatures. If in fact a rigid band nodel can be applied to the various nearly stoichiometric phases, changing composition and temperature should allow one to probe various regions of the band struciure. At low temperatures the dissimilarity between the different phases should be resuced, thus revealing the unperturbed band structure.

A sumrary of the reported data presented in this section is presented in Table 1 . 
TABLE 1. Band gaps of $\mathrm{Cu}_{\mathrm{x}} \mathrm{S}$.

\begin{tabular}{|c|c|c|}
\hline Experimenters & Sample forms & $\mathrm{E}_{\mathrm{g}}$ \\
\hline [27] Eisenmann ('52) & polycrystalline thin films $(\sim 0.1 \mu \mathrm{m})$ & $\approx 1.0 \mathrm{eV}$ \\
\hline [28] Marshall and Mitra ('65) & polycrystalline thick films $(\sim 80 \mu \mathrm{m})$ & $1.21 \mathrm{eV}$ (indirect) \\
\hline [31] Sorokin et al ('65) & polycrystalline films & $1.93 \mathrm{eV}$ \\
\hline [32] Nakayama ('68) & polycrystalline films & $\begin{array}{ll}1.0 & \mathrm{eV} \text { (indirect) } \\
2.3 & \mathrm{eV} \text { (direct) }\end{array}$ \\
\hline [11] Shiozawa et al ('69) & $\begin{array}{l}\text { polycrystalline films and single } \\
\text { crystal layers converted fron CdS }\end{array}$ & $\begin{array}{l}1.2 \text { eV (indirect) } \\
1.8 \text { eV (direct) }\end{array}$ \\
\hline [33] Ramoin et al ('69) & Polycrystalline films & $\begin{array}{l}1.05 \mathrm{eV} \text { (indirect) } \\
1.7 \mathrm{eV} \text { (direct) }\end{array}$ \\
\hline$[29,30]$ iqulder ('73) & $\begin{array}{l}\text { single crystal thin films }(\sim 1 \mu \mathrm{m}) \\
\text { converted from CdS }\end{array}$ & $\begin{array}{l}1.2 \mathrm{eV} \text { (indirect) } \\
>1.5 \mathrm{eV} \text { (direct) }\end{array}$ \\
\hline [34] Couve et al ('73) & polycrystalline thin films $(\sim 0.6 \mu \mathrm{m})$ & $2.0 \mathrm{eV}$ (direct) \\
\hline [38] Shewchun et al ('75) & bulk and thin films & $1.28 \mathrm{eV}$ (direct) \\
\hline [41] Loferski et al $(76,79)$ & bulk and thin films & $1.23 \mathrm{eV}$ (direct) \\
\hline
\end{tabular}




\section{Electrical Properties}

Excess charge carriers generated in $\mathrm{Cu}_{\mathrm{x}} \mathrm{S}$ by the absorption of photons of appropriate energies must be transported efficiently through the $\mathrm{Cu}_{\mathbf{x}} \mathrm{S}$ in order to contribute to the photovoltaic effect in the $\mathrm{Cu}_{\mathrm{x}} \mathrm{s} / \mathrm{CdS}$ cell. The transport process is controlled by one or more scattering mechanisms and can be characterized by the mobility ( $\mu$ ) of the carriers.

Some of the earliest attempts to charactarize $\mathrm{Cu}_{\mathrm{x}} \mathrm{s}$ in terms of its transport properties were carried out by Hirahara. $[44,45,46]$ He measured the conductivity $(\sigma)$ and Hall effect in bulk $\mathrm{Cu}_{x} \mathrm{~s}$ for $-20^{\circ} \mathrm{C} \leq \mathrm{T} \leq 250^{\circ} \mathrm{C}$. For stoichiometric samples and $\mathrm{T}<110^{\circ} \mathrm{C}$, $\sigma$ was on the order of $10^{5} \mathrm{a}^{-1}-\mathrm{cm}^{-1}$ and hole mobility $\left(\mu_{h}\right)$ on the order of $10 \mathrm{~cm}^{2} / \mathrm{v}-\mathrm{sec}$. Both decreased with decreasing temperature. Conductivity increased when excess sulfur was introduced, but mobility decreased as a result of the increased scattering by higher concentrations of impurity centers. A sharp drop of about two orders of magnitude in o occurred $i_{n}$ samples with 0.17 excess copper. Mobility was again lower, but by only half as much as that of an equivalent excess of sulfur. This was probably due to a smaller scattering cross-section associated with $\mathrm{Cu}$ impurities. The hole concentration in $\mathrm{Cu}_{2} \mathrm{~S}$ was about $10^{20} \mathrm{~cm}^{-3}$. An order or magnitude increase or decrease was associated with a $0.1 \%$ copper deficit or excess, respectively. Impurity levels of $0.05 \mathrm{eV}$ to $0.10 \mathrm{eV}$ were identified. [44]

An interesting phenomenon was observed as the temperature was increased above $110^{\circ} \mathrm{C}$. An additional current component due to the $\mathrm{d} r$ ift motion of copper ions was detected, accompanied by a sharp drop of about 2 orders of magnitude in electronic current. The ionic current had an activation energy $\left(E_{I}\right)$ of $0.4 \mathrm{eV}$. As a result of the ion motion the crystal lattice field became distorted and conduction electrons and holes experienced increased scattering. 
Miyantani and Suzuki $[47,48]$ also observed the ionic current in $\mathrm{Cu}_{2} \mathrm{~S}$. They derived an empirical expression for the ionic conductivity

$$
\sigma_{I}=\sigma_{0} \exp \left(-E_{I} / k T\right)
$$

where $\sigma_{0}=2.4 \Omega^{-1}-\mathrm{cm}^{-1}$, and $E_{I}=0.30 \mathrm{eV}$.

More recently, Okamoto and Kawai ${ }^{[49]}$ extended the study of ionic conduction to nonstoichiometric $\mathrm{Cu}_{x} \mathrm{~s}$. They found that the ionic conductivity is almost independent of composition with $\sigma_{0}=140 \Omega^{-1}-\mathrm{cm}^{-1}$ and $E_{I}=0.24 \mathrm{eV}$. By using transient techniques, they estimated the equilibrium concentration of Cu vacancies $\mathrm{N}_{\mathrm{I}}=10^{19} \mathrm{~cm}^{-3}$, ionic mobilicy $\mu_{I}=0.2 \mathrm{~cm}^{2} / \mathrm{v}-\mathrm{sec}$, and hole mobility $\mu_{\mathrm{h}}=0.3 \mathrm{~cm}^{2} / \mathrm{v}-\mathrm{sec} .{ }^{12}$

Yokota [50] modeled the mixed conduction process and obtained fits to the data of Miyantani and Suzuki. The time constant associated with the drift is

$$
\tau_{I}=\frac{\sigma_{h}+\sigma_{1}}{\sigma_{h}}\left(\frac{L}{\pi}\right)^{2} \frac{l}{D_{I}}
$$

where $\sigma_{h}$ and $\sigma_{I}$ are the conductivities due to the holes and ions, respectively,

$L$ is the length of the sample,

and $D_{I}$ is the diffusion constant of the ions.

The steady state voltage across the sample is given by

$$
v=\frac{k_{b} T}{q} \text { in }\left(\frac{1+\xi}{1-\xi}\right)
$$

where $\xi=q I L / 2 k_{B} \mathrm{To}_{h}<1$,

$q$ is the electronic charge,

$k_{B}$ is Boltzman's constant,

and $I$ is the current. 
Samples with very low $\mathfrak{u}_{\mathrm{h}}$ may be subjected to large, sustained drifts. Ionic drifts have not been detected for $\mathrm{T}<100^{\circ} \mathrm{C}$ in normal conductivity measurements on $\mathrm{Cu}_{x} \mathrm{~S}$, but they may contribute significant error in Hall voltage measurements.

Hall effect in single crystal $\mathrm{Cu}_{2} \mathrm{~S}$ was measured by Abdullaev et al. [51] At room temperature they found $\sigma=170 \Omega^{-1}-\mathrm{cm}^{-1}$, $\mathrm{p}=7 \times 10^{19} \mathrm{~cm}^{-3}$, and $\mu_{\mathrm{h}}=25 \mathrm{~cm}^{2} / \mathrm{v}-\mathrm{sec}$. They also observed $\mathrm{a} \mathrm{T}-3 / 2$ dependence for $\mu_{h}$ from 20 to $250^{\circ} \mathrm{C}$ which is characteristic of acoustic phonon scattering. They identified an acceptor level at $0.064 \mathrm{eV}$ and a bandgap of $1.8 \mathrm{eV}$. An effective hole mass $\mathrm{m}_{\mathrm{h}}^{\mathrm{x}}=0.58 \mathrm{~m}_{0}$ was computed.

Similar measurements were performed by Bougnot et al ${ }^{[52]}$ over a lower temperature range $\left(77^{\circ} \mathrm{K}<\mathrm{I}<300^{\circ} \mathrm{K}\right)$. The room temperature conductivity ranged from 19 to $1400 \Omega^{-1}-\mathrm{cm}^{-1}$ as $\underline{x}$ varied from 2.00 to 1.85. Hall data was not available for highly stoichiometric samples. For $1.85<\underline{x}<1.94$, $\omega_{h}$ remained nearly constant at $5 \mathrm{~cm}^{2} / v$-sec while $p$ increased from $4 \times 10^{20} \mathrm{~cm}^{-3}$ to $1.8 \times 10^{21} \mathrm{~cm}^{-3}$ as $x$ decreased.

Early studies on thin films $(50-300 \mathrm{~nm})$ of $\mathrm{Cu}_{x} \mathrm{~S}$ were carried out by Eisenmann. [27] $\mathrm{Cu}_{2} \mathrm{~s}$ films were prepared by complete evaporation of a small amount of bulk $\mathrm{Cu}_{2} \mathrm{~S}$. Non-stoichiometric films were obtained by exposing the $\mathrm{Cu}_{2} \mathrm{~S}$ films to $\mathrm{S}$ vapor at elevated temperatures for various lengths of time. The resistivity $(\rho)$ of $\mathrm{Cu}_{2} \mathrm{~S}$ films was $10^{2} \Omega-\mathrm{cm}$. The fact that bulk $\mathrm{Cu}_{2} \mathrm{~S}$ had the same $\rho$ was the main criteria for believing such films to have stoichiometric compositions. The films were too thin for $x$-ray analysis. Such films were hard to get and keep because they were readily oxidized by : th temperature air. A plot of $p$ vs $1 / T$ for $-250^{\circ} \mathrm{C}<\mathrm{T}<23^{\circ} \mathrm{C}$ revealed an activation energy of $0.6 \mathrm{eV}$. A sharp drop in $\rho$ to $10^{-2}$ s $\mathrm{cm}$ as $x$ decreased from 2.0 to 1.8 was reported. 
Sorokin and Paredenko [53] measured the Hall effect in thin film and polycrystalline bulk $\mathrm{Cu}_{2} \mathrm{~s}$. The phases of bulk samples were identified by $x$-ray diffraction analysis. They reported that orthorhombic $\mathrm{Cu}_{2} \mathrm{~s}$, containing an excess of free $\mathrm{Cu}$, had $\sigma=500 \mathrm{a}^{-1}-\mathrm{cm}^{-1}, p=6.16$ $\times{ }^{2}{ }^{18} \mathrm{~cm}^{-3}$, and $\mu_{h}=520 \mathrm{~cm}^{2} / \mathrm{v}-\mathrm{sec}$ which is much higher than that reported by other studies. Temperature dependence of $\sigma$ and $\mu_{h}$ identified an activation energy of $0.09 \mathrm{eV}$ and acoustic phonon scattering as the dominant scattering mechanism for $23^{\circ} \mathrm{C}<\mathrm{T}<300^{\circ} \mathrm{C}$. When excess $S$ was introduced $\sigma$ and $\mu_{h}$ increased while $p$ decreased slightly (by less than 1 order of magnitude). Hexagonal $\mathrm{Cu}_{2} \mathrm{~S}$ was obtained by quenching from $650^{\circ} \mathrm{C}$. Room temperature $o, \mu_{h}$, and $p$ were reduced dramatically. For cu-excess samples $\delta=4.2 \Omega^{-1}-\mathrm{cm}^{-1}, p=4.1$ $\times 10^{17} \mathrm{~cm}^{-3}$, and $\mu_{h}=64 \mathrm{~cm}^{2} / \mathrm{v}-\mathrm{sec}$. Further reductions by nearly an order of magnitude occurred when excess $s$ was added. Thin films $(0.1$ to $5 \mu$ ) were, formed by sublimating the bulk samples. Phases in the films were not identified. It was believed that since $\mathrm{Cu}_{2} \mathrm{~S}$ was a "nondissociable" compound the composition of the thin films was the same'as that of the bulk sources. The conductivity of all films was about $4 \Omega^{-1}-\mathrm{cm}^{-1}$. Films prepared from $\mathrm{Cu}$ rich or thorhombic sources were reported to have $\mu_{h}=40 \mathrm{~cm}^{2} / \mathrm{v}-\mathrm{sec}$ and $\mathrm{p}=5 \times 10^{18} \mathrm{~cm}^{-3}$. The deviations from bulk properties, particularly the reduced $\mu_{h}$, were attributed to grain boundary effects which were more dominant in the thin films. Mobility increased linearly from about $1 \mathrm{~cm}^{2} / v-s e c$ for $0.1 \mu \mathrm{m}$ films to a saturated value of about $5 \mathrm{~cm}^{2} / \mathrm{v}$-sec. The temperature dependence of the carrier mobility in bulk and thin film $\mathrm{Cu}_{2} \mathrm{~S}$ was claimed to have the charactertistic $\mathrm{T}^{-3 / 2}$ of acoustic phonon scattering for $23^{\circ} \mathrm{C}<\mathrm{T}<200^{\circ} \mathrm{C}$.

The study was extended by Sorokin et al ${ }^{[54]}$ to include single crystal nonstoichiometric $\mathrm{Cu}_{x} \mathrm{~s}$. During the sample preparation they identified three distinct crystal structures for $1.73<\underline{x}<2.00$ : a small hexagonal structure $(\underline{a}=3.895 \mathrm{~A}, \underline{c}=6.690 \mathrm{~A})$ for the virtually stoichiometric composition $\underline{x}>1.98$; a much larger orthorhombic structure $(\underline{a}=11.8 \mathrm{~A}, \underline{b}=27.1 \mathrm{~A}, \underline{c}=13.5 \mathrm{~A})$ for $1.97>\underline{x}>1.82$; and a facecentered cubic structure $(\underline{a}=5.5 \AA$ ) for $\underline{x}<1.82$. The cubic phase is 
digenite but the other two had been identified by others as the high and low temperature phases of chalcocite. They also found that, contrary to a past assumption, [11] the final composition after crystallization rended to be more sulfur $r$ ich than the starting composition. They measured formation energy of $\mathrm{Cu}$ vacancies in $\mathrm{Cu}_{\mathrm{x}} \mathrm{S}$ of about $0.25 \mathrm{eV}$ which is much lower than the $1 \mathrm{eV}$ vacancy formation energy of typical semiconductors. They derived empirical relationships between $\underline{x}$ and the various electrical parameters:

$$
\sigma=\sigma_{0}+\underline{A x}
$$

with $\quad \sigma_{0}=0.5 \Omega^{-1}-\mathrm{cm}^{-1}, A=10^{4} \Omega^{-1}-\mathrm{cm}^{-1}$

$$
p=p_{0}+B \underline{x}^{3}
$$

with $\quad \mathrm{p}_{\mathrm{o}}=10^{17} \mathrm{~cm}^{-3}, \mathrm{~B}=2 \times 10^{23} \mathrm{~cm}^{-3}$

$$
h_{h}=\frac{C}{D+\underline{x}^{2}}
$$

with $\quad C=0.2 \mathrm{~cm}^{2} / \mathrm{v}-: \mathrm{eec}, \mathrm{D}=2 \times 10^{-4}$

For stoichiometric samples $\sigma=60 \Omega^{-1}-\mathrm{cm}^{-1}, \mathrm{p}=10^{18} \mathrm{~cm}^{-3}$, and $\mu_{h}=300$ to $600 \mathrm{~cm}^{2} / \mathrm{v}$-sec. To explain the dramatic drop in $\mu_{h}$ in the degenerate orthorhombic and cubic structures, they postulated the presence of two types of holes in $\mathrm{Cu}_{x} \mathrm{~s}$. A low effective mass hole is the dominant carrier for $\underline{x}>1.98$. They attributed this type of hole to the loss of an electron from the cuvalent $\mathrm{Cu}-\mathrm{s}$ bond or from the $\mathrm{s}$ ion. A much heavier hole which is due to $C u$ vacancies is the dominant carrier for $x<1.95$.

A summary of reported electrical data is presented in Table 2. It demonstrates again the general state of confusion that exists with regard to the properties of $\mathrm{Cu}_{\mathbf{x}} \mathrm{S}$. 
TABLE 2. Electrical properties of $\mathrm{Cu}_{x} \mathrm{~S}$ at room temperatures.

\begin{tabular}{|c|c|c|c|c|c|}
\hline Experimenters & Sample form & $\rho(r-c m)$ & $\mathrm{p}\left(\mathrm{cm}^{-3}\right)$ & $\mu\left(\frac{\mathrm{cm}^{2}}{\mathrm{v}-\mathrm{s}}\right)$ & $\begin{array}{l}\text { Scattering } \\
\text { mechanism }\end{array}$ \\
\hline [45] Hirahara ('51) & polycrystalline bulk & $2 \times 10^{-2}$ & $2 \times 10^{19}$ & 14 & impurity \\
\hline [27] Eisenmann ('52) & $\begin{array}{l}\text { polycrystalline thin } \\
\text { film }(-0.1 \mathrm{~mm})\end{array}$ & $10^{2}$ & - & -- & -- \\
\hline [48] Miyatami ('56) & single crystal & 1 & - & -- & -- \\
\hline $\begin{array}{l}\text { [53] Sorokin and Paradenko } \\
(166)\end{array}$ & $\begin{array}{l}\text { bulk; } \\
\text { polycrystalline thin } \\
\text { films }\end{array}$ & $\begin{array}{r}10^{-3} \\
0.2\end{array}$ & $\begin{array}{l}4.9 \times 10^{18} \\
5.8 \times 10^{18}\end{array}$ & $\begin{array}{r}1070 \\
5.1\end{array}$ & $\begin{array}{l}\text { acoustic } \\
\text { phonon }\end{array}$ \\
\hline [51] Abdullaev et al ('68) & single crystal & $6 \times 10^{-3}$ & $7.4 \times 10^{19}$ & 25 & $\begin{array}{l}\text { acoustic } \\
\text { phonon }\end{array}$ \\
\hline [52] Bougnot et al ('71) & $\mathrm{Cu}_{2.0} \mathrm{~S}$ & $5 \times 10^{-2}$ & -- & -- & $\rightarrow$ \\
\hline & $\begin{array}{l}\text { Cul.94s single } \\
\text { crystals }\end{array}$ & $2 \times 10^{-3}$ & $4 \times 10^{20}$ & 7 & - \\
\hline $\begin{array}{l}\text { [49] Okamoto and Kawai } \\
(' 72)\end{array}$ & single crystal & 100 & -- & 0.3 & -- \\
\hline
\end{tabular}


III. Theory

\section{A. Optical Absorption Processes}

The most fundamental optical absorption processes in semiconductors involve the excitation of valence band electrons into the conduction bands. The intrinsic absorption spectrum of a material depends on its band structure. Consequently, the study of these processes is an iavaluable tool for probing the band-structures of semiconductors. A second class of optical absorption processes is due to free carriers. These processes are important in the study of practical solar cell materials. The non-ideality of such materials results in high concentrations of free carriers which can contribute to significant sub-bandgap transitions. The study of these processes provides important information regarding the distribution and concentration of defects which are the primary sources of the free carriers and the dominant scattering meshanisms which assist in the optical transitions.

\section{Instrinsic Absorption}

a) Direct Transitions

When the valence band maximum and conduction band minimum are located at the same point in the Brillouin zone, most commonly at $\vec{k}=0$, the dominant transitions are direct ones. An example of this kind of band structure is GaAs ${ }^{[55]}$, shown in Fig. 9a. For photons with energies $h v \geq E_{g}$, the absorption coefficient is related to the energy difference by

$$
a_{D}=A_{D}\left(h v-E_{g}\right)^{1 / 2}
$$




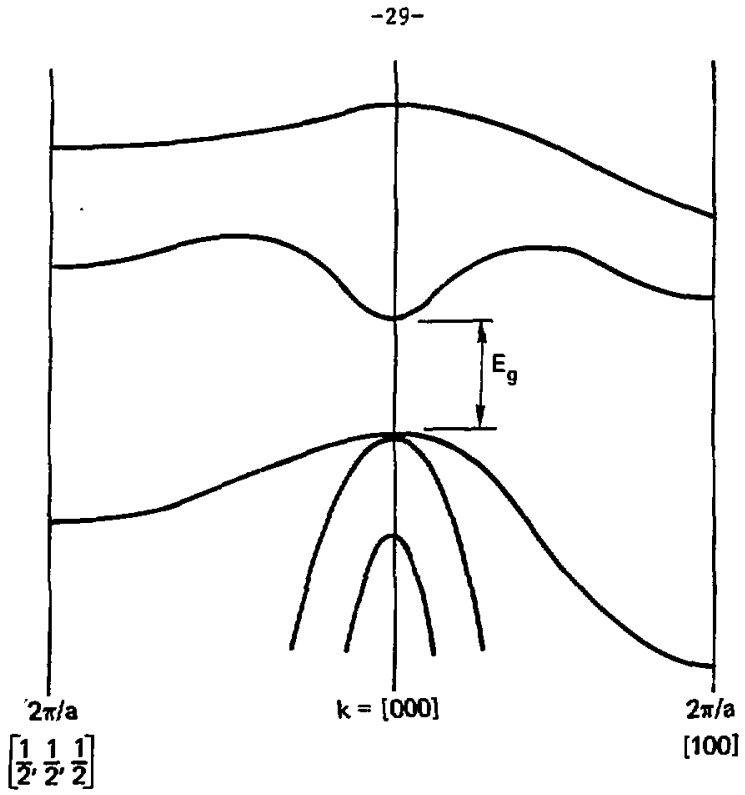

Figure 9a. Band structure of GaAs [55].

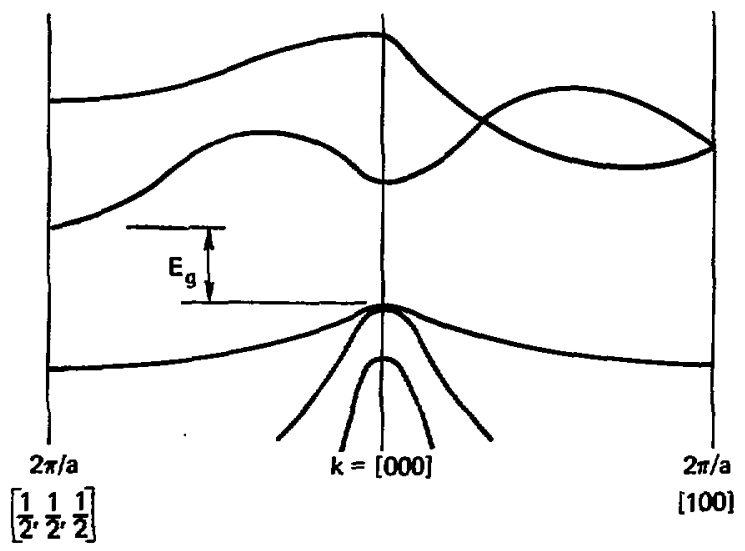

Figure 9b. Band structure of Ge [59]. 
The definition of the proportional constant $A_{D}$ and derivation of Eq. $(3-1)$ is given in Appendix II.

In some materials direct transitions are forbidden for $\vec{k}=0$ but allowed for $\vec{k} \neq 0$ by quantum selection rules. In these cases it has been shown that the absorption coefficient is given by [56]

$$
u_{D}^{\prime}=A_{D}^{\prime}\left(h v-E_{g}\right)^{3 / 2}
$$

where $A_{D}^{\prime}$ is a proportionality constant similar to $A_{D}$.

In a degenerate semiconductor with very small effective masses an anomolous shift in the absorption edge with carrier concentration known as the Burstein-Moss shift can occur. This phenomenon was first observed in studies with $\operatorname{InSb}[57,58]$ and later independent ly explained by Burstein ${ }^{[36]}$ and Moss. [37] A low effective mass is synonymous with a highly curved energy surface in k-space and low density of states as shown in Fig. 10. In InSb with $\mathrm{m}_{e}^{*}=0.03 \mathrm{~m}_{0}$ changes in degeneracy can have a dramatic effect on the observed band edge or effective bandgap $\left(E_{g}\right)$ which is approximately defined by the difference between the Fermi level and highest filled state in the valence band. The density of state effective mass can be deduced if the energy shift $(\triangle E)$ between a degenerate and non-degenerate material is known. The free carrier concentration is given by

$$
n=\int_{0}^{\Delta E} d n(E)=\frac{8 \pi}{3 h^{3}}\left(2 m^{*} \Delta E\right)^{3 / 2}
$$

where $m^{*}$ is the density of states effective mass

$$
\begin{aligned}
& \text { mo is the electron rest mass } \\
& h \text { is Planck's constant }
\end{aligned}
$$




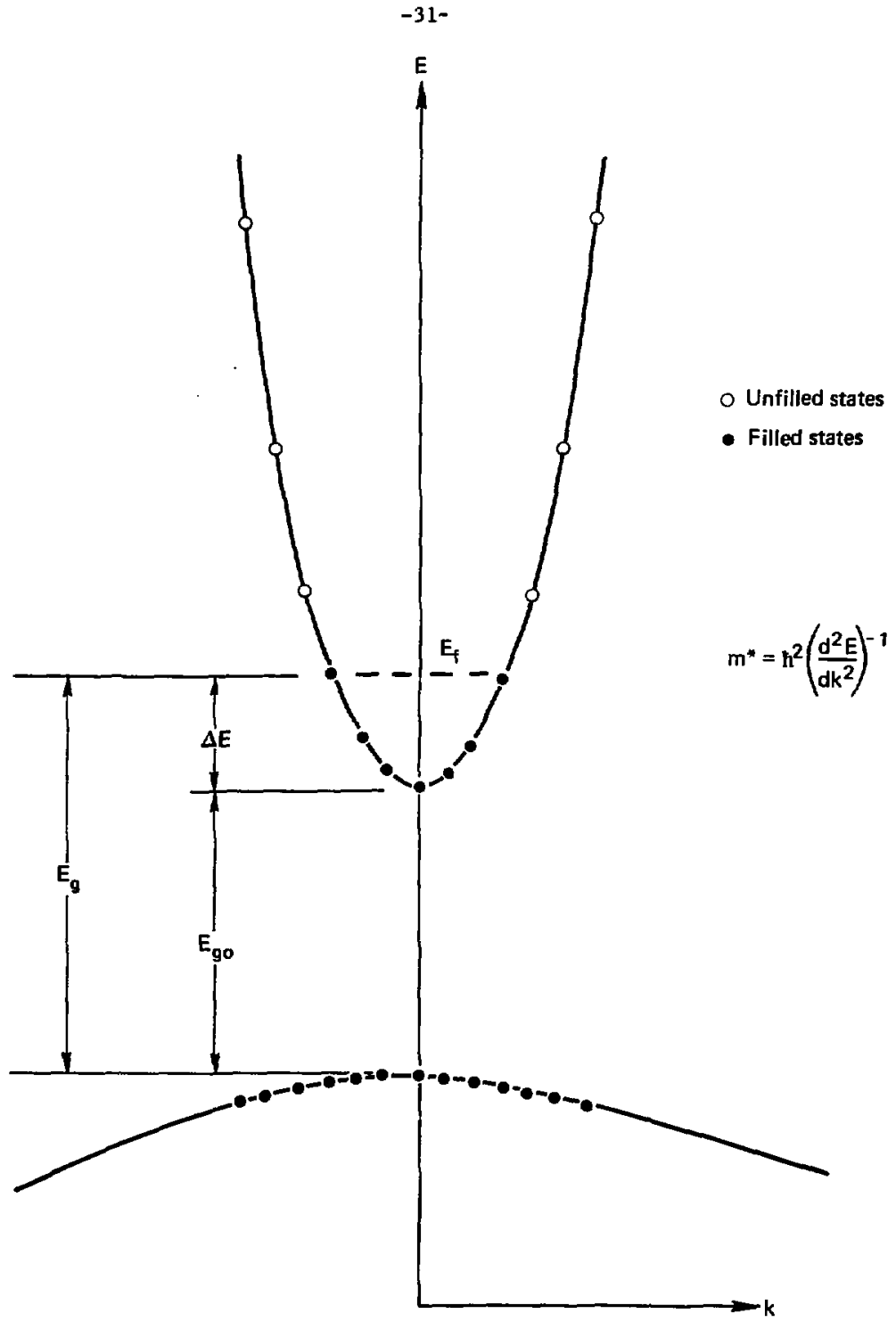

Figure 10. Burstein-Moss shift in degenerate n-type semiconductor. 
so

$$
\frac{m^{*}}{m_{o}}=3.6 \times 10^{-15} n^{2 / 3 / \Delta E} \quad(c \cdot g . s)
$$

\section{b) Indirect Transitions}

Germanium is a good example of an indirect band gap material. Its band structure ${ }^{[59]}$ is given in Fig. 9b. The threshold optical transitions are indirect, or non-vertical, ones. It involves the simultaneous emission or absorption of a phonon with the absorption of the photon. The phonon is required for momentum conservation. Indirect transitions are second order, whereas direct transitions are first order in a perturbation expansion. Consequently, the transition rates and absorption coefficient of the former are much lower than the latter. The energy difference dependence of $\alpha$ is derived in Appendix IIL and given by

$$
\begin{aligned}
\alpha_{I}=A_{I}\left[\frac{\left(h v+k_{B} \theta-E_{g}\right)^{2}}{e^{\theta / T}-1} S\left(h v+k_{B} \theta-E_{g}\right)\right. \\
\\
\left.\quad+\frac{\left(h v-k_{B} \theta-E_{g}\right)^{2}}{1-e^{-\theta / T}} S\left(h v-k_{B} \theta-E_{g}\right)\right]
\end{aligned}
$$

where $A_{I}$ is a constant,

$\theta$ is the characteristic temperature of the phonons, and $\quad s(x)$ is a step function in $x$.

In highly doped semiconductors it is possible to conserve momentum by impurity scattering ${ }^{[60]}$ or by electron-electron scattering. The absorption coefficient should then be proportional to the impurity concentration $\left(N_{I}\right)$. This has been demonstrated in degeneracely doped $\mathrm{Ge}{ }^{[60]}$ The absorption coefficient is given by 


$$
\alpha_{I}^{\prime}=A_{I}^{\prime} N_{I}\left(h v-E_{g}-\Delta E\right)^{2}
$$

where $A_{I}^{\prime}$ is a constant.

The squared dependence on the energy difference is preserved but $h$ igh impurity concentrations can regult in over an order of magnitude increase in the absorption coefficient. Data for highly doped Ge at $300^{\circ} \mathrm{K}$ are reproduced in Fig. 11. A Burstein-Moss bandgap shift also appears in the data.

\section{c) The Urbach Effect}

A third intrinsic absorption characteristic often observed in compound semiconductors is the Urbach effect. The Urbach effect takes its name from its discoverer who first observed it in $\mathrm{AgBr}$. [62] It has since been observed in semiconductors such as GaAs, [63] $\mathrm{ZnS}$, [64] and cdSe. [65] It is characterized by an absorption coefficient that increases exponentially with photon energy near the band edge. Two likely explanations have been offered.

Pankove [63] attributes the exponential dependence to the presence of a band tail with an exponential distribution of states. The case of a degenerate p-type semiconductor is illustrated in Fig. 11. As a result of high defect concentrations the interaction between defects causes broadening and merging of the energy levels into impurity bands within the gap. $[66,67]$ If the original localized states are shallow donor and acceptor states the impurity bands can merge with the normal bands to produce band tailing as shown in Fig. 12. At the same time the Fermi level undergoes a Burstein-Moss shift deeper into the valence band. A direct transition from the undistorted region below the Fermi level to a stace in the conduction band tail involves an absorption coefficient of the form ${ }^{[63]}$

$$
\alpha_{u}=A_{u}(\Delta E)^{1 / 2} e^{(h \nu-\Delta E) / E_{0}}
$$




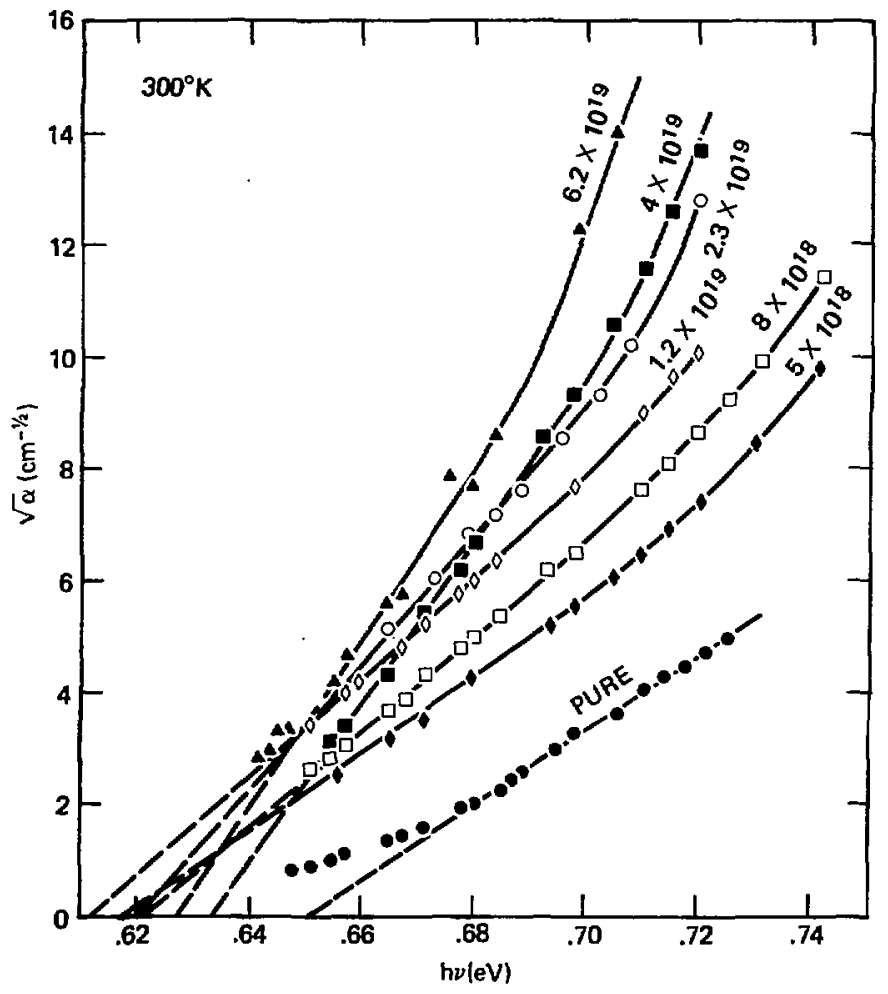

Figure 11. Absorption coefficient of degenerate Ge [60]. 
$-35-$

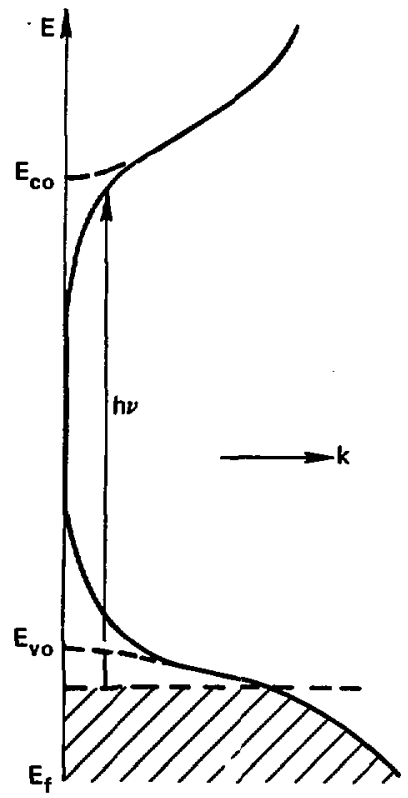

Figure 12. Band tailing in degenerate p-type semiconductor. 
where $\Delta E=E_{v o}-E_{f}$,

$E_{\text {vo }}$ is the unperturbed valence band edge,

and

$A_{u}$ and $E_{0}$ are constants.

Generally, the valence band undergoes less tailing than the conduction band. One reason is that the holes are less affected than the usually lighter electrons by charged acceptors or donors. The other is that ionized donors, which are more likely found near the conduction band, are more effective than ionized acceptors in scattering the electrons and perturbing their energy states. Given that both types of defects have equal but opposite charge, an ionized donor will attract a conduction electron, thus lowering its energy; but an ionized acceptor will repel the electron so that it will tend to stay away and remain in its unperturbed state. $[60]$

Redfield [68] proposed an alternate model to explain the Urbach effect based on field induced tunneling, similar to the Franz-Keldysh effect. $[69,70]$ When an electric field is applied to a semiconductor as in Fig. 13a it is possible for valence band electrons to tunnel into the conduction band. The tunneling probability is determined by the strength of the potential barrier. The probability has been computed by Wang [72] using a simple triangular barrier of height

$$
E_{b 1}(x)=E_{g}-q \mathscr{E}
$$

where $x$ is the direction of tunneling and applied field $(\mathscr{E})$,

and by Kane ${ }^{[73]}$ using a parabolic barrier

$$
E_{b 2}(x)=\frac{\left(E_{g} / 2\right)^{2}-(q \delta x)^{2}}{E_{g}}
$$




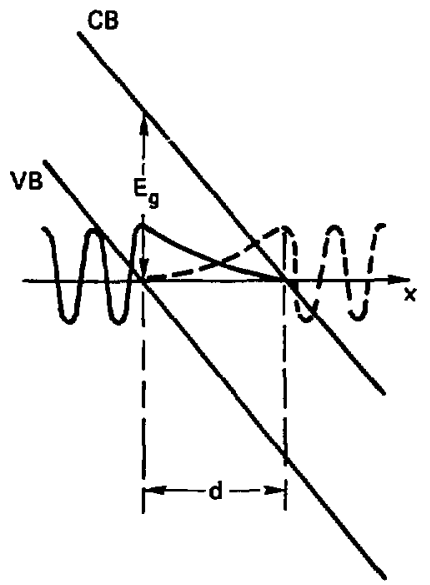

Figure 13a. Field induced tunneling [71].

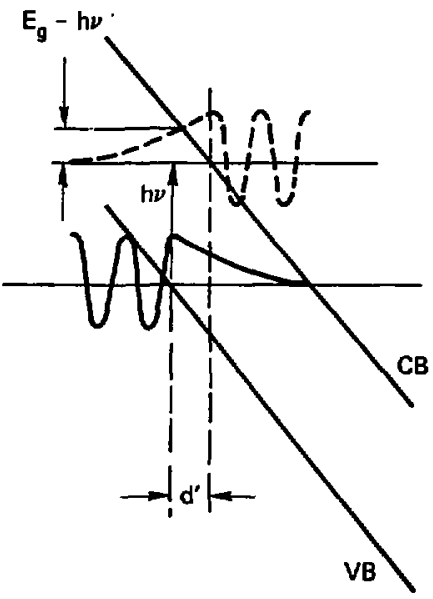

Figure 13b. Franz-Keldysh effect [71]. 
In both cases the width of the barrier is

$$
\mathbf{W}=\mathrm{E}_{\mathrm{g}} / \mathrm{q} \mathscr{E}
$$

The results are very similar. The tunneling probability depends on $\mathscr{E}$ through

$$
\mathrm{P}_{\mathrm{T}}=\mathrm{e}^{-\mathscr{E}_{\mathrm{O}} / \mathscr{E}}
$$

where $\mathscr{E}_{0}$ is proportional to $\mathrm{E}_{\mathrm{g}}^{3^{\prime} 2}$.

When a sub-bandgap photon is incident on the material the valence electron is lifted to a higher energy level, as shown in Fig. 13b, resulting in the reduction of the barrier height and width by simply replacing $E_{g}$ in Eqs. (3-8) thr ugh (3-11) with $\left(E_{g}-h v\right)$. This is the Franz-Keldysh effect. The absorption edge of a material subject to a high field is shifted to lower energy and takes on a more diffused exponential characteristic.

In the Urbach effect no external field is applied but microscopic internal local fields can be established in highly doped semiconductors. The source of the local fields may be ionized impurities, dangling bonds, polar phonons, chemical inhomogeneities, and other potential fluctuations. [74]

A numerical solution of tunneling enhanced optical absorption by Dow [75] resulted in an absorption coefficient that varies exponentially with hu which sufported the observed Urbach effect but contradicted the predictions based on a Franz-Keldysh effect. [76] Those predictions were that the absorption coefficient would have the same energy dependence as the tunneling probability or

$$
\alpha_{u} \sim e^{-\left(\left|E_{g}-h v\right| / q \mathscr{E}\right)^{3 / 2}}
$$


Dow [75] attributed the difference to the effects of the Coulomb fields which vary with $1 / r$ in contrast to the uniform applied field in a Franz-Keldysh process. The former caused more distortion and reduction of the tunneling than the latter.

In summary, the absorption coefficient is given by

$$
\alpha_{u} \sim e^{h v / E_{0}}
$$

regardless of which model is used to explain the Urbach effect. The constant $E_{0}$ has been found to be nearly equal to $k_{B} T$ for a wide range of materials. No quantitative explanation has been offered.

\section{Free Carrier Absorption}

Long wavelength or sub-bandgap photons can be absorbed by a semiconductor to excite free carriers from one energy state to another within the same band. The absorption coefficient due to free carrier absorption is a function of the number of free carriers and of the dominant scattering mechanisms. The latter dependence is due to the fact that an interaction with a third body is required to conserve momentum. The third body can be the lattice, via phonon absorption or enission, or various defects and impurities.

The relationship between the optical absorption process and scattering phenomena is contained in one of the definitions of the absorption coefficient ${ }^{[7]}$

$$
\alpha_{f}=4 \pi \sigma / \underline{\underline{n}}
$$


where $\sigma=\frac{n q^{2} \tau}{m^{\frac{1}{t}}\left(\omega^{2} \tau^{2}+1\right)}$

$\underline{n}$ is the index of refraction,

$\mathrm{n}$ is the concentration of free carriers,

$\omega$ is the frequency of the photon,

and $T$ is the lifetime of the excited state or relaxation time of the scattering process.

In the infrared and far infrared regions $\omega \tau>1$ so that

$$
\sigma=\sigma_{0} /(\omega \tau)^{2}
$$

where $\quad \sigma_{0}=\frac{n q^{2} \tau}{m^{*}}$ is the d.c. conductivity.

The majority carrier effective mass can be derived from Eqs. (3-14) and $(3-17)$

$$
m^{*}=2 n\left(\frac{\pi}{c_{n} \alpha_{f o} \sigma_{0}}\right)^{1 / 2} q^{2} .
$$

For metals, the classical Drude model, which assumes an energy independent relaxation time, gives an absorption coefficient that increases with $\lambda^{2}$. Even when the proper energy dependence is assigned to $\tau$, the conductivity and absorption coefficient are still proportional to $\lambda^{2}$ because of the peculiarity of the Eermi-Dirac distribution function. [78] But for semiconductors in which the energy dependence of $\tau$ is a function of the scattering mechanisms the analysis of $\alpha(\lambda)$ can reveal the dominant scattering mechanism. When the dominant scatcering mechanisn is acousic phonons $[79\}$

$$
\alpha \sim \lambda^{1.5} \text {; }
$$


when it is optical phonon scattering $[80]$

$$
u-\lambda^{2.5}
$$

and when it is ionized impurity scattering $[78]$

$$
\alpha-\lambda^{3} \text { or } \lambda^{3.5}
$$

\section{Temperature Effects}

When the temperature of a semiconductor is varied its absorption spectrum can change as a resulc of changes in the crystal structure, the distribution of carriers, and, in the case of the Urbach effect, the internal fields.

In most non-degenerate semiconductors the bandgaps tecrease with increasing temperature. Part of the bandgap narrowing is due to dilation of the crystal lattice. $[81,82]$ According to the energy band model based on the splitting and broadening of atomic states, Fig. 14 , the bandgap decreases with increasing interatomic separation $\left(r_{A}\right)$. But a major part of the bandgap narrowing may also be due to increased electron-phonon interactions. [83] It has been shown ${ }^{[84]}$ that the bandgaps of many common insulators and semiconductors (diamond, SiC, Si, Ge, GaAs $I n P$, and $I n A s)$ follow an empirical relationship

$$
E_{g}(T)=E_{g}(0)-\frac{c_{1} T^{2}}{T+c_{2}}
$$

where $c_{1}$ and $c_{2}$ are constants.

The average temperature coefficient $\left(\mathrm{dE}_{\mathrm{g}}^{*} / \mathrm{dT}\right)$ is on the order of $10^{-4} \mathrm{ev} /{ }^{\circ} \mathrm{K}$. 


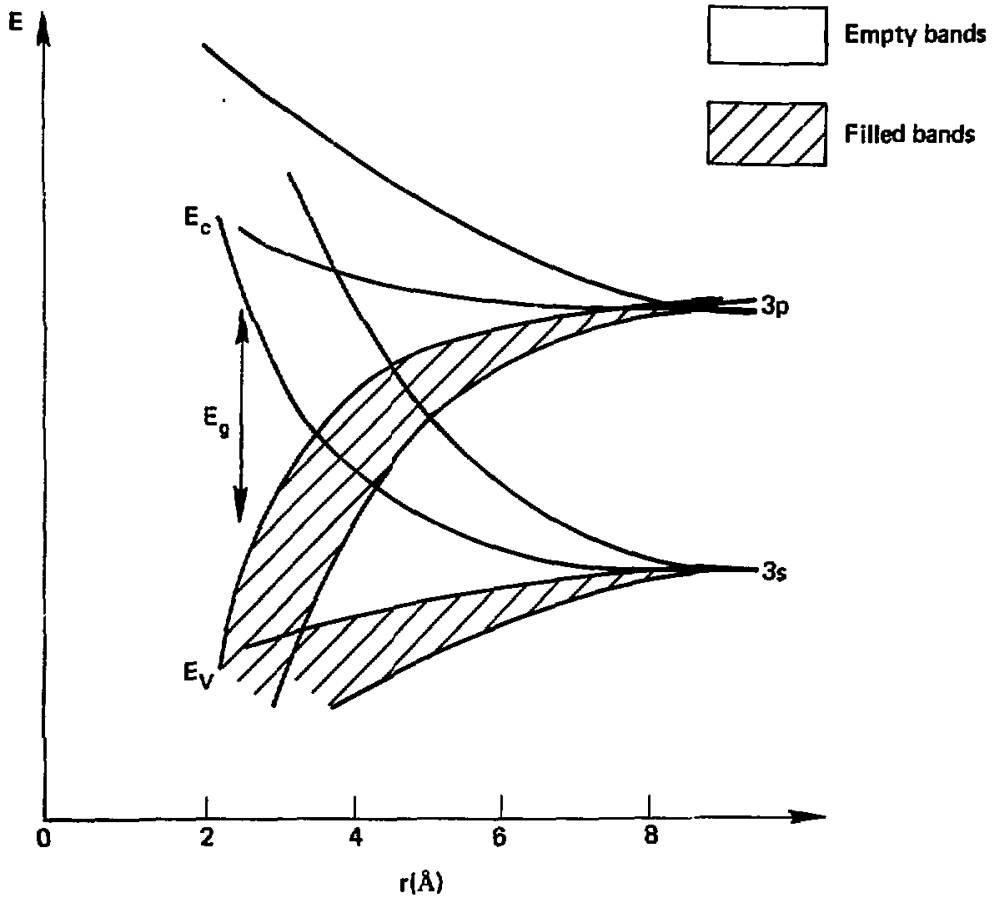

Figure 14. Splitting of $3 s$ and 3p states in Si [85]. 
Changes in observed band gaps can be induced by changes in the concentration of free carriers and distribution of states as the temperature varies. In highly doped semiconductors the increase in thermally ionized donor and/or acceptor states results in increased band tailing. So the effective band gap shrinks with increasing temperature. This has been observed in $\mathrm{Ge}^{[86]}$ and in GaAs. [87] on the other hand increased ionization may result in a greater BursteinMoss shift. The observed effect depends on which perturbation is more dominant. The Burstein-Moss shift is more likely when there is a low density state but then tailing is also more likely when the effective mass is small.

In parallel with the two explanations of the Urbach effect an alternative explanation of the bandgap narrowing in highly degenerate semiconductor is associated with increased field assisted tunneling. Increased ionized impurity concentrations lead to higher field intensities which, according to Eq. (3-11), results in higher tunneling probability. This was demonstrated in heavily doped GaA3. [88] The data is show in Fig. 15. In addition to a reduction in the bandgap there is a decrease in the slope of the absorption edge with increasing cemperature. The more pronounced tailing is due to the exponential dependence of the tunneling probability to $\left(\mid E_{g}-h \vee I / q \mathscr{E}\right)$ which is more sensitive to changes in $\mathscr{E}$ at low photon energies (i.e., large IE - hvi). 


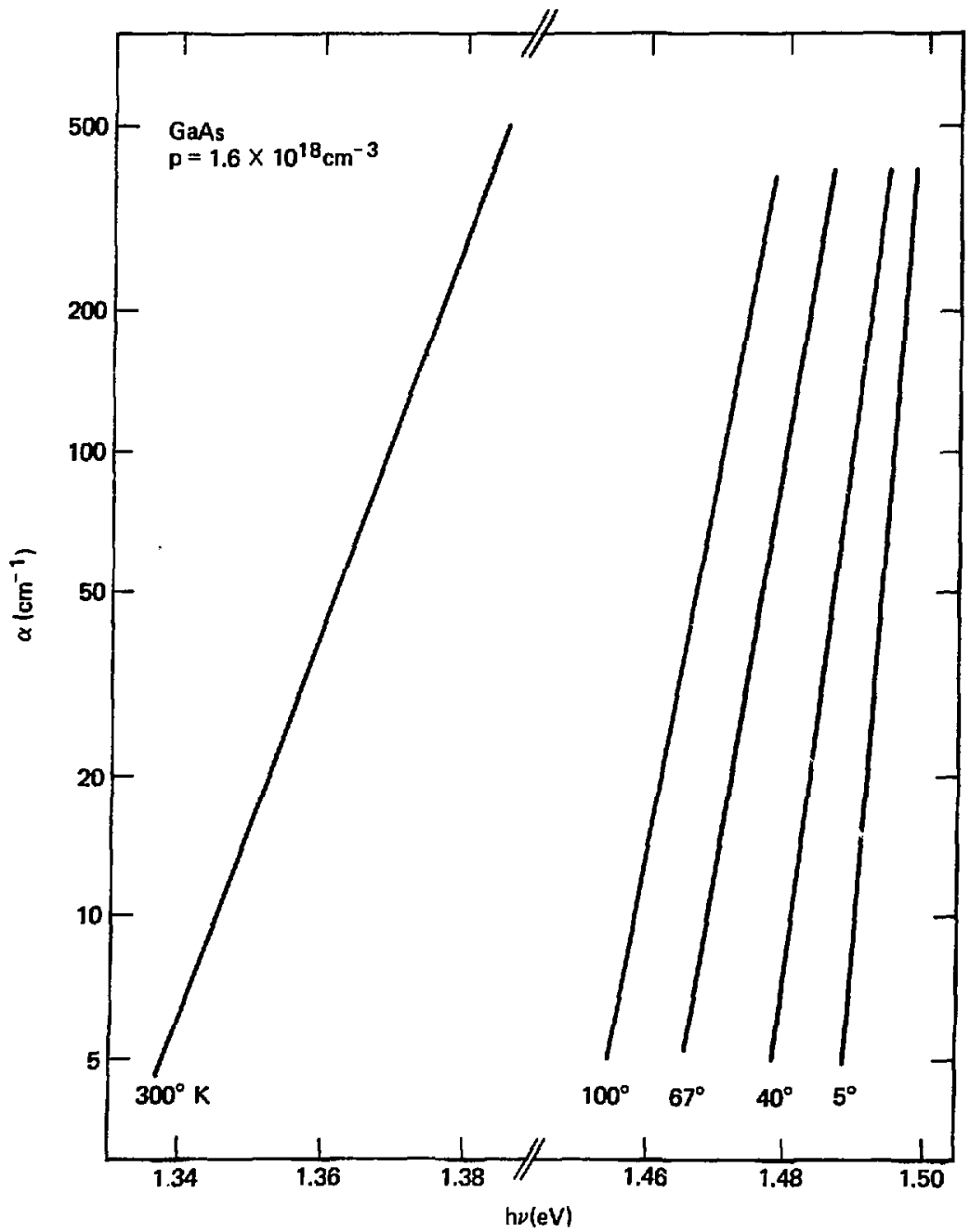

Figure 15. Temperature dependent Urbach effect in degenerate p-GaAs [8B]. 


\section{B. Mobility and Scattering Mechanisms}

Carrier mobility in an infinite solid with perfect lattice periodicity is infinite. In a real material the perfect periodicity is destroyed by the presence of various defects and by lattice vibrations. So mobility in a semiconductor is 1 imited to a finite value by these various scattering mechanisms. Five likely mechanisms for $\mathrm{Cu}_{\mathrm{x}} \mathrm{S}$ are considered here. Detail derivations of the two dominant ones are presented in Appendices IV and $V$. In addition, the effect of grain boundaries in polycrystalline materials is briefty outlined.

In most cases, when the scattering is an elastic process involving little energy exchange a relaxation time $(\tau)$ can be defined. In Appendix IV it is shown that mobility can be expressed in terms of a weighted expectation value of $\tau$

$$
\mu=\frac{q}{3 k_{B} T} v^{2} \tau
$$

where

$T$ is the temperature

$k_{B}$ is Boltzman's constant

$v$ is the velocity.

The one exception in the cases considered here is polar optical phonon scatcering which is inelastic and can involve significant energy exchange. Mobility must then be computed by more rigorous techniques. Variation principles are applied in Appendix $V$ to obtain a solution.

\section{Lattice Scattering}

a) Acoustic Phonons and the Deformation Potential

Scattering by acoustic phonons is present in all material and is due to the modulation of the lattice by lattice vibrations. Since the splitting between the conduction and valence bands is a function of 
the lattice constant as shown in Fig. 16a the modulations cause local variations in the conduction and valence band edges as shown in Fig. 16b. The change in either band edge is

$$
\Delta=\mathrm{E}_{1} \delta
$$

where $\delta$ is the lattice dilation

and $E_{1}$ is the acoustic deformation potential.

Conceptually, scattering is the reflection of the electron wave at the boundary of two local variations. Using perturbation theory Bardeen and Schockley ${ }^{[89]}$ have derived a relaxation time in terms of the deformation potential.

$$
\tau_{a . c .}=\frac{\pi h^{4} \rho_{m}}{k_{B} T}\left(\frac{v_{s}}{E_{1} m^{*}}\right)^{2}\left(\frac{m^{*}}{2 E}\right)^{1 / 2}
$$

where $p_{m}$ is the mass density,

and $v_{s}$ is the speed of sound.

Using Eq. (3-23) mobility is then

$$
\mu_{a . c .}=c_{a \cdot c \cdot} T^{-3 / 2}
$$

where $\quad c_{a . c .}=\frac{2}{3}(2 \pi)^{1 / 2} \rho_{m} \frac{v_{s}}{E_{1}} \frac{\hbar^{4}}{\hbar^{5 / 2} k_{B}^{3 / 2}}$.

b) Piezoelectric Scattering

In crystals which lack inversion symmetry acoustic vibrations can produce fluctuating dipole fields. In II-VI compounds, and particularly $\mathrm{CdS}^{[91,92]}$ the effect of the polarization field far exceeds that of the simple deformation potential. Because of the similarity between the $\mathrm{Cu}_{\mathbf{x}} \mathrm{s}$ and CdS crystal scructures, it's reasonable to expect 


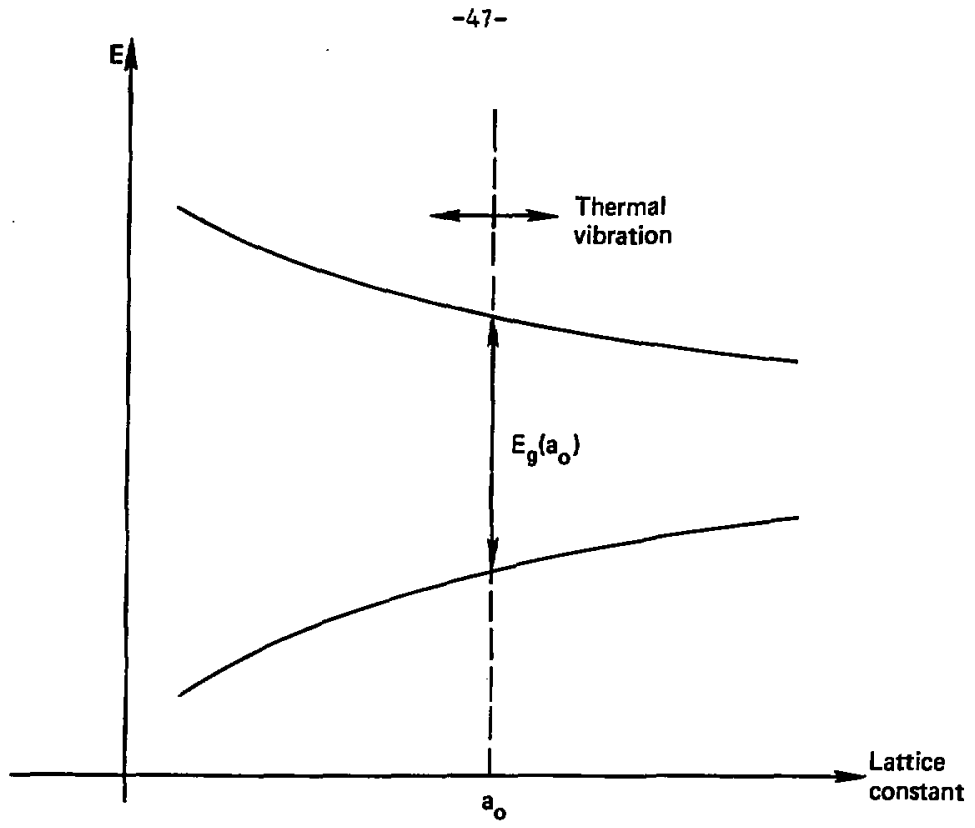

Figure 168. Changes in Eg due to thermal vibrations [90].

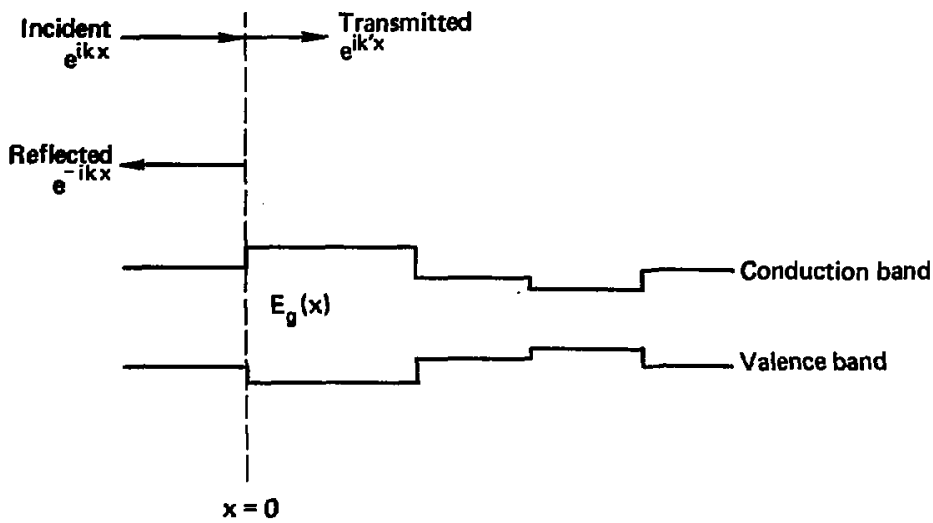

Figure 16b. Electron scattering by deformation potential [90]. 
piezoelectric scattering to play a significant role in determining the mobility of the former as well. Harrison [93] derived an expression for the mobility based on a relaxation time approximation

$$
\mu_{\text {p.z. }}=\mathrm{C}_{\mathrm{p} \cdot \mathrm{z} \cdot \mathrm{T}^{-1 / 2}}
$$

where $\quad C_{p . z .}=\frac{0.044 P_{m}}{q m^{*^{3 / 2}} k_{B}^{1 / 2}}\left(\frac{v_{s}}{\beta}\right)^{2}$

and $\quad B$ is the piezoelectric constant.

Meijer and Polder ${ }^{[94]}$ independently derived a similar expression based on essentially the same model.

\section{c) Polar Optical Phonon Scattering}

Polar optical phonon scattering is the optical mode counterpart of piezoelectric scattering. It's the dominant scattering mechanisin in many compound semiconductors at high temperatures where there is sufficient coupling between the free carriers and the high energy optical phonons. Devlin[92] and kroger et al ${ }^{[95]}$ found that the mobility data of CdS for $\mathrm{T}>150^{\circ} \mathrm{K}$ can be fitted by assuming polar optical phonon scattering as the dominant scattering mechanism. So it could also be an important determinant of room temperature mobility in Cu $\mathbf{x}$.

A relaxation time cannot be rigorously defined for this scattering mechanism because of the possibility of large energy exchange when an optical phonon is emitted or absorbed. An analytical expression for mobility can be obtained by a more exact solution of the Boltzman transport equation by the variational method outlined in Appendix $v$. Howarth and Sondheimer $[96]$ were the first to apply the method to semiconductors. Their solution confained an infinite series of modified Bessel functions. Good approximations can be obtained by considering 
the first two terms

$$
\begin{aligned}
\mu_{0 . p .} & =\frac{3 \pi^{1 / 2 h^{2} \varepsilon^{\prime}}}{2^{5 / 2} \text { * }^{3 / 2} k_{B} T^{1 / 2}} & \text { for } T>\theta \\
& =\frac{h^{2}\left(e^{\theta / T}-1\right)}{\left(2 q^{2} *^{*} k_{B} \theta\right)^{l / 2}} & \text { for } T<\theta
\end{aligned}
$$

where $\frac{1}{\varepsilon}=\left(\frac{1}{\varepsilon_{\infty}}-\frac{1}{\varepsilon}\right)$

$\epsilon_{\text {m }}$ and $\varepsilon$ are the high frequency and static dielectric constants, respectively.

$\theta=\hbar \omega_{o} / k_{B}$ is the characteristic temperature of the optical phonons.

$w_{0}$ is the frequency of the optical phonons.

The screening effect of free carriers was added by Ehrenreich, [97] resulting in

$$
\mu_{0 . P .}=c_{0 . p \cdot T^{1 / 2}\left(e^{\theta / T}-1\right)}
$$

where $\quad c_{\text {o.p. }}=1.15 \times 10^{30}\left(\frac{q}{q *}\right)^{2}\left(\frac{m_{0}}{{ }^{*}}\right)^{3 / 2} \mathrm{MV}_{\Omega_{0} \omega_{0} G^{(1)}} \mathrm{e}^{-\theta / T}$

$M$ is the reduced ion mass in grams

$V_{\Omega}$ is the volume of the unit cell in $\operatorname{con}^{3}$

$$
\begin{aligned}
\mathrm{q}^{*}= & \left(\mathrm{MV}_{\Omega} / 4 \pi\right)^{1 / 2} \omega_{0}\left(\frac{\varepsilon-\varepsilon_{\infty}}{\varepsilon_{-}}\right)^{1 / 2} \text { is the screened } \\
& \text { effective charge. }
\end{aligned}
$$




$$
\begin{aligned}
& \mathrm{G}^{(1)} \mathrm{e}^{-\theta / \mathrm{T}} \text { is a plotted function, Fig. } 17 \text {, of } \theta / \mathrm{T} \text { and } \mathrm{n} \\
& \text { expressed in terms of the plasma frequency, } \omega_{\mathrm{p}} \text {. }
\end{aligned}
$$

The plasma frequency in Fig. 17

$$
\omega_{\mathrm{p}} \equiv \mathrm{q}\left[4 \pi \mathrm{n} / \mathrm{Em}^{*}\right]^{1 / 2}
$$

is approximately equal to $\omega_{\circ}$ for a typical degenerate semiconductor with $\mathrm{n}=10^{19} \mathrm{~cm}^{-3}, \mathrm{~m}^{*} \simeq \mathrm{m}_{0}$, and $\theta \simeq \mathrm{T}$. [97] For $\theta<\mathrm{T}$, the

function may be assumed to be constant with a value near unity over a small range of temperatures.

Even though technically relaxation time cannot be defined, Frohlich and Mott $[98]$ obtained results similar to Eqs. $(3-29 a)$ and $(3-29 b)$ by using a relaxation time approach. Harrison and Hauser ${ }^{[99]}$ also showed that a reasonable approximation of the expression in Eq. (3-30) can be obtained by assuming a relaxation time. These demonstrations that a reasonable relaxation time can be contrived make it possible to apply Mathiessen's law ${ }^{[100]}$ to analyze the mobility due to several independent scattering mechanisms

$$
\mu^{-1}=\sum_{i} \mu_{i}^{-1} .
$$

One qualification on Eq. (3-29) is that considerable inaccuracy may result at cemperatures where the mobility components due to lattice scattering and impurity scattering are comparable. [101]

\section{Impurity Scattering}

a) Ionized Impurities

Ionized impurities is a general term that encompasses all point imperfections that may have a real or effective charge. This includes vacancies, interstitial and substitutional impurities, dangling bonds, and various defect complexes. The derivation of an ionized impurity 


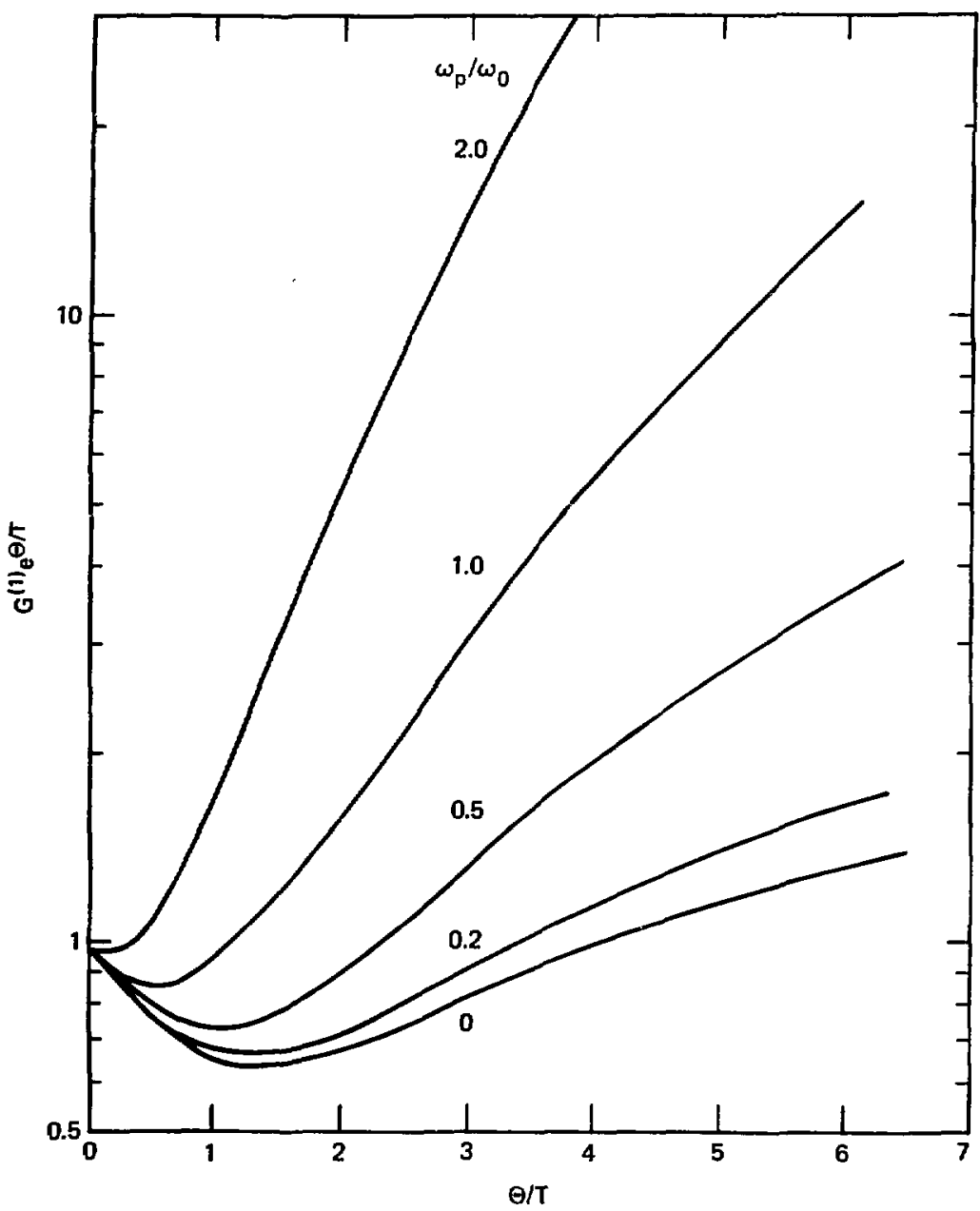

Figure 17. Screened polar optical phonon scattering function [97]. 
sc stering limited mobility using the Born approximation to obtain a scattering cross section is detailed in Appendix IV. The perturbing potential of the impurity is a screened coulomb potential

$$
V=\frac{2 q}{\epsilon r} e^{-r / r_{0}}
$$

where $z$ is the net charge,

and $r_{0}$ is the screening radius.

The screening radius is the Debye-Hueckel radius defined by Brooks ${ }^{[102]}$

$$
r_{0}^{2}=\frac{k_{B} T N_{I}}{4 \pi q^{2} n\left(2 N_{I}-n\right)}
$$

where $\mathbb{N}_{I}$ and $n$ are the ionized and total impurity concentrations, respectively.

The derivation in Appendix IV uses a more general screening radius defined entirely in terms of free carrier concentration [103]

$$
r_{0}=9.92 \times 10^{-8} \varepsilon^{1 / 2} T^{-1 / 4}\left(\frac{m_{0}}{m^{*}}\right)^{3 / 4}\left[\sigma_{-1 / 2}(n)\right]^{-1 / 2}(\mathrm{~cm})
$$

where $n=E_{E} / k_{B} T$

$$
F_{-1 / 2}(\pi) \text { is a tabulated function, given in Table } 3 \text {. }
$$

The resulting expression for mobility is

$$
\mu_{I}=C_{I} T^{3 / 2}
$$

where

$$
\begin{aligned}
& C_{I}=\frac{3.3<10^{15}}{N_{i} z^{2} \ln (B+1)} \varepsilon^{2}\left(\frac{m_{0}}{m^{\frac{k}{k}}}\right)^{1 / 2} \\
& B=\left(2 \mathrm{kr}_{0}\right)^{2} .
\end{aligned}
$$


TABLE 3. Fermi integral. [103]

\begin{tabular}{cccccc}
\hline$n$ & $S_{1 / 2(n)}$ & $S_{-1 / 2(n)}$ & $\eta$ & $S_{1 / 2(n)}$ & $F_{-1 / 2(\eta)}$ \\
\hline-4.0 & 0.0182 & 0.0181 & 3.5 & 5.458 & 2.026 \\
-3.5 & 0.0299 & 0.0296 & 4.0 & 6.512 & 2.186 \\
-3.0 & 0.0489 & 0.0481 & 4.5 & 7.642 & 2.334 \\
-2.5 & 0.0798 & 0.0776 & 5.0 & 8.844 & 2.473 \\
-2.0 & 0.1293 & 0.1237 & 5.5 & 10.11 & 2.604 \\
-1.5 & 0.2074 & 0.1933 & 6.0 & 11.45 & 2.727 \\
-1.0 & 0.3278 & 0.2940 & 6.5 & 12.84 & 2.845 \\
-0.5 & 0.5075 & 0.4312 & 7.0 & 14.29 & 2.957 \\
0.0 & 0.7651 & 0.6049 & 7.5 & 15.80 & 3.065 \\
0.5 & 1.117 & 0.8077 & 8.0 & 17.36 & 3.169 \\
1.0 & 1.576 & 1.027 & 8.5 & 18.96 & 3.270 \\
1.5 & 2.145 & 1.249 & 9.0 & 20.62 & 3.367 \\
2.0 & 2.824 & 1.464 & 9.5 & 22.33 & 3.461 \\
2.5 & 3.607 & 1.666 & 10.0 & 24.08 & 3.553 \\
3.0 & 4.488 & 1.853 & & & \\
& & & & & \\
\hline
\end{tabular}




\section{b) Neutral Impurities}

In many solar cell materials, particularly $\mathrm{Cu}_{x} \mathrm{~S}$, there is a great variety of impurities and defects with different ionization energies. Under certain conditions, such as low temperatures, a significant number of neutral impurities or defects can exist and contribute to the the scattering of free carriers. Erginsoy [104] modeled the impurity as a hydrogen atom immersed in the dielectric medium of the semiconfuctor. He found a temperature independent mobility

$$
\mu_{N}=\frac{1.43 \times 10^{22}}{\mathrm{~N}_{\mathrm{N}}} \frac{\mathrm{m}^{*}}{\mathrm{~m}_{\mathrm{o}}}
$$

where $N_{N}$ is the neutral impurity or defect concentration.

\section{Grain Boundaries and Surface Scattering}

In addition to bulk scattering processes, mobility in polycrystalline thin films is 1 imited by potential barriers at grain boundaries and scattering by free surfaces. Grain boundaries contain a high density of traps, the sources of which are dangling bonds and impurities precipitated from the bulk or absorbed from the free space. When these traps become charged with captured majority carriers a space-charge region is established in the neighborhood of the grain boundary, as shown in Fig. 18 for an n-type material. For charge neutrality the number of trapped charges per unit area is

$$
\mathbf{n}_{\mathbf{t}}=\mathbf{n} \boldsymbol{l}
$$

where $n$ is the bulk free cartier density

and is the width of the space-charge region.

From Poisson's equation the potential barrier height is

$$
\varphi=q n_{t}^{2} / 8 n
$$




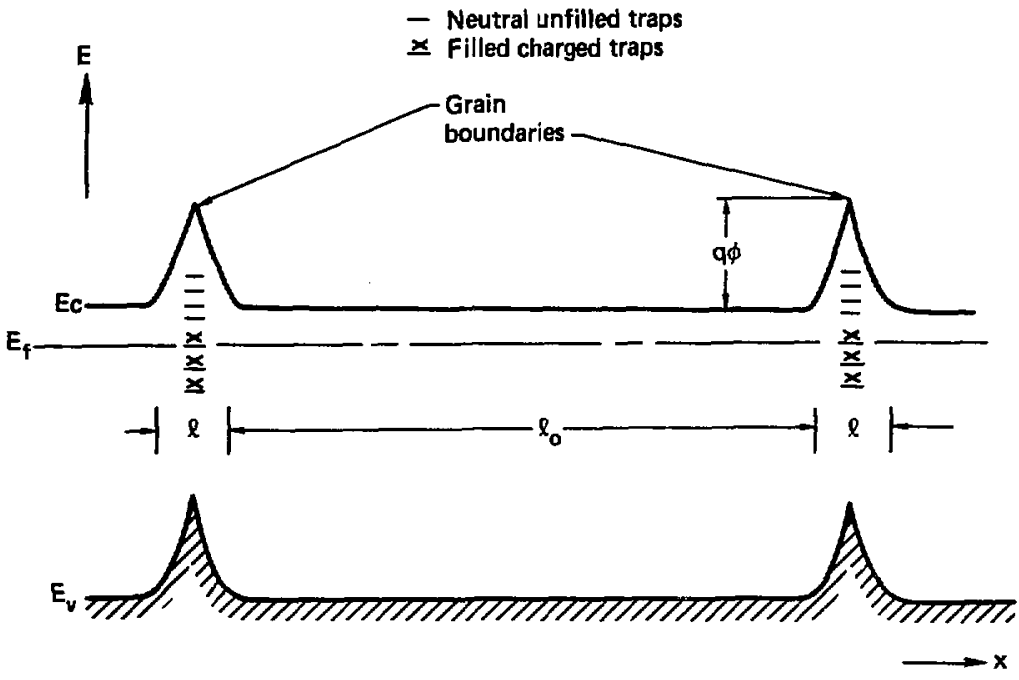

Figure 18. Barrier model of grain boundaries. 
The effective mobility is $[105,106\}$

$$
\begin{aligned}
\mu_{\text {eff }} & =\mu_{\text {bulk }}\left[1+\frac{\ell}{\ell} e^{\varphi / k_{B} T}\right]^{-1} \\
& \simeq \mu_{\text {bulk }} e^{-\varphi / k_{B} T} \text { for } \frac{\ell}{\ell} \ll e^{-\varphi / k_{B} T}=1
\end{aligned}
$$

where $\ell_{0}$ is the quasi-neutral region of the grain interior.

The actual magnitude of $\varphi$ and $\ell$ depends on the detailed distribution of the traps. At moderate bulk carrier cencentrations $\varphi$ increases with $n$ because $n_{t}$ is nearly proportional to $n$. But, at very high carrier concentration, frequently encountered in solar cell materials, $n_{t}$ saturates and both $\varphi$ and $\ell$ decrease with increasing $n$. In the limit of high degeneracy $\left(n>10^{18} \mathrm{~cm}^{-3}\right)^{[105]}$ such as the case of cu $\mathrm{s}$

$$
\mu_{\text {eff }} \simeq \mu_{\text {bulk }} \cdot
$$

Free surfaces contain a high density of defects which can 1 imit the effective carrier mobility in a material by acting as scattering centers and by changing the conductive characteristics of the surface layer. The effects are especially important in thin films because the surface layer may be a significant part of the thin film volume. Treating the surface and bulk scattering as independent processes Mathiessen's law can be applied

$$
\frac{1}{\tau_{f}}=\frac{1}{\tau_{b}}+\frac{1}{\tau_{s}}
$$

where $\tau_{f}, \tau_{b}$ and $\tau_{s}$ are the relaxation times for the film, bulk, and surface, respectively. 
Many et $\underline{a 1}^{[107]}$ defined $\tau_{s}$ by

$$
\tau_{s}=\left(d / \lambda_{s}\right) \tau_{b}
$$

where $d$ is the film thickness

and $\lambda_{s}$ is the mean free path due to surface scattering.

Then the free mobility, $\mu_{f}$, is related to the bulk mobility, $\mu_{b}$, by

$$
\mu_{\mathrm{f}}=\frac{\mu_{\mathrm{b}}}{1+\left(\lambda_{\mathrm{s}} / \mathrm{d}\right)}
$$

Taking into consideration the effects of specular versus random surface scattering and surface band bending $\{108,109\}$

$$
\frac{\mu_{f}}{\mu_{b}}=\frac{1+(1-f) \frac{\lambda_{s}}{d}\left[1-e^{\varphi_{S} / k_{B} T}\right]}{1+(1-f) \frac{\lambda_{s}}{d}}
$$

for a surface depletion layer

and

$$
\frac{\mu_{F}}{\mu_{b}}=\frac{1}{1+(1-f) \lambda\left[\frac{1}{L}+\frac{1}{d}\right]\left(1+\frac{\varphi_{S}}{k_{B} T}\right)^{1 / 2}}
$$

for a surface accumulation layer

where $f$ is the fraction of surface scattering that is specular, $\mathrm{L}_{c}$ is the depth of the accumulation layer, and $\varphi_{s}$ is the surface potential.

The surface potential is negative for a depletion layer. Just as in the grain boundary case when the material is highly degenerate, $\varphi_{s}$ and $\lambda_{s}$ are very small so that

$$
\mu_{\mathrm{f}}=\mu_{\mathrm{b}}
$$


IV. Experimental Methods

A. Sample Preparation

1. Traditional Deposition Techniques

$\mathrm{Cu}_{\mathrm{X}} \mathrm{S}$ can be prepared in numerous ways, as evidenced by the range of sample types described in Section II. The two methods that have had the greatest success in producing efficient cells are the so-called "wet" process, developed mainly by the Clevite Corporation, and the "dry" process, developed by the Philips Laboratory. Both of these methods involve the conversion of a cds substrate to $\mathrm{Cu}_{\mathrm{x}} \mathrm{s}$ by an ion exchange process.

In the "wet" process the $\mathrm{CdS}$ is dipped for several minutes in a hot $\left(90^{\circ} \mathrm{C}\right)$ aqueous solution of $\mathrm{CuCl}$. Ideally, the following reaction occurs:

$$
\mathrm{CdS}+2 \mathrm{Cu}^{+}+\mathrm{Cu}_{2} \mathrm{~S}+\mathrm{Cd}^{+2}
$$

Unfortunately, the final product is usually a mixture of $\mathrm{Cu}_{\mathrm{x}} \mathrm{S}, \mathrm{Cu}^{+}$, and $\mathrm{Cd}^{+2}$. Broad concentration gradients are established by the interdiffusion of the components, resulting in reduced junction collection fields, or even shorts through the Cds. $[11,110]$

In the "dry" process, CuCl is evaporated onto the CdS. The sample is heated to $180^{\circ} \mathrm{C}$ for several minutes in a reducing or inert atmosphere to drive the following reaction to the desired degree of completion:

$$
\mathrm{CdS}+2 \mathrm{CuCl}+\mathrm{Cu}_{2} \mathrm{~S}+\mathrm{CdCl}_{2}
$$


The excess CuCl and $\mathrm{CdCl}$ are dissolved in water or alcohol. The absence of excess $\mathrm{Cu}$ and $\mathrm{Cd}$ ions produces a very sharp junction between the converted $\mathrm{Cu}_{2} \mathrm{~S}$ and Cds. Still, there remains the problem of nonstoichiometry which can occur when $\mathrm{CuCl}_{2}$ is present and takes the place of the monovalent compound $\mathrm{CuCl}$ in the above reaction. [40]

Reactive sputtering of $\mathrm{Cu}_{\mathrm{x}} \mathrm{S}$ was developed by the Electronics Materials group at Lawrence Livermore Laboratory as an alternate approach to controlling the stoichiometry of $\mathrm{Cu}_{\mathrm{x}} \mathrm{S} \cdot \mathrm{Cu}_{2.0} \mathrm{~S}$ can be obtained by proper choices of sputtering parameters. This was verified by $x$-ray diffraction and supported by microprobe analysis and optical transmission characteristics. [26] Epitaxial growth on single crystal CdS has been demonstrated. Cells with efficiencies of $4 \%$ have been made by sputtering $\mathrm{Cu}_{x} \mathrm{~S}$ onto vapor-deposited CdS substrates. [111] If an abrupt junction is desirable for high collection fields, sputtering should be an improvement over the two conversion processes which, inherently, require intermixing of $\mathrm{Cu}$ and $\mathrm{Cd}$.

In addition to the control of stoichiometry, the sputtered films are very dense (e.g., pin hole free layers as thin as $0.2 \mu \mathrm{m}$ can be attained), highly uniform, and have highly specular surfaces. Unlike the two conversion processes, the sputtering method can be used to deposit films on glass slides. Having an isolated film of $\mathrm{Cu}_{x} \mathrm{~S}$ not only makes it possible to measure certain properties unambiguously (e.g., the Hall effect would be impossible to measure and the interpretation of optical absorption complicated with a cdS substrate), but it also allows one to manipulate independent variables in a more controlled manner (e.g., the effects of heat treatment on $\mathrm{Cu}_{\mathrm{x}} \mathrm{s}$ ). All of these desirable attributes of reactively sputtered films were exploited in the course of this work. 
2. The Sputtering Process [112]

Sputtering is a form of glow discharge. Fig. 19 is a diagram of tne principal regions of a d.c. glow discharge. The target source material serves as the cathode and the substrate as the anode; an inert sputtering gas 1 ies between the two electrodes. Glow discharge is initiated when the field in the gas is sufficiently high to cause breakdown. The electrons are quickly collected by the anode while the much heavier ions migrate slowly towards the cathode. The resulting positive space charge, located mainly in the Crookes dark space, intensifies the field between it and the cathode while shielding the anode. Ionization is increased and sustained by electron emission from the cathode. Subsequent radiative recombination is responsible for the luminescence in the various glow regions. Electrons in the Aston dark space have too little energy while those in the Crookes dark space are moving too fast to have an appreciable collision cross-section with the gas atoms. In the cathode glow and negative glow regions there is a plentiful supply of electrons and ions with well matched energies for ionizing collisions. Most of the ionization takes place in the Crookes dark space. Beyond the negative glow region is the Faraday dark space. It is a relatively inactive region because the electrons here do not have sufficient energy for ionization, having lost most of it in the negative glow region. The positive colum contains electrons which have re-acquired enough energy from the weak field to cause limited excitations. The luminosity of this region is much less intense than that of the negative glow region.

In sputtering, the alode is placed as close as possible to the cathode in order to maximize yield. The positive column and much of the Faraday dark space are removed. In a normal glow discharge the voltage is independent of the current or power input. The cross-sectional area of the glow discharge is less than that of the cathode. As the power input increases, the glow discharge widens to accomodate the larger current. As long as the current density is unchanged, the voltage 

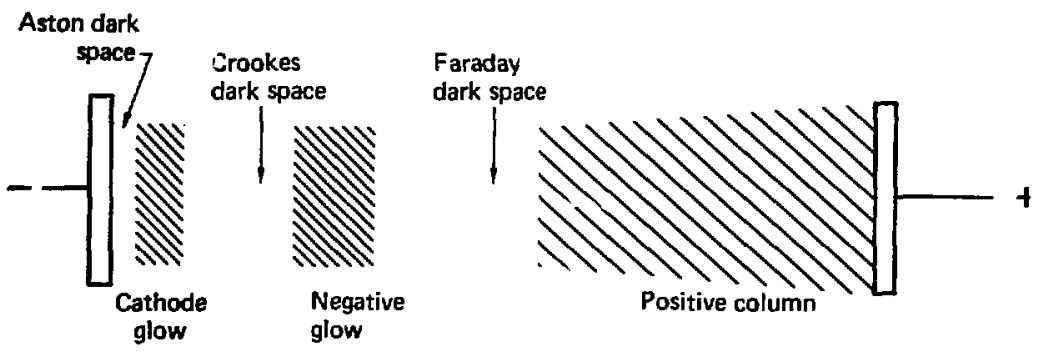

Figure 19. Principle regions of glow discharge. 
remains constant. If the power is increased to the point that the cross-sectional area of the glow discharge is equal to that of the cathode, then further increases must result in an increase in current density and voltage. Glow discharge under these conditions is "abnormal". Sputtering is a strongly abnormal glow discharge. A large flux of highly energetic ions is needed to eject bound target atoms at a useful rate.

The actual sputtering process is one of momentum transfer involving the bulk target as well as the ejected surface atom an:i the incident ion. A simple model proposed by Pease described the process in terms of radiation damage theory. [113] A high energy ion iucident on the target produces a "primary knock-on" atom. It moves into the bulk, producing a series of secondary knock-ons. Some of the secondaries may have the appropriate momentum vector to be sputtered from target. The yield is:

$$
Y=\left(\sigma_{p_{A}} p^{2 / 3}\right)\left(\frac{\bar{E}}{4 E_{d}}\right)\left\{1+\left[\frac{\ln \left(\bar{E} / E_{s}\right)}{\ln 2}\right]^{1 / 2}\right\}
$$

where $a_{p}$ is the collision cross-section of the primary knock-on,

$D_{A}$ is the atomic density,

$\bar{E}$ is the average energy of the primary knock-on,

$E_{d}$ is the energy needed to displace an atom from its lattice site,

$E_{s}$ is the sublimation energy.

The first factor is the number of atoms that a knock-on hits in passing through a layer of atoms. The second represents the number of atoms that are displaced with the momentum vector in the right direction. The last is the number of atomic layers that can contribute to sputtering with atom having sufficient energy to escape the target. The yield 
depends on the energy and mass of the incident ions through $\bar{E}$ as well as on target material properties such as $E_{d}, E_{S}$ and $\rho_{A}$.

The threshold energy for sputtering any target material is about $4 E_{s},[114]$ but for practical yields, ions with incident energies of about $1 \mathrm{keV}$ are used. The energy of ejected atoms is on the order of several electron volts which is much higher than that of evaporated atoms. [115] The higher deposition energy enharces adhesion and, in some cases, improves the crystallinity of the film.

When a reactive gas is present in the plasma, a compound of the cathode material and the gas can be deposited on the substrate. There is considerable debate about where the reaction actually takes place, but in the case of reactively sputtered $\mathrm{Cu}_{x} \mathrm{~S}$ in $\mathrm{H}_{2} \mathrm{~S} / \mathrm{Ar}$, the evidence seems to point to the substrate. The fact that no significant amount of $\mathrm{Cu}_{x} \mathrm{~s}$ is found on the originally pure copper target, even after years of use and at high $\mathrm{H}_{2} \mathrm{~s}$ partial pressures, rules out the cathode as the reaction site. Reaction can occur in the gas, but conservation of monentum and energy, with the addition of the heat of formation, requires the presence of a non-reactive third body. Under these conditions, the probability of $\mathrm{Cu}_{x} \mathrm{~S}$ formation in the plasma which contains less than $1 \mu \mathrm{m}$ partial pressure of $\mathrm{H}_{2} \mathrm{~S}$ is very low. But at the substrate, the presence of the massive anode removas the problems associated with momentum and energy conservation. The negatively charged sulfur ions are also much more abundant near the anode. Finally, the fact that the chemical composition is a sensitive function of substrate temperature (formation of $\mathrm{Cu}_{2.0} \mathrm{~S}$ occurs over a broader range of pressures centered at lower $\mathrm{PP} \mathrm{H}_{2} \mathrm{~S}$ for heated substrates) [26] supports the conclusion that the reaction takes place at the substrate.

The sputcering system used for preparing the $\mathrm{Cu}_{\mathrm{x}} \mathrm{S}$ samples was manufactured by Varian. It contains multiple targets and can be operated in any one of several modes. A functional schematic of the normal deposition wode is presented in Fig. 20. The input network is a 


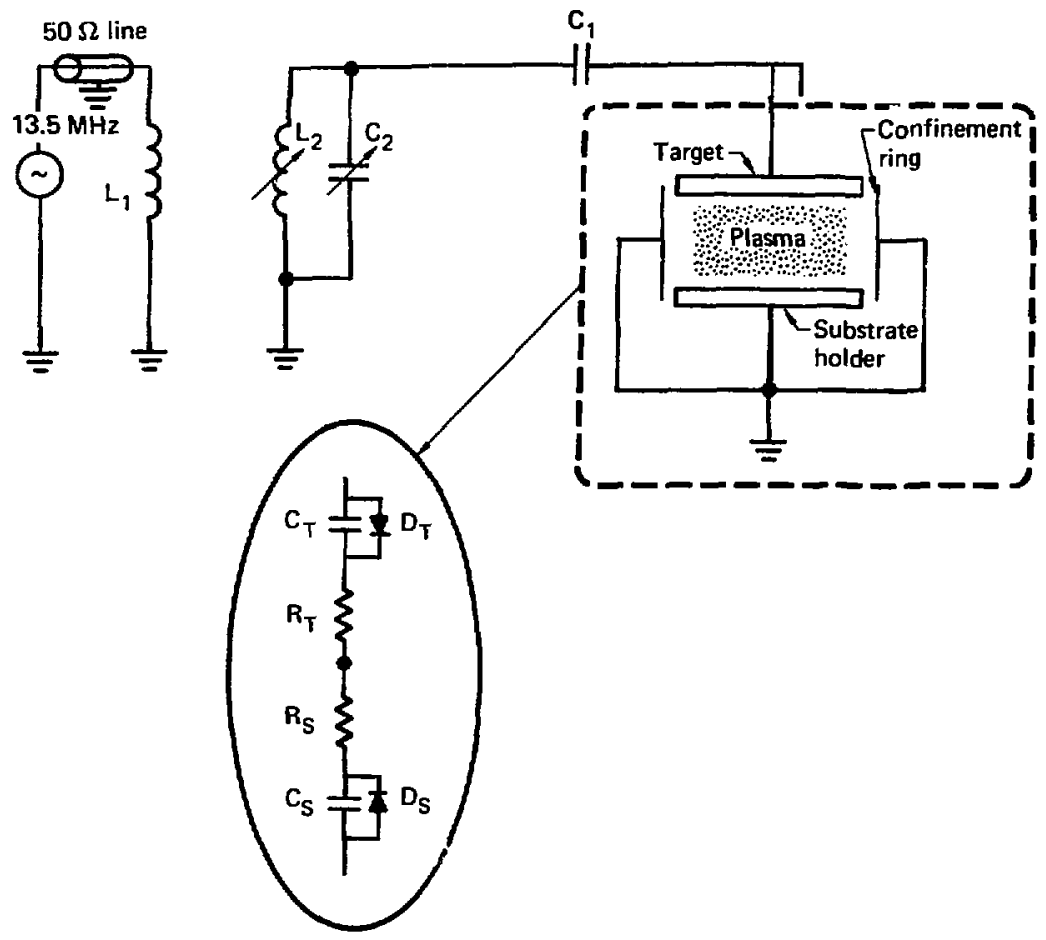

Figure 20. Functional schematic of rf sputtering system. 
13.56 MHz rf generator with $50 \Omega$ impedance. The parallel inductor $\mathrm{L}_{2}$ and capacitor $\mathrm{C}_{2}$ rake up the tuning circuit for matching the load impedance under various sputtering conditions.

A $1 \mathrm{kV}$ self-bias is established between the target and ground as a result of the electrical properties of the ionized gas and the geometry of the sputtering chamber. $[116]$ Because of the low ion mobility, an essentially static positive space charge is set up in the presence of the $r f$ input. The electrons, however, can respond very quickly to the rf and are easily collected by an electrode whenever it becomes positive with respect to the plasma. The difference between the ionic and the electronic conductance of the dark spaces at the target and the substrate is represented by the two diodes, $D_{T}$ and $D_{S}$, respectively. Each of these diodes forms a clamping circuit with $C_{1}$. The potential of the target is always negative with respect to the plasma, while that of the substrate is always positive.

The sum of the two self-biases is equal to half the peak to peak $r f$ voltage. The actual voltage distribution is given by:

$$
\frac{V_{T}}{V_{S}}=\frac{c_{S}}{c_{T}}
$$

where $v_{T}$ and $v_{S}$ are the voltages across the target and substrate dark spaces, respectively, and $\mathrm{C}_{T}$ and $\mathrm{C}_{S}$ are the corresponding capacitanises. Assuming a parallel plate configuration for the dark spaces,

$$
C=A / D
$$

where $A$ and $D$ are the cross-sectional araa and thickness of the dark space. The space charge limited ion current density is:

$$
J=\frac{k v^{3 / 2}}{D^{2}}
$$


where $D$ is the thickness of the space charge and $k$ is a proportionality co:stant. At steady state the ionic currents through the two dark spares are equal. Combining Eqs. (4-4), (4-5) and (4-6) gives:

$$
\frac{V_{T}}{V_{S}}=\left(\frac{A_{S}}{A_{T}}\right)^{2} .
$$

Since the substrate is grounded along with various shucters, guard $r$ ings, and the metal bell jar, $A_{S}$ consists of all these surfaces. Consequently, practically the entire self-bias is across the target dark space.

The glow region in $r f$ sputtering is essentially the same as in $d . c$. sputtering. The only difference between the two processes is that the electrons spend more time in the gas where they are accelerated in alternating directions with $\mathrm{rf}$. Higher sputter yields can occur because of greater ionization. Lower gas pressures can be used, thus reducing the possibility of trapping unwanted Ar in the deposited film. Film quality can also be improved by the presence of the small rf voltage across the substrate dark space. The agitating effect on the substrate can help certain growth kinetics.

The vacuum system consists of an Airco turbomolecular pump as the high vacuum component and a mechanical pump with a liquid nitrogen trap to rough out the sputtering chamber and vent the turbo pump. A pre-mixed sputtering gas, consisting of $7 \% \mathrm{H}_{2} \mathrm{~S}$ in $\mathrm{Ar}$, was introduced via an automatically controlled valve that adjusted the flow to maintain the desired absolute gas pressure in the bell jar. A Granville Philips Series 260 controller and a Baratron diaphraw-type pressure gauge were used. The chosen pressures ranged from 10 to $30 \mu \mathrm{m}--$ the higher pressures for more sulfur-rizi samples. The high vacuum was throteled whenever the sputtering gas was introduced. 


\section{Sample Processing Procedure}

Cu ${ }_{x}$ films were deposited on $1^{\prime \prime} \times 1^{\prime \prime}$ glass slides. The substrates were cleaned in several steps. They were first soaked in acetone for at least 15 minutes to remove the anti-reflection coaring. This was followed by a thorough scrubbing with "Labtone" glass cleaner from Van Waters and Rogers to remove gross contaminates. They were rinsed with de-ionized water and electronic grade methyl alcohol. After drying in air they were soaked in a hot vapor of isopropyl alcohol. If the vapor evaporated off the slides without beading they were passed onto the last stage, otherwise the slides had to be reprocessed. The last step was a vacuum bake out in the sputtering chamber at its base pressure for at least $1 / 2$ hour at $100^{\circ} \mathrm{C}$. The substrate temperature was maintained at this temperature during the entire subsequent sputtering process. Prior to deposition the $\mathrm{Cu}$ target was cleaned by sputtering onto a metal shutter for about 10 minutes.

The actual deposition time was from 45 minutes to 1 hour, which resulted in film thicknesses of about 1 m. The actual thickness was determined after the samples had undergone all the planned experiments. A scratch was made down to bare glass in the center of the sample with the tip of a metal tweezer. A Gould Surfanalyzer was used to measure the depth of the scratch.

Samples which were-to undergo Hall effect measurements were deposited on slightly modified substrates. Grooves, 1 m in depth, were cut into the $2 \mathrm{~mm}$ thick glass slides so that the center $1 \mathrm{~cm} \times 1$ ca area could be broken off easily to fit into the narrow sample holder. A bare substrate and a sputtered sample in its various forms is presented in Fig. 2 la,b,c,d. The edges of the sputcered sample were masked by the substrate holder. 


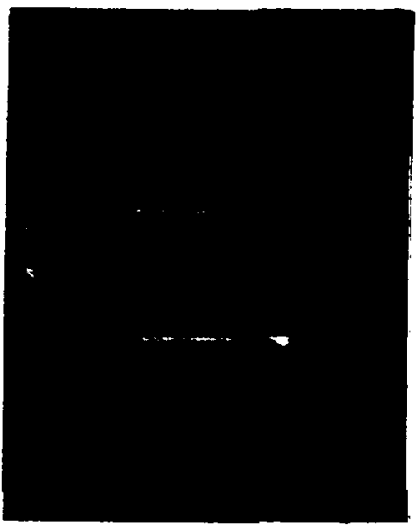

(a) bare glass substrate

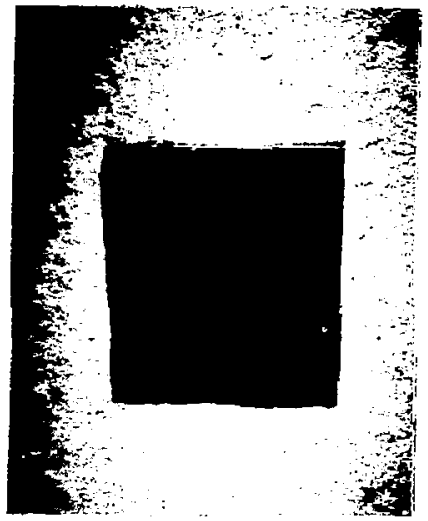

(c) $\mathrm{Cu}_{\mathrm{x}} \mathrm{S}$ with $\mathrm{Au}$ contact pads

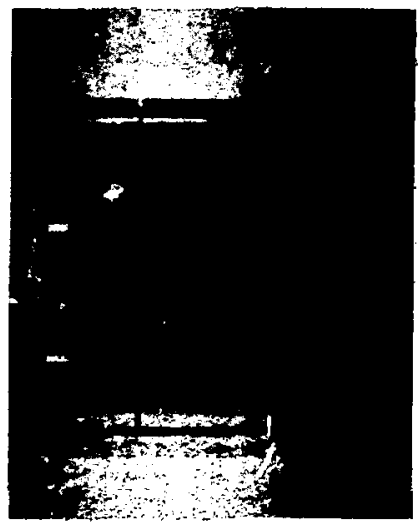

(b) sputtered $\mathrm{Cu}_{x} \mathrm{~S}$

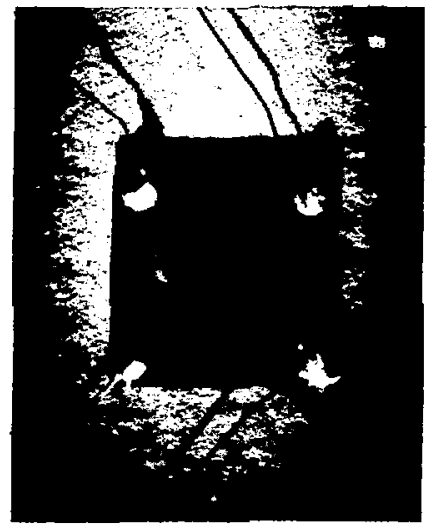

(d) complete Hall sample

Figure 21, $\mathrm{Cu}_{\mathrm{x}} \mathrm{S}$ samples. 
In order to study the effects of heat treatments, the Cu $\mathrm{S}$ films were placed in preheated furnaces $\left(180^{\circ} \mathrm{C}\right)$ containing either air or a flow of $\mathrm{H}_{2} / \mathrm{Ar}$. The air heat treatments were done in a small Thermolyne furmace while the $\mathrm{H}_{2} / \mathrm{Ar}$ treatments were done in a sealed horizontal tube furnace shown in Fig. 22. Special care was taken in the latter case to avoid heat treating the samples in residual oxygen. A sample is first placed in the cold zone $\left(T<75^{\circ} \mathrm{C}\right)$ of the tube furnace. After thoroughly flushing the system with a high flow of $\mathrm{H}_{2} / \mathrm{Ar}$ the sample is slid into the hot zone. At the end of the heat treatment the sample is slid back into the cold zone and allowed to cool. When the sample temperature reached $\mathrm{T}<75^{\circ} \mathrm{C}$ the system was opened to air and the sample removed. 


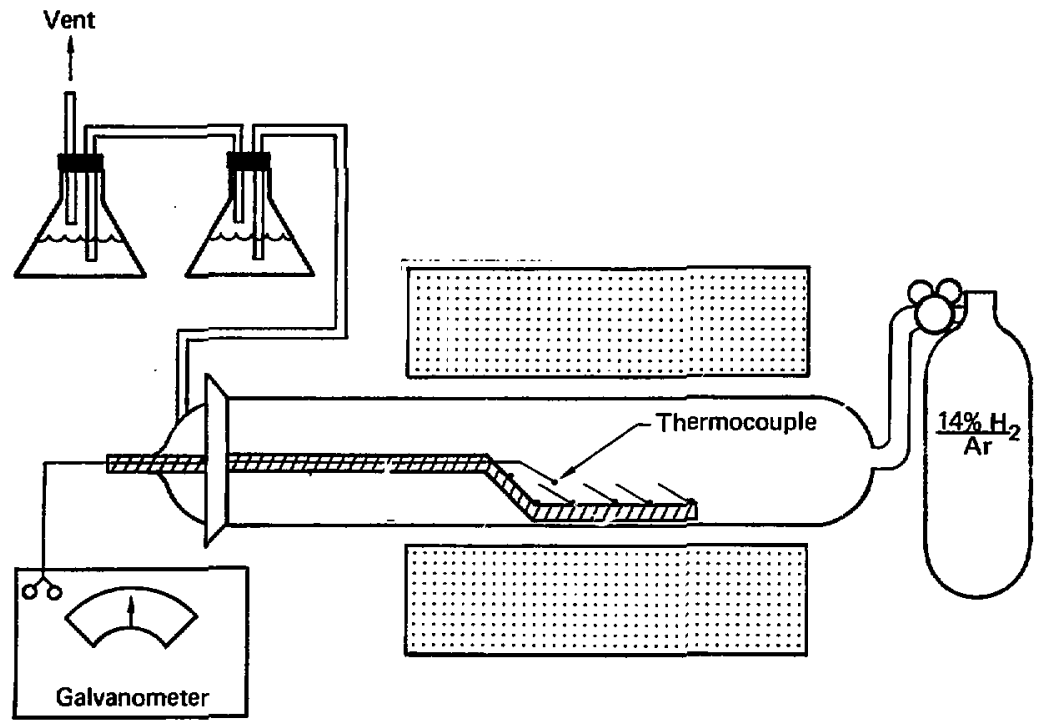

Figure 22. $\mathrm{H}_{2}$ /Ar heat treatment furnace. 


\section{B. Optical Measurements \\ 1. Instrumentation}

The transmittance (T) and reflectance (R) of the sputtered $\mathrm{Cu}_{x} \mathrm{~s}$ films in the near infrared $(0.6-2.6 \mu \mathrm{m})$ were measured with a coleman PS-3T Spectrophotometer. A functional diagram of the instrument is shown in Fig. 23. It uses a tungsten lamp 1 ight source and a rotating prism monochromator. A single beam coming out of the monochromator is deflected alternately into the sample and reference path by a rotating mirror that is half silvered and half transparent. The beam is recombined after it passes through the sample chamber by a second mirror rotating in phase with the first. The combined bean is finally deflected to a Pbs detector. A processing network amplifies, sorts, and converts the alternating sample and reference signals into a recorded plot.

A special module is inserted in the beam path for reflectance measurements. It consists of a pair of mirrors, $M_{1}$ and $M_{2}$, oriented at $45^{\circ}$ with respect to the sample and normal beam path. Mirror $M_{1}$ diverts the incident beam to the sample and mirror $\mathrm{M}_{2}$ collects the reflected 1 ight from the film and directs it to. the detector. The reference beam had to be apertured by $s 1$ it $s_{1}$ to correct for losses in the sample beam path. The slit size is adjusted so that the appropriate signal is recorded for a reference sample, a glass slide with a blackened back surface and a known index of refraction of $n=1.52$. A 11 reflectance data had to be corrected for the absorption band centered at $0.84 \mu m$ due to the aluminum mirror coating on $M_{1}$ and $M_{2}$.

A specially constructer dewar was used to make transmittance measurements at low temperatures. A sketch of the dewar is shown in Fig. 24. A sample film is mounted on the slotted cu sample holder with silicone thermopaste. When the bottom cap, which is part of the outside wall of the dewar, is put into position the 1" diameter quartz 


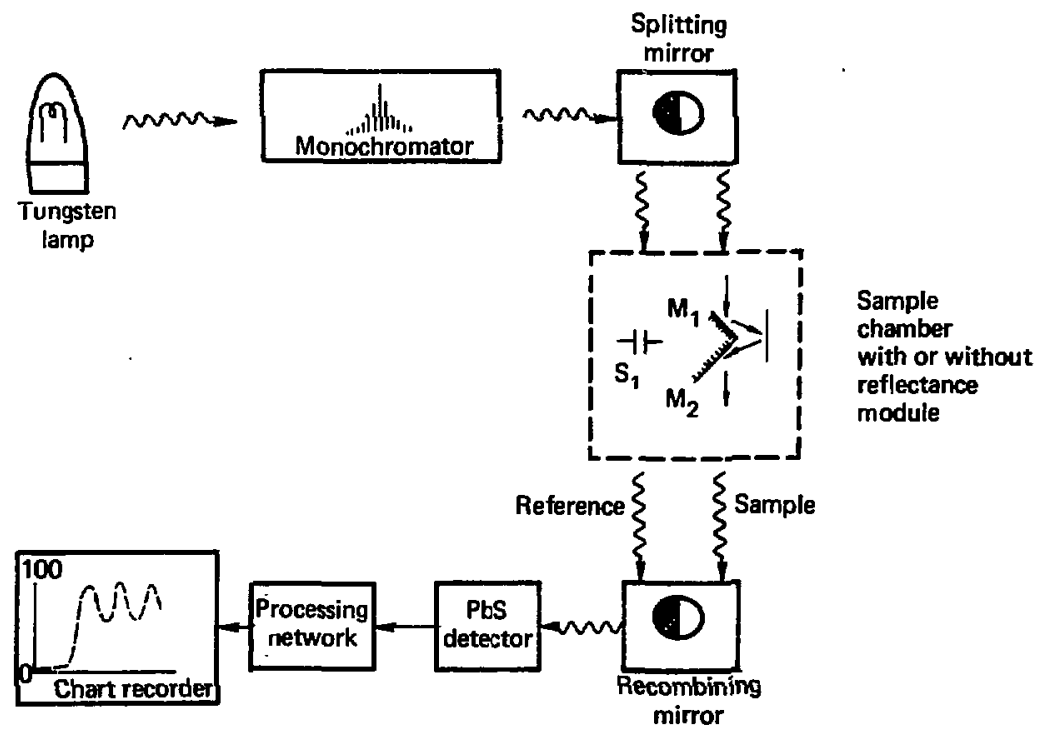

Figure 23. Function diagram of spectrophotometer. 
Enlarged view with dewar cap separated from dewar
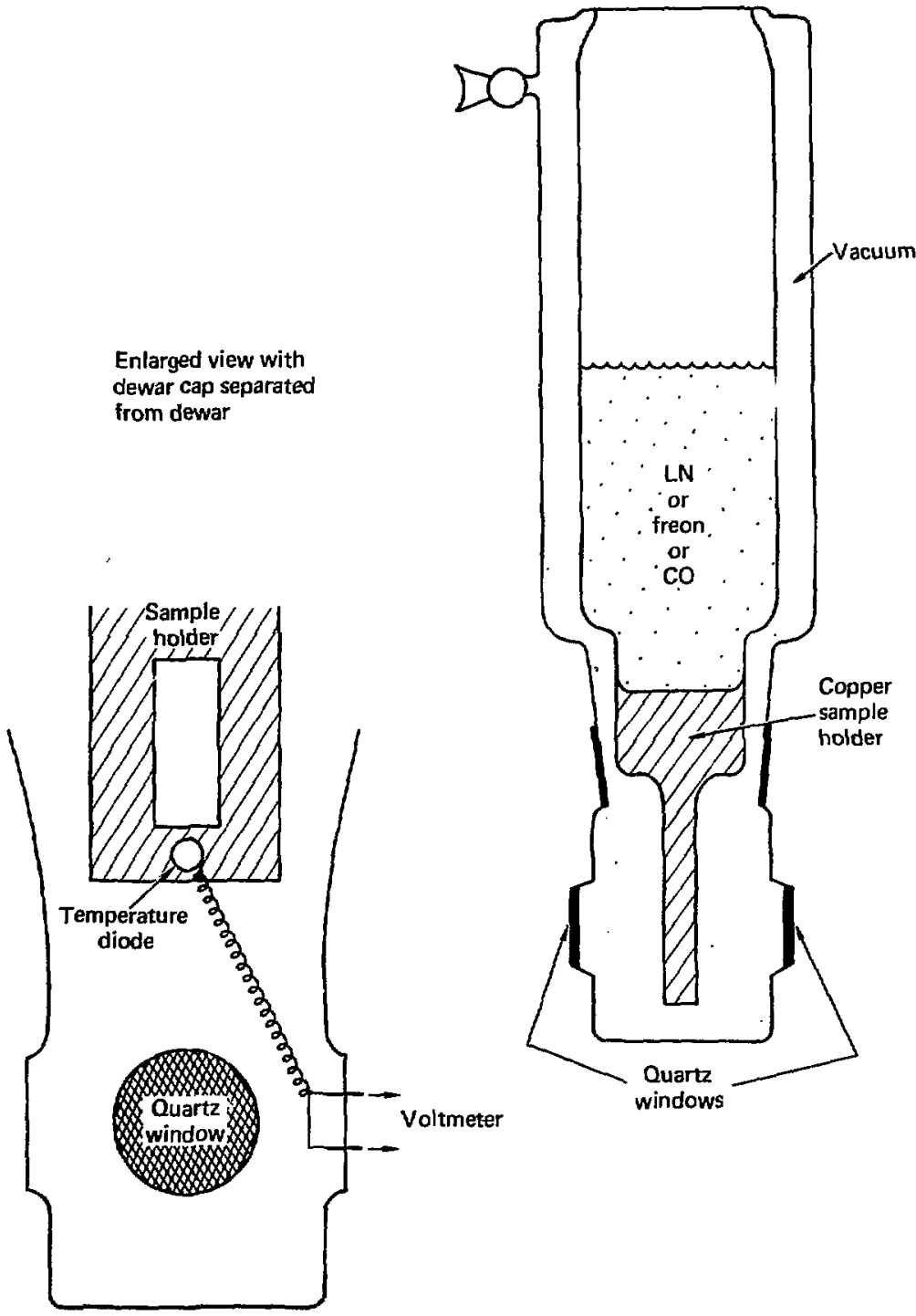

Figure 24. Tranemission dewar. 
windows are in line with the $1^{\prime \prime} \times 1^{\prime \prime}$ sample and the $1^{\prime \prime} \times 3 / 8$ " slot. The reference beam had to be apertured to correct for the losses through the dewar. A calibrated $\mathrm{Si}$ diode was used to monitor the temperature. It is connected to Kovar feed-throughs by two $15 \mathrm{~cm} \times 5 \mathrm{mil}$ diameter insulated copper wire. The lead voltage was negligible compared to the diode voltage when a $10 \mu \mathrm{A} . \mathrm{c}$. current was supplied to the circuit. The original intent was to use three different refrigerants to obtain three onstant temperatures for making measurements. After evacuating the dewar with a roughing pump and liquid nitrogen cold trap the samples could be cooled down with liquid nitrogen $\left(77^{\circ} \mathrm{K}\right)$, dry ice $\left(194^{\circ} \mathrm{K}\right)$, and Freon-12 $\left(243^{\circ} \mathrm{K}\right)$. A fourth temperature was obtained by freezing Freon-12 with liquid nitrogen and allowing it to warm up its melting point $\left(115^{\circ} \mathrm{K}\right)$. In the course of doing the experiments it was found that even during a warming cycle the temperatures change was slow enough to make measurements at other more convenient temperatures while admitting no more than $\pm 2^{\circ} \mathrm{K}$ error for the duration of the measurements. 


\section{Data Reduction}

The optical property of any material is characterized by its complex index of refraction

$$
\mathbf{N}=\underline{\mathbf{n}}-\mathbf{i k}
$$

.here $\underline{n}$ is the simple index of refraction, and $\underline{k}$ is the extinction coefficient. The tormer is responsible for real phase shifts and the latter for intensity attenuation of an electromagnetic wave as it propagates through an optical medium. For a wave traveling in the positive $z$ direction, the phase shift is

$$
\varphi=4 \pi \underline{n z} / \lambda
$$

where $\lambda$ is the wavelength of the wave, and the attenuation goes as

$$
e^{-4 \pi k z / \lambda}=e^{-a z}
$$

where $\alpha \equiv 4 \pi \underline{k} / \lambda$ is the absorption coefficient. Both $\underline{n}$ and $\alpha$ are functions of energy. If one or both are known for all wavelengths the band structure of the material can be specified. If $a$ of $a$ semiconductor is known in the infrared range then some very important features of the band structure can be deduced (e.g., magnitude and type of bandgap).

Before one can attempt the inierpretation of the band structure the raw data must be reduced. Thin semiconductor films, and $\mathrm{Cu}_{x} \mathrm{~s}$ in particular, present a unique set of problems in this regard. In general, in order to solve the two unknown parts of $\mathrm{N}$ two independent sets of data are needed, usually $\underline{n}$ and $\alpha$ in the optical range of interest. In properly prepared bulk materials $\underline{n}$ and $\alpha$ are simply related to and $R$, but in thin $f i l m s$ the presence of interference 
effects complicates the solution considerably. Expressions relating and $R$ to $\alpha$ and $\underline{n}$ of an absorbing $f i l m$ on a transparent substrate were derived by Heavens. [118] But in his derivations he made the approximation that the thick transparent substrate can be considered as the final optical element, neglecting the surrounding air and the final substrate/air interface. Apparently this was based on the fact that the air is transparent and that reflective losses due to the substrate/air interface is negligible $(=4 \%)$ compared to the absorbance of the film. But the approximation has a profound effect on the interference characteristics. Since the interference patterns are due to multiple reflections from the various interfaces, all of which make comparable contributions, neglecting one contribution will result in misinterpretation of the fine structures in the data. These effects appear most strongly in the low $\alpha$ domain where important information such as the structure of the band edge and mechanisms involved in free carrier absorption may be lost if the data is not properiy analyzed. A derivation based on these considerations is given in Appendix $I$. The resuiting expressions for $T$ and $R$ are presented here for discussion.

$$
\begin{aligned}
& T=\frac{Z}{A_{1} e^{a d}+B_{1} \cos \varphi+C_{1} \cos \varphi+D_{1} e^{-a d}} \\
& R=\frac{A_{2} e^{a d}+B_{2} \cos \varphi+C_{2} \cos \varphi+D_{2} e^{-\alpha d}}{A_{1} e^{a d}+B_{1} \cos \varphi+C_{1} \cos \varphi+D_{1} e^{-a d}}
\end{aligned}
$$

where $A_{1}, A_{2}, B_{1}, B_{2}, C_{1}, C_{2}, D_{1}, D_{2}$ and $z$ are functions of the real and imaginary parts of the complex indices of refraction of the various media, and the film thickness. In general, $\left|B_{1}\right| \gg C_{1}$, so the interference effects are predoninantly represented in the $B_{1} \cos \varphi$ term.

Theoretically, $\underline{n}$ and a can be solved either graphically or numerically if $T, R$, and $d$ are known. But in practice the solutions are 
either nonexistent or multi-valued. Denton et al [119] showed that small errors in $d(<5 \%)$ and $T$ and $R(<1 \%)$ are sufficient to invalidate the use of the above relations. Moreover, in their demonstration calculations they assumed a constant index of refraction

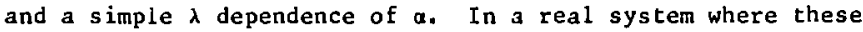
niceties seldom exist the sensitivity to experimental error is even greater. Even if a solution is obtained it may not be the correct one. Erroneous $T$ and $R$ data may lead to a solution if a compensating error is allowed in d. Finally, there are many cases where there are no solutions at all, partly because Eqs. (4-11) and (4-12) do not take into consideration certain realities like surface non-uniformity and internal porosity in the films.

The method used to reduce the optical data in the present work allows one to obtain a solution without introducing artifacts. The error is at worst a linear sum of the experimental uncertainties. Instead of solving for $\underline{\underline{n}}$ and $\alpha$ simultaneously with coupled equation, the two quantities are computed independently with relatively simple formulas. This approach avoids the avalanching effects of carrying over small errors in successive approximation techniques. Though it lacks the theoretical precision of a more complicated approach, it allows one to obtain a meaningful solution from real data.

From Eq. (4-11) one can see that T oscillates with $p$, especially when $\alpha$ is small. Appendix I shows that $T$ is proportional to $\left(A_{1}-\left|B_{1}\right| \cos \phi\right)^{-1}$ where $A_{1}>\left|B_{1}\right|$ and $B_{1}<0$. It is a maximum when

$$
\varphi=4 \pi n_{M} d / \lambda_{M}=Q(2 \pi)
$$

and a minimum when

$$
\varphi=4 \pi n_{m} d / \lambda_{m}=(Q \pm 1 / 2)(2 \pi)
$$


where $Q$ is an integer,

$\lambda_{M}$ and $\lambda_{m}$ are successive interference maximum and minimum wavelengths, respectively,

"+ is for $\lambda_{m}<\lambda_{M} \quad-"$ for $\lambda_{m}>\lambda_{M}$.

In the far infrared where $\underline{n}$ is relatively constant Eqs. (4-13) and (4-14) can be combined to give

$$
Q=\frac{\lambda_{m}}{2\left|\lambda_{M}-\lambda_{m}\right|}
$$

With $Q$ computed and rounded to nearest integer, $\underline{n}$ at all the extrema can be computed from

$$
\mathbf{n}=Q \frac{\lambda_{M}}{2 \bar{d}}
$$

or

$$
n=\left(Q \pm \frac{1}{2}\right) \frac{\lambda m}{2 d}
$$

with $Q$ increasing with shorter wavelengths and the conditions for "+" or "-" the same as those in Eq. $(4-14)$. The computed values of $\underline{n}$ are fitted by least mean squared to a quadratic function of $\lambda$, the simplest function which will allow for curvature in the dispersion relationship. The error in $\underline{n}$ is 1 imited to uncertainties in specifying the extrema wavelength and in measuring $d$. With reasonably accurate $\lambda_{M}$ and $\lambda_{m}$ (about $95 \%$ accuracy) $t$ s s computed $Q$ can easily be rounded off to its true integer value. The self-correcting feature of this procedure reduces much of the initial errors. If $\underline{n}$ was computed irectly from

$$
n=\frac{\lambda_{m} \lambda_{M}}{2 \mathrm{dI} \lambda_{M}-\lambda_{m}{ }^{\prime}}
$$


$5 \%$ errors in $\lambda_{M}, \lambda_{m}$, and $d$ can result in as much as $20 \%$ error in $n$. But by using the procedure outlined above ote can limit the starting error to le 33 than $15 \%$ in $Q$ which can then be reduced to its exact value by rounding to nearest integer.

It is best to start at the longest wavelengchs where 1 ) the assumption thet $\underline{n}$ is constant is iust valid; 2) $\lambda_{M}-\lambda_{m}$ is largest and therefore least susceptible to uncertainties in $\lambda_{M}$ and $\lambda_{m}$; and 3 ) computed $Q$ is smallest and is least likely to be rounded off to wrong integer.

once $\underline{n}(\lambda)$ is determined, $a(\lambda)$ can be computed from tine transmittance equation by a simple iteration scheme. But instead of using Eq. (4-11) it is more appropriate and simpler to use a statistically averaged transmittarce, $\langle T\rangle$, and making the film thick enough $(>0.7 \mu \mathrm{m})$ to reduce possible error due to interference. The rationale of this approach is outlined in Appendix $I$. The expression used in this iteration process has the form

$$
a=d^{-1} \text { en } \frac{Z /\langle T\rangle}{\left[\left(A_{1}+D_{1} e^{-2 a d}\right)^{2}-B_{1}^{2} e^{-2 a d}\right] 1 / 2}
$$

In the long wavelength region where the interference patterns are very obvious, only those data points which correspond to mid-values in the oscillations were analyzed.

The value of $\underline{n}(\lambda)$ and $a(\lambda)$ can be computed from the $T$ data alone if the interference structure is resolvable. This approach is well suited for polycrystalline materials with non-spacular surfaces. Scattering losses will reduce the magnitude of the measured reflectance but the interference patterns should still be distinguishable in $T$ and $R$. The $R$ spectrum is usually more desirable for determining $\lambda_{M}$ and $\lambda_{m}$ because the interference pattern in $R$ is less susceptible to distortions caused by changes in $a$. 


\section{Electrical Measurements}

\section{Instrumentation}

The resistivity and $\mathrm{Ha}:$ effects in $\mathrm{Cu}_{x} \mathrm{~S}$ films were measured at temperatures ranging from liquid nitrogen to room temperatures. The sample dewar was supplied by Janis Research. The sample temperature was controlled with a Lakeshore Cryotronic TGC-100 controller. A calibrated GaAs diode, embedded in the copper sample holder, was used to monitor the temperature and several turns of nichrome wire wrapped around a cyclindrical sample cover served as a heater. The temperature could be controlled to within $1^{\circ} \mathrm{K}$.

A sample is initially cooled overnight by radiation from a liquid nitrogen reservoir. Temperatures of about $90^{\circ} \mathrm{K}$ can be achieved by this means. The slow cooling process was necessary to minimize the possibility of contacts popping of from the sample, usually with part of the film still attached to the leads. Any sudden changes in the temperature ( $>10^{\circ} \mathrm{K} / \mathrm{min}$ ) during cooling or heating could result in the destruction of the thin film sampie. The final cooling down to $77^{\circ} \mathrm{K}$ was achieved by opening a needle valve which separated the liquid nitrogen reservoir from the inner sample chamber and allowing a cold vapor to be sprayed onto the sample. Lower temperatures could be achieved by pumping on the sample chamber but the resulting turbulence was detrimental to stable electric measurements.

The samples were prepared in a van der Pauw configuration. [120] Four A11 contact pads, 1/16" diameter and 3000 A thick, were evaporated onto the corners of the $1 \mathrm{~cm} \times 1 \mathrm{~cm}$ samples. The Au pads provided ohmic contacts for the purpose of measuring the small Hall voliages. Without the Au pads the thin oxide surface layer on the $\mathrm{Cu}_{x} \mathrm{~s}$ was cufficiently non-ohmic to cause unstable Hall voltage measurements. Normal resistivity measurements were not sensitive to the oxide layer. Coated "38" gauge copper leads were attached to the pads with an Ag doped 
ep.oxy. A silvered paint was tried in place of the epoxy but the contacts tend to slide off during the initial cooling due to the ditference in expansion coefficients. The strength and integrity of the epoxied contacts were too of ten demonstrated when contacts were torn off during a fast cooling cycle.

The electrical measurements were made with a Leeds-Northrup "2760" digital voit :eter. A d.c. curtent ranging from 50 to 500 uA was supplied to the sample by a Keithley "225" current source. All leads connecting the various electrical components to the inlet feedthroughs of sample dewar were shielded coaxial wires. The dewar itself was entirely stainless steel. A reversible magnetic field of $8.6 \mathrm{k}$ gauss was provided by a Varian V-4005 four inch electromagnet. A 60 amp magnetizing current was supplied by a Varian V-2901 regulated power. supply. The magnet and power s'sply were both water cooled.

Ine system was capable of resolving a Hall voltage of $1 \mu v$. The major cause of instability was resistive heating in the thin sample by tne sample current. To minimize thermal transients the sample current was chosen as small as possible, yet, allowing for the greatest number of significant digits in the data. At low temperatures $\left(<100^{\circ} \mathrm{K}\right)$, where the thermal instabilities are greatest, the sample current was left on between measurements so that the sample was always at a near equilibrium state. Room temperature measurements were taken at the beginning and end of a run to check on the ef ect of the current and thermal cycling on the filins. No discennible difference was observed.

A functional schematic of the measuring system and a sketsh of th sample iolder is presented in Figs. 25 and 26, respectively. 


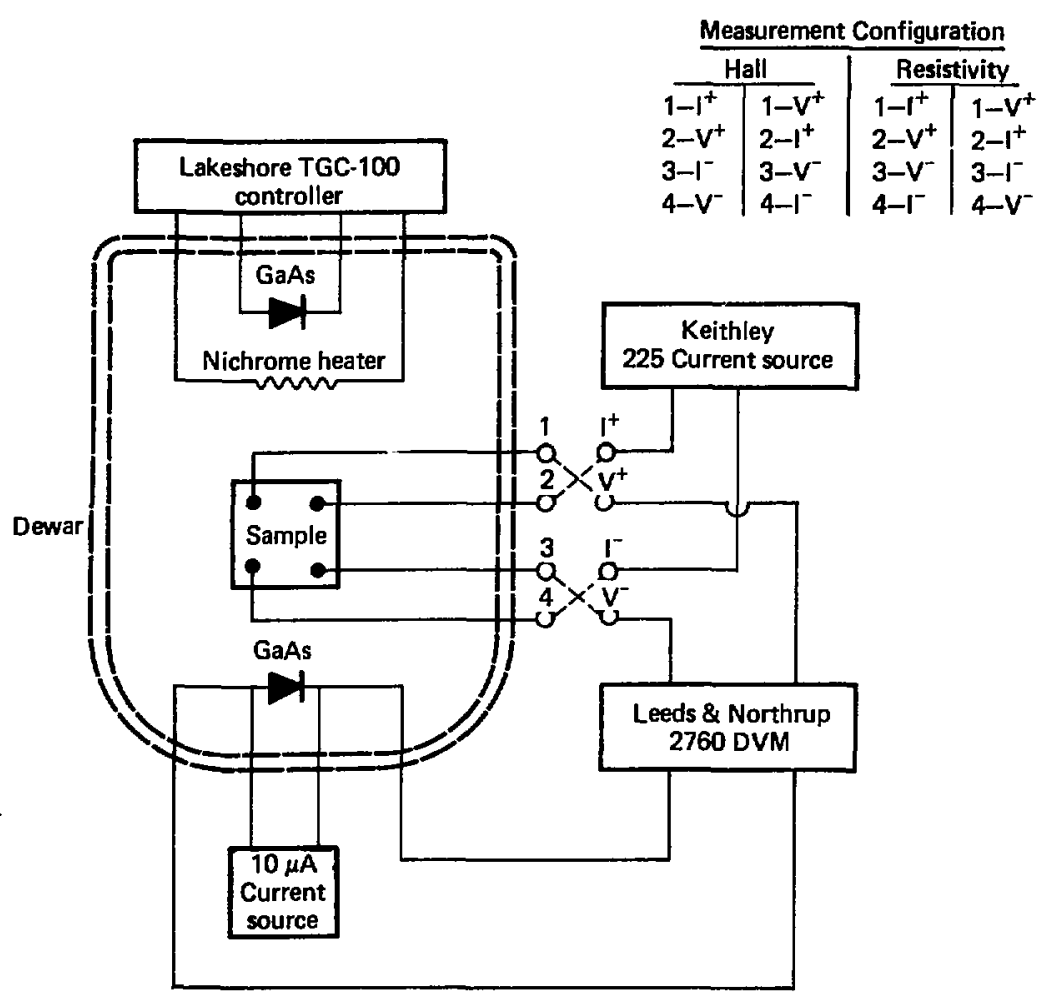

Figure 25. Functional diagram of Hall effect system. 


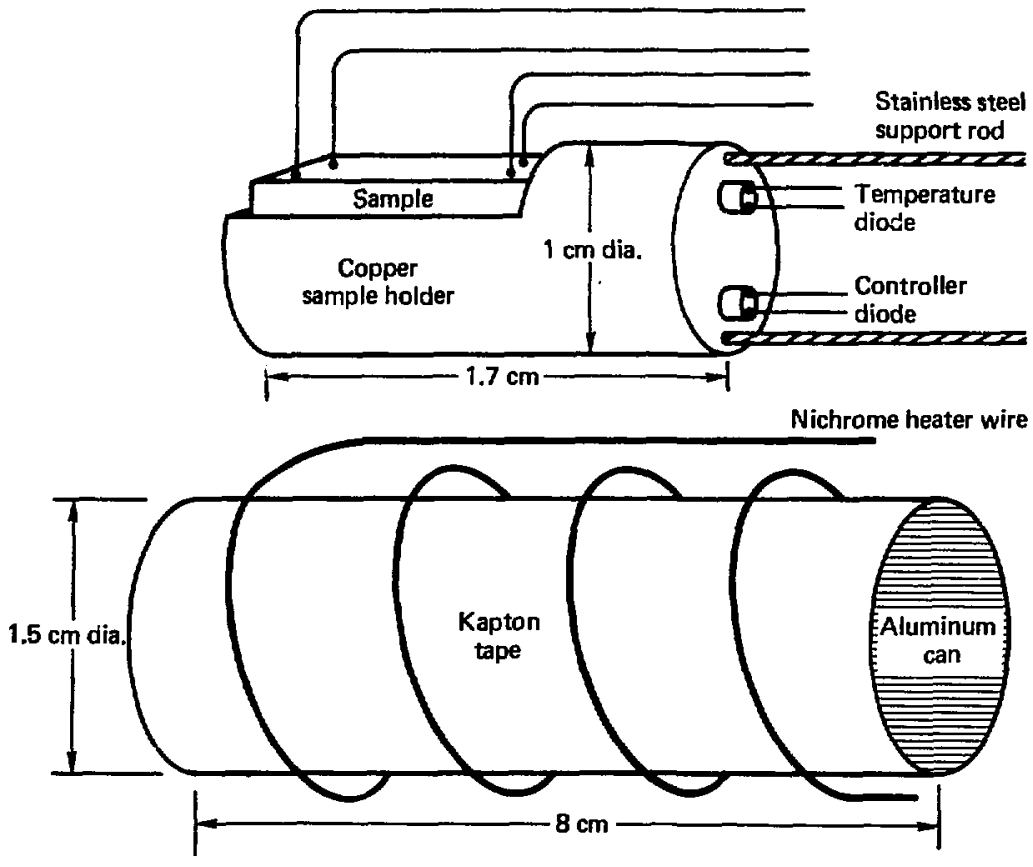

Figure 26. Hall sample holder and heater. 


\section{The Hall Effect}

Holes traveling with a velocity $\vec{v}$ in a semiconductor in the presence of a magentic fielu $\vec{B}$ will experience a Lorentz force

$$
\overrightarrow{\mathrm{F}}_{\mathrm{B}}=\mathrm{q}(\overrightarrow{\mathrm{B}} \times \mathbf{+})
$$

The polarization caused by the deflection of the mobile charges to one side and an ionized depletion layer on the opposite side of the semiconductor results in an electric field which will counterbalance the effects of the magnetic field.

$$
\overrightarrow{\mathrm{F}}_{\mathscr{B}}=\mathrm{q} \overrightarrow{\mathscr{E}}=-\overrightarrow{\mathrm{F}}_{\mathrm{B}}
$$

With the directions of the current, electric field, and magnetic Field defined according to Fig. 27a the resulting field is

$$
\begin{aligned}
\mathscr{E}_{y} & =v_{x}{ }^{B} \\
& =\frac{J_{x}}{p q} B_{z}
\end{aligned}
$$

where $J_{x}$ is the current density.

The Hall coefficient is defined by

$$
R_{H}=\frac{\mathscr{E}_{y}}{J_{x_{z}}}=+\frac{1}{p q},
$$

the sign changes from "+" to "-" for electrons. 


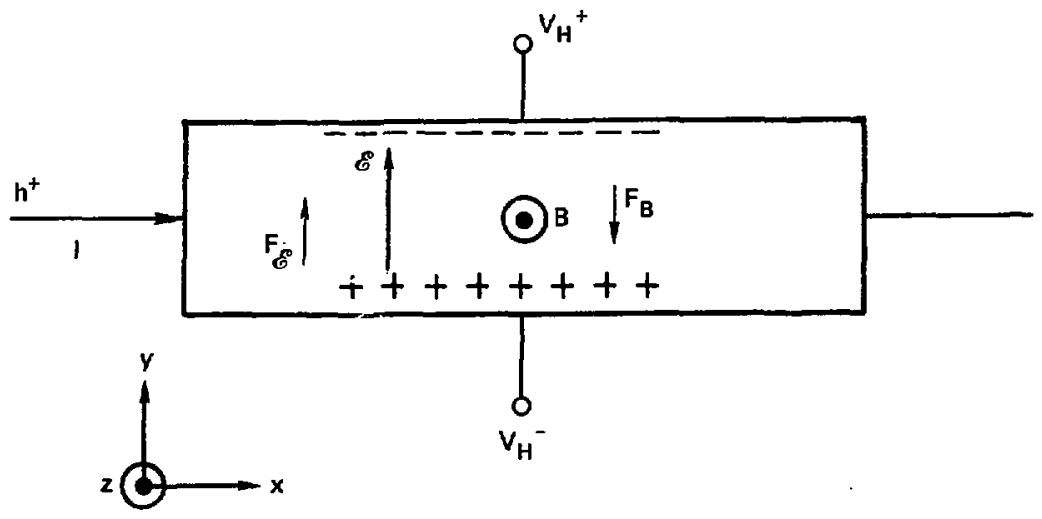

Figure 27a. Hall effect.

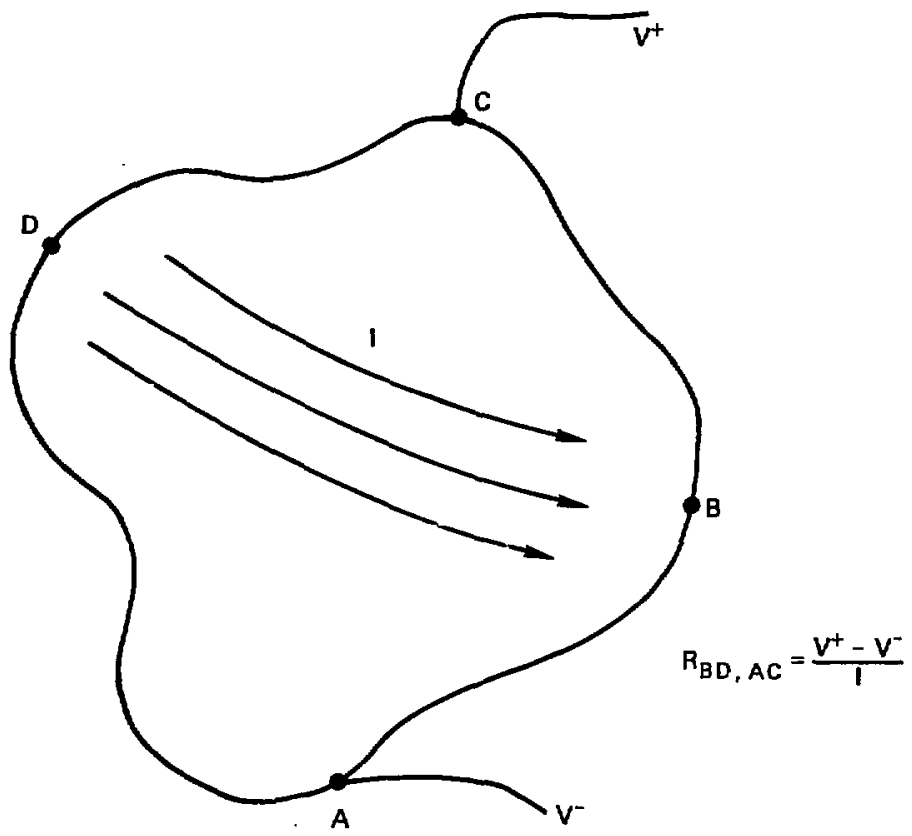

Figure 27b. Generalized van der Pauw configuration. 
The mobility is given by

$$
\begin{aligned}
\mu & =\frac{\sigma}{\mathrm{pq}} \\
& =\sigma \mathrm{R}_{H} .
\end{aligned}
$$

The simple expression in Eqs. (4-23) and (4-24b) assumes that the equal energy surfaces are concentric spheres centered at $\vec{k}=0$ and that all the carriers have the same velocity. More accurate analysis must take into account possible anistropy in the energy surfaces and the fact that the carriers are distributed according to Fermi-Dirac statitics. These two corrections enter the definition of $\mathrm{R}_{\mathrm{H}}$ through the anistropy ratio, $x$, and the scattering coefficient, $s,[121,122]$

$$
\mathrm{R}_{\mathrm{H}}=\frac{\mathrm{es}}{\mathrm{Pq}}
$$

The computation of requires detail knowledge of the band structure of a material. As it was pointed out in Chapter II, the band structure of $\mathrm{Cu}_{\mathrm{x}} \mathrm{S}$ is practically impossible to model accurately. To a first approximation the present work will assume that the energy surfaces near the band edge are isotropic, orc $=1$. The scattering coefficient is [123]

$S=\frac{\left\langle\tau^{2} /\left(1+\gamma^{2}\right)\right\rangle}{\left\langle\tau /\left(1^{3}+{ }^{2} \gamma\right)\right\rangle}\left\{\langle\tau\rangle+1_{c}^{2} \frac{\left\langle\tau^{2} /\left(1+\gamma^{2}\right)\right\rangle^{2}}{\left\langle\tau /\left(1+\gamma^{2}\right)\right\rangle}-\left\langle\frac{\tau^{3}}{1+\gamma^{2}}\right\rangle\right\}^{-1}(4-26)$

where $\tau$ is the scattering relaxation time

$$
\begin{aligned}
& \omega_{c}=\frac{q B}{{ }^{*}} \text { is the cyclotron resonance frequency } \\
& \gamma=\omega_{c} \tau
\end{aligned}
$$

and $\langle x\rangle \quad$ is the expectation value of the parameter $x$.

In the low field limit, $\gamma \ll 1$, in which the charge carriers traverse only a small fraction of the cyclotron orbit before they are scattered, so 


$$
S=\left\langle\tau^{2}\right\rangle \mid\langle\tau\rangle^{2} \text {. }
$$

In many cases $\tau$ is a simple power function of the energy

$$
\tau \sim \mathrm{E}^{\mathrm{P}}
$$

Then Eq. (4-27) gives $[122,124]$

$$
s=\frac{\Gamma(5 / 2) \Gamma(5 / 2+2 p)}{[\Gamma(5 / 2+p)]^{2}}
$$

Table 4 lists the scattering coefficients of four of the scattering mechanisms described in Chapter III-B. For polar optical phonon scattering a relaxation time cannot be rigorously defined. But Frolich and Mott ${ }^{[98]}$ have shown that a pseudo relaxation time can be defined which produces results that are very similar to more exact solutions. The relaxation time is not a simple power function of energy. Devin ${ }^{[125]}$ calculated the scattering coefficient due to polar optical phonon scattering. His results are reproduced in Fig. 28 .

The definition of mobility must be re-established with the above considerations. Eqs. (4-24a) and (4-24b) no longer defined the same quantity. Eq. (4-24a) defines the normal drift mobility, $\mu$, but Eq. (4-24b) is the definition of the Hall mobility. The scattering coefficient in terms of these two mobilities is

$$
S=\mu_{H} / \mu .
$$

From Table 3 and Fig. 27 one can see that $S \times 1$ with the exception of ionized impurities scattering and polar optical phonon scattering. The latter fluctuates very little, about 1.1 , so for comparative analysis this work make the simplifying assumption that all mechanisms have a constant sattering coefficient with that due to polar optical phonons set at 1.1 . 
TABLE 4. Scattering coefficients and energy dependence of common scattering mechanisms. [121]

\begin{tabular}{llcl}
\hline $\begin{array}{l}\text { Scattering } \\
\text { mechanism }\end{array}$ & $\tau(\mathrm{E})$ & $\mu(\mathrm{T})$ & $S$ \\
\hline Acoustic Phonon & $\tau \sim \mathrm{E}^{-1 / 2}$ & $\mu \sim \mathrm{T}^{-3 / 2}$ & 1.18 \\
Piezoelectric & $\tau \sim \mathrm{E}^{1 / 2}$ & $\mu \sim \mathrm{T}^{-1 / 2}$ & 1.10 \\
Ionized Impurities & $\tau \sim \mathrm{E}^{3 / 2}$ & $\mu \sim \mathrm{T}^{3 / 2}$ & 1.93 \\
Neutral Impurities & $\tau \sim \mathrm{E}^{\circ}$ & $\mu \sim \mathrm{T}^{\circ}$ & 1.00 \\
\hline
\end{tabular}




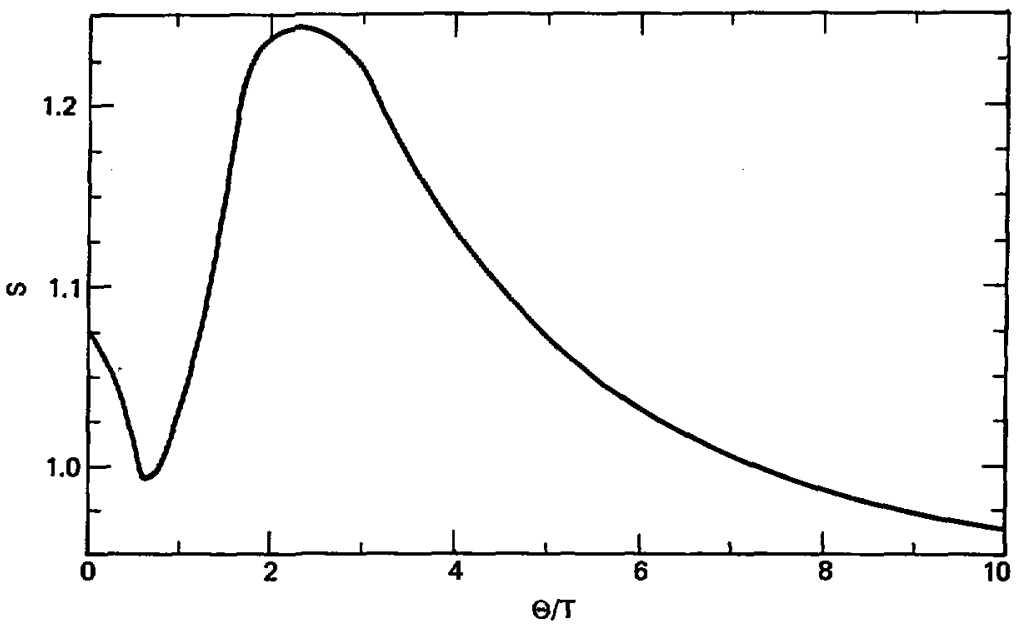

Figure 28. Scattering coefficient for polar optical phonon scattering [125]. 
The samples used in this study are in a van der Pauw configuration. A generalized configuration is shown in Fig. $27 \mathrm{~b}$. van der Pauw ${ }^{[120]}$ showed that it was possible to measure the resistivity and Hall effect in a flat sample of an arbitrary shape. The resistivity of the sample of Fig. $27 \mathrm{~b}$ would be

$$
\rho=\frac{\pi d}{l n 2} \frac{\left({ }_{A B, C D}+R_{B C, D A}\right)}{2} \pm\left(\frac{R_{A B, C D}}{R_{B C, D A}}\right)
$$

where $R_{A B, C D}$ is the resistance between points $C$ and $D$ with current conducting through points $A$ and $B$.

d is the sample thickness

and $\quad\left(\frac{R_{A B, C D}}{R_{B C, D A}}\right)$ is a function of the ratios of the two resistances.

The Hall mobility is given by

$$
H_{H}=\frac{d}{B} \frac{\Delta R_{B D, A C}}{\rho}
$$

where $\Delta R_{B D, A C}$ is the change in resistance $R_{B D, A C}$ due to the presence of the magnetic field.

The function $f=1$ when $R_{A B, C D}=R_{B C, D A}$. So if the material has uniform resistivity and if the four contacts are equally spaced, Eq. $(4-31)$ reduces to

$$
\rho=2.27 \mathrm{~d}\left(\mathrm{R}_{\mathrm{AB}, \mathrm{CD}}+\mathrm{R}_{\mathrm{BC}, \mathrm{DA}}\right)
$$

Ideally, the contacts should be point contacts located on the perimeter of the sample. The errors arising from the finite size of real contacts or from being located off the perimeter is 


$$
\begin{aligned}
& \frac{\Delta \rho}{\rho} \sim\left(\frac{\delta}{D}\right)^{2} \\
& \frac{\Delta \mu_{H}}{H_{H}}-\left(\frac{\delta}{D}\right)
\end{aligned}
$$

where $\delta$ is either the diameter of the contacts or the distance from the perimeter

and $D$ is the diameter of the sample.

With the four $1 / 16-$ inch diameter contacts on the $1 \mathrm{~cm}^{2}$ Hail samples, the estimated errors are

$$
\frac{\Delta p}{p} \times .02
$$

and

$$
\frac{\Delta \mu_{H}}{\mu_{H}} \simeq .10
$$

The moderate accuracy in the mobility measurements allows a reasonable quantitative interpretation of the data and at the same time justifies many of the assumptions and approximations made with regards to the analytic expressions. 


\section{Experimental Results and Discussion}

The experimental results are grouped into three categories. First, the effects of sputtering gas pressures on the optical and electrical properties of $\mathrm{Cu}_{x} \mathrm{~s}$ are presented and discussed. Second, the effects of two common heat treatments used in cell processing are explored and compared with the effects of sputtering gas pressures. Third, the band structure of $\mathrm{Cu}_{x} \mathrm{~S}$ is probed by studying the changes in optical absorption characteristics as a function of temperature.

In all these experiments it was not possible to ascertain the composition of the $\mathrm{Cu}_{x} \mathrm{~s}$ precisely. Microprobe analysis was tried but its sensitivity to the $\mathrm{Cu} / \mathrm{S}$ ratio was too gross. It could not distinguish between $\mathrm{Cu}_{2 .} \mathrm{S}$ and a more degenerate phase mixed with small amounts of free copper precipitates. $x$-ray diffraction only confirmed the presence of a phase but not necessarily its absence because the appearance of the three diagnostic peaks, two for chalcocite and one for djurleite, [19] is a function of the orientation of the film as well as its actual composition. So, its value for comparing films prepared in different runs is somewhat 1 imited. It was useful for monitoring phase changes in heat treatment studies where films from the same sputtering run and subjected to controlled post deposition heat treatments were compared.

The optical transmittance characteristics of a film was a fast and highly reliable way to determine the quality of a film in terms of its chalcocite content. The absorption spectrum can be compared with known single crystal spectra. $[23,126]$ The Hall effect and resistivity in the same or similar films were studied in order to obtain a correlation between the electrical and optical properties. 


\section{A. Effects of Sputtering Gas Pressure}

Preliminary studies $[28,111]$ indicated that the pressure of the sputtering gas mixture played a critical role in determining the properties of reactively sputterad $\mathrm{Cu}_{\mathrm{x}} \mathrm{s}$ films and $\mathrm{Cu} / \mathrm{CdS}$ solar cells. The observed differences in resistivity and optical absorbance was attributed to the differences in partial pressure of $\mathrm{H}_{2} \mathrm{~S}$ which was believed to be the main determinant of the chemical composition. The reflectance and transmittance data of films sputtered at different gas pressures are presented. The data is analyzed to determine the effects of gas pressure on the absorption characteristics, especially near the band edge. Corresponding hall effects data is analyzed to study the effects on free carrier concentration and mobility. The correlation of the two sets of experiments is used to extract further information about comon parameters. The given pressures are the absolute pressures of the $7 \% \mathrm{H}_{2} \mathrm{~S} / \mathrm{Ar}$ mixture.

The transmittance and reflectanc spectra of a highly stoichiometric film (\#19A) is presented in Fig. 29. The 0.7 $\mu \mathrm{m} \mathrm{film}$ was sputtered in $15 \mu \mathrm{mg}$ of $\mathrm{H}_{2} \mathrm{~S} / \mathrm{Ar}$. The characteristic features of a highly stoichiometric film is demonstrated here. The sharp transition between the absorbing and transmitting regions are indicative of a sharp band edge with minimal band tailing. The nearly constant and high interference peaks are due to low free carrier concentration and absorption. One other interesting feature of this set of data is the small change in curvature of the transmittance spectra near $1.0 \mu \mathrm{m}$. From the reflectance data it can be seen that the deviation is due to an interference extrema. For thinner filme the extrema are broader and more prominent. The transmittance at the extrema wavelength will take on a knee-like characteristic which can easily be misinterpreted to be due to real band structures. Measurements performed on $\mathrm{Cu}_{x} \mathrm{~s}$ films which are part of solar cells are susceptible to this misinterpretation because the thicknesses are less than $0.5 \mu \mathrm{m}$. 

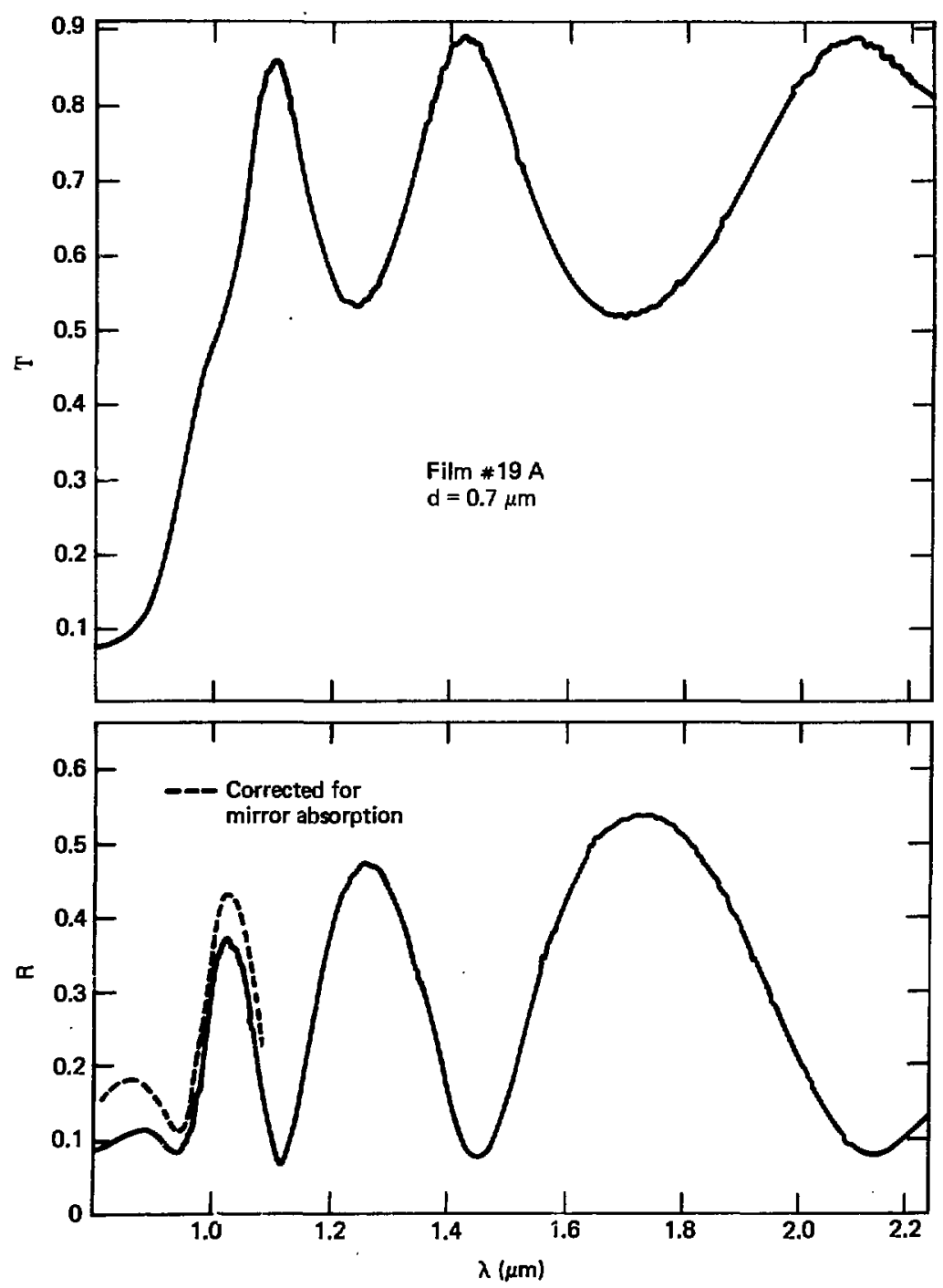

Figure 29. Transmittance and reflectance spectra of near stoichiometry $\mathrm{Cu}_{x} \mathrm{~S}$ film. 
The absorption coefficient and index of refraction derived from the transmittance and reflectance data of sample \#19A are shown in Fig. 30. The results compare well with that of single crystal $\mathrm{Cu}_{2.0} \mathrm{~S}$ with 1 ight polarized perpendicular to the c-axis. [23] To test whether the bandgap is direct or indirect, $\alpha^{2}$ and $\alpha^{1 / 2}$ are plotted against the photon energy in Fig. 31. From Eq. (3-5) one can see that the $\alpha^{1 / 2}$ versus hv plot should be a straight 1 ine over the range of energies for which the transitions are indirect. The indirect gap can be identified by extrapolating to $a^{1 / 2}=0$. The extrapolated intercept with the energy axis is actually $E_{g} \pm k_{B} \theta$, but the phonon energy $\left(k_{B} \theta\right)$ is much less than the bandgap energy. According to Eq. (3-1), the $\alpha^{2}$ versus hv should be linear over the range of energies for which the transitions are direct. The direct gap can be determined by extrapolating to $\alpha^{2}=0$, but this assumes that no other types of transition are taking place near the direct band edge. But, Fig. 31 shows that indirect transitions make significant contributions to $\alpha$ at low values of $\alpha^{2}$. An alternate convention for identifying the direct gap is to assign it to the transition point at which the $\alpha^{2}$ versus hv plot begins to have a "direct" characteristic. The underlying assumption is that the probability of the non-direct transition saturates at that point. This approach tends to over estimate while the extrapolated intercept tend to under estimate the direct gap. The difference between the two estimates is less than $0.03 \mathrm{eV}$. This amount of uncertainty is or the same order as the uncertainty in the raw data ( $\Delta \lambda \geq .02$ um translates into $\Delta E=.02 \mathrm{eV}$ ). The latter convention was chosen for the present analysis because the underlying assumption is more reasonable and it is less subject to changes in the slopes of the $\alpha^{2}$ versus hv plot. The $\alpha^{1 / 2}$ versus $h v$ plot identifies an indirect bandgap near $1.16 \mathrm{eV}$ and the $a^{2}$ versus $\mathrm{hv}$ plot identifies a direct gap at $1.31 \mathrm{eV}$. Either the hole or electron effective mass can be determined from the shape of the $a^{2}$ versus $h v p l o t$ if the other effective mass is known. 
-96-

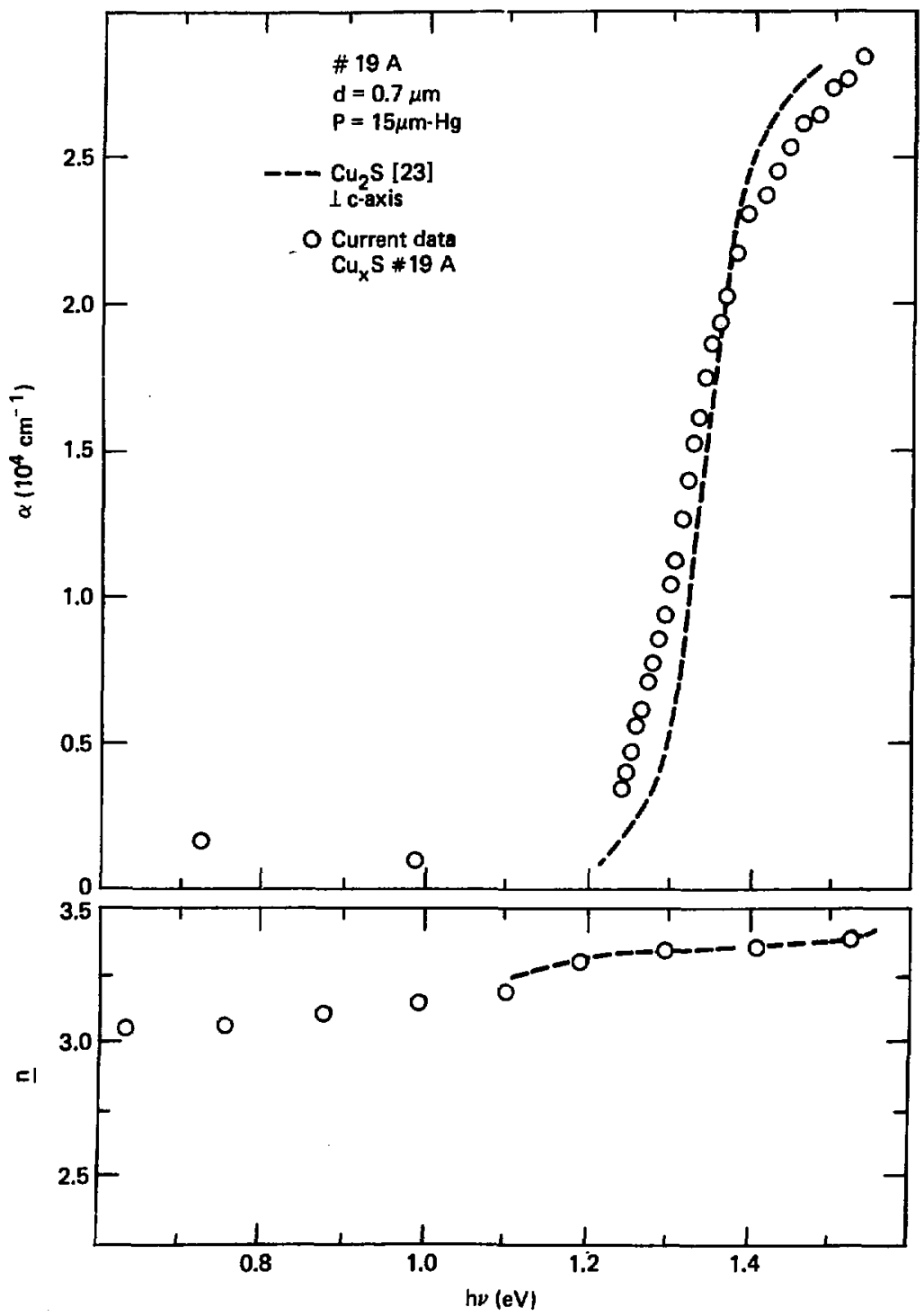

Figure 30. Absorption coefficient and index of refraction of high stoichiometry $\mathrm{Cu}_{x} \mathrm{~S}$ film. 


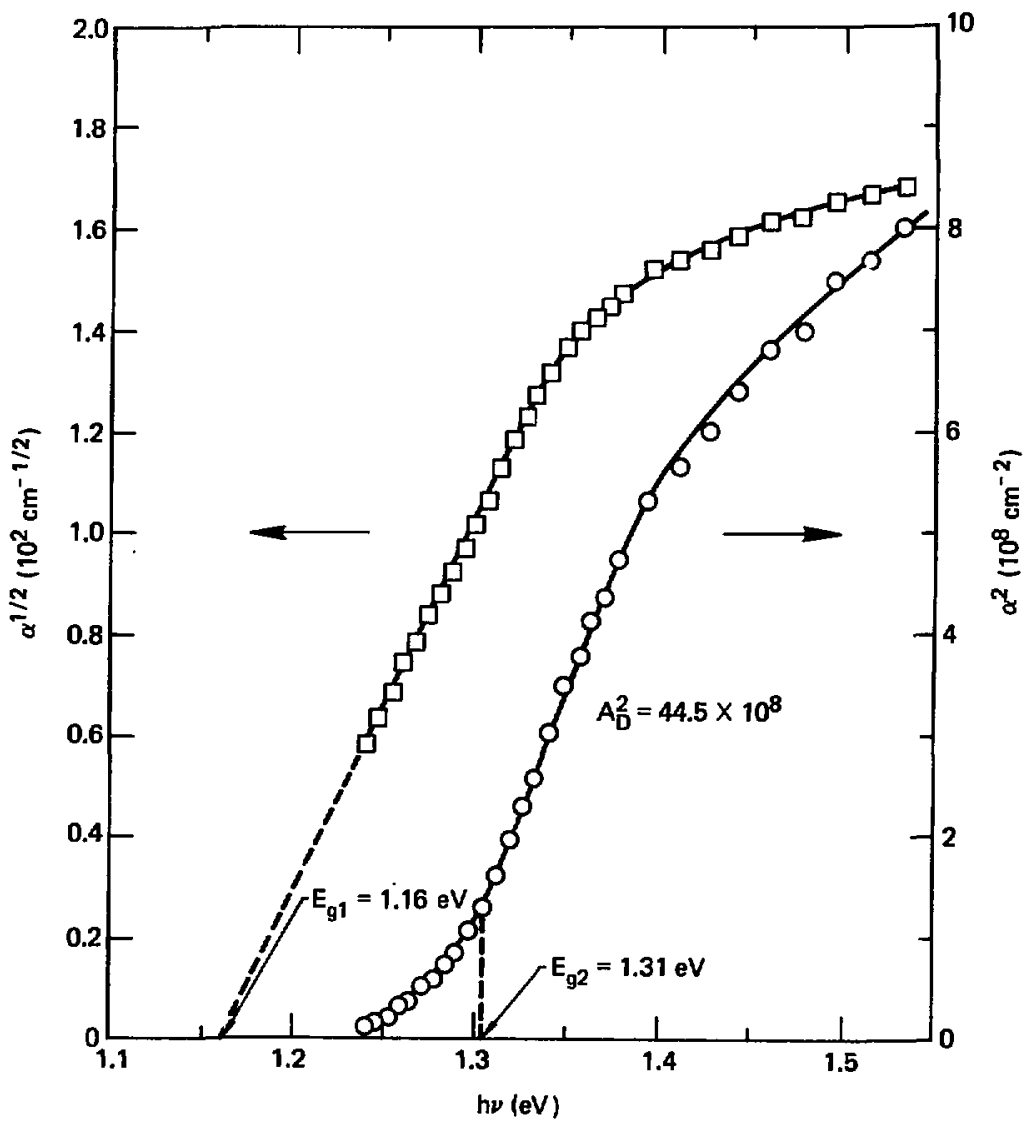

Figure 31. Band Gap Analysis of high stoichiometry $\mathrm{Cu}_{x} \mathrm{~S}$ film. 
From Eq. (3-1)

$$
\frac{\mu}{m_{o}}=\frac{1}{2}\left[\frac{\left(\pi A_{D} \underline{n} a_{o}\right)^{2} E_{o}}{\left(1+\frac{m_{o}}{m_{h}^{*}}\right)^{2}}\right]^{1 / 3}
$$

where $A_{D}^{2}$ is the slope.

With $A_{D}^{2}=44.5 \times 10^{8} \frac{\mathrm{cm}^{-2}}{\mathrm{eV}}$

$$
\underline{\mathrm{n}}^{2}=10 \text { and } \mathrm{m}_{\mathrm{h}}^{*} \approx 1.7 \mathrm{~m}_{0}^{[127]} \text { Eq. (4-1) gives } \mathrm{m}_{\mathrm{e}}^{*}=1.1 \mathrm{~m}_{\mathrm{o}}
$$

A plot of $\log (\alpha)$ versus $h v$ is showi in Fig. 32.

The nearly linear region for $E \simeq 1.3 \mathrm{eV}$ suggests the possibility of an Urbach effect. The slope of the linear region gives a characteristic temperature of $653^{\circ} \mathrm{K}$. Based on observations in other materials ${ }^{[62-65]}$ one would have expected a characteristic temperature near room temperature.

Resistivity $(p)$, hole concentration $(p)$, and Hall mobility $\left(u_{H}\right)$ of a similar film (\#19B) prepared in the same sputter run is presented in Figs. 33 and 34. Theoretically, the energy levels of various localized states can be determined from the slopes of the $\log p$ versus $1 / \mathrm{T}$ curve in Fig. 33. But the concentration of 10 alized states is so high that impurity banding has made it impossible to identify individual levels. It's uncertain whether the continuously changing slope is due to many very close lying states or is simply the transitions region between two impurity bands. If it is the latter then from. 


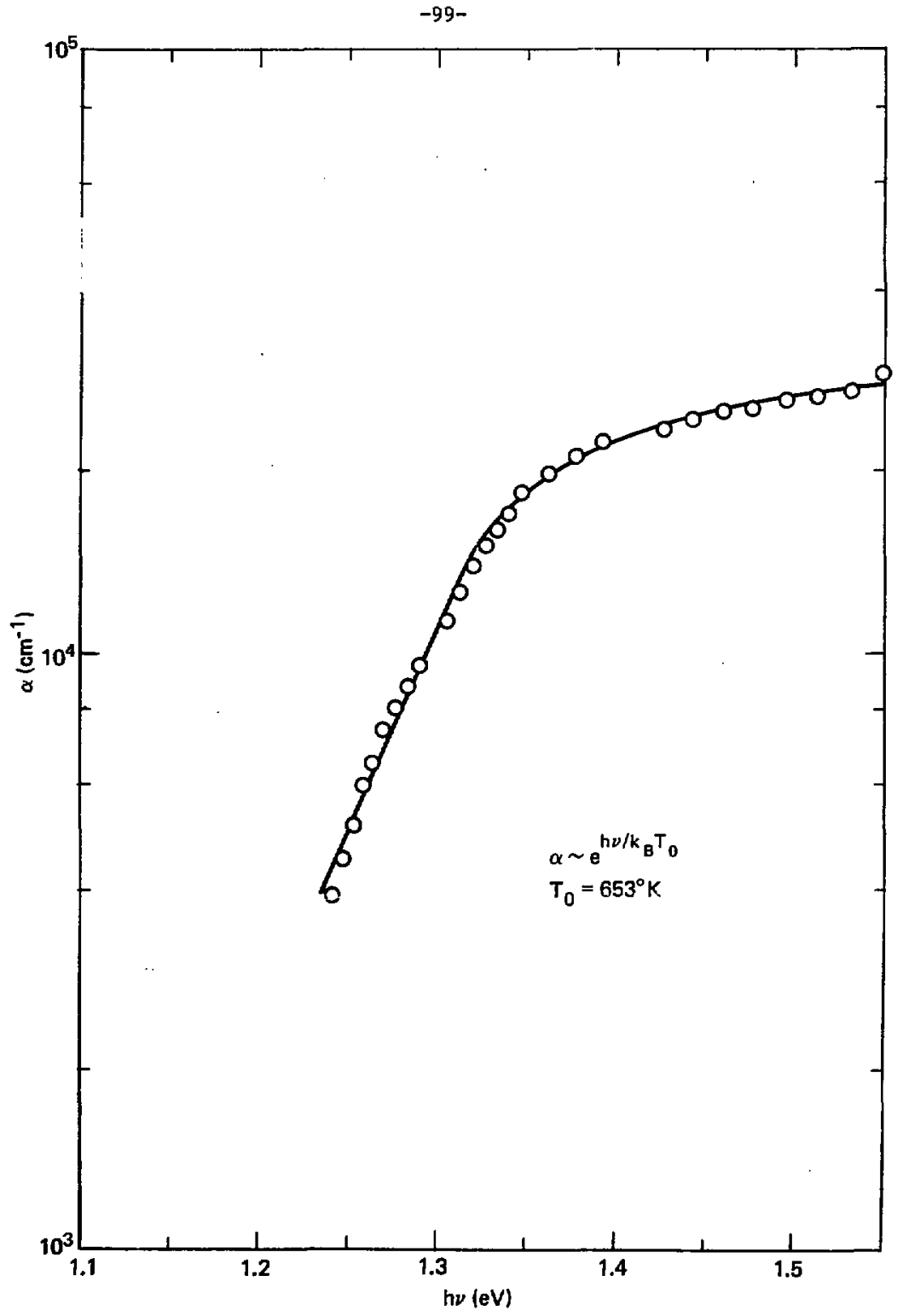

Figure 32. Urbach effect in high stoichiometry $\mathrm{Cu}_{x} \mathrm{~S}$ film. 


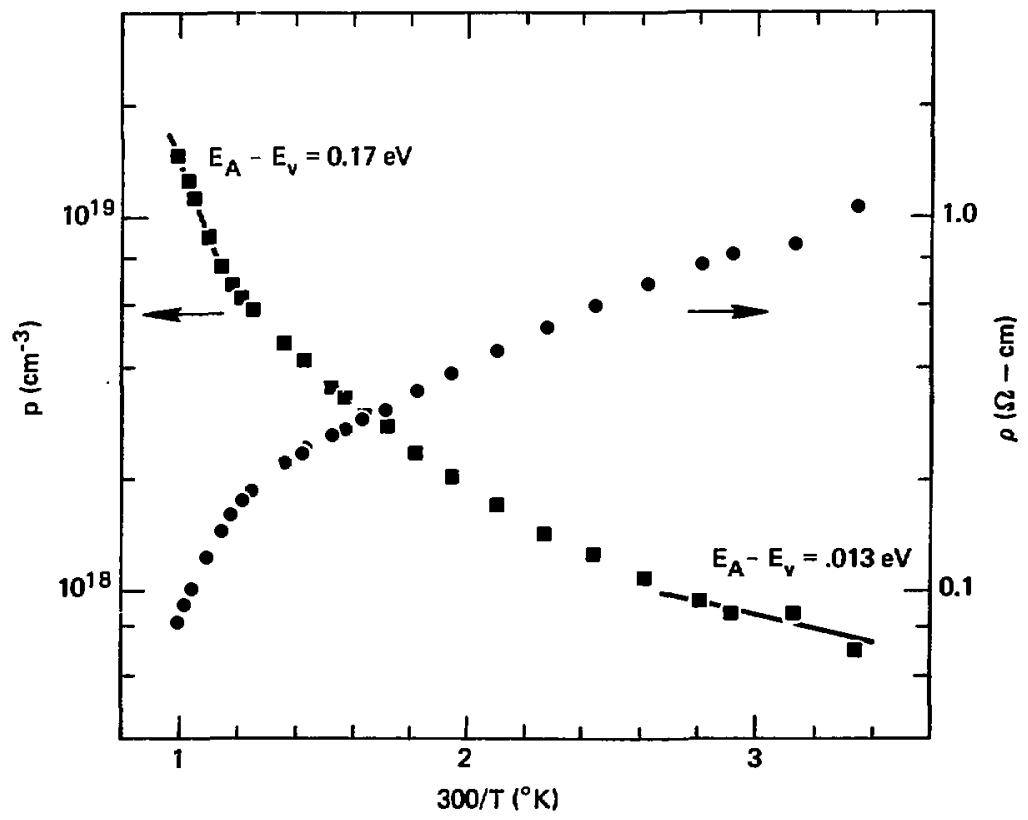

Figure 33. Resistivity and hole concentration of high stoichiometry $\mathrm{Cu}_{\mathrm{x}} \mathrm{S}$ films. 


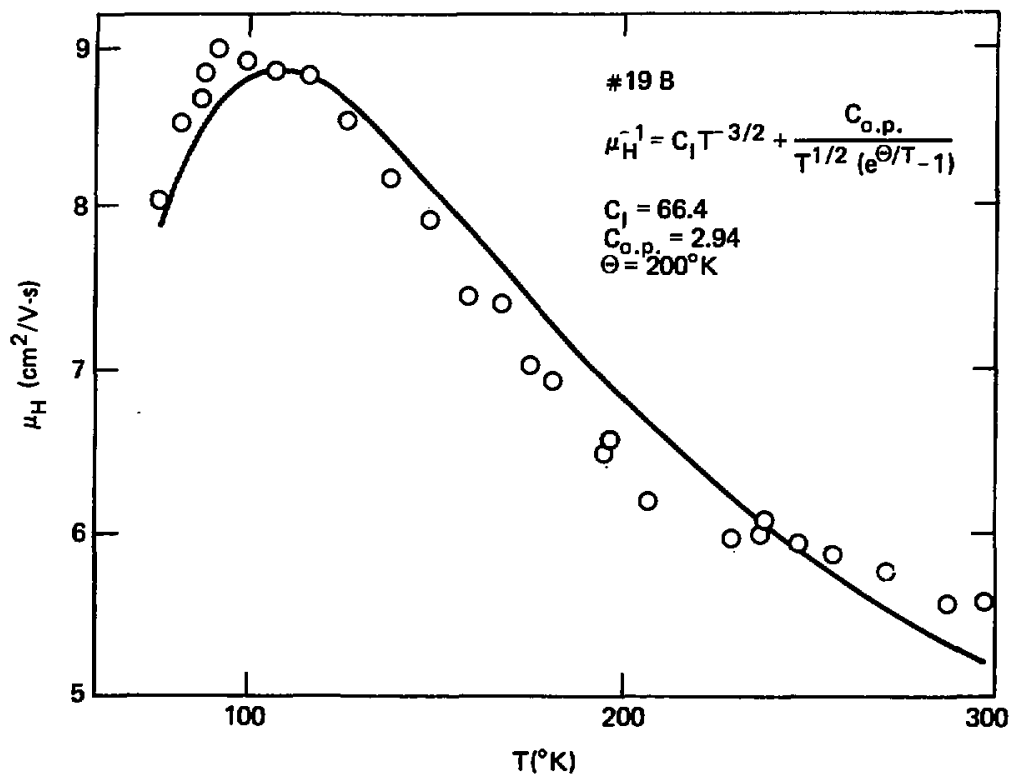

Figure 34. Hall mobility of high stoichiometry $\mathrm{Cu}_{x} \mathrm{~S}$ film. 


$$
P \sim e^{-\left(E_{v}-E_{A}\right) / 2 k_{B} T}
$$

where $E_{A}$ is the acceptor energy level,

two levels, one at $\left(E_{A 1}-E_{v}\right)=0.013 \mathrm{eV}$ and another at $\left(E_{A 2}=0.17 \mathrm{eV}\right)$, are estimated from the shallowest and steepest slopes, respectively. The fact that the banding extends down to 1 iquid nitrogen temperature suggests that valence band tailing in this p-type semiconductor may be taking place as a result of shallow acceptors levels merging with the valence band.

The Hall mobility in Fig. 34 was analyzed by assuming a maximum of five independent scattering mechanisms--ionized and neutral impurity scattering, nonpolar acoustic and polar optical scattering, and piezolectric effects. The best fit of the data indicates that the dominant scattering mechanisms are ionized impurity scattering at low cemperatures $\left(\mathrm{T}<100^{\circ} \mathrm{K}\right.$ ) and polar optical phonon scattering at high temperatures $\left(\mathrm{T}>150^{\circ} \mathrm{K}\right)$.

The transmittance and reflectance spectra of three other films are presented in $35 a$ and $b$, respectively. All are of comparable thickness. Film $\# 20 \mathrm{C}$ is $1.0 \mu \mathrm{m}$ thick, 非28 $\mathrm{A}$ is $1.05 \mu \mathrm{m}$ thick, and thick. They were sputtered in $\mathrm{H}_{2}$ S/Ar pressures of 10,20 , and $25 \mu \mathrm{m}-\mathrm{Hg}$, respectively. The spectra of $\mathrm{film} \# 20 \mathrm{C}$ is very similar to that of \#19A in that they have the characteristics of highly stoichiometric $\mathrm{Cu}_{x} s$ even though the latter was sputtered in a more sulfur-rich environment. The sulfur content in the $15 \mu \mathrm{m}-\mathrm{Hg}$ mixture appears to be at a critical level for forming stoichiometric $\mathrm{Cu}_{2} \mathrm{~S}$. When the pressure is reduced to $10 \mu \mathrm{m}-\mathrm{Hg}$ excess copper appears as nodular precipitates. An SEM micrograph of a portion of the copper excess film is shown in Fig. 36a and a close-up view of a copper nodule in Fig. 36b. The nodules were not present in sufficient number to affect optical measurements. 

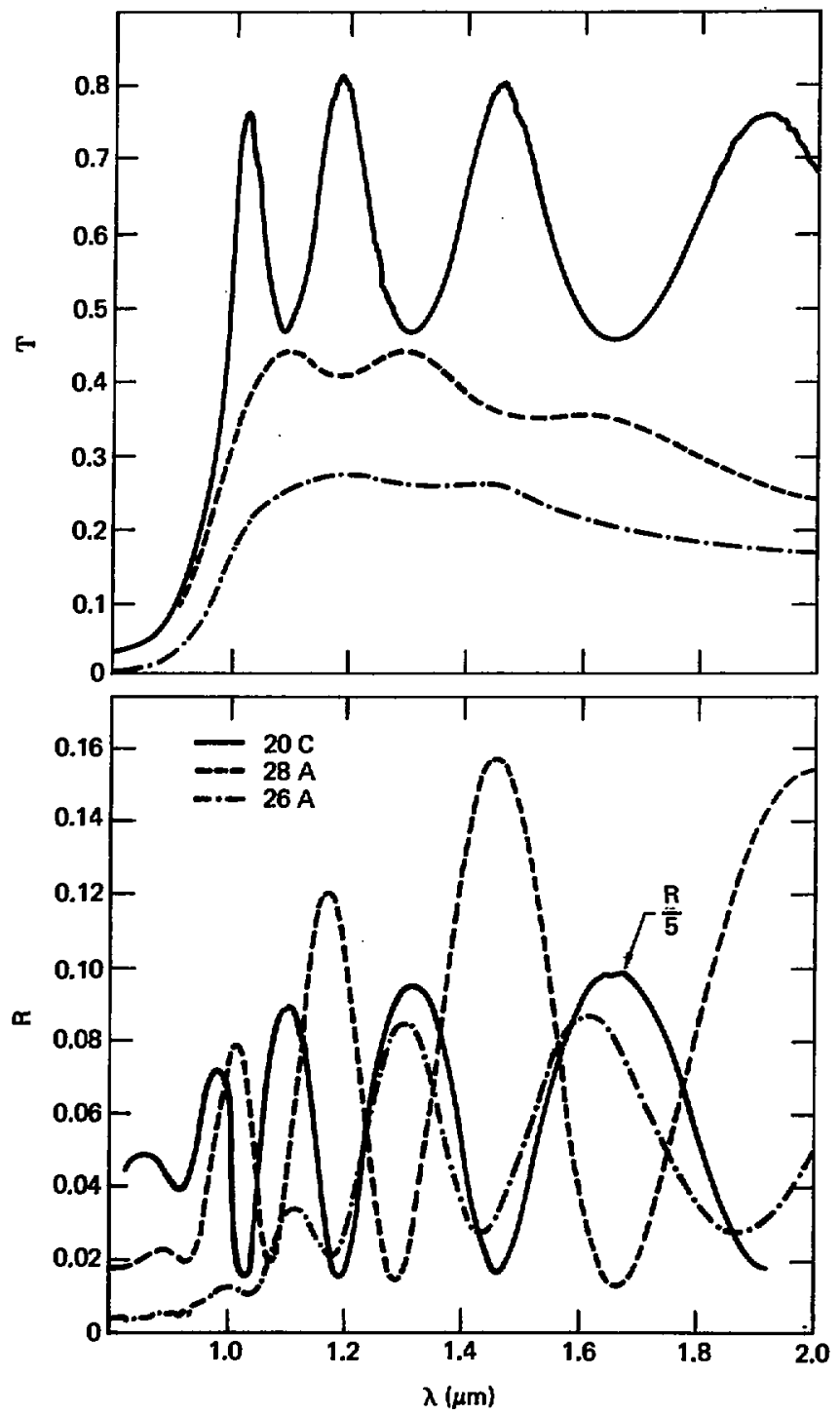

Figure 35. Tranamittance and reflectance of $\mathrm{Cu}_{\mathrm{x}} \mathrm{S}$ sputtered in different preasures. 

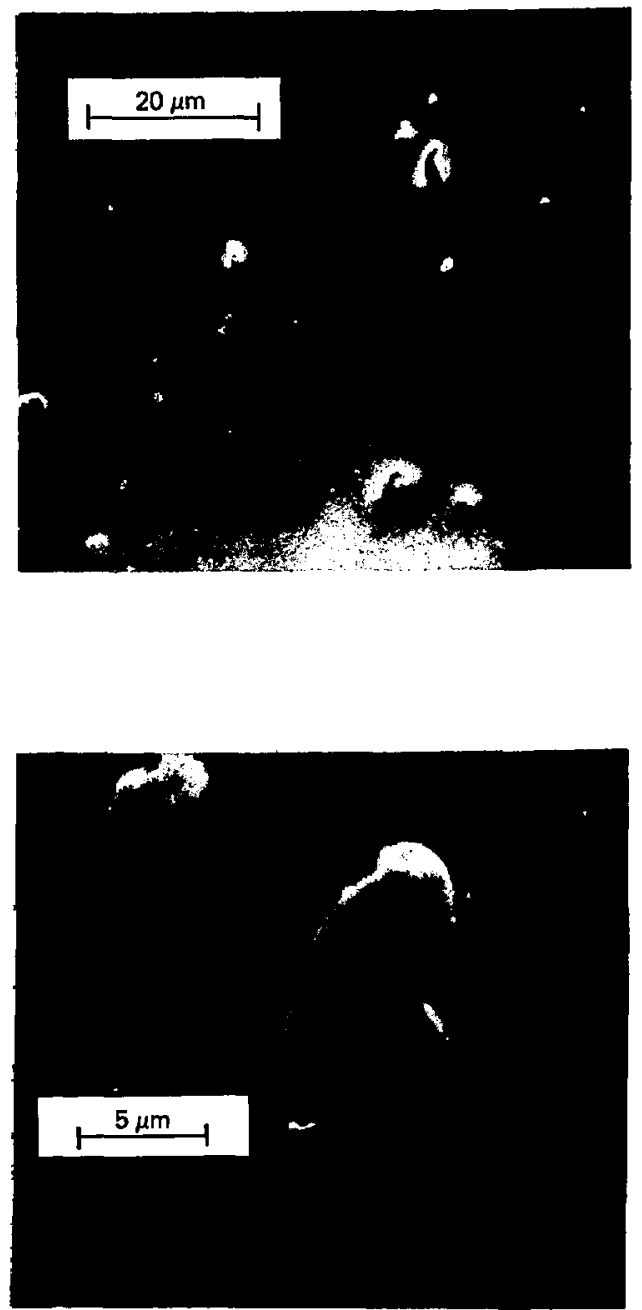

Figure 36. SEM micrographs of copper nodules. 
The spectra of the other two films are significantly different. The lower long wavelength transmittance and suppression of the interference pattern is due to increased free carrier absorption. The concentration of free carriers increased for higher sputter gas pressures. The band edges of these films are less sharp. The higher concentration of impurity states leads to increased band tailing. In fact, the most degenerate film ( $\$ 26 \mathrm{~A})$ appears to have the highest absorbance. More likely it is due to scattering of the incident light. The free surface of the film was noticeably more textured than that of the others.

These conclusions are supported by the absorption spectra, shown in Fig. 37. The $\mathrm{E}^{-3 / 2}$ dependence of the long wavelength absorption indicates that free carrier absorption is assisted by acoustic phonon scattering. This disagrees with the conclusion made in the analysis of the Hall mobility at room temperature. An explanation of this inconsistency will be offered in subsequent discussion of temperature effects on free carrier absorption. After correcting for free carrier absorption the intrinsic absorption spectrum of film \#28A still shows signs of band tailing when compared with that of $\$ 20 \mathrm{C}$.

Plots of $\alpha^{1 / 2}$ and $\alpha^{2}$ versus $h v$ in Fig. 38 shows decreases in the overall absorption and effective bandgaps in going from the highly stoichiometric film $\# 20 \mathrm{C}$ to the more degenerate film $* 28 \mathrm{~A}$. But the small difference in $E_{g}^{\prime}$ is within experimental error between two films sputtered under different conditions. The direct and indirect gaps of the two films are at $1.3 \mathrm{eV}$ and $1.1 \mathrm{eV}$, respectively. The slopes of the $a^{2}$ versus hu curve gives values of $A_{D 1}^{2}=36.7 \times 10^{8} \mathrm{~cm}^{-2}-\mathrm{eV}^{-1}$ and $A_{D 2}^{2}=23.8 \times 10^{8} \mathrm{~cm}^{-2}-\mathrm{eV}^{-1}$ for films *20C and $\# 28 \mathrm{~A}$, respectively. The corresponding electron effective mass, using Eq. (4-1) is $1.1 \mathrm{~m}_{0}$ and $0.82 \mathrm{~m}_{\mathrm{o}}$, respectively. The computed bandgaps and effective masses of these two films are in excellent agreement with that of Film $\# 19 \mathrm{~A}$. 


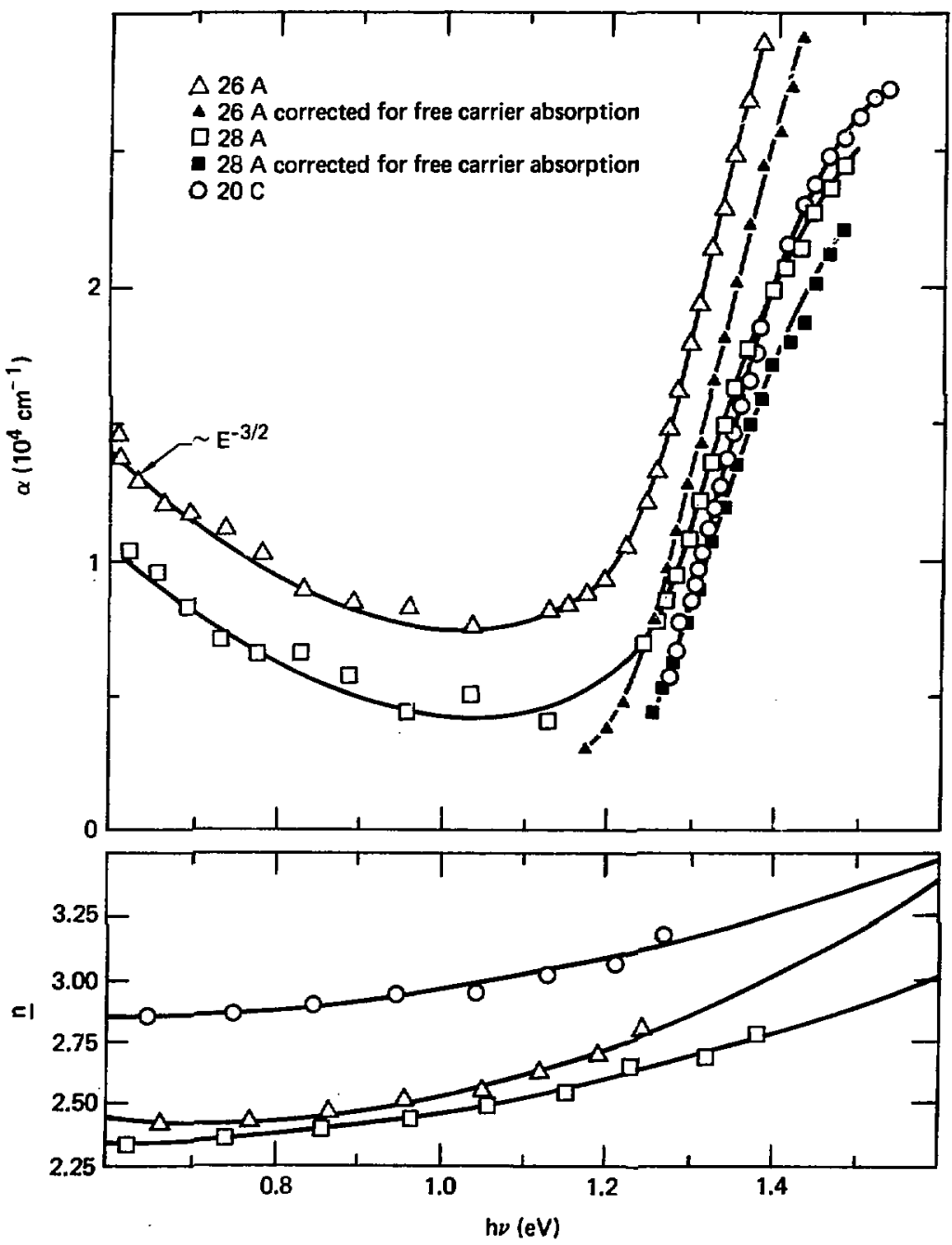

Figure 37. Absorption coefficient and index of refraction of $\mathrm{Cu}_{x} \mathrm{~S}$ films sputtered at different pressures. 


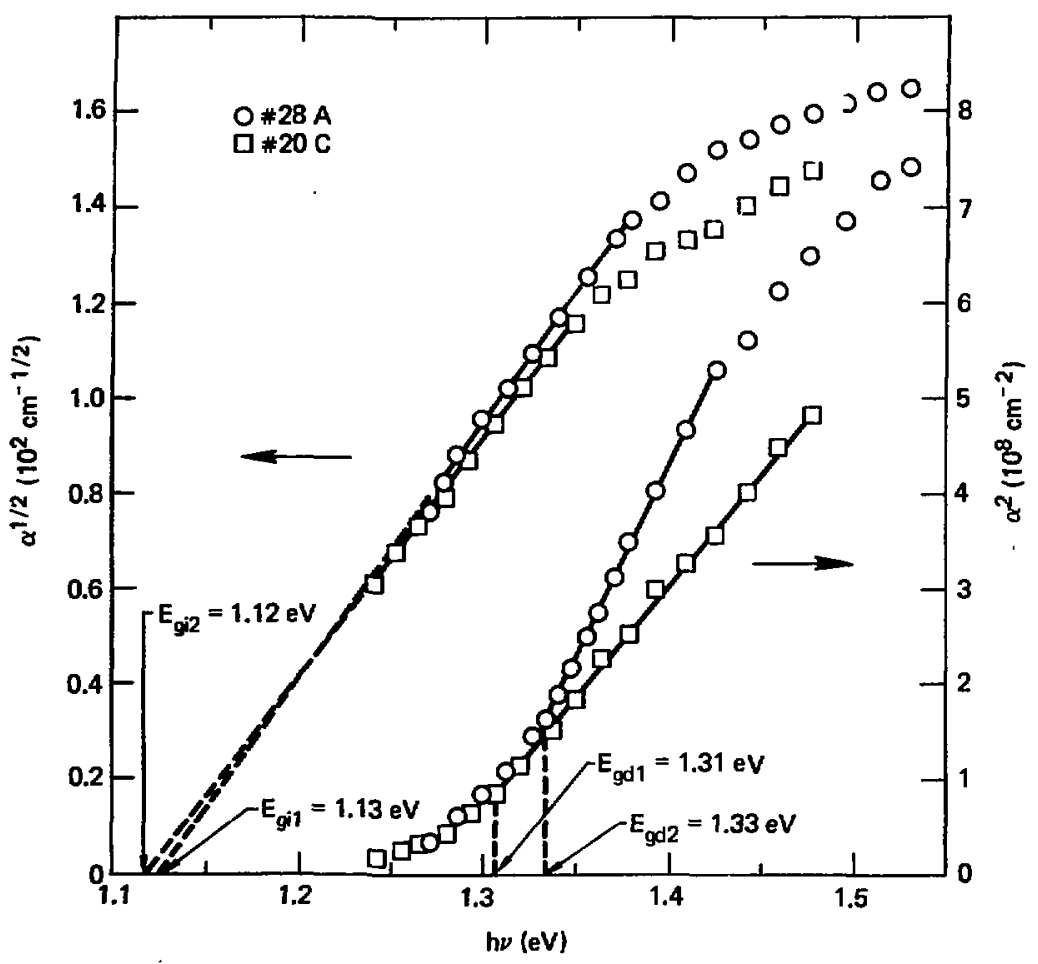

Figure 38. Effects of sputter gas pressure on band gaps of $\mathrm{Cu}_{x} \mathrm{~S}$ films. 
In Fig. 37 film 非 $28 \mathrm{~A}$ has a lower index of refraction than film \#20C. This is consistent with its lower overall reflectance and can be explained by a smaller ratio of $\mathrm{Cu}_{2} \mathrm{~s}$ to djurleite. One would expect film $\# 26$ A to have the lowest index of refraction based on the fact that it has the lowest reflectance and the assumption that it contains the wost sulfur rich mixture of $\mathrm{Cu}_{x} \mathrm{~s}$. Instead, its index of refraction is

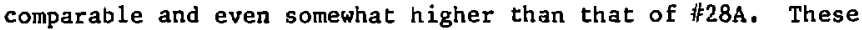
inconsistent results are probably due to errors in estimating the positions of the interference extrema. The high free carrier absorption may have distorted the interference pattern so much that the observed extrema may not correspond to the phase shift simply equal to integer multiples of $\pi$. Also, the textured surface may have resulted in an effective film thickness that is slightly larger than the measured value. All these sources of error can contribute to the small deviation from the expected value.

Hall effect data of films $\# 19 B$, 非6A, and $\# 28 \mathrm{~A}$ are presented in Figs. 39-41. It was impossible to make Hall measurements on film $\# 20 \mathrm{C}$ because of a non-saturating drift in the Hall voltage. This problem was encountered with other films sputtered at low pressures. The drift persisted for at least 45 minutes. The rate of change was greatest during the first two minutes with the voltage changing by $10 \mu \mathrm{V}$ in less than a second, then slowing down to about $2 \mu \mathrm{V}$ in one second. The change was not monotonic. When readings were checked after 15 minute intervals the drift sometimes had reversed directions. This condition was probably due to the presence of an ionic current similar to that reported in early Japanese studies. $[44,47,48,49,50]$ The higher hole concentration and corresponding lower resistivity for films sputtered at higher gas pressures supports earlier statements made with regards to free carrier absorption and deviation from stoichiometry. The best fit to the Hall mobility of film $\$ 28 \mathrm{~A}$ is ionized impurity 


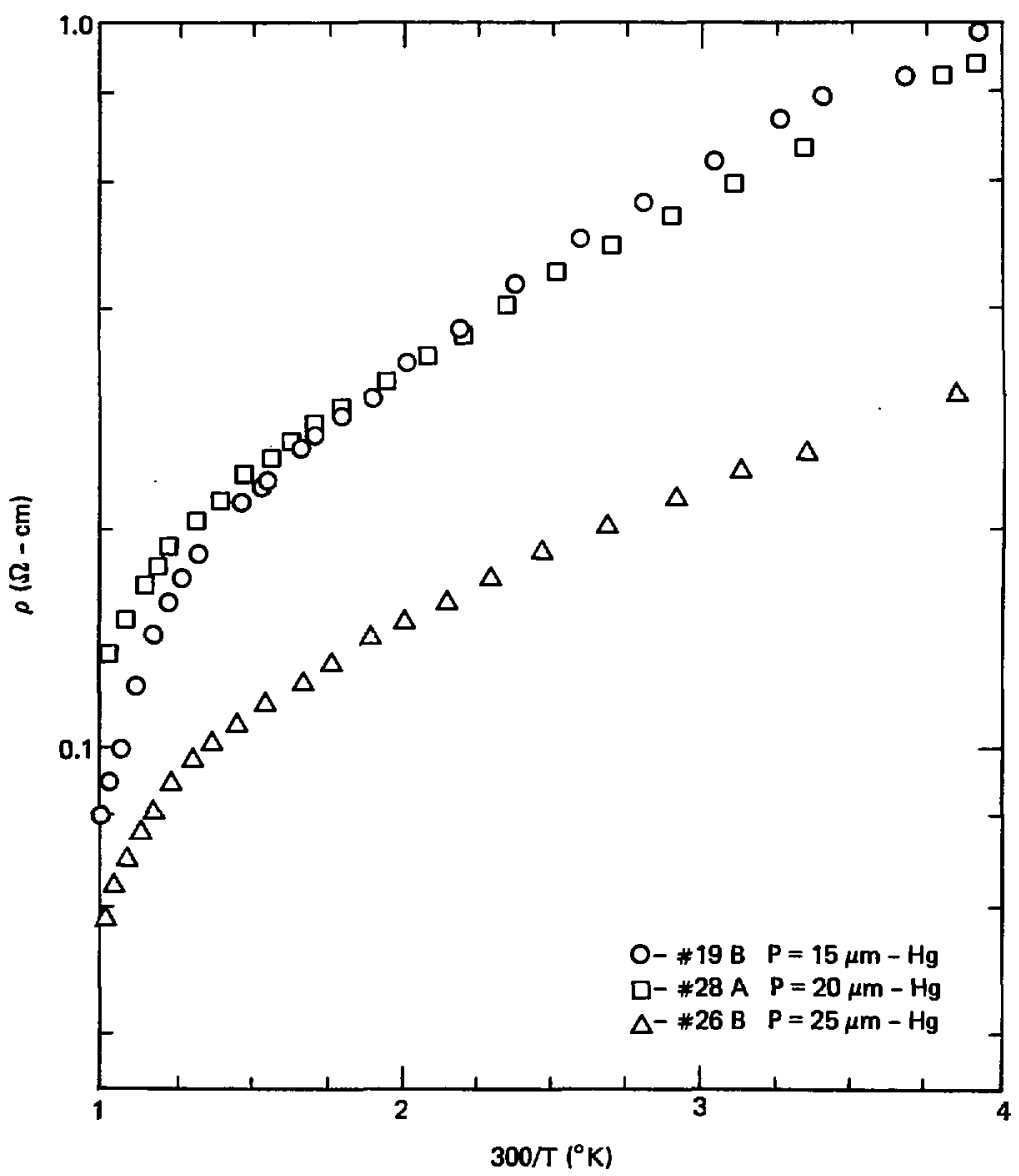

Figure 39. Resistivity of $\mathrm{Cu}_{x} \mathrm{~S}$ films sputtered at different $\mathrm{H}_{2} \mathrm{~S} / \mathrm{Ar}$ pressures. 


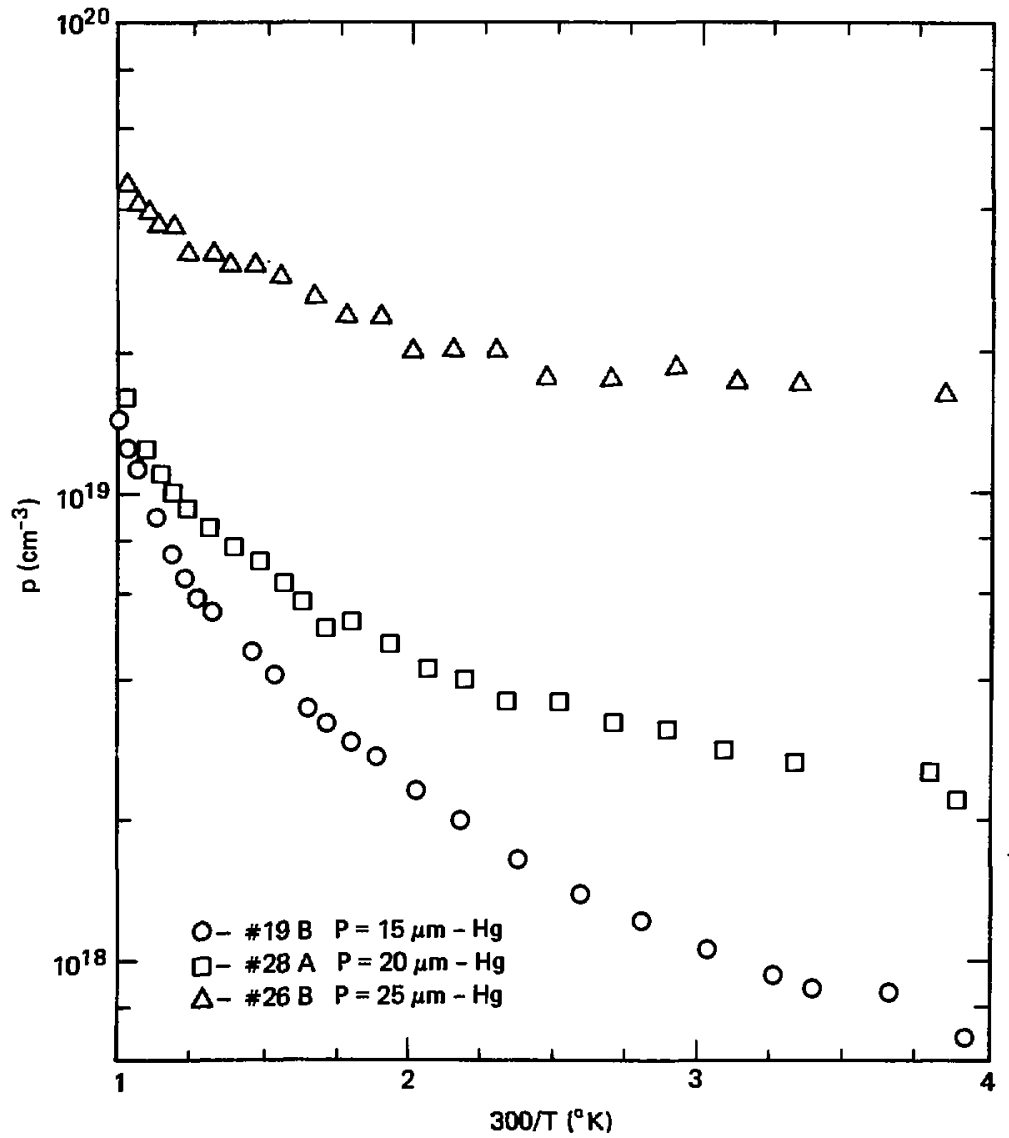

Figure 40. Hole concentration of $\mathrm{Cu}_{x} \mathrm{~S}$ films sputtered at different $\mathrm{H}_{2} \mathrm{~S} / \mathrm{Ar}$ pressures. 


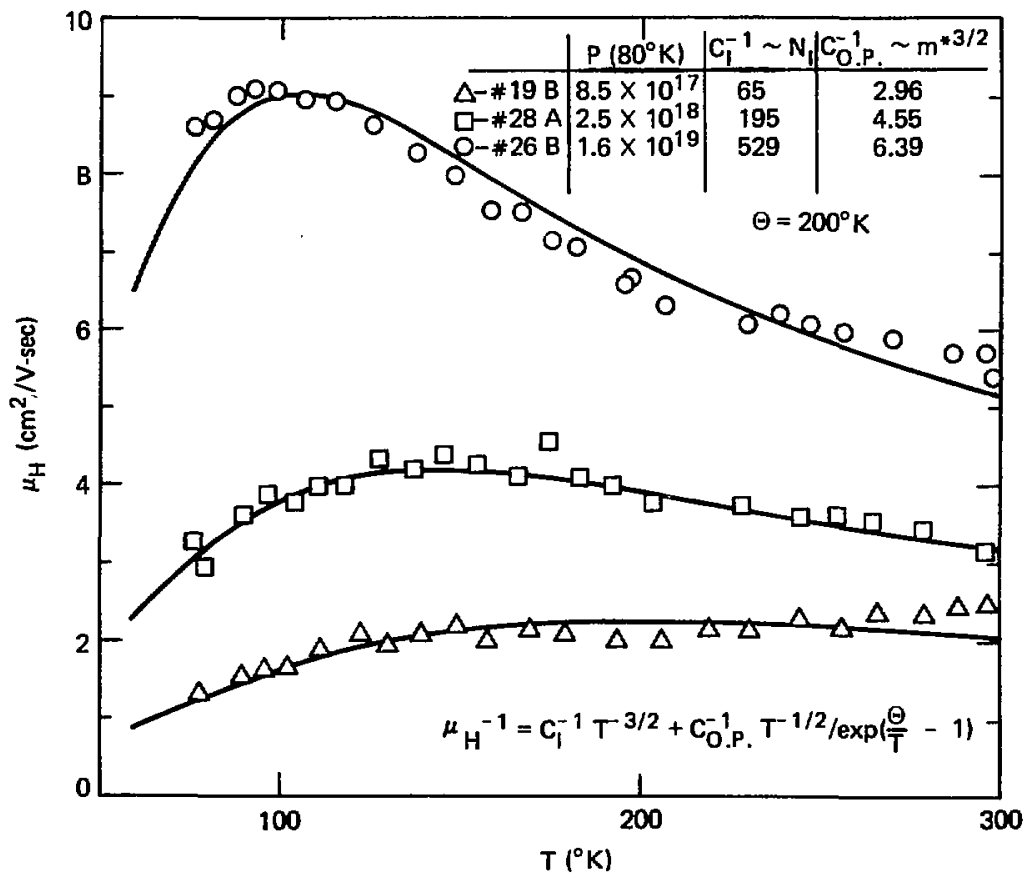

Figure 41. Hall mobility of $\mathrm{Cu}_{x} \mathrm{~S}$ sputtered at different pressures. 
scattering at low temperatures $\left(<100^{\circ} \mathrm{K}\right)$ and polar optical phonon scattering at high temperatures $\left(>150^{\circ} \mathrm{K}\right)$ similar to the results of analyzing film \#19B. Film \#26B can also be fitted to the same two mechanisms even though the characteristic negative slope of phonon scattering is not present. Apparently, the high concentration of ionized impurities have resulted in impurity scattering extending into high temperatures where carriers normally are too energetic to be affected by impurities.

Assuming that the concentration of holes is equal to that of ionized acceptors there should be a direct correlation between the concentration of the latter with the low temperature mobility. According to Eq. (3-32) the mobility due to ionized impurity scattering should be proportional to the ionized acceptor concentration. The three and ten-fold increases in the fitting coefficient as the pressure increased is supported by three and ten-fold increases in free carrier concentration at the same low temperature $\left(\mathrm{T}=100^{\circ} \mathrm{K}\right)$. In the high temperature regime there is an increase in hole effective mass associated with more degenerate films. The increase in the conductively effective mass may be due, in part, to a larger polaron effective mass resulting from the greater polarizability of the more degenerate phase. [128] 


\section{B. Effects of Heat Treatment}

The general consensus with regards to the effect of heat treatments in air and $\mathrm{H}_{2}$ is that the former oxidizes free $\mathrm{Cu}$ extracted from $\mathrm{Cu}_{\mathrm{x}} \mathrm{S}$ while the latter causes the reverse effect by reducing the oxide. $\{15,19,129-132\}$ A1l of these studies were performed on $\mathrm{Cu}_{\mathrm{x}} \mathrm{S}$ films converted from CdS, and with the exception of cook ${ }^{[19]}$ and Fagen, $[132]$ the conclusions were based on measurements made on completed cells. So, the interpretation of the observed effects involves some assumptions about how other cell parameters may have been affected by the heat treatments. In the current study optical absorbance and the Hall effect are measured on isolated $\mathrm{Cu}_{x}$ s sputtered on glass slices. The resulting changes in optical and electrical properties are correlated with changes in chemical phase deduced from $x$-ray diffraction studies.

Figure 42 shows the transmittance and reflectance of film 非8B after heat treatment in $180^{\circ} \mathrm{C}$ air for various time intervals. The effects are an increase in free carrier absorption and broadening of the absorption edge. The heat treatments were applied in increasing time intervals of $2^{\prime}, 5^{\prime}, 15^{\prime}, 98^{\prime}$ for a total of two hours. The effects nearly saturates within the first two minutes, implying that the oxidation may be a self-limiting surface effect. On the other hand, a considerable amount of the small grained film (average grain size on the order of $0.1 \mathrm{~mm}$ ) may be affected because of the high surface to bulk ratio.

The diminished reflectance and shifting of the interference extrema to shorter wavelengths is consistent with the lower indices of refraction of the more degenerate phases. The unexpected increase in reflectance after the final heat treatment indicates the possibility of a slow underlying positive effect of the heat treatments. More comments on this effect will be made in a discussion of longer heat treatments. 

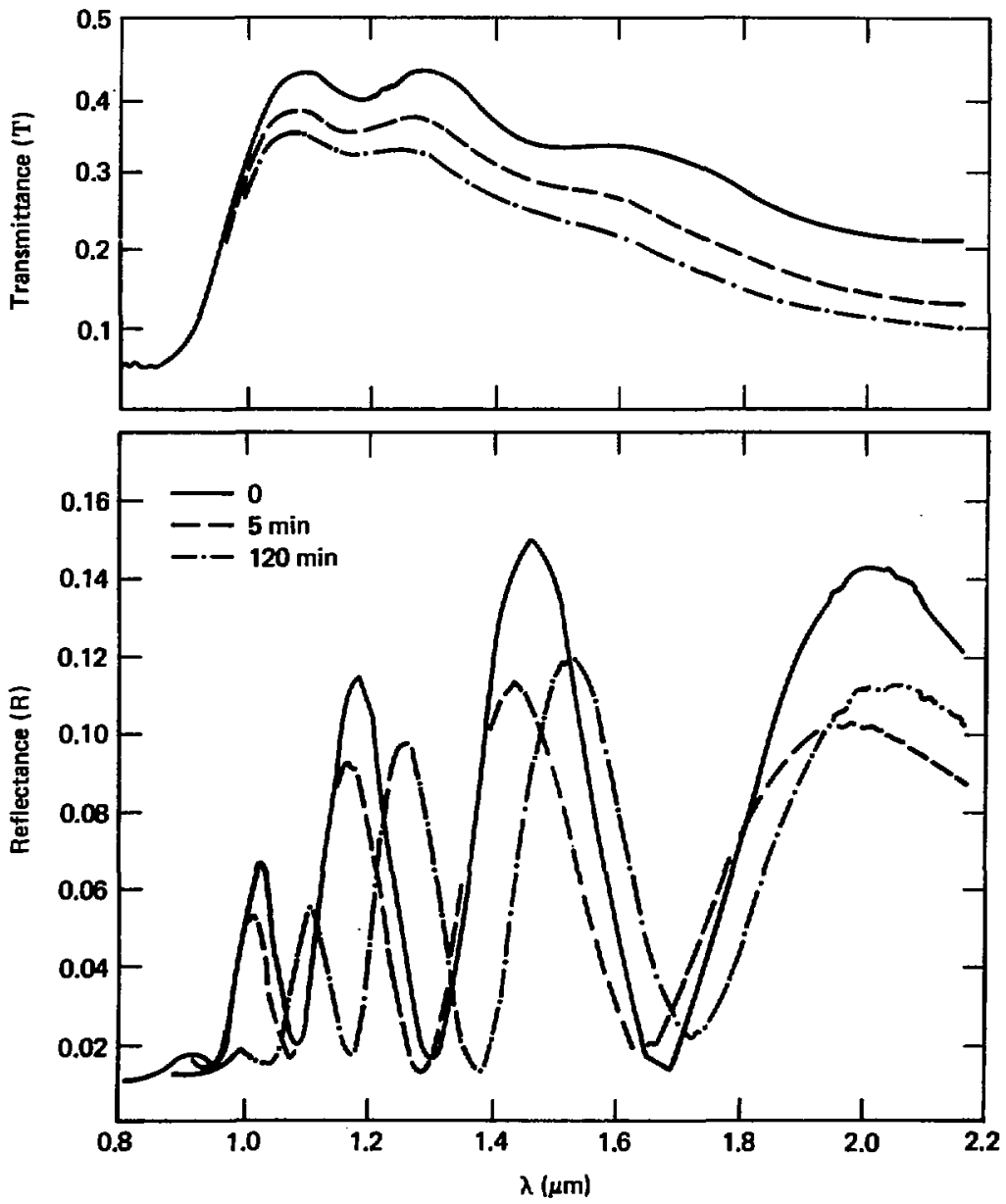

Figure 42. Effects of air heat treatments on $\mathrm{Cu}_{\mathrm{x}} \mathrm{S}$ transmittance and reflectance. 
The transmittance and reflectance of $\mathrm{film} \# 28 \mathrm{C}$ which had undergone heat treatments in $\mathrm{H}_{2}$ /Ar are presented in Fig. 43. The heat treatments were carried out in 30-minute intervals for the same total time as the air heat treated sample. The transmittance and reflectance increased with time and at a slower rate than the degradation process in air. The improvements began to saturate during the final 30 minutes.

The absorption spectra of the two heat treated samples are compared with that of an untreated sample in Fig. 44. All three samples had been deposited in the same sputter run with a total gas pressure of $20 \mu \mathrm{m}-\mathrm{Hg}$, and stored in a plastic case with free flowing $\mathrm{N}_{2}$ when they were not undergoing heat treatments or measurements. All were $1.05 \mathrm{\mu m}$ thick. The air heat treated sample has more free carrier absorption. The $\mathrm{E}^{-2}$ dependence of the long wavelength absorption spectra suggests that polar optical phonons and even ionized impurities may be playing a more important role in the scattering process. After correcting for free carrier absorption the effects of the air and $\mathrm{H}_{2} / \mathrm{Ar}$ heat treatments are to decrease and increase, respectively, the intrinsic absorption. A graphic analysis of the $\alpha^{1 / 2}$ and $\alpha^{2}$ versus $h v$, shown in Fig. 45, indicates that the indirect and direct gaps remain nearly fixed at about $1.1 \mathrm{eV}$ and $1.3 \mathrm{eV}$, respectively. From the slopes of the $\alpha^{2}$ versus hv curves one obtains electron density of states effective masses of $0.90 \mathrm{~m}_{0}, 0.82 \mathrm{~m}_{0}$, and $0.69 \mathrm{~m}_{0}$ for films \#28C, $\mathrm{A}$, and $B$, respectively. These calculations are made with the assumption that $\mathrm{m}_{\mathrm{h}}^{*}=1.7 \mathrm{~m}_{0^{-}}^{[127]}$ If, instead, $\mathrm{m}_{\mathrm{e}}^{*}$ was assumed constant then $\mathrm{m}_{h}^{*}$ would have to decrease with increasing degeneracy. In other words, the density of states is decreasing with increasing degeneracy. These results are not entirely consistent with the rigid band model proposed by Mulder ${ }^{[29]}$ in which the only change that is allowed is a varying effective bandgap resulting from the Fermi level being at different positions inside the valence band. Instead, the reverse appears to be true. The bandgap remains relatively constant while the curvature $\left(\frac{\partial^{2} E}{\partial k^{2}} \sim m^{k-1}\right)$ increases with increasing degeneracy. If there is a 

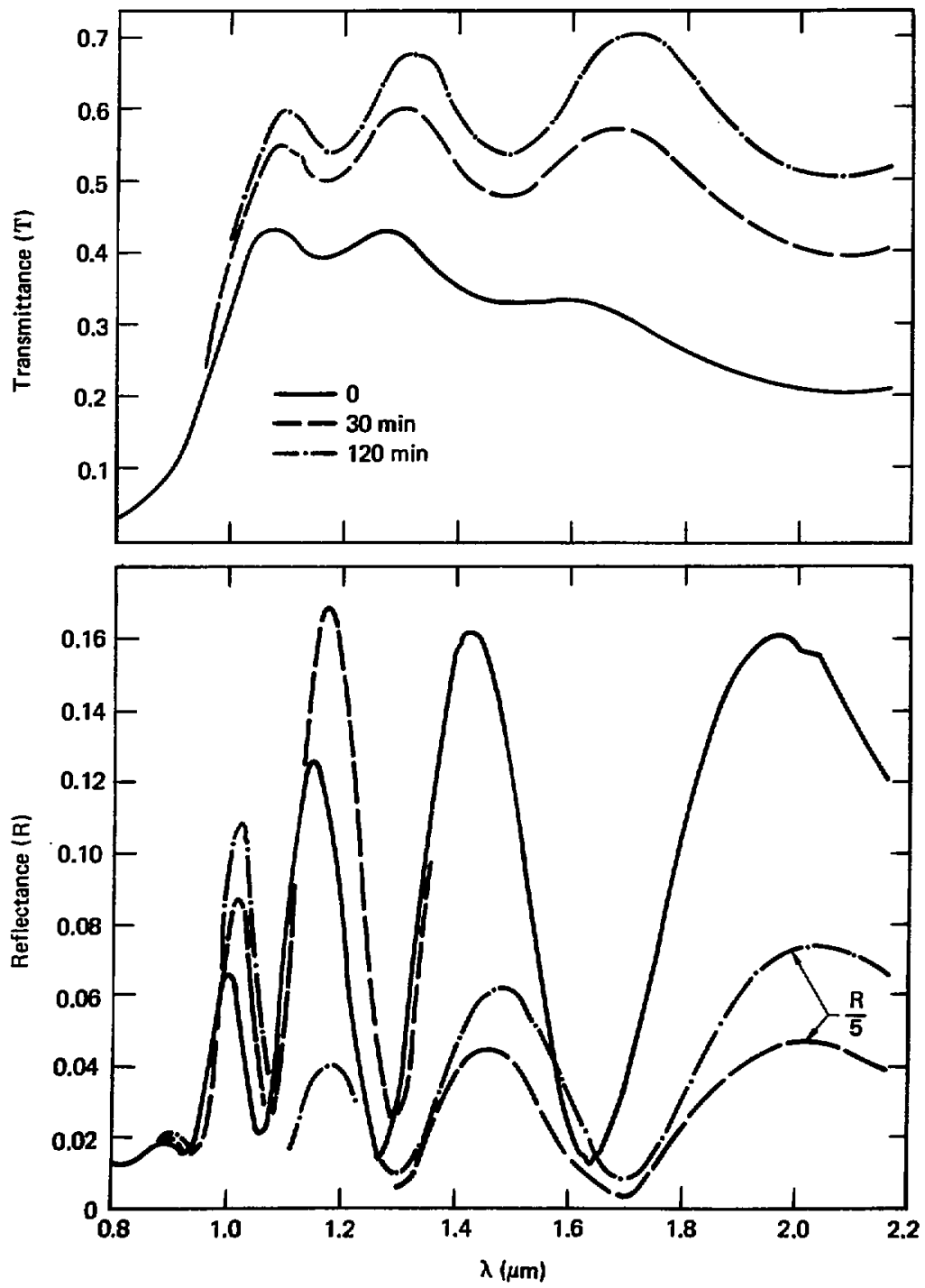

Figure 43. Effects of $\mathrm{H}_{2} / \mathrm{Ar}$ heat treatments on $\mathrm{Cu}_{x} \mathrm{~S}$ transmittance and reflectance. 

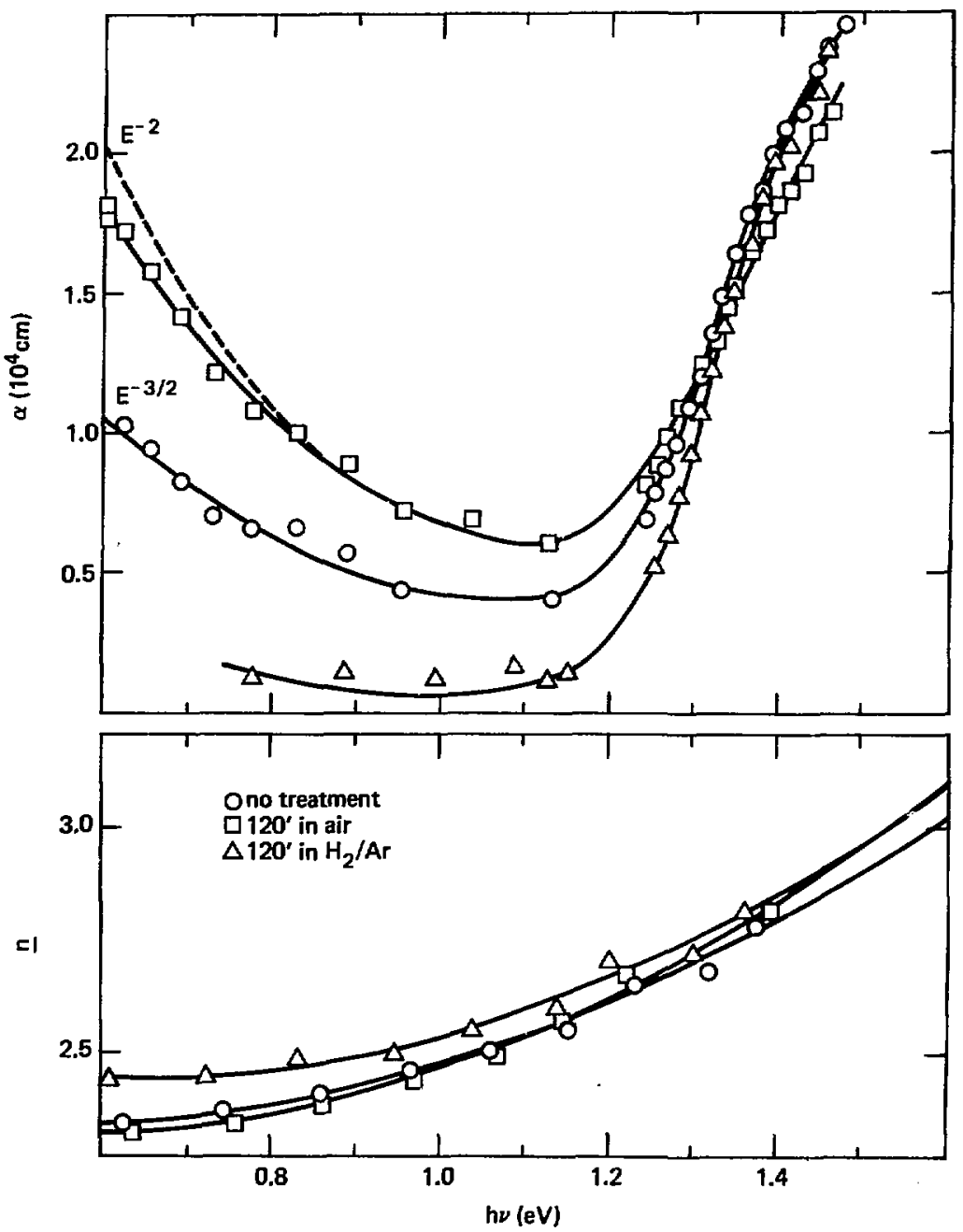

Figure 44. Heat treatment effects on the absorption spectra and indices of refraction in Cu $\mathrm{s}$ films. 


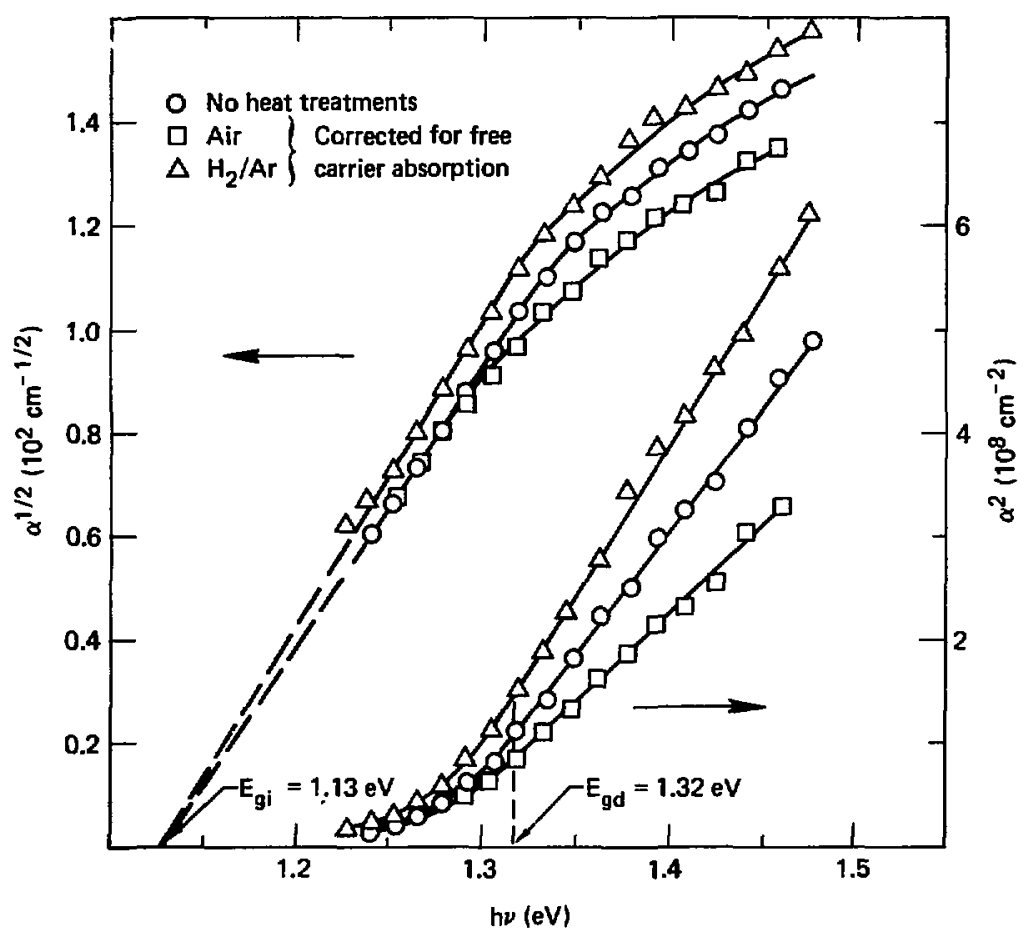

Figure 45. Effects of heat treatments on bandedge. 
Burstein-Moss shift, it is undetectable from the analys is of this data. With $\mathrm{m}_{\mathrm{e}}^{*}=\mathrm{m}_{\mathrm{o}}$ and $\mathrm{p} \simeq 10^{19} \mathrm{~cm}^{-3}, \mathrm{Eq} \cdot 3-4$ gives $\Delta \mathrm{E}=0.01 \mathrm{eV}$ which can easily be obscured by experimental error. A non-rigid band structure appears to be a more zeasonable model. From the differences in the dielectric constants $\left(E \simeq \underline{n}^{2}\right)$ alone, Fig. 5 , one would expect to find significant differences in the crystal fields of the various $\mathrm{Cu}_{\mathbf{x}} \mathrm{s}$ phases.

The results of the Hall measurements offered supporting evidence of the noted changes in the optical data. Figs. 46 and 47 show that the hole concentration and conductivity, respectively, increased with air heat treatment while the reverse occurred with $\mathrm{H}_{2}$ /Ar heat treatment. The Hall mobilities of the holes in the three films are shown in Fig. 48. The best fit of the data is, as in previous discussions, ionized impurity scattering at low temperatures and polar optical phonon scattering at high temperatures. Most of the effects of the heat rreatments occur in the low temperature regime. Ionized impurity scattering increased with air heat treatment and decreased with $\mathrm{H}_{2} / \mathrm{Ar}$ treatment. The differences in ionized impurity concentrations, inferred from the fitting parameter $C_{I}$, agrees with the differences in hole concentration in the three films. At high temperatures the differences in mobility appears to be due to differences in polaron effective masses, since the real effective mass, according to the optical data, actually decreases with increasing carrier concentration.

Partial x-ray diffraction data $[133]$ is presented in Figs. 49 and 50. Fig. 49 is a comparison of a diagnostic djurleite peak and Fig. 50 is that of a diagnostic $\mathrm{Cu}_{2} \mathrm{~S}$ peak of the three films. The data was taken with a Picker $x$-ray diffractometer using a Cu-a source. The peak at $2 \theta=26.5^{\circ}$ corresponds to diffraction by the (804) or (434) planes of djurleite with interplanar distance or lattice parameter (d) of $3.39 \mathrm{~A}$. The peak at $2 \theta=40.9^{\circ}$ is due to the (106) or (522) planes of $\mathrm{Cu}_{2} \mathrm{~S}$ with $\mathrm{d}=2.21 \mathrm{~A}$. The result of two hours in $180^{\circ} \mathrm{C}$ air, after subtracting out the background is a $30 \%$ increase in djurleite 


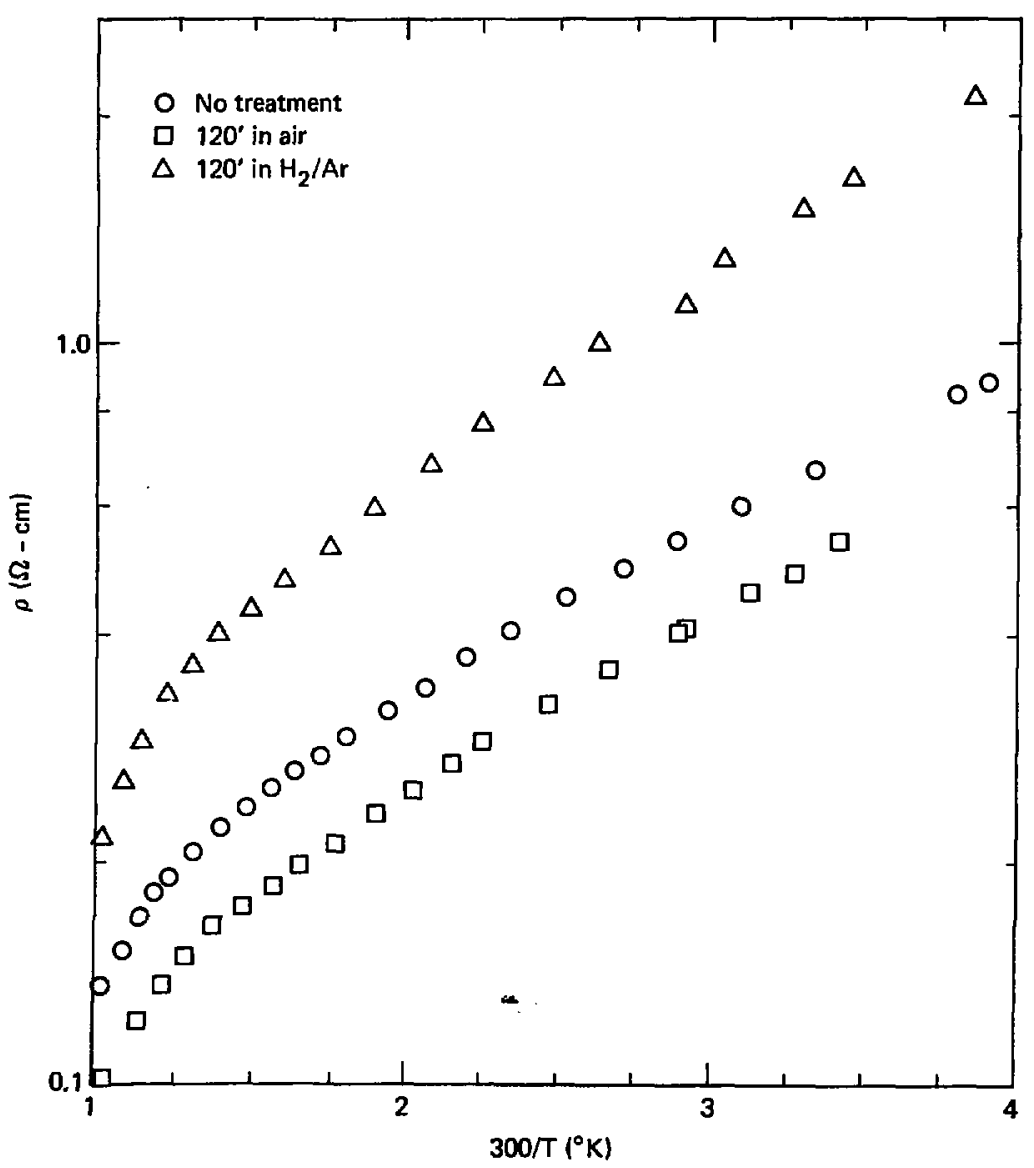

Figure 46. Resistivity of heat treated $\mathrm{Cu}_{\mathrm{x}} \mathrm{S}$ films. 


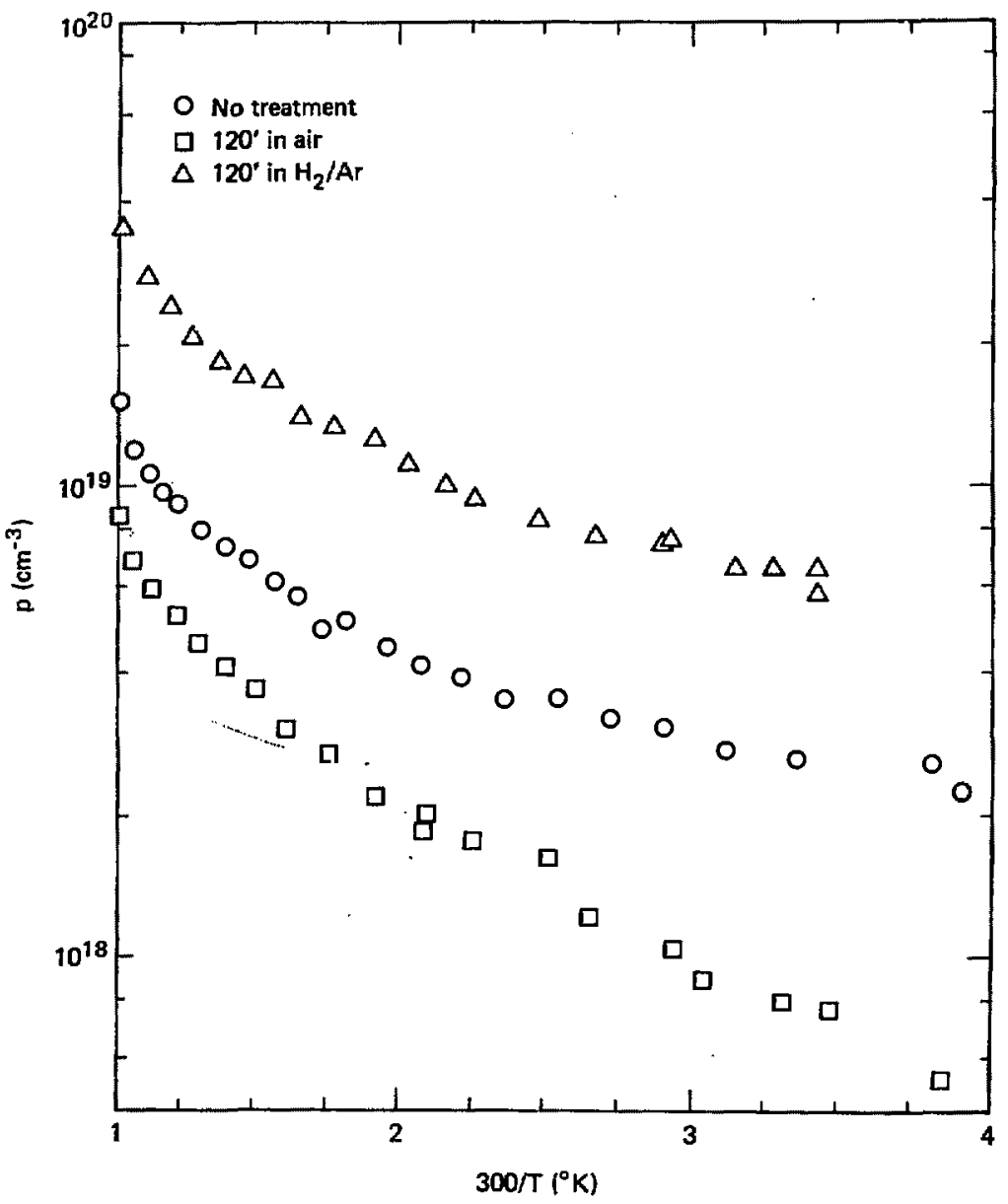

Figure 47. Hole concentration of heat treated $\mathrm{Cu}_{x} \mathrm{~S}$ films. 


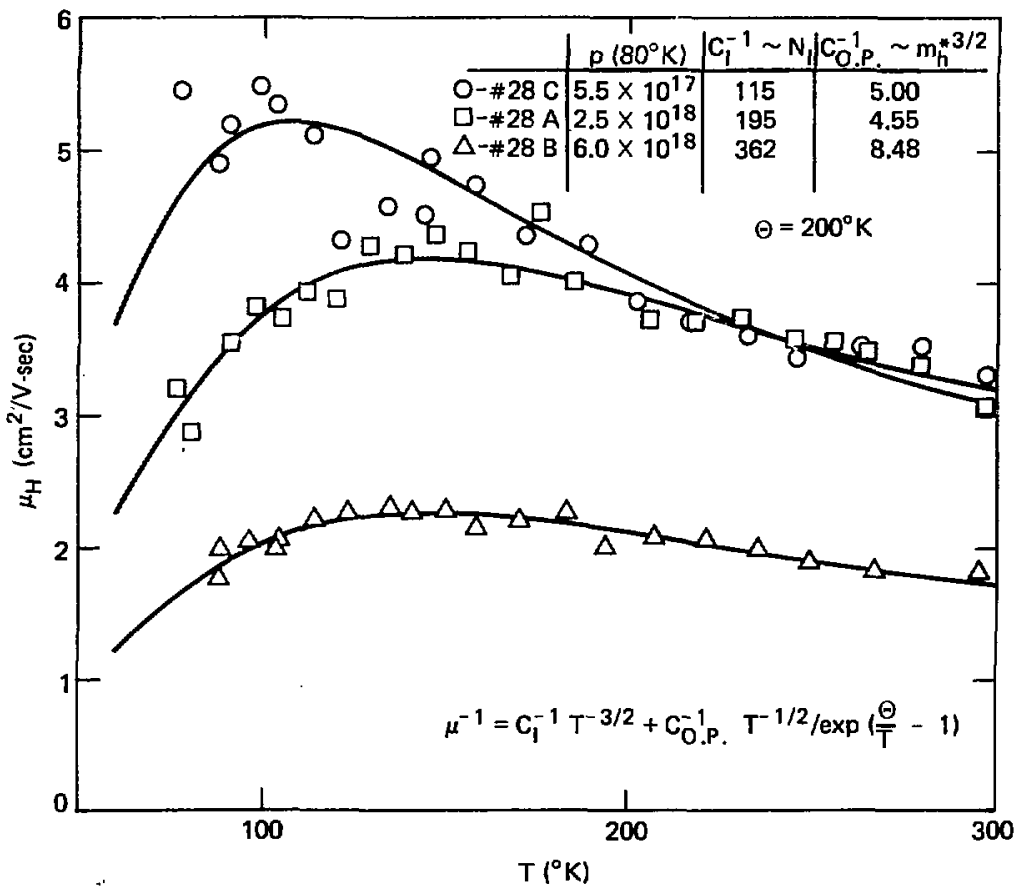

Figure 48. Hall mobility of heat treated $\mathrm{Cu}_{x} \mathrm{~S}$ films. 


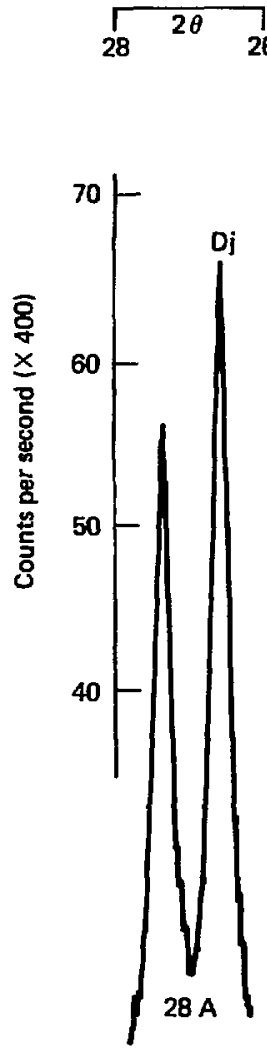

no treatment

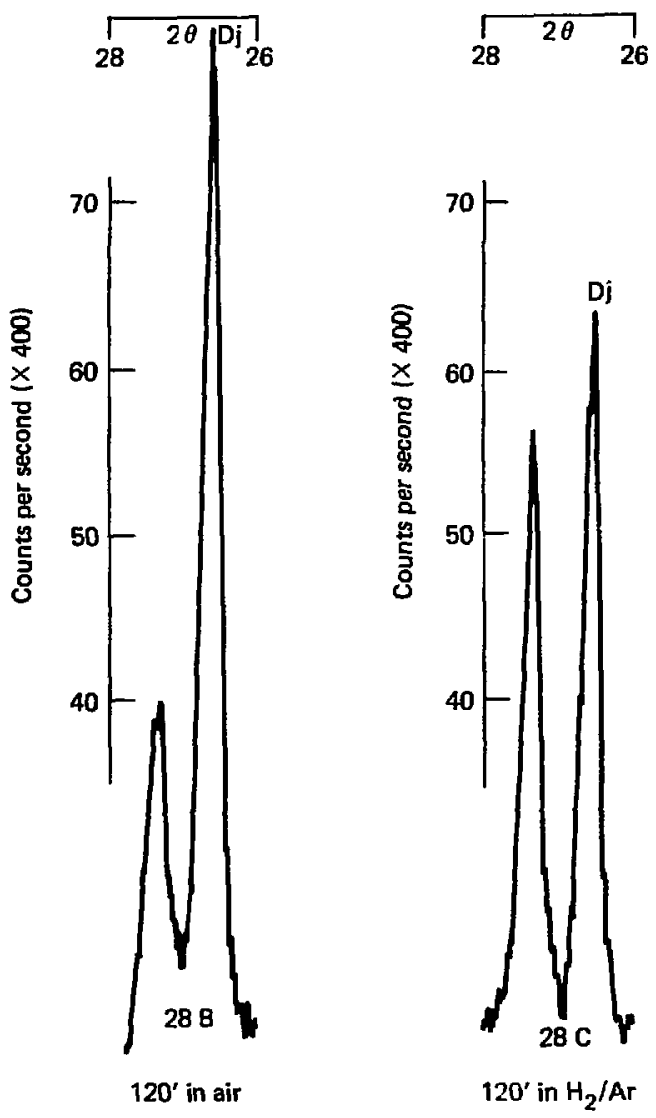

Figure 49. Diagnostic $x$-ray diffraction peaks for djurleite in heat treated films. 

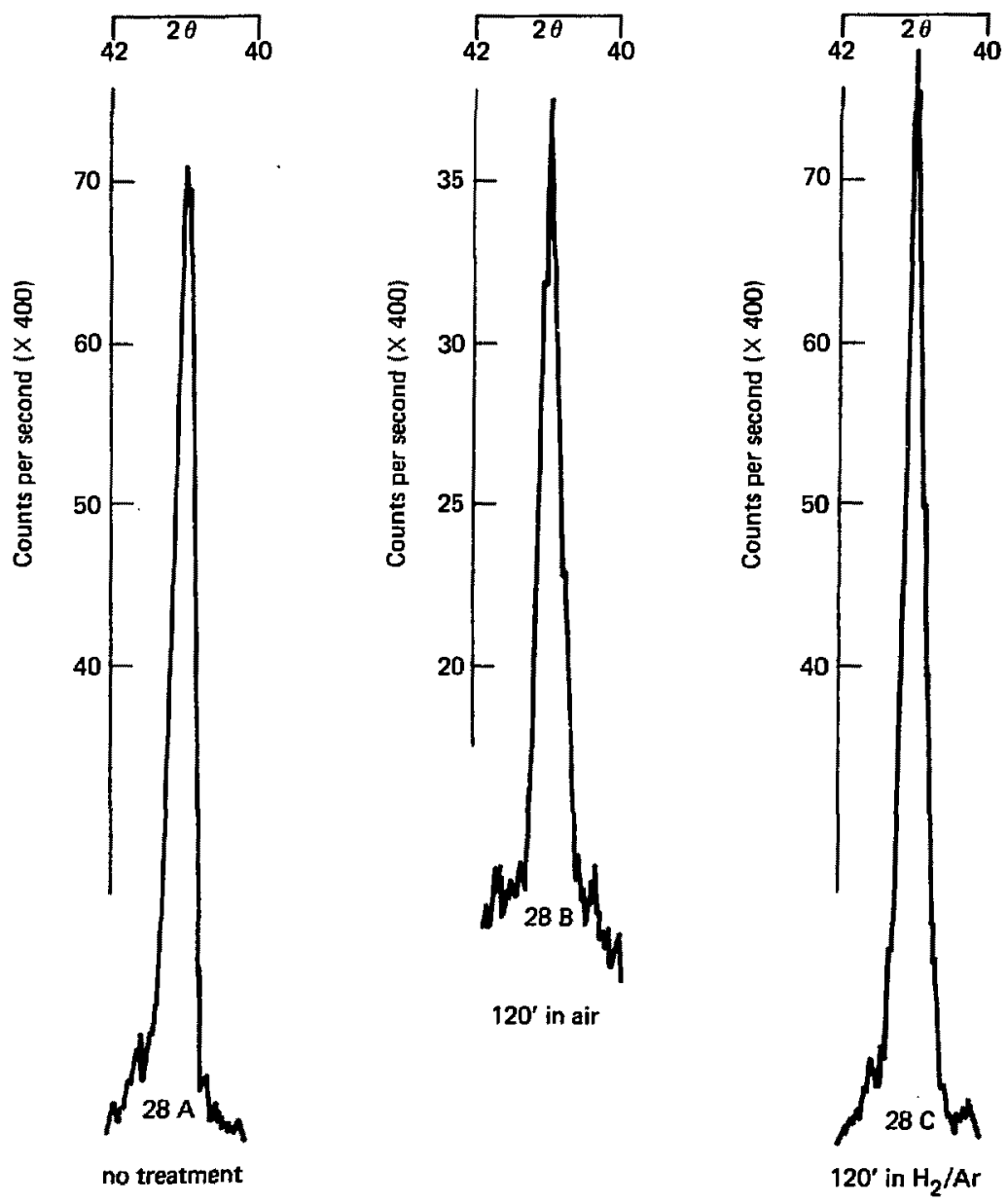

Figure 50. Diagnostic x-ray diffraction peak for $\mathrm{Cu}_{2} \mathrm{~S}$ in heat treated films. 
accompanied by a $56 \%$ loss of $\mathrm{Cu}_{2} \mathrm{~S}$. The reverse occurred, though not to the same extent, with $\mathrm{H}_{2} /$ Ar neat treatment. There was a $7 \%$ loss of djurleite and $10 \%$ increase in $\mathrm{Cu}_{2} \mathrm{~S}$.

Six such studies were undertaken. With the exception of one highly degenerate $\mathrm{film}$, the air heat treatment resulted in degraded optical properties accompanied by an increase in the djurleite peak and a decrease in the $\mathrm{Cu}_{2.0} \mathrm{~S}$ peak. The one exception was a film that was sputtered in $25 \mu \mathrm{m}$ of $\mathrm{H}_{2} \mathrm{~S} / \mathrm{Ar}$. Its optical properties improved during the first 30 minutes, then it started to degrade just like the rest of the films. No $x$-ray data was available for this film. The results of the $H_{2}$ /Ar heat treatments were much less conclusive. The small amount of expected phase changes observed in 非 $28 \mathrm{C}$ corresponds to the largest increase in long wavelength transmittance. But increases in transmittance and the expected phase changes were not always observed. The inconclusive results may be due to a combination of:

1) the inefficiency of molecular $\mathrm{H}_{2}$ as a reducing agent at the low heat treatment temperatures, and 2) the absorption of atmospheric oxygen by the small grained films which would result in oxidization when heat was introduced regardless of the heat treatment ambient. The last hypothesis is supported by early observations that when films were inserted into the hot zone of the $\mathrm{H}_{2} / \mathrm{Ar}$ furnace without thoroughly flushing out the furnace tube with $\mathrm{H}_{2} / \mathrm{Ar}$ their optical properties often degraded.

In order to clarify the scattered results, a set of films were heat treated for a much longer time period. Three $1.2 \mu \mathrm{m}$ films (非 $32 \mathrm{~A}, \mathrm{~B}, \mathrm{C}$ ) were sputtered in $20 \mu \mathrm{m}-\mathrm{Hg}$ of $\mathrm{H}_{2} \mathrm{~S} / \mathrm{Ar}$. The transmittance and reflectance spectra of $\mathrm{film} \$ 32 \mathrm{~A}$, after various time intervals in $180^{\circ} \mathrm{C}$ air, are presented in Fig. 51. Just as in the previous series of experiments the films quickly degraded to some limiting condition in five minutes. Very little change was observed after the first hour but small improvements appeared with continued heating. After eight hours, in 1,2 , and 4-hour intervals, the improvements reach a saturation 1 imit 

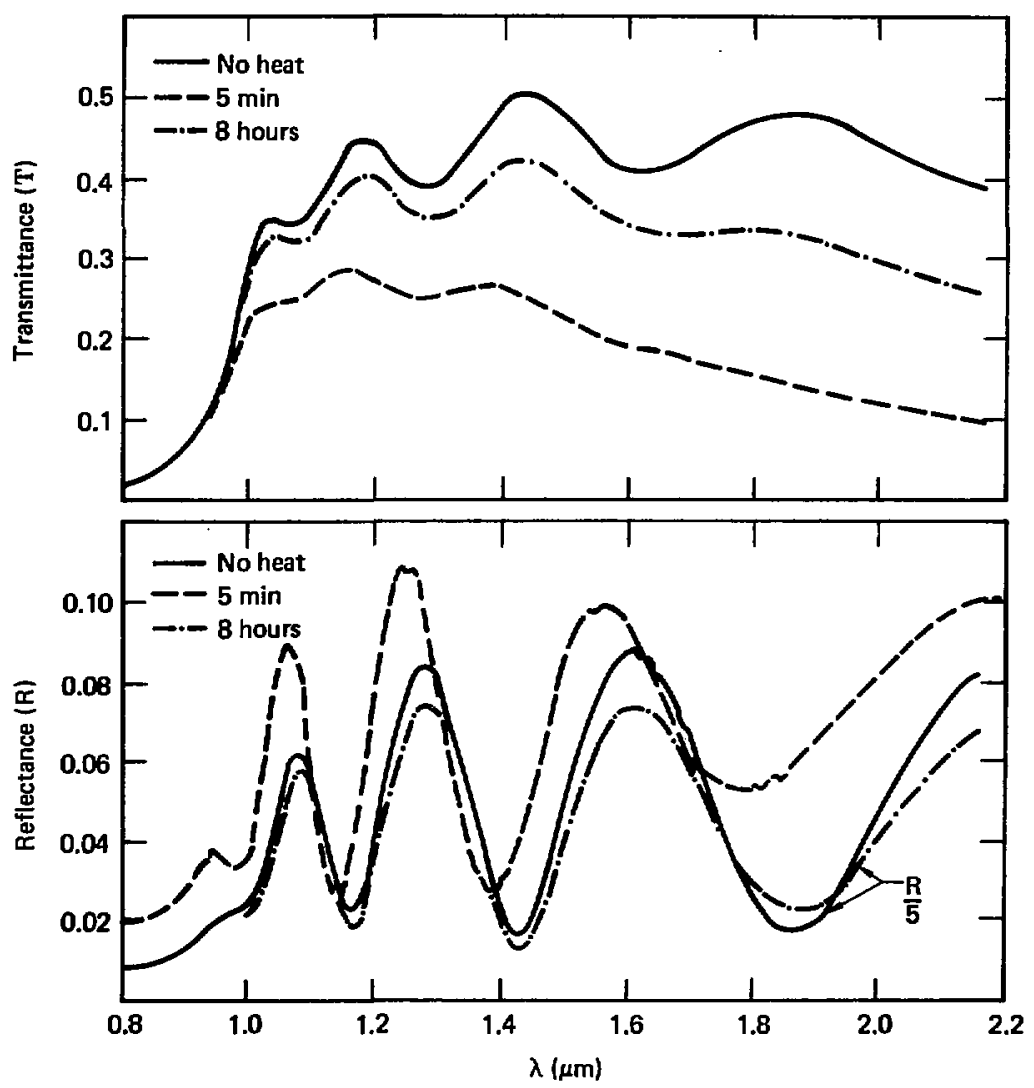

Figure 51. Effects of extended air heat treatment on $\mathrm{Cu}_{x} \mathrm{~S}$ transmittance and reflectance. 
with the film's optical properties restored almost to its initial state. The $x$-ray data of $\$ 32 A$ and an untreated film $\$ 32 B$ is partially reproduced in Fig. 52. There appears to be a $29 \%$ loss of $\mathrm{Cu}_{2} \mathrm{~S}$ with a 24\% increase in djurleite. This data was taken with an $\mathrm{Fe}-\alpha$ source. The peaks are located at different $2 \theta$ values but correspond to the same Bragg planes as those noted in the previous discussion.

Sample \#32C was subjected to a total of 12 hours of heat treatme $\mathrm{I}_{2}$. in $\mathrm{H}_{2}$ /Ar in two initial intervals of $45^{\prime}$ each followed by a single 10.5-hour interval. The transmittance and reflectance spectra are shown in Fig. 53. There is a fairly linear degradation with time for the first $90^{\prime}$. The extent of the degradation is almost the same as the air heat treatment but the rate is considerably lower. The $x$-ray data in Fig. 54 shows a loss of $21 \% \mathrm{Cu}_{2} \mathrm{~s}$ with a $5 \%$ increase in djurleite. After the final 10.5 hours the optical data indicates regeneration beyond the initial state. The net change indicated by a comparison of Figs. $52 \mathrm{a}$ and $54 \mathrm{~b}$ is a decrease in both $\mathrm{Cu}_{2.0} \mathrm{~s}$ and djurleite, the former by $14 \%$ and the latter by $20 \%$. Apparently, the improvements in the optical properties should be correlated to the relative rather than the absolute changes in the $\mathrm{Cu}_{2} \mathrm{~S}$ and djurleite peaks.

The results cannot be fully explained by a simple oxidation and reduction process. A complete theory must take into consideration the evidence that oxidation and reduction appears to be taking place in both types of heat ereatment environments and also account for the differences in the rate of change as well as the final effects. Beneficial effects in air heat treatments had been observed by Fagen. [132] He attributed the improvements to annealing. The removal of physical strains and defects would reduce the density of localized electronic states. This is a plausible explanation, especially for sputtered films. But the observed changes in indices of refraction and lattice constants suggest that the cause may be of a more chemical nature. 


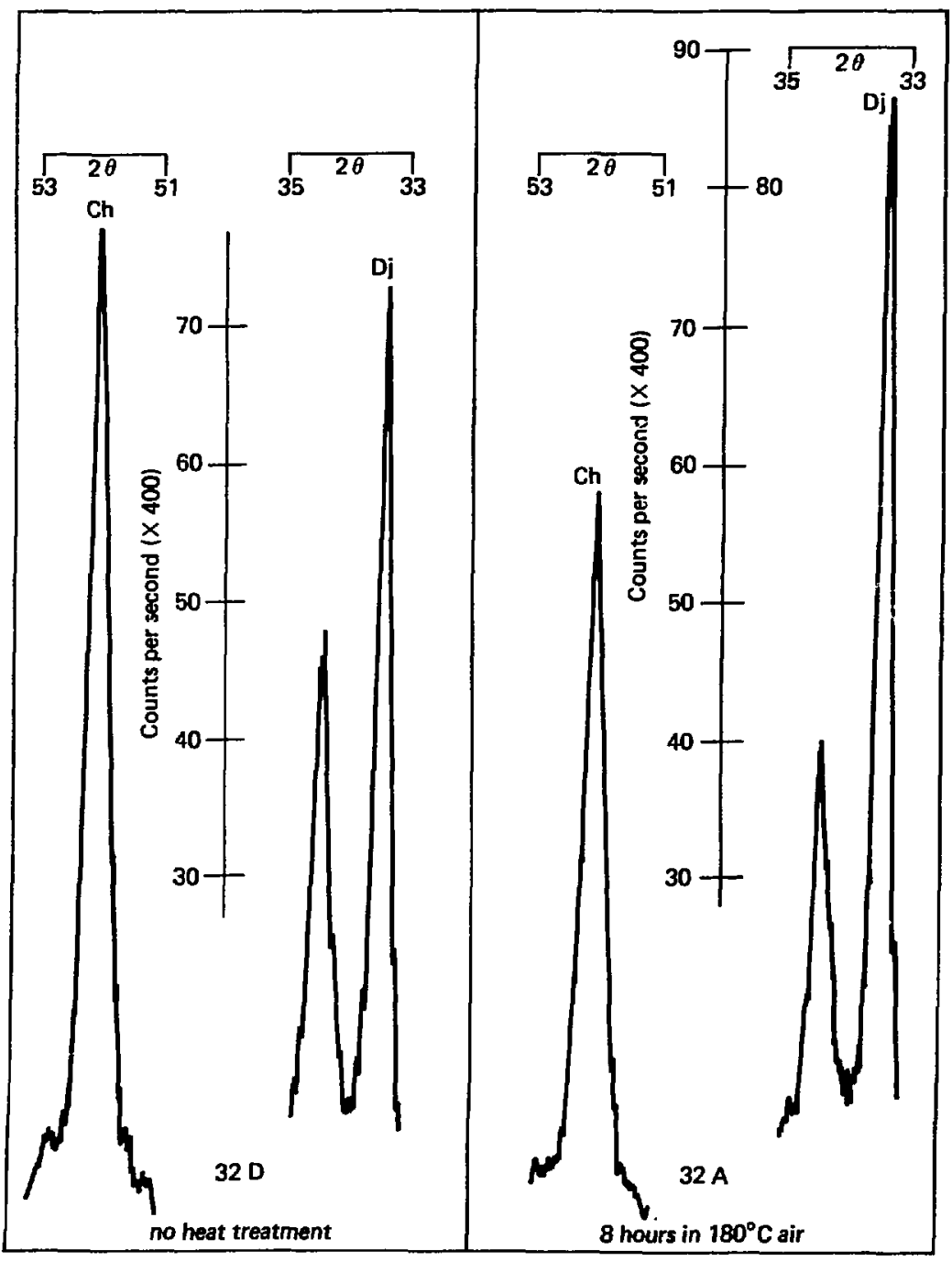

Figure 52. Phase changes due to extended air heat treatment. 

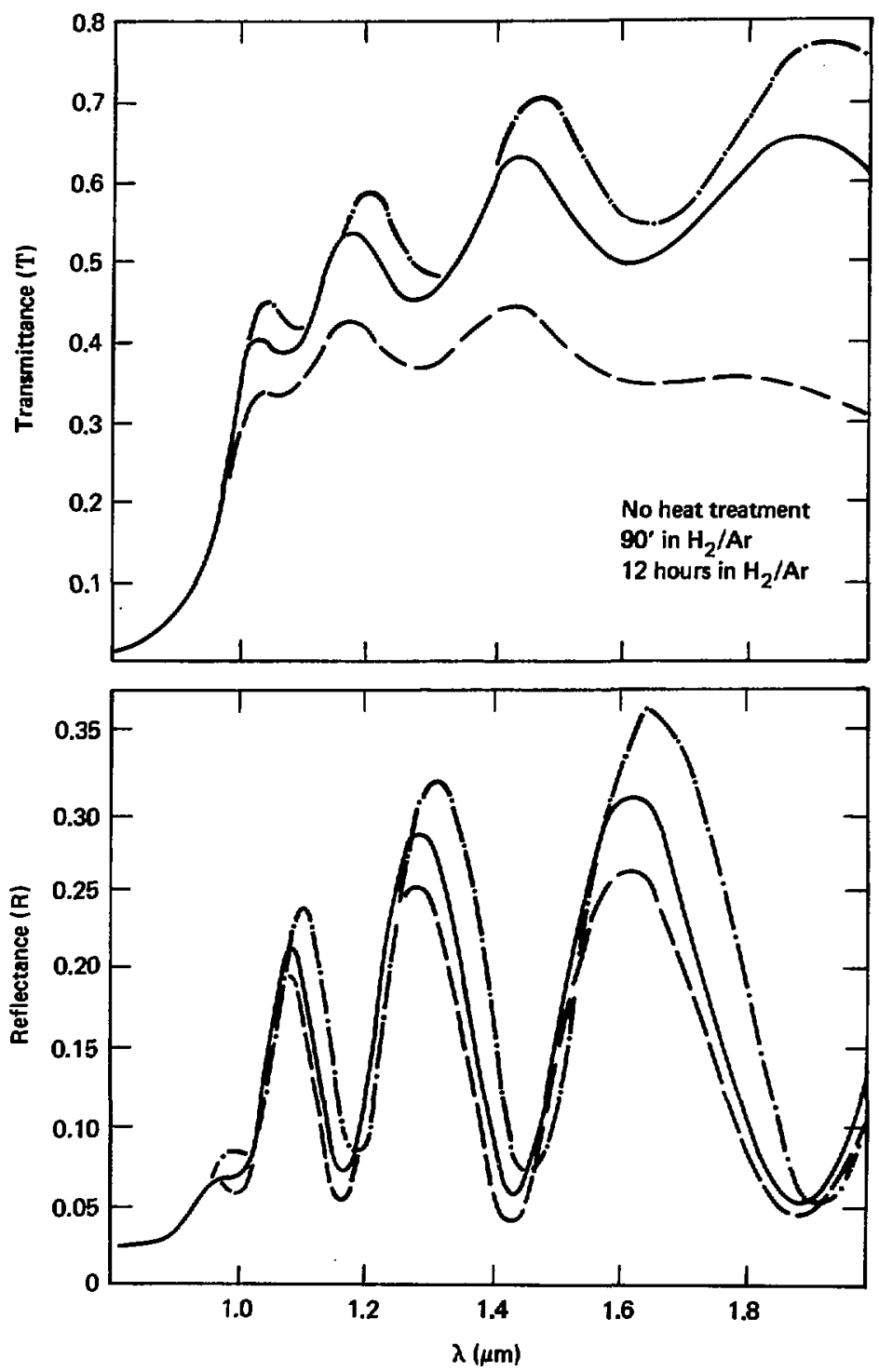

Figure 53. Effects of extended $\mathrm{H}_{2}$ /Ar heat treatment on $\mathrm{Cu}_{x} \mathrm{~S}$ transmittance and reflectance. 


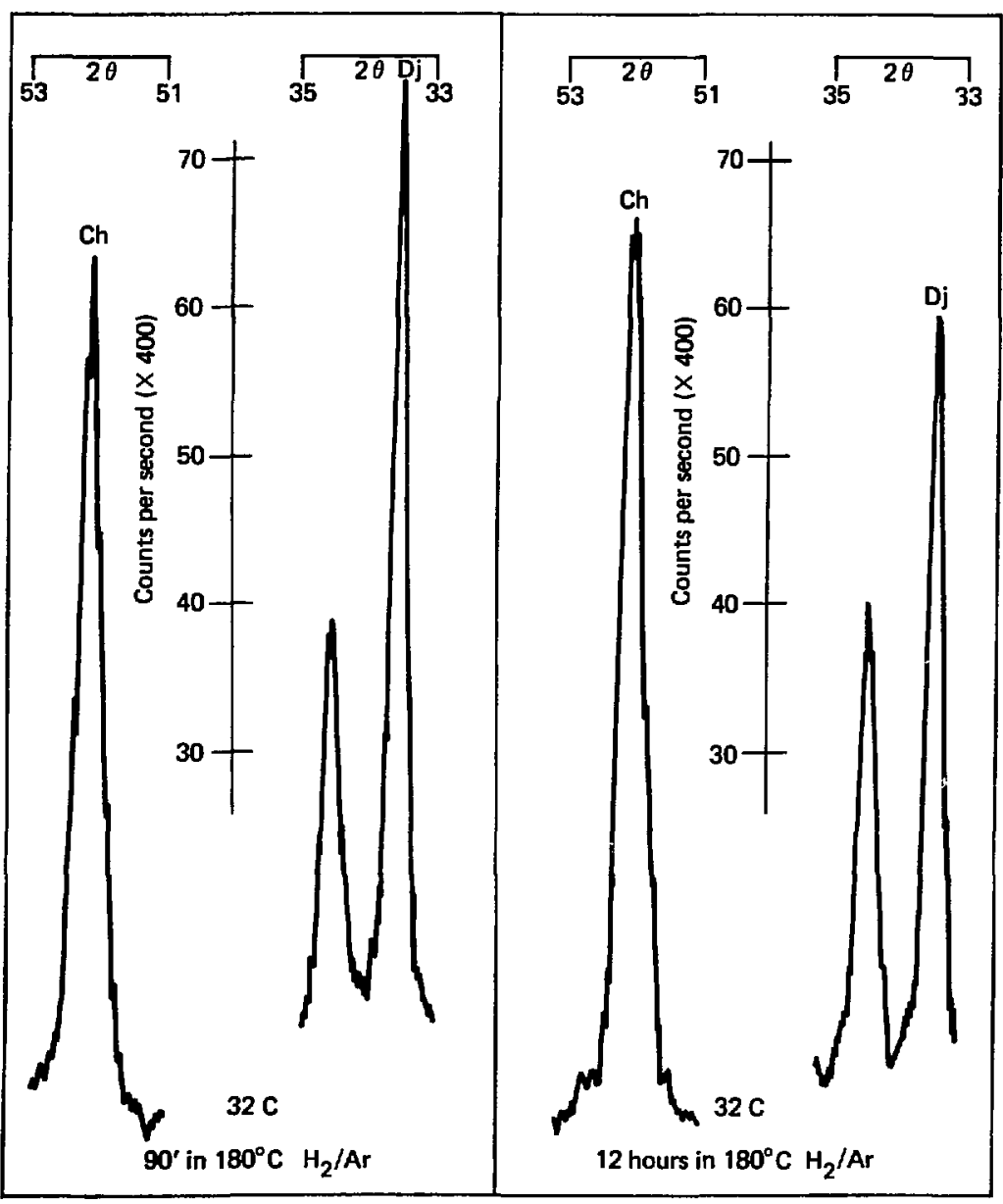

Figure 54. Phase change due to extend $\mathrm{H}_{2}$ /Ar heat treatment. 
The initial degradation observed in both environments is probably due to an oxidation process. Oxidation in $\mathrm{H}_{2} / \mathrm{Ar}$ may be the result of oxygen gettered on the film surface or in the dense network of grain boundaries, either during sputtering or upon exposure to air. This is a fast but self-limiting surface reaction. The higher concentration of oxygen in the air furnace allowed the process to reach its equilibrium faster. The oxidation process is completed at the expense of the $\mathrm{Cu}_{2} \mathrm{~S}$ which is converted to a more sulfur-rich djurleite.

A parallel process that may be taking place involves the loss of sulfur from the solid to vapor phase. The process is responsible for restoring the average composition of the films to their initial value or improving it beyond that state. This is a much slower process than that of extracting copper from $\mathrm{Cu}_{2} \mathrm{~S}$ with subsequent oxidation. Cook [19] found that heats of formation for $\mathrm{Cu}$ and $\mathrm{S}$ vacancies were $0.36 \mathrm{eV}$ and $2.7 \mathrm{eV}$, respectively. Yet, when the oxidation process reaches its saturation limit the sublimation of sulfur may become an important process, especially in the presence of $\mathrm{H}_{2}$. Kobayashi and Wagner ${ }^{[134]}$ have shown that removal of surface sulfur from $\mathrm{Cu}_{x} \mathrm{~s}$ by $\mathrm{H}_{2}$ is an efficient way to drive $\mathrm{Cu}_{x} \mathrm{~S}$ to stoichiometry. The high heat of formation of sulfur vacancies would seem to argue against this mechanisw. But, the $2.7 \mathrm{eV}$ heat of formation was measured in stoichiometric $\mathrm{Cu}_{2} \mathrm{~s}$. In highly degenerate films containing high concentrations of $\mathrm{Cu}$ vacancies there may be other conditions which may result in a significant reduction of the heat of formation. For example, highly strained local regions can be relaxed by creating sulfur vacancies with lower formation energies.

The generally accepted mechanism for regenerating $\mathrm{Cu}_{\mathrm{x}} \mathrm{S}$ is copper oxide ( $\mathrm{CuO}$ or $\mathrm{Cu}_{2}$ ) reduction by $\mathrm{H}_{2}$. Reduction of metal oxide by $\mathrm{H}_{2}$ is a common practice in metallurgy, but the reaction usually occurs at much higher temperatures than that used for heat treating the $\mathrm{Cu}_{x} \mathrm{~s}$. As yet, there is no conclusive evidence that oxide reduction by $H_{2}$ does take place in $\mathrm{Cu}_{\mathrm{x}} \mathrm{S}$. This mechanism is especially unlikely 
in the presence of air. Also, if copper oxide reduction is responsible for the regeneration of $\mathrm{Cu}_{\mathrm{x}} \mathrm{S}$ then the $\mathrm{H}_{2}$ treatments would restore not only the ratio of $\mathrm{Cu}_{2} \mathrm{~s}$ to djurleite but their absolute quantity as well. The $\mathrm{x}$-ray data in Figs. $32 \mathrm{a}$ and $54 \mathrm{~b}$ indicate a net loss of both $\mathrm{Cu}_{2} \mathrm{~S}$ and djurleite. 


\section{Temperature Effects on Optical Properties}

Measurement of the optical transmittance of $\mathrm{Cu}_{x} \mathrm{~S}$ at various temperatures offers another dimension with which to probe its band structire. It was also hoped that by reducing the temperature many of the spurious effects observed at room temperature may be reduced.

The transmittance at the highest $\left(295^{\circ} \mathrm{K}\right)$ and lowest $\left(81^{\circ} \mathrm{K}\right)$ temperatures are presented in Fig. 55. An inspection of the band edge characteristics for $\mathrm{T}=295^{\circ} \mathrm{K}$ and $\mathrm{T}=81^{\circ} \mathrm{K}$ demonstrates a point brought up in the discussion of the film \#19B. The knee and bump near $\lambda=950 \mathrm{~nm}$ are due to interference effects but may easily be misinterpreted as real features of the band structure. The knee at $\mathrm{T}=295^{\circ} \mathrm{K}$ became a bump at $\mathrm{T}=81^{\circ} \mathrm{K}$ as the absorbance decreases to the point that it cannot offset the contributions of positive interference.

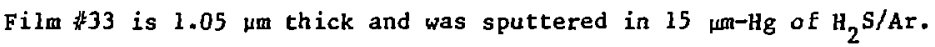
The absorption spectra and indices of refraction at $\mathrm{T}=81^{\circ} \mathrm{K}$ and $295^{\circ} \mathrm{K}$ are presented in Fig. 56. The index of refraction at $\mathrm{T}=81^{\circ} \mathrm{K}$ was determined from the transmittance extrema since a direct measurement of the reflectance of the film was not possible when it was mounted inside the transmission dewar. The indices of refraction at intermediate temperatures were interpolated from the two extrema values.

Plots of $\alpha^{1 / 2}$ and $\alpha^{2}$ versus hv at various temperatures are shown in Figs. 56 and 57, respectively. Both the direct and indirect gaps decreased with increasing temperature. The bandgap temperature coefficient $\left(I \mathrm{dE} g / \mathrm{dT} / \approx 10^{-4} \mathrm{eV} /{ }^{\circ} \mathrm{K}\right)$ are comparable to that of many semiconductors. $[39,84]$ The temperature coefficient for the direct gap remains fairly constant with increasing temperarure while that of the indirect gap increases with temperatures. The former is probably closer to the true value. The increase in $I d E / d T /$ with increasing temperature in the indirect gap is due to the increase in phonon population with increasing temperatures. The phonon population has no effect on direct transitions but is an essential 


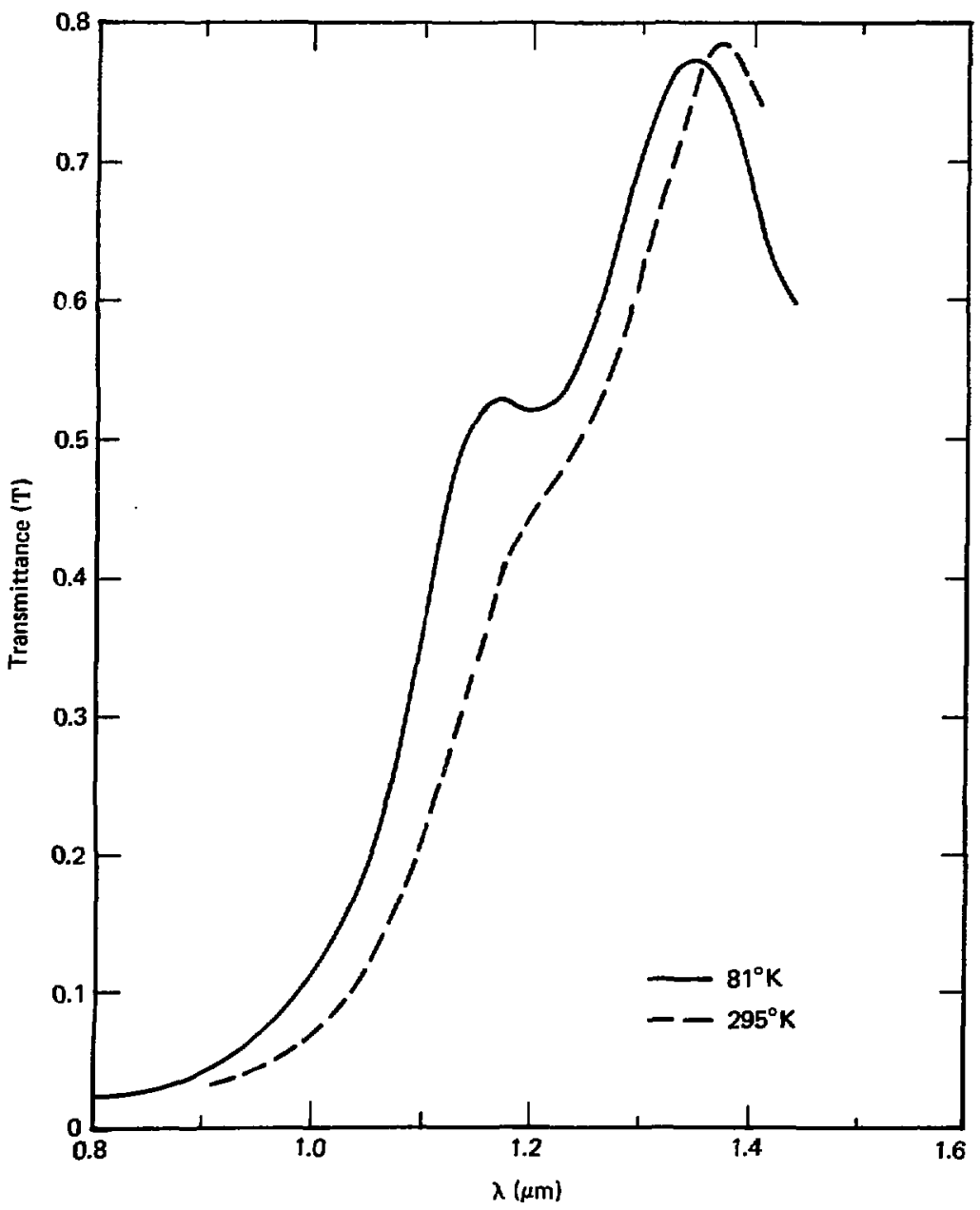

Figure 55. Transmittance spectra of high stoichiometry $\mathrm{Cu}_{x} \mathrm{~S}$ at $89^{\circ}$ and $295^{\circ} \mathrm{K}$. 


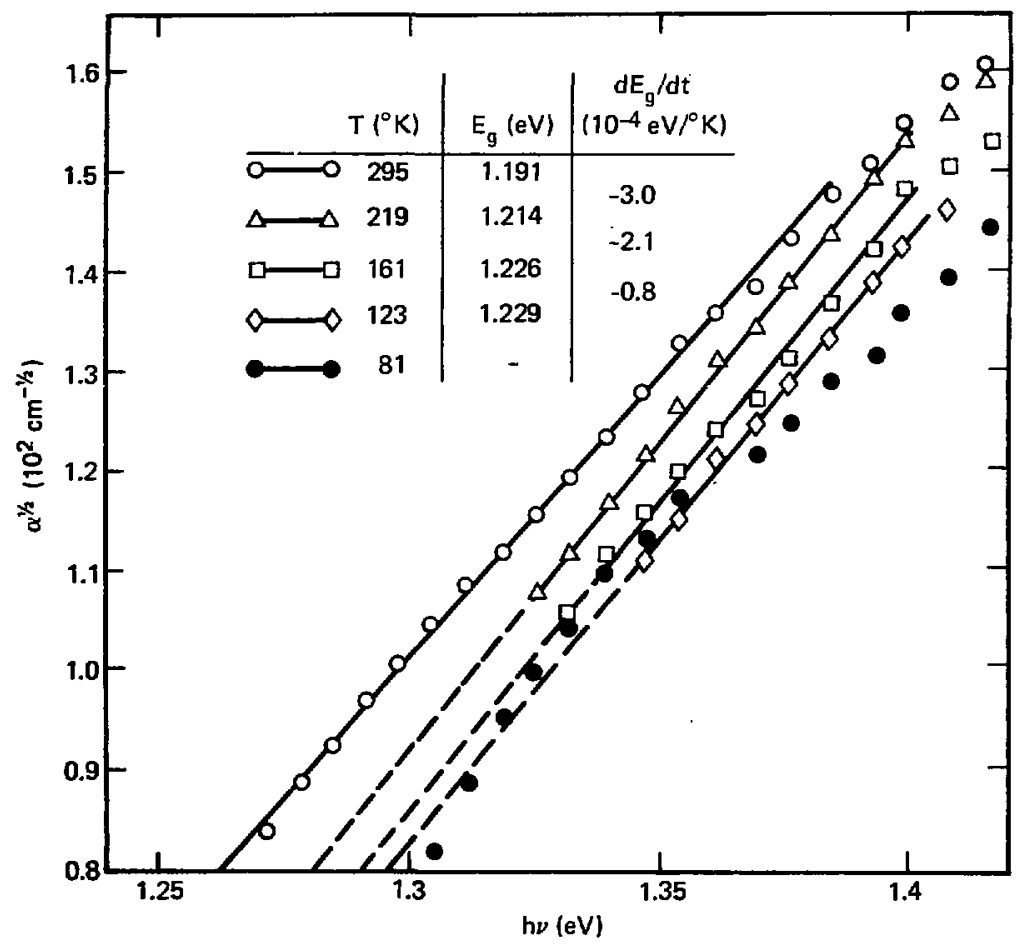

Figure 56. Variations in indirect gap with temperature. 
$-136-$

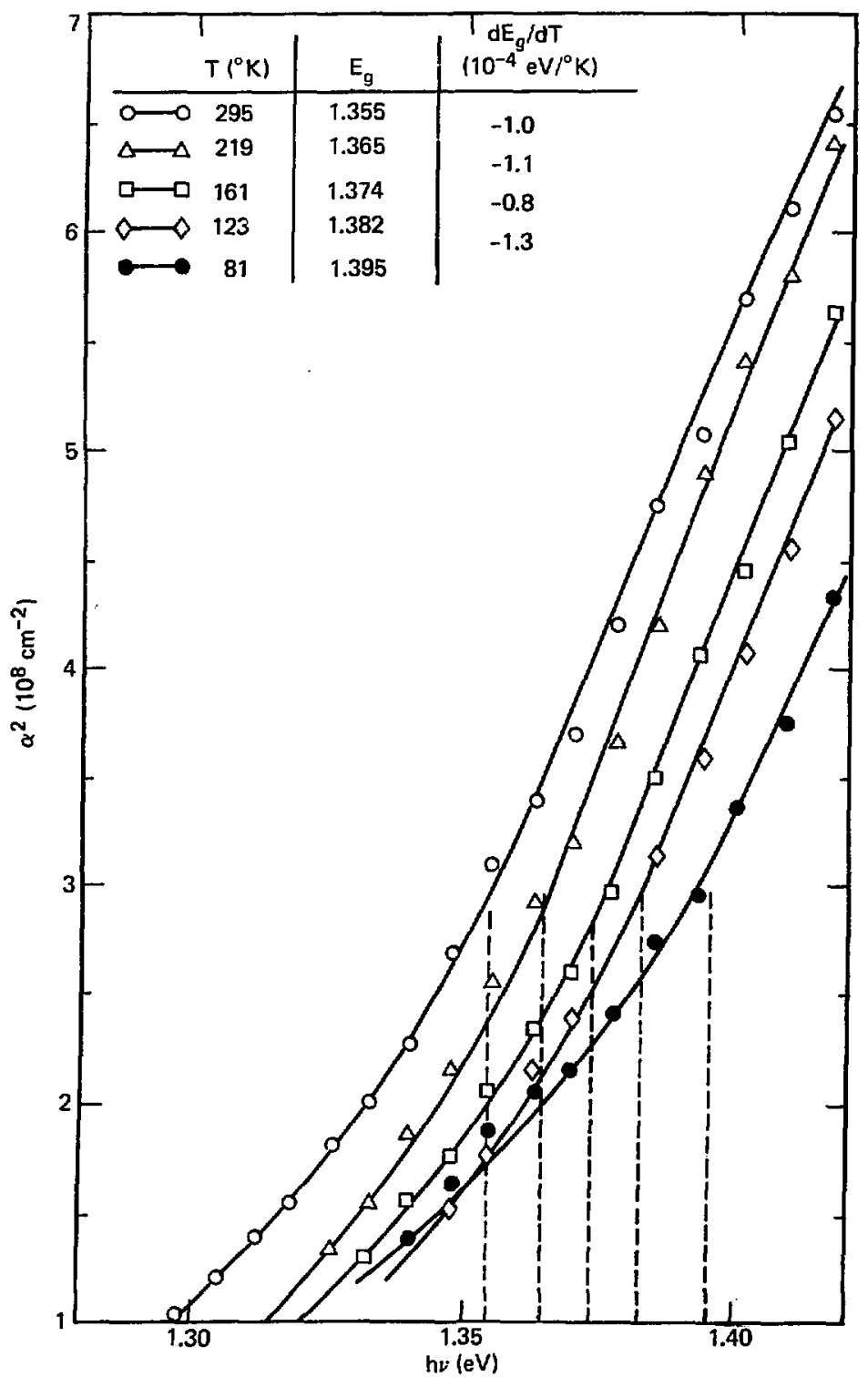

Figure 57. Variations in direct gap with temperature. 
factor for indirect transitions. These observations of fer further support for the existence of an indirect gap.

A comparative study was made on a more degenerate film. Film 非6A is $1.1 \mu \mathrm{m}$ and was sputtered in $20 \mu \mathrm{m}-\mathrm{Hg}$ of $\mathrm{H}_{2} / \mathrm{Ar}$. Its transmittance spectra at $\mathrm{T}=81^{\circ} \mathrm{K}$ and $289^{\circ} \mathrm{K}$ are shown in $\mathrm{Fig} .58$ and the graphic analysis of the bandgaps in Fig. 59. The bandgap temperature coefficient is still on the order of $10^{-4} \mathrm{eV} /{ }^{\circ} \mathrm{K}$. The interesting result of the experiment is the higher long wavelength absorption at 1 iquid nitrogen temperature when compared with that at room temperature. One would have expected the reverse to be true since there are more free carriers at the higher temperature. It appears that the long wavelength absorption is not entirely due to intra-band transitions. A major portion of the absorption may be due to transitions between the normal allowed bands and one or more broad impurity bands within the gap. A sketch of a possible distribution of states is shown in Figs. 60a and b. The density of states, $g(E)$, is assumed unchanged for simplicity. At room temperature the Fermi distribution function is fairly broad so that most of the localized states are partially filled. At liquid nitrogen temperatures the distribution function is much sharper. There are more filled states at lower energies and empty states at higher energies. Subgap photons will then be able to excite more electrons because more states are participating at the beginning and end of the transitions.

The contribution of chese subgap transitions may be responsible for the inconsistent conclusions on the dominant room temperature scattering mechanism. The analysis of Hall mobility the dominant mechanism is polar optical phonon scattering, but the $\mathrm{E}^{-3 / 2}$ dependence of tine long wavelength absorption characteristics suggested acoustic phonon scattering. The density of states of the impurity band may be such that shorter subgap wavelength photons are absorbed more readily. If the long wavelength absorption spectra of degenerate $\mathrm{Cu}_{x} \mathrm{~s}$ films are corrected for this contribution the true $\alpha_{f}$ may have a steeper dependence on energy and more consistent with polar optical phonon scattering. 


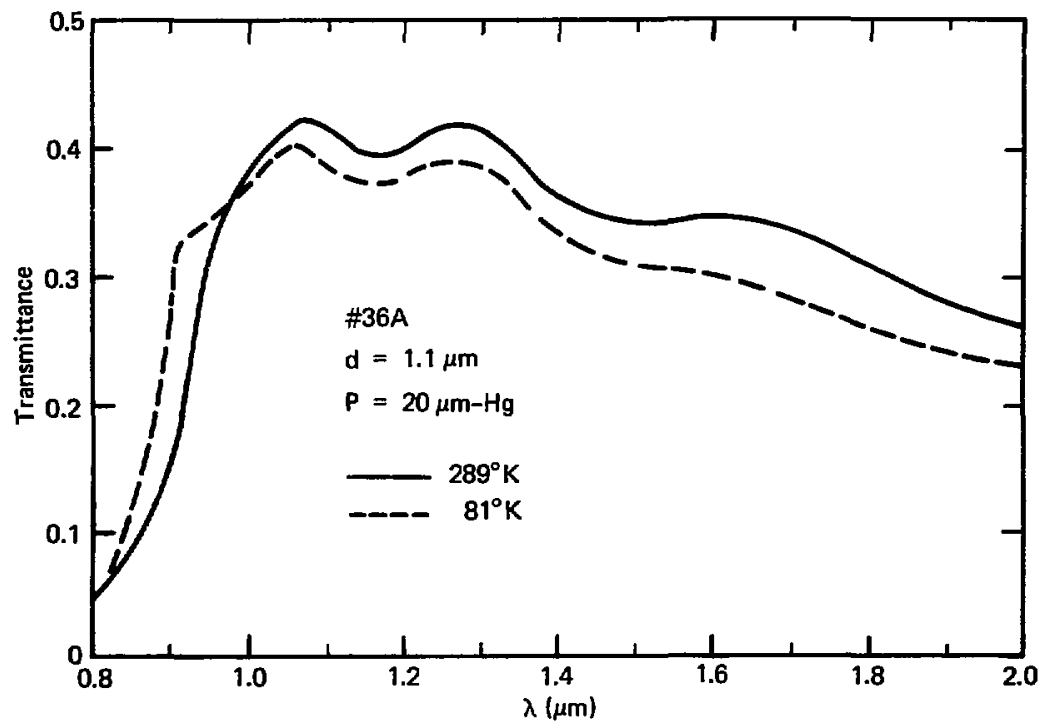

Figure 58. Transmittance spectra of degenerate $\mathrm{Cu}_{\mathrm{x}} \mathrm{S}$ film at $81^{\circ} \mathrm{K}$ and $289^{\circ} \mathrm{K}$. 


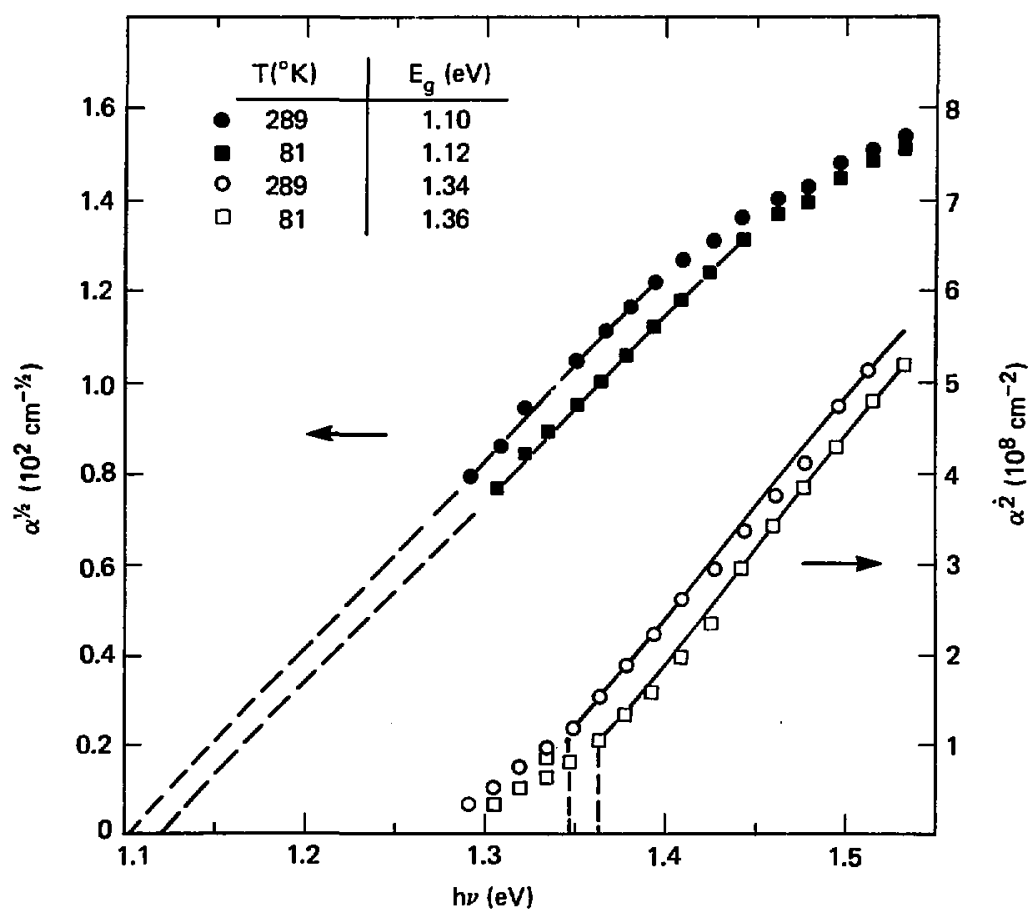

Figure 59. Variations in bandgaps of degenerate $\mathrm{Cu}_{x} \mathrm{~S}$ films with temperature. 

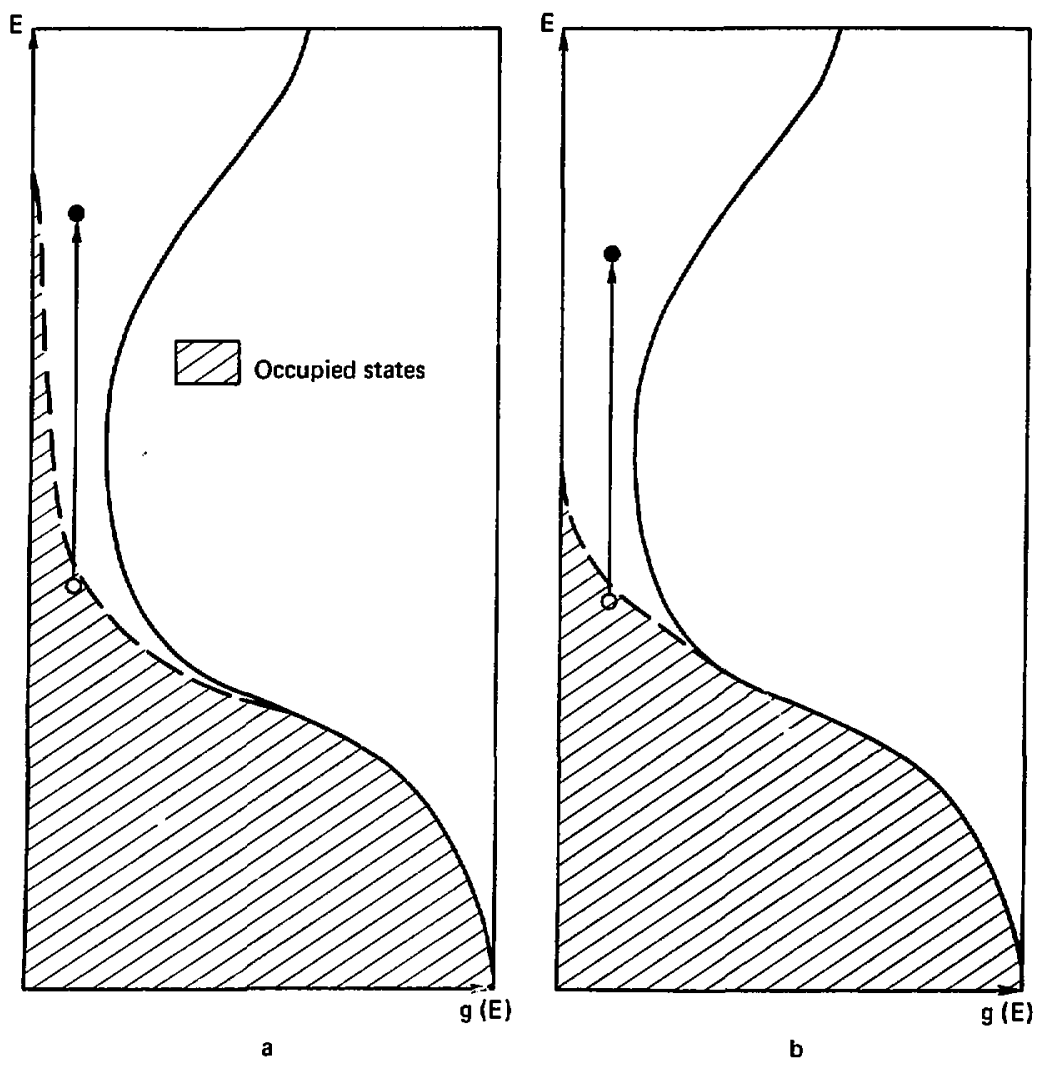

Figure 60. Density and occupancy of states at $289^{\circ} \mathrm{K}$ (a) and $81^{\circ} \mathrm{K}$ (b). 
VI. Summary and Conclusions

The primary objective of this research was to characterize thin $\mathrm{Cu}_{\mathrm{x}} \mathrm{S}$ films prepared by a reactive sputtering process in terms of their optical and electronic transport properties. The underlying 1 term objectives were to demonstrate the potential of the material and the reactive sputtering process for making efficient solar cells. Significant gains toward these goals were achieved in the course of the research. Specific results regarding the relevant properties of the $\mathrm{Cu}_{x} \mathrm{~S}$ and the effects of certain processing procedures are summarized and the implications of the results highlighted.

The films were prepared by sputtering copper in an $\mathrm{H}_{2} \mathrm{~S} / \mathrm{Ar}$ gas mixture. The Ar provided the ions for sputtering while the $\mathrm{H}_{2} \mathrm{~S}$ supplied the sulfur to react with sputtered $\mathrm{Cu}$ to form $\mathrm{Cu}_{x} \mathrm{~s}$ on a glass substrate. The film thicknesses were on the order of $1 \mu \mathrm{m}$. This thickness was chosen to be optimal for making the relevant measurements. It was sufficiently thick to remove most of the optical interference effects in the critical band edge region and to obtain resolvable $x$-ray diffraction patterns. At the same time it was thin enough to resemble practical films in $\mathrm{Cu} x \mathrm{~s} / \mathrm{CdS}$ solar cells.

High stoichiometry films can be prepared in a lean $\mathrm{H}_{2} \mathrm{~S} / \mathrm{Ar}$ gas pressure of 10-15 $\mu \mathrm{m}-\mathrm{Hg}$ with a corresponding $\mathrm{H}_{2} \mathrm{~S}$ partial pressure of 0.7-1.0 $\mu \mathrm{m}-\mathrm{Hg}$. The lower pressure samples contained excess copper in the form of cone shaped precipitates. The optical properties of the films, with and without excess copper, are very similar. Free carrier absorption is nearly undetectable. With increasing sputter gas pressures to as high as $30 \mu \mathrm{m}-\mathrm{Hg}$ free carrier absorption increased to a level comparable to the intrinsic absorption. 
Analysis of the band edge absorption characteristics resulted in an indirect gap at $1.15( \pm .05) \mathrm{eV}$ and a direct gap at $1.30( \pm .05)$ eV for all the films. This is contrary to the models proposed by several earlier studies $[29,32,131]$ which asserted that significant shifts in the effective bandgaps as much as $0.5 \mathrm{eV}$ can occur as $\mathrm{Cu}_{2} \mathrm{~S}$ degenerated to djurleite. On the other hand the fact that the two types of gaps are so close together may explain why $\mathrm{Cu}_{\mathrm{x}} \mathrm{S}$ have the optical characteristics of both a direct and indirect gap material. The high absorbance of the indirect gap material is due to the contributions of the direct transitions with threshold energies very close to the minimum gap. The main effect of higher sputter gas pressures on intrinsic absorption is to reduce short wavelength absorbance by perturbing the band structure so as to reduce the density of states or effective mass at higher energies.

The room temperature resistivity of the films 1 ie between 0.1 to $1.0 \mathrm{o}-\mathrm{cm}$. The results of Hall effect measurements indicated that impurity banding was responsible for the $10^{17}$ to $10^{20} \mathrm{~cm}^{-3}$ hole concentrations. The dominant scattering mechanisms are ionized impurity scattering at low temperatures $\left(\mathrm{T}<100^{\circ} \mathrm{K}\right)$ and polar optical phonon scattering at higher temperatures $\left(T>150^{\circ} \mathrm{K}\right.$ ). Increasing the sputtering gas pressure increased the hole concentration, most likely by increasing the density of $\mathrm{Cu}$ vacancies. The higher impurity concentration reduced the low temperature mobility. Room temperature mobility can also be affected by ionized impurity scattering when the impurity concentration is on the order of $10^{20} \mathrm{~cm}^{-3}$. But decreases in mobility in the higher temperature range is mainly due to an increase in polaron effective mass or stronger coupling with optical phonons resulting from the higher polarizability of the more degenerate phases.

The effect of air heat treatment on the films is similar to that of increasing the sputtering gas pressure. Degradation takes place very rapidly and saturates within the first five minutes. This corresponds to an increase in djurleite accompanied by a decrease in $\mathrm{Cu}_{2} \mathrm{~S}$. The conversion probably is due to the oxidation of $\mathrm{Gu}$ at the surface and 
along the grain boundaries of the film. Extended heat treatment in air indicated that degenerate films can be restored by heating in even an oxidizing environment. The positive effects may be due to annealing of physical defects or the restoration of the bulk Cu-s ratio by the sublimation of excess sulfur from a highly strained structure.

The effects of heat treating in $\mathrm{H}_{2} / \mathrm{Ar}$ which is believed to be a reducing ambient is less conclusive. The tendency is to produce less degenerate films with a higher relative concentration of $\mathrm{Cu}_{2} \mathrm{~S}$ to djurleite. But short term degradation can also occur, probably as a result of oxidation by trapped oxygen.

The study of temperature effects on the optical properties indicated that the bandgap of $\mathrm{Cu}_{x} \mathrm{~S}$ behaves in a manner similar to many other semiconductors. Both the direct and indirect gaps shrink with increasing temperature with a temperature coefficient on the order of $10^{-4} \mathrm{eV} /{ }^{\circ} \mathrm{K}$. The effect on long wavelength absorption revealed a peculiarity of degenerate semiconductors. The absorption of subgap photons not only causes transitions of free carriers within the normal allowed bands but also between those bands and the pseudo-continuum of states located in the gap. Normal free carrier absorption should increase with temperature as the concentration of free carriers increases. But broadening of the distribution function at high temperatures actually reduces the transition probability involving localized states.

The implications of these results may be divided into two groups, those concerned with the basic properties of $\mathrm{Cu}_{x} \mathrm{~s}$ and those affecting the efficiencies of $\mathrm{Cu}_{\mathrm{x}} \mathrm{S} / \mathrm{CdS}$ cells. Under the former category the rigid band model originally proposed by Mulder is invalidated. Shifting the Fermi level by over $0.5 \mathrm{eV}$ with no change in the band structure is not reasonable. A more acceptable model must at least allow for changes in the shape of the band extrema with changes in chemical phase. Analysis of the band edge absorption characteristics indicated that the 
density of states in the conduction band decreased with increasing degeneracy. This corresponded to the curvature of the conduction band minimum increasing, or band tailing such as that shown in Fig. 21 . On the other hand, the increase in hole effective mass, based on the analysis of the Hall mobility of the majority carriers, suggests an increase in the density of states at the top of the valence band with increasing degeneracy. This can occur as a result of a decrease in the curvature of the valence band maximum with the possibility of a small $(<0.1 \mathrm{eV})$ Burstein-Moss shift. The fact that significant increases in $\mathrm{m}_{\mathrm{h}}^{*}$ can occur with an undetectable Burstein-Moss shift indicates that the valence band maximum in $\mathrm{Cu}_{\mathrm{x}} \mathrm{S}$ is very broad, or that the $\mathrm{m}_{\mathrm{h}}$ is very large. This may, in part, account for the relatively low mobility of the holes. The other factor contributing to low carrier mobility at room temperature is scattering by polar optical phonons. Both of these 1 imitations on mobility are intrinsic to $\mathrm{Cu}_{x} \mathrm{~S}$ so that there is little one can do through processing to improve the transport properties. The maximum hole mobility should be on the order of $10 \mathrm{~cm}^{2} / \mathrm{V}-\mathrm{sec}$ for $\mathrm{Cu}_{2} \mathrm{~S}$ in which impurity concentration is at a minimum. For the more degenerate phases the hole mobility is even lower because of the increasing concentration of impurity scattering centers.

In the second category $\mathrm{Cu}_{x} \mathrm{~s}$ appears to have the potentials of an ideal thin film solar cell material in spite of its poor transport properties. It is an indirect gap material with a -rect gap within $0.2 \mathrm{eV}$ of the thermal gap. The lifetimes of excess carriers in indirect gap materials tend to be longer than that of direct gap materials. The main obstacle to achieving long lifetimes in $\mathrm{Cu}_{x} \mathrm{~S}$ is the high concentration of native defects (i.e., Cu vacancies). The resulting band of localized states provide excellent recombination paths for photogenerated carriers. It is in this area of material fabrication that significant improvements to cell efficiencies can be achieved through improved processing techniques. On the other hand, the low direct gap makes $\mathrm{Cu}_{\mathrm{x}} \mathrm{S}$ highly absorbent for most of the useful solar 
sirectrum. The high absorption coefficient moderates the negative effects of low nobility and short lifetime by reducing the oistance in $\mathrm{Cu}_{x} \mathrm{~s}$ through which the excess carriers have to be transported.

Finally, if $\mathrm{Cu}_{x} \mathrm{~S}$ is deposited in an isolated form as was done in this study, then it is possible to optimize its property by manipulating deposition parameters or by past deposition heat treatments prior to forming a solar cell with CdS. This approach avoids many effects on the CdS and the junction region that can occur if the same processing procedures are performed on a completed cell. Since $\mathrm{Cu}_{x} \mathrm{~S}$ is the active component of the $\mathrm{Cu}_{x} \mathrm{~S} / \mathrm{CdS}$ cell the additional degree of freedom afforded by this approach can have a major impact on improving the efficiency of the cell. 


\section{APPENDIX I: Transmittance and Reflectance}

of a Multi-layer Optical Medium

For a system of multiple parallel optical layers, Fig. I-1, the aroplitude of the electric field vector $(\rightarrow)$ in successive layers are given by a matrix relationship

$$
\left[\begin{array}{c}
\mathscr{E}_{\mathrm{m}-1}^{+} \\
\mathscr{E}_{\mathrm{m}-1}^{-}
\end{array}\right]=\frac{\mathrm{M}_{\mathrm{m}}}{\mathrm{t}_{\mathrm{m}}}\left[\begin{array}{l}
\mathscr{E}_{\mathrm{m}}^{+} \\
\mathscr{E}_{\mathrm{m}}^{-}
\end{array}\right]=\frac{1}{\mathrm{t}_{\mathrm{m}}}\left[\begin{array}{ll}
\mathrm{e}^{\mathrm{i} \delta_{\mathrm{m}-1}} & \mathrm{r}_{\mathrm{m}} \mathrm{e}^{\mathrm{i} \delta_{\mathrm{m}-1}} \\
\mathrm{r}_{\mathrm{m}} \mathrm{e}^{-i \delta_{\mathrm{m}-1}} & \mathrm{e}^{-\mathrm{i} \delta_{m-1}}
\end{array}\right]\left[\begin{array}{l}
\mathscr{E}_{\mathrm{m}}^{+} \\
\mathscr{E}_{\mathrm{m}}^{-}
\end{array}\right]
$$

where $\mathscr{E}_{\mathrm{m}-1}^{+}$and $\mathscr{E}_{\mathrm{m}-1}^{-}$are the amplitudes of the incident and reflected field vector in the $(m-1)$ th layer respectively,

$\mathscr{E}_{\mathrm{m}}^{+}$and $\mathscr{E}_{\mathrm{m}}^{-}$are the corresponding amplitudes in the adjacent $\mathrm{m}^{\text {th }}$ layer, immediately following the incerface.

$\tau_{m}$ is the Fresnel reflection coefficient of the interface between the two layers.

$t_{m}$ is the Fresnel transmission coefficent of the interface. $\delta_{m-1}$ is the complex phase change in traversing the $(m-1) \frac{\text { th }}{h}$ layers.

The complex phase change is related to the complex index of refraction (N)

$$
\delta_{m}=2 \pi N_{m} d / \lambda=2 \pi\left(\underline{n}_{m}-i \underline{k}_{m}\right) d_{m} / \lambda
$$

where $n_{m}$ and $k_{m}$ are the real and imaginary parts of $N_{m}$

$d_{m}$ is the layer chickness

$\lambda$ is the wavelength of 1 ight. 


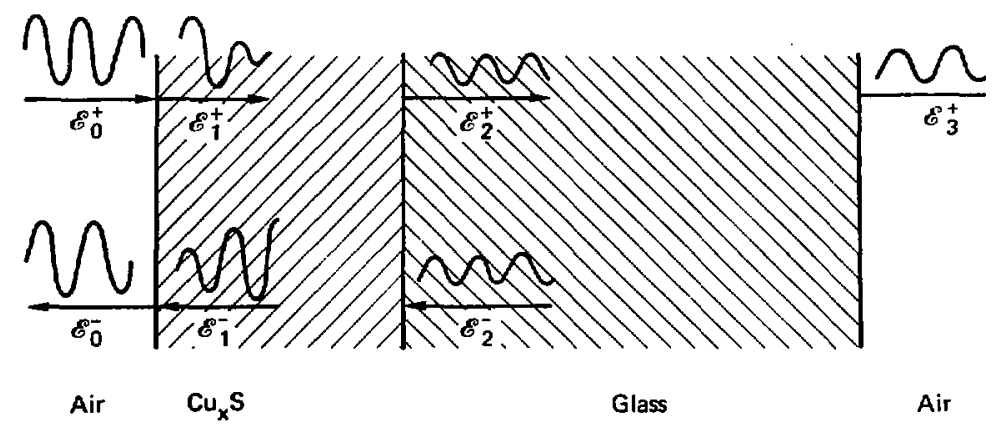

Figure 61. Light propagation in multi-layer medium. 
Alcernately,

$$
\delta_{m}=\frac{\varphi_{m}}{2}-i \frac{a_{m} d}{2}
$$

where $\varphi_{\mathrm{m}} \equiv 4 \pi \mathrm{n}_{\mathrm{m}} \mathrm{d} / \lambda$ is the real phase shift,

$a_{m} \equiv 4 \pi k_{m} / \lambda$ is the absorption coefficient.

Light passing through an absorbing media will be attentuated by $e^{-\alpha d} \mathrm{~m}$.

The two Fresnel coefficients are related to the indices of refraction in the following manner:

$$
\begin{aligned}
& r_{m}=g_{m}+i h_{m} \\
& t_{m}=\left(1+g_{m}\right)+i i_{m}
\end{aligned}
$$

where $\quad g_{m}=\frac{\left(\underline{n}_{-m-1}^{2}-\underline{n}_{-m}^{2}\right)+\left(k_{-m-1}^{2}-k_{-m}^{2}\right)}{\left(\underline{n}_{-n-1}+\underline{n}_{-m}\right)^{2}+\left(k_{-m-1}+k_{-m}\right)^{2}}$

$$
h_{m}=\frac{2\left(\underline{k}_{m} n_{m-1}-\underline{k}_{m-1} \underline{n}_{m}\right)}{\left(\underline{n}_{m-1}+\underline{n}_{m}\right)^{2}+\left(\underline{k}_{m-1}+\underline{k}_{m}\right)^{2}}
$$

For transparent layers $r_{1 n}$ and $t_{m}$ are reduced to the more familiar form

$$
\begin{aligned}
& r_{m}=\frac{n_{m-1}-\underline{n}_{m}}{\underline{n}_{m-1}+\underline{n}_{m}} \\
& r_{n}=1+r_{m}=\frac{2 \underline{n}_{m-1}}{\underline{n}_{n-1}+\frac{n}{m}}
\end{aligned}
$$


Transmittance $(T)$ and reflectance ( $R$ ) through the interface is

$$
\begin{aligned}
& T=\frac{\underline{n}_{m}}{\underline{n}_{m-1}}\left|t_{m}\right|^{2} \\
& R=\left|r_{m}\right|^{2}
\end{aligned}
$$

In the present study involving a $\mathrm{Cu}_{x} \mathrm{~S}$ film on a glass slide with air on both sides there will be three transition matrices corresponding to the three interfaces (see Fig. I.1). The incident $\left(\mathscr{E}_{0}\right)$ and the transmitted $\left(\mathscr{E}_{2}\right)$ field vectors are related by

$$
\left[\begin{array}{l}
\mathscr{E}_{0}^{+} \\
\mathscr{E}_{0}^{-}
\end{array}\right]=\frac{M_{1} M_{2} M_{3}}{t_{1} t_{2} t_{3}}\left[\begin{array}{l}
E_{2}^{+} \\
\mathscr{E}_{2}^{-}
\end{array}\right]
$$

with $\overrightarrow{\mathscr{E}_{2}}=0$, since there is no reflected component of the outgoing wave. The two matrices, $M_{1}$ and $M_{3}$, have a simple form

$$
M_{1}=\left[\begin{array}{ll}
1 & r_{1} \\
r_{1} & 1
\end{array}\right]
$$

and

$$
M_{3}=\left[\begin{array}{ll}
1 & r_{3} \\
r_{3} & 1
\end{array}\right]
$$

In both cases the phase shift consists only of the real part since $\underline{k}_{0}=\underline{k}_{2}=0$. There is no phase shift in crossing the front air/film interface (i.e., $\delta_{0}=\frac{\Phi_{0}}{2}=0$ ). In the case of $M_{3}$ the very large thickness of the glass $\left(d_{2} \ll \lambda\right)$ results in an average phase shift of $\delta_{2}=0$. The small angular divergence in the real beam, when considered over the large thickness, results in large optical path length differences. 
$-150-$

The transmittance and reflectance of this multilayer system is

$$
\begin{aligned}
& X=\frac{L}{A_{1} e^{\alpha d}+B_{1} \cos \varphi+C_{1} \sin \varphi+D_{1} e^{-\alpha d}} \\
& R=\frac{A_{2} e^{\alpha d}+B_{2} \cos \varphi+C_{1} \sin \varphi+D_{1} e^{-\alpha d}}{A_{1} e^{\alpha d}+B_{1} \cos \varphi+C_{1} \sin \varphi+D_{1} e^{-\alpha d}}
\end{aligned}
$$

where

$$
\begin{aligned}
& z=\left|t_{1}\right|^{2}\left|t_{2}\right|^{2}\left|t_{3}\right|^{2}= \\
& {\left[\left(1+g_{1}\right)^{2}+h_{1}{ }^{2}\right]\left[\left(1+g_{2}\right)^{2}+h_{2}^{2}\right]\left(1+g_{3}\right)^{2}} \\
& A_{1}=\left(1+g_{2} g_{3}\right)^{2}+h_{2}^{2} g_{3}^{2} \\
& B_{1}=2\left\{\left(1+g_{2} g_{3}\right) \quad\left[g_{1}\left(g_{2}+g_{3}\right)-h_{1} h_{2}\right]\right. \\
& \left.\left.+h_{2} g_{3} \quad\left[h_{1} h_{2}\left(g_{2}+g_{3}\right)+g_{1} h_{2}\right)\right]\right\} \\
& c_{1}=2\left\{\left(1+g_{2} g_{3}\right)\left[h_{1}\left(g_{2}+g_{3}\right)+g_{1} h_{2}\right]\right. \\
& \left.+h_{2} g_{3}\left[h_{1} h_{2}-g_{1}\left(g_{2}+g_{3}\right)\right]\right\} \\
& D_{1}=\left[g_{1}\left(g_{2}+g_{3}\right)-h_{1} h_{2}\right]^{2}+\left[g_{1} h_{2}+h_{1}\left(g_{2}+g_{3}\right)\right]^{2} \\
& A_{2}=\left[g_{1}+r_{3}\left(g_{1} g_{2}-h_{1} h_{2}\right)\right]^{2}+\left[h_{1}+r_{3}\left(h_{1} g_{2}+h_{2} g_{1}\right)\right]^{2} \\
& B_{2}=2\left\{h_{2}\left[h_{1}+g_{3}\left(h_{1} g_{2}-g_{1} h_{2}\right)\right]\right. \\
& \left.+\left(g_{2}+g_{3}\right)\left[g_{1}+g_{3}\left(g_{1} g_{2}-h_{1} h_{2}\right)\right]\right\}
\end{aligned}
$$


$c_{2}=2\left\{h_{2}\left[g_{2}+g_{3}\left(g_{1} g_{2}-h_{1} h_{2}\right)\right]\right.$

$\left.-\left(g_{2}+g_{3}\right)\left[h_{1}+g_{3}\left(h_{1} g_{2}+h_{2} g_{1}\right)\right]\right\}$

$D_{2}=\left(g_{2}+g_{3}\right)^{2}+h_{2}^{2}$

The dominant terms in the denominator which are responsible for the interference effects at long wavelengths are $A_{1}+B_{1} \cos \varphi$ when $a=0$. $B_{1}<0$ because the dominant term $g_{1}<0$. T is a maximum when $\varphi$ is an integer nultiple of $2 \pi$ and a minimum when it is $\pm \pi$ of that value because it is proportional to $\left(A_{1}-\left|B_{1}\right| \cos \varphi\right)^{-1}$.

The above equations reduce to Heavens ${ }^{\text {[118] }}$ results when the final substrate/air interface is ignored (i.e., $B_{3}=0$ ). The major effect of including the interface is seen in the replacement of $g_{2}$ by $g_{2}+g_{3}$. Since $g_{2}$ and $g_{3}$ are predominantly functions of the real indices of refraction and are of comparable value $\left(g_{2} \simeq .3, g_{3}=.2\right)$ the effective change is a replacement of the glass substrate with a significantly different transparent material. In fact that material is more like a vacuum (effective $n=1$ ) than glass.

The determination of $\varphi$ from real data is subject to large uncertainties. If

$$
\varphi+\Delta \varphi=4 \pi(n+\Delta n)(d+\Delta d) !(\lambda-\Delta \lambda)
$$

where $\Delta \varphi, \Delta n$, and $\Delta \lambda$ are the appropriate uncertainties, then

$$
\Delta \varphi=\frac{4 \pi n d}{\lambda}\left(\frac{\Delta \mathrm{n}}{\mathrm{n}}+\frac{\Delta \mathrm{d}}{\mathrm{d}}+\frac{\Delta \lambda}{\lambda}\right)
$$

With $\mathrm{d}=\lambda, \mathrm{n}=3$, and the combined relative uncertainty in the data is optimistically $6 \%$ then $\Delta \varphi \simeq \frac{3}{4} \pi$. Since the contribution of the interference terms changes from 0 to an extrema value within $\frac{\pi}{2}$, 
determining the contribution of the interference terms becomes a matter of probability. It is quite appropriate then to use a statistically average expression for $T$ in analyzing raw data,

$$
\langle T\rangle=\frac{1}{2 \pi} \int_{0}^{2 \pi} d \varphi=\frac{Z}{\left[\left(A_{1} e^{\alpha d}+D_{1} e^{-\alpha d}\right)^{2}-B_{1}^{2}\right]^{1 / 2}}(I-28)
$$

in which the fact that $\left|B_{1}\right| \gg C_{1}$ was used for simplification. 


\section{APPENDIX II: Direct Optical Transitions}

Transitions between a valence band maximum and conduction minimum wich are located at the same point in the Brillouin zone are "direct" transitions. The derivation presented here are for $\vec{k}=0$. It is based on time dependent perturbation theory. [135]

The quantum mechanical Hamiltonian for an electron in the presence of an electromagnetic field may be expressed by

$$
\mathrm{H}=\mathrm{H}_{\mathrm{o}}+\mathrm{H}_{\mathrm{l}}
$$

where $H_{0}$ is the unperturbed Hamiltonian;

$H_{1}=-\frac{i q h}{m_{0}} \vec{A} \cdot \nabla$ is the perturbation operator;

$\vec{A}=\vec{A}_{0} e^{i(\vec{R} \cdot \vec{r}-\omega t)}+($ complex conjugate) is the vector potential;

$q$ and $m_{o}$ are the charge and free mass of the electron, respectively;

$\vec{K}$ and $\omega$ are the wave vector and angular velocity of the perturbing field, respectively;

$\vec{r}$ is the spatial coordinate;

$t$ is time;

$n$ is Planck's constant;

and $c$ is the speed of light.

For an electron in a crystalline solid the eigenfunction of $\mathrm{H}_{0}$ is

$$
\Psi(\vec{r}, t)=e^{-i E(k) \vec{t} / r} y(\vec{r})
$$

where $\mathrm{E}$ is the energy eigenvalue 
and $\vec{k}$ is the wave vector of the electron.

The spatial dependent part is a Bloch type function

$$
\Psi(\vec{r})=e^{i \vec{k} \cdot \vec{r}} u(\vec{k}, \vec{r})
$$

where $u(\vec{k}, \vec{r})$ has the periodicity of the lattice.

The transition probability from state $i$ to state $f$ is

$$
P_{F I}=\hbar^{-2}\left|\iint_{F}^{*}(\vec{r}, t) H, \psi_{I}(\stackrel{+}{r}, t) d d^{+} d t\right|^{2}
$$

Integration with time gives

$$
P_{F I}=\left(\frac{q A_{0}}{m c}\right)^{2}\left[\frac{2 \sin \frac{\omega-\omega_{0}}{2}}{\omega_{0}-\omega}\right]_{\left|M_{F I}\right|^{2}}^{2}
$$

where

$$
\begin{aligned}
\omega_{0} & =\left(E_{F}-E_{I}\right) / \hbar \\
\psi_{F I} & =\int \Psi_{F}^{*}(\vec{r}) e^{i \vec{K} \cdot \vec{r}} \nabla \Psi_{I}(\vec{r}) d \vec{r} \\
& \approx e^{i \vec{K} \cdot \vec{r}} \int \Psi_{F}^{*} \nabla \Psi_{I} d \vec{r} .
\end{aligned}
$$

The $e^{i \vec{k} \cdot \vec{r}}$ factor was taken out of the integral on the approximation that the wavelength of che perturbing field is much larger than the atomic dimensions over which the rest of the integration is significantly non-zero.

Eqs. (IT.5) and (II.6) encompass two selection rules. First,

$$
\frac{\sin \left(\frac{\omega-\omega_{0}}{2}\right) t}{\omega_{0}-\omega} \text { peaks sharply at } \omega=\omega_{0} \text {. }
$$


This is synonymous with the principle of the conservation of energy. This term is due to the integration of $\mathrm{A}_{0} \mathrm{e}^{\mathrm{i}(\overrightarrow{\mathrm{K}} \cdot \overrightarrow{\mathrm{r}}-\omega \mathrm{L})}$ corresponding to the absorption of a photon. If the transition involves emission of a photon then the dominant term is due to the complex conjugate $A_{0} e^{i(\vec{K} \cdot \vec{r}+\omega t)}$ with $\omega+\omega_{0}$ replacing $\omega-\omega_{0}$ in Eq. (II.5). The second selection rule is contained in $\mathrm{M}_{\mathrm{fi}}$ which vanishes unless

$$
\vec{k}_{F}-\vec{k}_{I}-\vec{k} \simeq \vec{k}_{F}-\vec{k}_{I}=\vec{\imath}
$$

where $\vec{\ell}$ is a translation vector in reciprocal lattice space. For small perturbations the transitions are within the same Brillouin zone, or $\overrightarrow{\mathbb{L}}=0$. This is equivalent to the conservation of momentum.

The total transition probability with $w$ must include all possible $\vec{k}$.

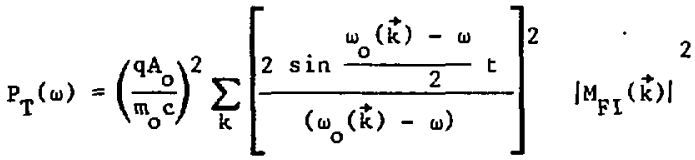

$$
\begin{aligned}
& =\left(\frac{\mathrm{qA}_{0}}{\mathrm{~m}_{\mathrm{o}} \mathrm{c}}\right)^{2} \int\left[\frac{2 \sin \frac{\omega_{0}-\omega}{2}}{\omega_{0}-\omega}\right]^{2} N(\vec{k}) \mathrm{dk} \mid \mathrm{M}_{\mathrm{FI} \mid}{ }^{2}
\end{aligned}
$$

Assuming parabolic energy surfaces, the creation of an electron-hole pair will involve initial and final energies of

$$
\begin{aligned}
& E_{I}=E_{v}-\frac{\left(\pi k_{i}\right)^{2}}{2 m_{h}^{\frac{1}{h}}} \\
& E_{F}=E_{c}+\frac{\left(\pi k_{f}\right)^{2}}{2 m_{e}^{*}}
\end{aligned}
$$


where $E_{*}$ and $E_{*}$ are the valence and conduction band edges, respectively, and $m_{h}^{*}$ and $m_{e}^{*}$ are the hole and electron effective masses, respectively. Invoking the second selection rule, or conservation of momentum,

$k_{1}=k_{F}=k=\frac{2 \mu^{*}\left(n \omega_{o}-E_{g}\right)^{1 / 2}}{\hbar}$

where $\mu^{*-1}=m_{h}^{*-1}+m_{c}^{*-1}$

and $E_{g}=E_{c}-E_{v}$ is the band gap.

The density of states in $k$-space with unit volume $(2 \pi)^{3}$ and allowing for two spins is

$$
\begin{aligned}
\dot{N}(\vec{k}) d \vec{k} & =\frac{2\left(4 \pi k^{2} d k\right)}{(2 \pi)^{3}}=\frac{k^{2} d k}{\pi^{2}}, \\
& =\frac{\left(2 \mu^{*}\right)^{3 / 2}\left(\hbar \omega_{o}-E_{g}\right)^{1 / 2}}{2 \pi^{2} \hbar^{2}} d \omega_{0}
\end{aligned}
$$

Eq. (II.9) becomes

$$
\begin{aligned}
P_{T}(\omega)=\left(\frac{q A_{0}}{m_{0} c}\right)^{2} \frac{\left(2 \mu^{*}\right)^{3 / 2}}{2 \pi^{2} \hbar^{2}} \int_{0}^{\infty} & {\left[\frac{2 \sin \left(\frac{\omega_{0}-\omega}{2}\right) t}{\omega_{0}-\omega}\right]^{2} } \\
& x \cdot\left(\hbar \omega_{0}-E_{g}\right)^{1 / 2} d \omega_{o}\left|M_{F I}\right|^{2} .
\end{aligned}
$$

The facior ( $\left.\hbar \omega_{\circ}-E_{g}\right)^{1 / 2}$ can be taken outside the integral because of the sharp maximum of the integrand at $\omega_{0}=\omega$. The rest of the integration can be completed by an appropriate change of variable.

$$
P_{T}(\omega)=\left(\frac{q A_{o}}{m_{0} c}\right)^{2} \frac{\left(2 \mu^{*}\right)^{3 / 2}}{2 \pi h^{2}} t\left(\hbar \omega-E_{g}\right)^{1 / 2}\left|M_{F I}\right|^{2}
$$


The energy density is given by

$$
\begin{aligned}
I(\omega) & =\frac{\epsilon\left\langle\mathscr{E}^{2}\right\rangle}{4 \pi} \\
& =\frac{\varepsilon}{8 \pi}\left(\frac{\omega A_{0}}{c}\right)^{2}
\end{aligned}
$$

where $E$ is the optical dielectric constant;

$\mathscr{E}$ is the electric field.

The attenuation per unit distance in an absorbing medium is

$$
-\frac{d I}{d x}=\frac{\hbar \omega P(\omega) / 4 \pi}{t(c / \underline{n})}
$$

where $\underline{n}$ is the index of refraction.

The factor $P(\omega) / 4 \pi$ is the transition probability per solid angle or differential interaction cross-section while $c / \underline{n}$ is the phase velocity in the medium. The absorption coefficient for direct transitions is given by

$$
\begin{aligned}
a_{d} & =-\frac{1}{I} \frac{d I}{d x} \\
& =\frac{2(2 \mu)^{3 / 2}}{\pi \underline{n} c}\left(\frac{g}{m_{o}}\right)^{2} \frac{\left(\hbar \omega-E_{g}\right)^{1 / 2}}{\hbar \omega} \mid \frac{M}{\left.F I\right|^{2}}{ }^{2}
\end{aligned}
$$

It can be shown that [136]

$$
\left|M_{F I}\right|^{2} \simeq \frac{m_{0} w_{o}}{2}\left(1+\frac{m_{0}}{m_{h}^{*}}\right)
$$

Then,

$$
u_{d}=\frac{(2 \mu / m)^{3 / 2}}{\pi \underline{n} a_{o} E_{o}^{1 / 2}}\left(1+\frac{m_{o}}{m_{h}^{\frac{1}{2}}}\right)\left(\hbar \omega-E_{g}\right)^{1 / 2}
$$


where

$$
\begin{aligned}
& a_{0}=\frac{n^{2}}{m_{0} q^{2}} \text { is the Bohr radius } \\
& E_{0}=m_{0} c^{2} \text { is the electron rest mass energy equivalent. }
\end{aligned}
$$

with

$$
\begin{aligned}
& \mathrm{m}_{\mathrm{h}}^{*}=\mathrm{m}_{\mathrm{c}}^{*}=\mathrm{m}_{\mathrm{o}}=2 \mu^{*}, \\
& a_{\mathrm{d}}=4.2 \times 10^{4}\left(\mathrm{n}_{\omega}-\mathrm{E}_{\mathrm{g}}\right)^{1 / 2} \mathrm{~cm}^{-1} \\
& \quad \text { or } \\
& a_{\mathrm{d}}=10^{4} \mathrm{~cm}^{-1}
\end{aligned}
$$

near the threshold where the exciting monochromatic beam is within $k_{B} T$ of the bandgap. 


\section{APPENDIX III: Indirect Optical Transitions}

When the valence band and conduction band extremas are not located at the same point in the Brillouin zone, the interband transicion is an indirect one. It involves the absorption of a photon and the emission or absorption of a phonon. The transition probability of two quanta processes are much lower than that of the one quantum direct transition [56] and must be computed with second order perturbation terms. The transition probability is given by [137]

$$
P=\frac{2 \pi}{n} \sum_{i} \frac{\left|\mathrm{H}_{I M} \mathrm{H}_{M F}\right|^{2}}{\left(E_{i}-E_{0}\right)^{2}}
$$

where $I, M, F$ are the indices for the initial, intermediate and final states, respectively;

$\mathrm{H}_{I M}$ is the matrix element due to interactions with the radiation field;

$H_{M F}$ is that due to interactions with the phonons;

$N\left(E_{F}\right)$ is the density states with final energy $E_{F}$.

The matrix elements in Eq. (III.1) can be evaluated only if the spectrum of intermediate states are known. Lacking this information it is not possible to express the indirect absorption coefficient ( $a_{i}$ ) in any detail. But just as in the direct transition case the dependence of $a_{i}$ on the energy difference (tiw $-E_{g}$ ) can be determined from appropriace densities of states and distribution functions. The density of initial and final states are

$$
N\left(E_{I}\right)=\frac{1}{2 \pi^{2} \hbar^{3}}\left(2 m_{h}^{*}\right)^{3 / 2}+E_{I} 1^{1 / 2}
$$


and

$$
N\left(E_{F}\right)=\frac{1}{2 \pi^{2} \hbar^{3}}\left(2 m_{e}^{*}\right)^{3 / 2}\left(E_{F}-E_{g}\right)^{1 / 2} \text {, respectively. }
$$

For phonon absorption the appropriate distribution function is the Bose-Einstein distribution function

$$
f_{p}(\theta)=\left(e^{\theta / T}-1\right)^{-1}
$$

where $\theta$ is the characteristic temperature of the phonons.

The energy of the phonons is $k_{B} \theta$. The transition probability due to the absorption of a photon and a phonon is

$$
\begin{aligned}
P_{1}=A_{1} f_{P}\left(E_{P}\right) \int_{E_{F}=0}^{\infty} \int_{E_{I}=0}^{E_{g}-\hbar \omega-k_{B} \theta} & \left(E_{I}\right)^{1 / 2}\left(E_{F}-E_{g}\right)^{1 / 2} \\
& \times \delta\left(E_{F}-E_{I}-\hbar \omega-k_{B} \theta\right) d E_{I} d E_{F}
\end{aligned}
$$

where $A_{1}$ contains all the energy independent terms.

The delta function is required for energy conservation. Integrating with respect to $\mathrm{E}_{\mathrm{F}}$

$$
P_{1}=A_{1} F_{p} \int_{0}^{E E^{-\hbar \omega-k_{B} \theta}}\left(E_{I}\right)^{1 / 2}\left(E_{i}-E_{g}+\hbar \omega+k_{B} \theta\right)^{1 / 2} d E_{i}
$$


A change of variables results in

$$
\begin{aligned}
P_{l} & =2 A_{L} f_{p} \int_{0}^{\left(E_{g}-\hbar \omega-k_{B} \theta\right) / 2}\left(u^{2}-u_{0}^{2}\right) d u \\
& =A_{1} f_{p} \frac{\pi_{0}}{8} \quad\left(E_{g}-\hbar_{\omega}-k_{B} \theta\right)^{2}
\end{aligned}
$$

where $u \equiv E_{i}+\frac{l}{2}\left(E_{g}-\hbar_{\omega}-k_{B} \theta\right)$

and

$$
u_{0}=\frac{l}{2}\left(E_{g}-\hbar \omega-k_{B} \theta\right)
$$

For phonon emission the appropriace distribution function is

$$
1+f_{p}=\left(1-e^{-\theta / T}\right)^{-1}
$$

The forms of the distribution functions in Eqs. (III.4) and (III.9) are due to the quantization of bosons. ${ }^{[138]}$ The derivation of the transition probability due to the absorption of a photon and emissi a of a phonon is similar to that used to obtain Eq. (III.8). The total absorption coefficient for indirect transitions is then

$$
\begin{aligned}
a_{i}= & ,\left[\frac{\left(\hbar \omega+k_{B} \theta-E_{g}\right)^{2}}{e^{\theta / T}-1} S\left(\hbar \omega+k_{B} \theta-E_{g}\right)\right. \\
& \left.+\frac{\left(\hbar \omega-k_{B} \theta-E_{g}\right)^{2}}{1-e^{-\theta / T}} S\left(\hbar \omega-k_{B} \theta-E_{g}\right)\right]
\end{aligned}
$$

where $S(x)=0$ for $x<0$

$$
=1 \text { for } x \geq 0
$$

and A' contains all the energy independent terms. 
APPENDIX IV: Ionized Impurities Scattering

and the Relaxation Iime Approximacion

The steady szate Boltzman transport equation is [139]

$$
\vec{v} \cdot \nabla_{\tau} f+\dot{\vec{k}} \nabla_{k} f=\left(\frac{\partial f}{\partial t}\right)_{c}
$$

where $f=f(\vec{r}, \vec{k})$ is the distribution function of particles in six-dimensional phase space,

$\vec{v}$ is the particle velocity,

$\underset{\mathrm{h}}{\overrightarrow{\mathrm{k}}} \quad$ is the particle acceleration due to external forces, $\left(\frac{\partial f}{\partial t}\right)_{c} \quad$ is the net rate of particles entering the phase volume element $(\vec{r}, \vec{k})$ due to collisions.

The flux of particles from $(\vec{r}, \vec{k})$ due to its velocity and acceleration is balanced by the flux into $(\vec{r}, \vec{k})$ resulting from collision processes. The collision term is given by

$$
\begin{aligned}
\left(\frac{\partial f}{\partial t}\right)_{c}=\int & \left\{s\left(\vec{k}^{\prime}, \vec{k}\right) f\left(\vec{k}^{\prime}\right)[1-f(\vec{k})\}\right. \\
& \left.-s\left(\vec{k}, \vec{k}^{\prime}\right) f(\vec{k})\left\{1-f\left(\vec{k}^{\prime}\right)\right]\right\} d \vec{k} .
\end{aligned}
$$

where $\left(\vec{k}^{\prime}, \vec{k}\right)$ is the probability per unit cime of a carrier being scattered from state $\left(\vec{k}^{\prime}\right)$ to state $(\vec{k})$,

$f\left(\vec{k}^{\prime}\right)$ is the probability chat the particle initially occupies state $\left(\vec{k}^{\prime}\right)$,

and $[1-f(\vec{k})]$ is the probability that state $(\vec{k})$ is unoccupied.

According to the principle of micruscopic reversibility $\{1+0\rfloor$

$$
s\left(\vec{k}^{\prime}, \vec{k}\right)=\left(\vec{k}, \vec{k}^{\prime}\right) \text {. }
$$


so,

$$
\left(\frac{\partial f}{\partial t}\right)_{c}=\int s\left[f\left(\vec{k}^{\prime}\right)-f(\vec{k})\right] d \vec{k} .
$$

For elastic collisions it is possible to define a relaxation time ( $\tau)$ by

$$
\left(\frac{\partial f}{\partial \tau}\right)_{c}=-\frac{\left(f-f_{o}\right)}{\tau}
$$

The distribution relaxes to its equilibrium $F_{0}$ exponentially in time via collisions with $\tau$ as its time constant.

When $\vec{k}^{\prime}$ and $\vec{k}$ differ only in direction the integration in Eq. (IV.4) need only be aken about a spherical shell in k-space. The distribution functions can be expanded about the equilibrium value $\left(f_{0}\right)[141]$

$$
f(\vec{k})=f_{0}(E)+k_{x} x(E)
$$

where $k_{x}$ is the projection along a defined $x$-direction.

$$
\begin{aligned}
& x(E) \text { is a small function dependent only on the magnitude of } \vec{k} \\
& \text { through } \mathrm{E} \text {. }
\end{aligned}
$$

Thèn,

$$
\begin{aligned}
f\left(\vec{k}^{\prime}\right) & -f(\vec{f})=x(E)\left(k_{x}^{\prime}-k_{x}\right) \\
& =\left(\frac{f-f_{0}}{k_{x}}\right)\left(k_{x}^{\prime}-k_{x}\right) \\
& =-\left(f-f_{0}\right)(1-\cos \theta)
\end{aligned}
$$

where the $x$-axis is defined along $\vec{k}$

$\theta$ is the scattering angle between $k$ and $k$ '?

The relaxation time can then be defined by

$$
\tau^{-1}=\int s(1-\cos \theta) \mathrm{d} \Omega \text {. }
$$


From quantum mechanical scattering theory $142 \mid$

$$
s=N_{i} \frac{W}{4 \pi}
$$

where $\quad W=\frac{2 \pi}{k}|\langle\vec{k} \cdot|V(r)| \vec{k}\rangle|^{2} \rho(E)$

is Fermi's Golden Rule for the transition rate,

$V(r)$ is the scattering potential,

$\mathrm{N}_{\mathrm{i}}$ is the density of sattering centers,

$p(E)$ is the density of states.

Born's approximation can be used to calculate the matrix element

$$
\langle\vec{k},|v| \vec{k}\rangle=\frac{4 \pi}{k} \int_{0}^{\infty} v(r) \sin (k r) r d r
$$

where $k=|\vec{k} \cdot-\vec{k}|=2 k$ sin $\frac{\theta}{2}$

$$
\mathbf{k}^{\prime}=\mathbf{k} \text { is assumed. }
$$

For scattering by ionized impurities with a screened coulomb potential

$$
v=\frac{z q^{2}}{e r} e^{-r / r_{0}}
$$

where $z$ is the number of charges (i.e., ion valence),

$\varepsilon$ is the dielectric constant,

$r_{0}$ is the screening radius.

The screening radius is given by [103]

$$
\begin{aligned}
& r_{0}=\left[\frac{1_{1}^{3}}{16 \pi^{2} q^{2}{ }^{3 / 2}\left(2 \pi k_{B} T\right)^{1 / 2} F_{-1 / 2}(\pi)}\right]^{1 / 2} \\
& =\frac{9.92 \times 10^{-8} 1 / 2}{T^{1 / 4}\left[\mathscr{F}_{-1 / 2}(n)\right]^{1 / 2}}\left(\frac{m_{0}}{m^{\frac{*}{*}}}\right)^{3 / 4} \text { in c.g.s. units (IV-14) }
\end{aligned}
$$


The Fermi-i)irac integral is of the form

$$
F_{\ell}(\pi)=\frac{1}{\ell !} \int_{0}^{\infty} \frac{x^{\ell} d x}{1+\exp (x-\pi)}
$$

with $\eta=E_{f} / k_{B} T$ as the reduced Fermi energy.

For a p-type semiconductor $n<\varphi$ if $E_{f}$ is above the valence band edge and $n>0$ if it is below. The Fermi energy is related to the carrier concentration by

$$
\mathscr{F}_{1 / 2}(n)=2.07 \times 10^{-16} \mathrm{pT}^{-3 / 2}\left(\frac{\mathrm{m}_{\mathrm{o}}}{\mathrm{m}^{2}}\right)^{3 / 2}
$$

A partial table of $\mathscr{S}_{-1 / 2}(n)$ and $\boldsymbol{F}_{1 / 2}(n)$ is reproduced in Table 3 from Dingle. [103]

Substituting Eq. (IV.12) into (IV.11) and integrating gives

$$
\langle\vec{k} \cdot|V| \vec{k}\rangle=\frac{4 \pi z q^{2} r_{o}^{2}}{\left(k^{2} r_{o}^{2}+1\right)}
$$

The density of states with spin conserved and assuming parabolic energy surfaces is

$$
p(E)=\frac{4 \pi}{h^{3}}(2 E)^{1 / 2} \mathrm{~m}^{\hbar^{3 / 2}}
$$

Combining the resulcs of Eqs. (IV.9) through (IV.18) into (IV.8)

$$
t^{-1}=A\left[\ln (1+B)-\frac{B}{1+B}\right]
$$

where $A=2 x N_{I}\left(\frac{z^{2}}{\varepsilon}\right)^{2} /(2 E)^{3 / 2} \mathrm{~m}^{* 1 / 2}$

and

$$
B=4 x^{2} r_{0}^{2}
$$


Transport parameters such as current density $(\mathrm{J})$, conductivity $(\sigma)$, and drift mobility ( $\mu$ ) can be expressed in terins of $\tau$ and $f$. The hole current density with a field in the x-direction is

$$
J_{x}=q \int_{-\infty}^{\infty} f v_{x} d \vec{v}
$$

From Eqs. (IV.1) and (IV.5)

$$
\begin{aligned}
f & =f_{0}-\tau\left(v_{x} \frac{\partial f}{\partial x}+a_{x} \frac{\partial f}{\partial v_{x}}\right) \\
& \simeq f_{o}-\tau\left(v_{x} \frac{\partial f_{0}}{\partial x}+a_{x} \frac{\partial f_{0}}{\partial v_{x}}\right)
\end{aligned}
$$

Then

$$
\left.J_{x}=q\left\{\int 0 f_{x} \vec{v} d v-\int \tau\left[v_{x} \frac{\partial f_{o}}{\partial x}+a_{x} \frac{\partial f_{o}}{\partial v}\right] x\right] x d \vec{v}\right\}
$$

The first integral vanishes because of the odd integrand. In the absence of temperature gradients the second term also vanishes. A non-degenerate distribution can be approximated by the Boltzman distribution

$$
f_{0}=n\left(\frac{m_{D}^{*}}{2 \pi k T}\right)^{3 / 2} e^{-m_{b}^{*} v^{2} / 2 k T}
$$

where $\mathbf{n}$ is the number of free charge carriers, and $\mathrm{m}_{\mathrm{D}}^{*}$ is the density of state effective mass.

Then

$$
a_{x} \frac{\partial f_{o}}{\partial v_{x}}=\frac{q_{\mathscr{E}_{X}}}{k_{B} T} v_{x} F_{0}
$$


and

$$
J_{x}=\frac{n q^{2} \mathscr{E}_{x}}{k_{B} T}\left\langle\tau v_{x}^{2}\right\rangle
$$

where $\left\langle\tau v_{x}^{2}\right\rangle \equiv \frac{\int \tau v_{x}^{2} f_{0} d \vec{v}}{\int_{f_{0}} d \vec{v}}$

The drift mobility is given by

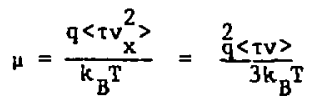

since $v_{x}^{2}=\frac{1}{3} v^{2}$. Carrying out the necessary integrations result in

$$
\mu=c_{I} T^{3 / 2}
$$

$$
\text { where } \begin{aligned}
C_{I} & =\frac{2^{7 / 2} k_{B}^{3 / 2} \varepsilon^{2}}{\pi^{3 / 2} x^{1 / 2} N_{I} z^{3} q^{3} \ln (B+1)} \\
& =\frac{3.3 \times 10^{15}}{N_{I} z^{2} \ln (B+1)}\left(\frac{m_{0}}{m^{*}}\right)^{1 / 2} \varepsilon^{2} \quad \text { in c.g.s. units }
\end{aligned}
$$

$m_{0}$ is the electrin rest mass,

and $N_{I}$ is the impurity concentration. 
APPENDIX V: Polar Optical Phonon Scattering and the Solution of Boltzman's Equation by Variational Methods

When a charge carrier interacts with an optical phonon, the scattering is an inelastic process involving considerable energy exchange between the carrier and the lattice. Consequently, the relaxation time approximation is no longer valid. A more general method must be applied to solve the Boltzman equation. A procedure based on the variational principle have been applied to metals $[143,144,145]$ and semiconductors. [146] The outline of the procedure presented here is based on the excellent review by $z$ iman. [147]

Any non-equilibrium distribution function, $f(\vec{k})$ be expanded about the equilibrium function, $f_{0}$,

$$
f(k)=f_{0}-(\vec{k}) \frac{\partial f_{0}}{\partial E} \text {. }
$$

where may be regarded as the excess energy of the distribution as a result of the transport process.

The particular form of the Fermi function allows

$$
-\frac{\partial F_{o}}{\partial E}=\frac{E_{0}\left(1-F_{0}\right)}{k_{B} T}
$$

Using the above two relationships the Boltzman transport Eq. (IV.1) can be rewritten in canonical form

$$
-\vec{v} \cdot \nabla_{r} f-\dot{\vec{k}} \nabla_{k} E=\frac{1}{k_{B} T} \int \mathscr{R}\left(\vec{k}^{2}, \vec{k}^{\prime}\right)\left\{\Phi(\vec{k})-\phi\left(\vec{k}^{\prime}\right)\right\} d \vec{k}
$$

where $\Re\left(\vec{k}^{\prime}, \vec{k}^{\prime}\right)=f_{o}\left(1-f_{0}\right) s\left(\vec{k}^{\prime} \vec{k}^{\prime}\right)$ 
This integral equation has the form

$$
\mathrm{F}(\mathrm{k})=\mathscr{Q} \Phi(\mathrm{k})
$$

in which an unknown function, $\phi$, is transformed to a known function, F, by a inear operator $\mathscr{L}$. If the inner product of two functions, $\emptyset$ and $\Psi$, can be defined by

$$
\langle\phi, \Psi\rangle \equiv \int \phi(k) \Psi(k) d k
$$

then two other properties of $\mathscr{L}$ can easily be demonstrated.

Due to the symmetry of $s$ with respect to $\vec{k}$ ar: $\vec{k} \cdot$,

$$
\langle\Phi, \mathscr{Q} \Psi\rangle=\langle\Psi, \mathscr{Q} \Phi\rangle,
$$

i.e., $\mathscr{L}$ is also symmetric or self-adjoint;

and $\langle\Phi, \mathscr{H} \phi\rangle \geq 0$

The solution of (V.5) can be obtained by evoking the variational principle which states that of all functions $\Phi$ satisfying

$$
\langle\Phi, F\rangle=\langle\Phi, \mathscr{Q} \phi\rangle
$$

the true solution gives $\langle\Phi, \mathscr{Q} \phi\rangle$ its maximum value.

An equivalent statement is that

$$
\frac{\langle\Phi, \mathscr{Q} \Phi\rangle}{\left\{\langle\Phi, F\rangle^{2}\right\}} \text { is minimized }
$$

when is the true solution.

A solution can be obtained by constructing a trial function

$$
\phi=\sum_{i} i_{i} \phi
$$

then applying the variational principle to determine the correct set of $c_{i}$ 's. 
The physical meaning of the above formalism and consequently the computation of physical parameter is demonstrated by statistical mechanics. The entropy of a system of fermious is given by

$$
S=-k_{B} \int\{f \ln f+(1-\epsilon) \ln (1-f)\} d \vec{k}
$$

The change in entropy with time to first order is

$$
\dot{\mathrm{S}} \approx-\frac{1}{\mathrm{~T}} \int \dot{\dot{\mathrm{f}}} \mathrm{d} \overrightarrow{\mathrm{k}}
$$

The original Boltzman transport equation which is a statement on the balance of changes in distribution can be reworded to one on the balance of entropy change. The contribution due to collisions is given by

$$
\begin{aligned}
\dot{S}_{c} & =\frac{1}{2 k_{B} T^{2}} \iint\left[\Phi(\vec{k})-\Phi\left(\vec{k}^{\prime}\right)\right]^{2} \mathscr{R} d \vec{k}^{\prime} d \vec{k} \\
& =\frac{1}{T}\langle\Phi, \mathscr{L} \Phi\rangle
\end{aligned}
$$

In the absence of a temperature gradient and if the external force is due to an applied field, $\mathscr{E}$, the contribution of the left side of (IV.1) is

$$
\begin{aligned}
\dot{\mathrm{S}}_{\mathrm{field}} & =-\frac{1}{\mathrm{~T}} \int \Phi(\overrightarrow{\mathrm{k}}) \overrightarrow{\mathrm{v}} \cdot \mathrm{q} \frac{\partial \mathrm{F}}{\partial \mathrm{E}} \vec{E} \mathrm{~d} \overrightarrow{\mathrm{k}} \\
& =\frac{1}{\mathrm{~T}}\langle\phi, F\rangle
\end{aligned}
$$

Eq. (V.15a) can be expressed in terms of observable parameters

$$
\dot{s}_{\text {Field }}=-\frac{1}{T} \vec{E} \cdot \vec{J}=-\frac{\rho J^{2}}{T}
$$

where $\rho$ is the resistivity, and $J$ is the current deasity. 
Eqqs. (V.14b), (V.15b), and (v.16) can be combined to give

$$
\rho=\frac{\langle\Phi, \mathscr{L} \varphi}{\{\langle\phi, F(\mathscr{E}=1)\rangle\}^{2}}
$$

where $F(\mathscr{E}=1)$ is the left side of the Boltzman equation with a unit field.

So, the variational principle can be interpreted to say that the resistivity is minimized when is the solution of the transport equation.

If the solution can be expanded in the form of Eq. ( $V .11$ ), then

$$
\rho=\frac{1}{\langle\varphi, F(1)\rangle}=\frac{1}{\sum c_{i} F_{i}}
$$

where $F_{i}=\Sigma\left\langle\phi_{i} \mathscr{S}_{j}\right\rangle c_{j}$ follows from (v.5).

The coefficients $c_{n}$ can be computed by inverting (v.5)

$$
\rho=\frac{1}{\sum_{i j} F_{i}\left(\mathscr{Q}^{-1}\right)_{i j} F_{j}}
$$

where $\left(\mathscr{C}^{-1}\right)_{i j}$ is an element of the inverse of the operator matrix.

Mobility can then be defined by

$$
\mu \times \frac{j}{n q} \sum_{i j} F_{i}\left(\mathscr{S}^{-1}\right) i j_{j} F_{j}
$$

The elements $F_{i}$ with a unit field is just the electric current density due to changes in the trial distribution function

$$
F_{i}(\&=1)=J_{i}=\int q v_{k} \phi_{i} \frac{\partial F_{o}}{\partial E} d \vec{k}
$$


The elements of the transformation matrix are

$$
\begin{aligned}
\mathscr{L}_{\mathrm{i} j} & \equiv{ }_{\mathrm{i}}, \mathscr{L}_{\left.\phi_{j}\right\rangle} \\
& =\frac{1}{k_{\mathrm{B}} \mathrm{T}} \iint\left[\Phi_{\mathrm{i}}(\vec{k})-\phi_{\mathrm{i}}\left(\vec{k}^{\prime}\right)\right] \mathscr{R}\left[\phi_{j}(\vec{k})-\phi_{j}\left(\vec{k}^{\prime}\right)\right] \mathrm{d} \vec{k}^{\prime} \mathrm{d} \vec{k}
\end{aligned}
$$

The inverse is then obtained by the usual matrix formulas.

The interaction Hamiltonian for scatcering of an electron or hole by a polar lattice involving the absorption of an opeical phonon is [148]

$$
\left\langle\underset{\vec{k}}{\psi_{\vec{k}}}, n_{\vec{k}} 1 \mathrm{H}^{\prime}\right| \mathrm{n}_{\vec{k}}-1, \psi_{\vec{k}},>=-4 \pi i\left(\frac{q^{2} \hbar}{2 \gamma \omega v}\right)^{1 / 2} \frac{n_{k}}{k}
$$

where $\frac{1}{\gamma}=\frac{\omega^{2}}{4 \pi}\left(\frac{1}{\varepsilon_{\infty}}-\frac{1}{\varepsilon}\right)$

$$
\omega=\left(\varepsilon / \varepsilon_{\infty}\right)^{1 / 2} \omega_{\Gamma}
$$

$E_{\infty}$ is the dielectric constant in high frequency limit

${ }^{\omega_{r}}$ is the Reststrahlen frequency

$V$ is the volume which is a normalization factor that disappears on integration over real space.

Then

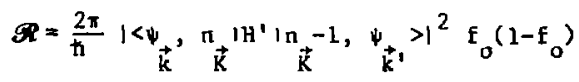

The case of pinonon emission has not been considered because in the temperature range of interest $\left(T \leq 300^{\circ} \mathrm{K}\right)$ very few electrons have sufficient energy to allow for the creation of an optical phonon. 
The basis of the trial function can have the form

$$
\phi_{i}(\vec{k})=(E)^{i} \vec{k} \cdot \hat{u}
$$

where $\hat{\mathbf{u}}$ is a unit vector in the direction of the electric field

Eqs. (V.20) through (V.26) can then be used to compute 4 to any desired degree of accuracy. With just one term in the expansion (V.11)

$$
\mu=\frac{3}{\pi^{1 / 2} 2^{7 / 2}} \frac{\gamma(k T)^{3 / 2}}{\mathrm{qm}^{*^{3 / 2}}} \frac{\left(\mathrm{e}^{\mathrm{z}}-1\right)}{\mathrm{e}^{\mathrm{z} / 2} \mathrm{~K}_{1}\left(\frac{1}{2} \mathrm{z}\right)}
$$

where $z=\frac{\omega}{k_{B} T}$

and $K_{1}(z / 2)$ is a modified Bessel function.

This approximation is most accurate at high temperatures where $z$ is small. In the limit of small z

$$
K_{1}\left(\frac{1}{2} z\right)=\frac{2}{z}
$$

and $\quad \mu_{\text {H.T. }}=\frac{3 \gamma h \omega\left(k_{B} T\right)^{1 / 2}\left(e^{2}-1\right)}{\pi^{1 / 2} 2^{9 / 2} q m^{k^{3 / 2}}}$

$$
\simeq \frac{3 \gamma(\hbar \omega)^{2}}{\pi^{1 / 2} 2^{9 / 2} q \pi^{3 / 2}\left(k_{B} T\right)^{1 / 2}}
$$

At Low temperatures an additional term in the expansion ( $V . I I$ ) gives

$$
\mu_{\text {L.T. }}=\frac{r(\hbar \omega)^{3 / 2}\left(e^{2}-1\right)}{\pi 2^{5 / 2} \mathrm{qm}^{*^{3 / 2}}}
$$


This derivation was originally due to Howarth and Sondheimer [146] using variational techniques. Mott and Frohlich [149] and Frohlich, et al ${ }^{[150]}$ obtain similar expressions with the same temperature dependence with a relaxation time approximation. 


\section{Keferences}

1. Reynolds, D. C., Leies, G., Antes, L. L. and Marburger, R. E., Phys. Rev. 96, 533 (1954).

2. Reynolds, D. C. and Czyzak, S. J., Phys. Rev. 96, 1705 (1954).

3. Williams, R. and Bube, R. H., J. Appl. Phys, 31, 968 (1960).

4. Grimneiss H. G. and Memming R., J. Appl. Phys. 33(7), 2217 (1962).

5. Cusano, D. A., Solid-State Electron, 6, 217 (1963).

6. Woods, J. and Champion, J. A., J. Electron Control 2, 243 (1950).

7. Fabricius, E. D., J. Appl. Phys. 33, 1597 (1962).

8. Hill, E. R. and Keramidas, B. G., Rev. Phys. Appl. $1(3), 189$ (1966).

9. Selle, B., Ludwig, W. and Mach, R., Phys. Stat. Sol. 24, K149 (1967).

10. Shiozawa, L. R., Sullivan, G. A., Augustine, F., Conf. Rec. 7 th IEEE Photovoltaic Specialist Conf., 1968, p. 39.

11. Shiozawa, L. R., Augustine, E., Sullivan, G. A., Smith, J. M., III, and Cook, W. R., Jr., "Research on the Mechanisms of the Photovolcaic Effect in High Efficiency CdS Thin-Film Solar Cells," Final Report, Contract AF33(615)-5224 (1969).

12. Nakayama, N., Japan J. Appl. Phys. $\underline{8}(4), 450$ (1969).

13. Naikayama, N., Japan. J. Appl. Phys. 10(10), 1450 (1971).

14. Palz, W., Besson, J., Nguyen Duy, T. and Vedel J., Conf. Rec., 9th IEEE Photovoltaic Specialists Conf., 1972, p.91.

15. re Velde, T. S. and Pieleman, J., Philips Res. Repts. 28, 573 (1973).

16. Cook, W. R., Jr., Shiozawa, L. and Augustine, F., J. Appl. Phys. 41 (7), $3058(1970)$.

17. Buerger, M. J. and Buerger, N. W., Am. Mineral 29, 55 (1944). 
13. Djurl, S., Acta. Chem. Scan. 12, 415 (1958).

19. Cook, W. R., Ph. D. Thesis, "The Copper-Sulfide Phase Diagram," 1977.

20. Rosedoom, E. H., Jt., Econ. Geol. 61, 64I (1966).

21. Taked., H., Donnay, J. D. H., Roseboom, E. H. and Appleman, D. E., Leit. KI t. 125,404 (1967).

22. Elisiev, E. N., Rudenko, L. E., Sinev, L. A., Koshurnikov, B. K. and Solovov, N. I., Mineralog. Sb. L'vovsk, Gos. Univ. 18, 365 (1964).

23. Mulder, B. J., Phys. Stat. Sol. (a) 13,79 (1972).

24. Buerger, N. W., Econ. Geol. 36, 19 (1941).

25. Sorokin, G. P., Andronik, I Ya and Kovtun, E. V., Izv. Akad, Nauk. SSSR, Neorg. Mat. 11(12), 2129 (1975).

26. Armantrout, G. A., Miller, D. E., Vindelov, K. E. and Brown, I. G., J. Vac. Sci. Technol. 16(2), 212 (1979).

27. Eisenmann, L., Ann. Phys. (Leipzig)(6) 10, 129 (1952).

28. Marshall, R. and Mitra, S. S., 36(12), 3882 (1965).

29. Mulder, B. J., Phys. Stat. Sol. (a) 18, 633 (1973).

30. Mulder, B. J., Phys. Stat. Sol. (a) 15, 409 (1973).

31. Sorokin, G. P., Papshev, Yu. M. and Oush, P. T., Fiz. Tver. Tela, I (7), 2244 (1965).

32. Nakayama, N., J. Phys. Soc. Jap. 25, 290 (1968).

33. Ramoin, M., Sorbier, J. P., Bretzner, J. F. and Martinuzzi, S., C. R. Acad. Sci. 268,1097 (1969).

34. Couve, S., Gouskov, L., Szepessy, L., Vedel, J. and Castel, E., Thin Solid Films, 15, 223 (1973).

35. Lofershi, J., J. Appl. Phys. 27(7), 777 (1956)

36. Burstein, E., Phys. Kev. 93, 632 (1954). 
37. Moss, T. S., Proc Phys. Soc. (London) B76, 775 (1954).

38. Snewchun, J., Loferski, J. J., Wold, A., Arnott, R., De Meo, E. A., Beauliev, R., Hu, C. C. and Harvey, H. L., Conf. Rec., Ilth IEEE Photovoltaic Specialists Conf., 1975, p.482.

39. Dash, H. C. and Newman, R., Phys. Rev., 99(4), 1151 (1955).

40. Te Velde, T. S., Energy Conv. 14(3/4), 111 (1975).

41. Loferski, J. J., Shewchun, J., De Heo, E. A., Arnott, R.,: Crisman, E. E., Beaulieu, R., Hwang, H. L. and Wu, C. C., Conf. Rec., 12th IEEE Photovoltaic Specialist Conf., 1976, p.496.

42. Loferski, J. J., Shewciun, J., Mittleman, S. D., De Meo, E. A., Arnott, R., Hwang, it. L.: Beaulieu, R. and Chapman, G., Solar Energy Mat. $1(1,2), 157$ (1979).

43. Yee, J. H., Photovoltaics Advanced Materials Review Meeting, 1978, p.61.

44. Hirahara, E., J. Phys: Soc. Japan 6(6), 428 (i951).

45. Hirahara, E., J. Phys. Soc. Japan 6(6), 422 (1951).

46. Hirahara, E., J. Phys. Soc. Japan 2, 211 (1947).

47. Miyatani, S. and Suzuki, Y., J. Phys. Soc. Japan $\underline{8}, 680$ (1953).

48. Miyatani, S., J. Phys. Soc. Japan 11(10), 1059 (1956).

49. Okamoto, K. and Kawai, S., Japan J. Appl. Phys. 12(8), 1130 (1972).

50. Yokota, I., J. Phys. Soc., Japan $\underline{8}(5), 595$ (1953).

51. Abdullaev, G. B., Aliyarova, Z. A., Zamanova, E. H. and Asadov, G. A., Phys. Stat. Sol. 26, 65 (1968).

32. Bougnot, J., Guastavino, F., luquet, H. and Sodini, D., Phys. Stat. Sol. (a)8, K93 (1971).

53. Sorokin, G. r. and Pararieniko, A. P., Izv. Vuz. Fig. (5), 91 (1966).

54. Sorokín, G. P., Andronik, I. Ya and Kovtum, E. V., Izv. Akad. Nauk. SSSR., Neorg. Mat. $\underline{11}(12), 2129$ (1975). 
55. Thompson, A. G., Cardona, M., Shaklee, K. L., and Wooley, J. C., Phys. Rev. 146, 601 (1966).

56. Bardeein, J., Blatt, F. J., and Hall, L. H., Photoconductivity Conference, J. Wiley, New York, 1956, P. 146.

57. Avery, D. G., Goodwin, D. W., Lawson, W, D., and Moss, T. S., Proc. Phys. Soc. B 67, 761 (1954).

58. Tanenbaum, M. and Briggs, H. B., Phys. Rev. 91, 156 (1953).

59. Herman, F., J. Electron. 1, 103 (1955).

60. J. I. Pankove and Aigrain, P., Phys. Rev. 126 (3), 956 (1961).

61. Haas, C., Phys. Rev. 125, 1965 (1962).

62. Urbach, F., Phys. Rev. 92, 1324 (1953).

63. Pankove, J. I., Phys. Rev. 140 (6A), :2059 (1965).

64. Piper, W. W., Phys. Rev. 92, 23 (1953).

65. Keller, S. P. and Pettit, G. D., Prague Semiconductor Conference (1960), p. 39 .

66. Baltensperger, W., Phil. Mag. 44, 1355 (1953).

67. Lax, M., and Phillips, J. C., Phys. Rev, 110, 41 (1958).

68. Redfield, D., Phys. Rev. 130, 916 (1963).

69. Franz, W., Z. Natur forsch. 13a, 484 (1958).

70. Keldysh, L. V., Sov. Phys. JETP ㄱ, 788 (1958).

71. Pankove, J. I., Optical Processes in Semiconductors, Dover, New York, 1971 , P. 47 .

72. Wang, S., Solid State Electronics, McGraw-Hill, New York, 1966, p. 369 .

73. Kane, E. O., J. Phys. Chem. Solids 12, 181 (1959).

74. Dow, J. D, and Kedfield, D., Phys. Rev. Lett. 26 (13), 762 (1971).

75. Dow, J. D., Phys. Rev. B 4 (8), 3358 (1970). 
76. Duke, C. B. and Alferieff, Phys. Rev. 145, 583 (1960).

77. Blatt, F. J., Physics of Electronic Conduction in Solids, McGraw-Hil1, New York, 1968, p. 338.

78. Ashcroft, N. W. and Mermin, N. D., Solid State Physics, Holt, Rinehart and Winston, New York, 1976, p. 53.

79. Fan, H. Y., Spitzer, W. G., and Collins, R. J., Phys. Rev. 101, 566 (1956).

80. Visvanathan, S., Phys. Rev. 120,376 (1960).

B1. Moglich, F. and Rompe, R., 2. Physik L19, 472 (1942).

82. Bardeen, J. and Shockley, W., Phys. Rev. 80, 72 (1950).

83. Fan, H. Y., Phys. Rev 82 (6), 898 (1951).

84. Varshi-i, Y. P., Physica 34,149 (1967).

85. Spr: $\perp 1$, R., Modern Physics, John Wiley, New York, 1963.

86. Asnin, V. M. and Rogatcheu, A. A., Sov. Phys.--Solid State $\underline{\text {, }}$ 1730 (1963).

87. Holonysk, N., Johnson, M. R., and Rossi, J. A., Appl. Phys, Lett. 12, 151 (1968).

88. Redfield, D. and Afromowitz, M. A., Appl. Phys. Lett. 11,138 (1967).

89. Bardeen, J. and Schockley, W., Phys. Rev. 80 (1), 72 (1950).

90. Wang, S., op. cit., p. 220.

91. Hutson, A. R., Phys. Rev. Lett. $\underline{4}$ (10), 505 (1960).

92. Devlin, S. S., Appendix 6 L. R. Shiozawa and J. M. Jost, 1965, Research on II-VI Compound Semiconductors, Final Technical Report, Contract \#AF33(657)-7399, U.S.A.F. Aeronautical Research Laboratories, Wright Patterson Air Force Base, Ohio.

93. Harrison, W. A., Phys. Rev. 101, 903 (1956).

94. Meijer, H. J. G. and Polder, D., Physica XIX, 255 (1953). 
95. Kroger, F. A., Vink, H. J., and Volger, J., Philips Res. Rep. 10, 39 (1955).

96. Howarth, D. J. and Sondheimer, E. H., Proc. R. Soc. Land. Ser. A $219,53(1953)$.

97. Ehrenreich, H., J. Phys. Chem. Solids 8, 130 (1959).

98. Frohlich, H. and ifott, N. F., Proc. Roy, Soc A, 171, 496 (1939).

99. Harrison, J. W. and Hauser, J. R., J. Appl. Phys. 47 (1), 292 (1976).

100. Matthiessen, A., Rep. Brit. Ass. 32, 144 (1862).

101. Ziman, J. M., Electrons and Phonons, Oxford University Press, London, $1^{a r} 3$, P. 434 .

102. Brooks, f., Phys. Rev. 83, 879 (1951).

103. Dingle, R. B., Phil. Mag. 46, 831 (1955).

104. Erginsoy, C., Phys. Rev. 79, 1013 (1950).

105. Kamins, T. I., J. Appl. Phys. 42 (11), 4357 (1971).

106. Petritz, R. L., Phys. Rev. 104, 1508 (1956).

107. Many, A., Goldstein, Y., and Grover, N. B., Semiconductor

Surfaces, North-Holland, New York, 1965, p. 309.

108. Bube, R., "Electronic Transport in Polycrystalline Films," in Annual Review of Material Science, Vol. 5, 1975, p. 201.

109. Anderson, J. C., Advan. Phys. 19, 311 (1970).

110. Hil1, E. R. and Kerimidas, B. G., IEEE Trans. Electron Devices ED-14, 22 (1967).

111. Armantrout, G. A., Yee, J. H., Fischer-Colbrie, E., Miller, D., Hsieh, E., Leong, J., Vindelov, K. E. and Brown, T. G., Conf. Rec., 13th Photovoltaic Specialist Conf., 1978, p.383. 
112. Sources of general information for preparing the discussion on sputtering were:

Maissel, I. I. in Physics of Thin Films, Vol. 3, Haas, G. and Thun, R. E. (eds.), Academic Press, New York, 1966, P.61.

Wehner, G. K. and Anderson, G. S., in Handbook of Thin Films Technology, HcGraw-Hill, New York, 1970, P.3-1.

Maissel, L., in Handbook of Thin Films Technology, McGraw-Hill, New York, 1970, p.4-1.

Discussions with Dr. L. Lamont and Messrs. D. Miller and K. Vindelov are gratefully acknowledged.

113. Pease, R. S., Rend. Scuola. Intern. Fis. Enrico Fermi 1959, 138.

114. Stuart, R. V. and Wehner, G. K., J. Appl. Phys. 33, 2345 (1962).

115. Stuart, R. V. and Wehner, G. K., J. Appl. Phys. 35, 1819 (1964).

116. Koenig, H. R. and Maissel, I. I., IBM J. Res. Develop. 14, 168 (1970).

117. The derivation of the voltage distribution follows that of Koenig and Maissel, but the results in $\mathrm{Eq} .(4-7)$ is different because current, instead of current density, was taken as the conserved quantity.

118. Heavens, 0. S., Optical Properties of Thin Films, Butterworth, London, 1955, P. 77 .

119. Denton, R. E., Campbel1, R. D. and Tomlin, S. G., J. Phys. D: Appl. Phys. 5, 852 (1972).

120. van der Pauw, L. J., Philips Res. Repts. 13, 1 (1958).

121. Beer, A. C., Galvanometric Effects in Semiconductor, Academic Press, New York, 1963, P. 215 .

122. Partain, L. D. and Lakshminarayana, M. R., J. Appl. Phys. 47 (3), $1015(1976)$.

123. Blatt, F. J., Physics of Electronic Conduction in Solids, NcGraw-Hill, New York, 1968, p. 287 .

124. Nag, B. R., Theory of Electrical Transport in Semiconductors,

Pergamon Press, Oxford, 1972, p. 117. 
125. Devlin, S. S., "Transport Properties," in Physics and Chemistry of II-VI Compounds, North-Holland, 1967, p. 551.

126. A. D. Jonath, W. W. Anderson, J. A. Thorton, and D. G. Cornog, J. Vac. Sci. Technol. 16 (2), 200 (1979).

127. J. Bougnot, F. Gustavino, G. M. Moussalli, and M. Savelli, in International Workshop on Cadium Sulfide Solar Cells and Other Heterojunction, April 30 - May 2, 1975, Newark, Delaware, edited by K. Böer and J. D. Meals in (Institute of Energy Conversion, University of Delaware, Newark, 1975), p. 337.

128. F. J. Blatt, Physics of Electronic Conduction in Solids, McGraw-Hil1, New York, 1968, p. 174.

129. L. R. Shiozowa, F. Augustine, G. A. Sullivan, J. M. Smith, III, and W. R. Cook, Jr., op. cit., p. 171 .

130. H. M. Windawi, Conf. Rec., 11th IEEE Photovoltaic Specialist Conf., 1975, p. 464 .

131. Institute of Energy Conversion, University of Delaware, Quarterly Progress Rept., E(49-18)-2538.

132. E. A. Fagen, private communication.

133. The assistance of Messrs. Barry $\mathrm{Ch}$ in and Lee Johnson in arranging for the use of the $x$-ray diffractometer at the Lawrence Berkeley Laboratory is gratefully acknowledged.

134. H. Kobayashi and C. Wagner, J. Chem. Phys. 26 (6), 1609 (1957).

135. Schiff, L. I., Quantum Mechanics, McGraw-Hill, New York (1968), p. 279 .

The c.g.s. units are used throughout this derivation.

136. Smith, R. A., Wave Mechanics of Crystalline Solids, John Wiley, New York $(1961)$, p. 409 .

137. Calloway, J., Energy Band Theory, Academic Press, New York, 1964, p. 288 .

138. Wang, S., Solid-State Electronics, McGraw-Hil1, New York, 1966, P. 670 .

139. Blatt, F. J., op. cit., P. 111. 
140. Reif, F., Fundamentals of Statistical and Thermal Physics, McGraw-Hili, New York, 1965, p. 521 .

141. Wilson, A. H., The Theory of Metals, Cambridge University Press, Cambridge, 1953, P. 8 .

142. Anderson, E. E., Modern Physics and Quantum Mechanics, W. B. Saunders Co., Philadelphia, 1971, P. 402.

143. Kohler, M., Z. Phys. 124, 772 (1948).

144. Sondheimer, E. H., Proc. Royal Society A., 203, 75 (1950).

145. Wilson, A. H., op. cit., P. 307.

146. Howarth D. J. and Sondheimer, E. H., Proc. Royal Society A, 219, 53.

147. Ziman, J. M., op. cit., p. 275.

148. Ibid., p. 211.

149. Frohlich, H. and Mott, N. F., Proc. Roy. Soc. A., 171, 496 (1939).

150. Frohlich, H., Pelzer, H., and Zienau, S., Phil. Mag. 41, 221 (1950). 